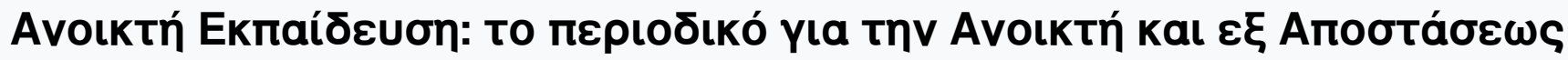

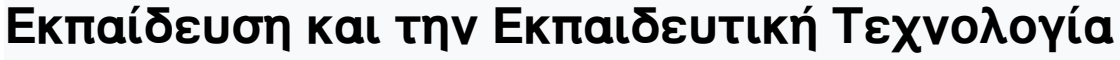

Tóp. 5, Ap. 1 (2009)

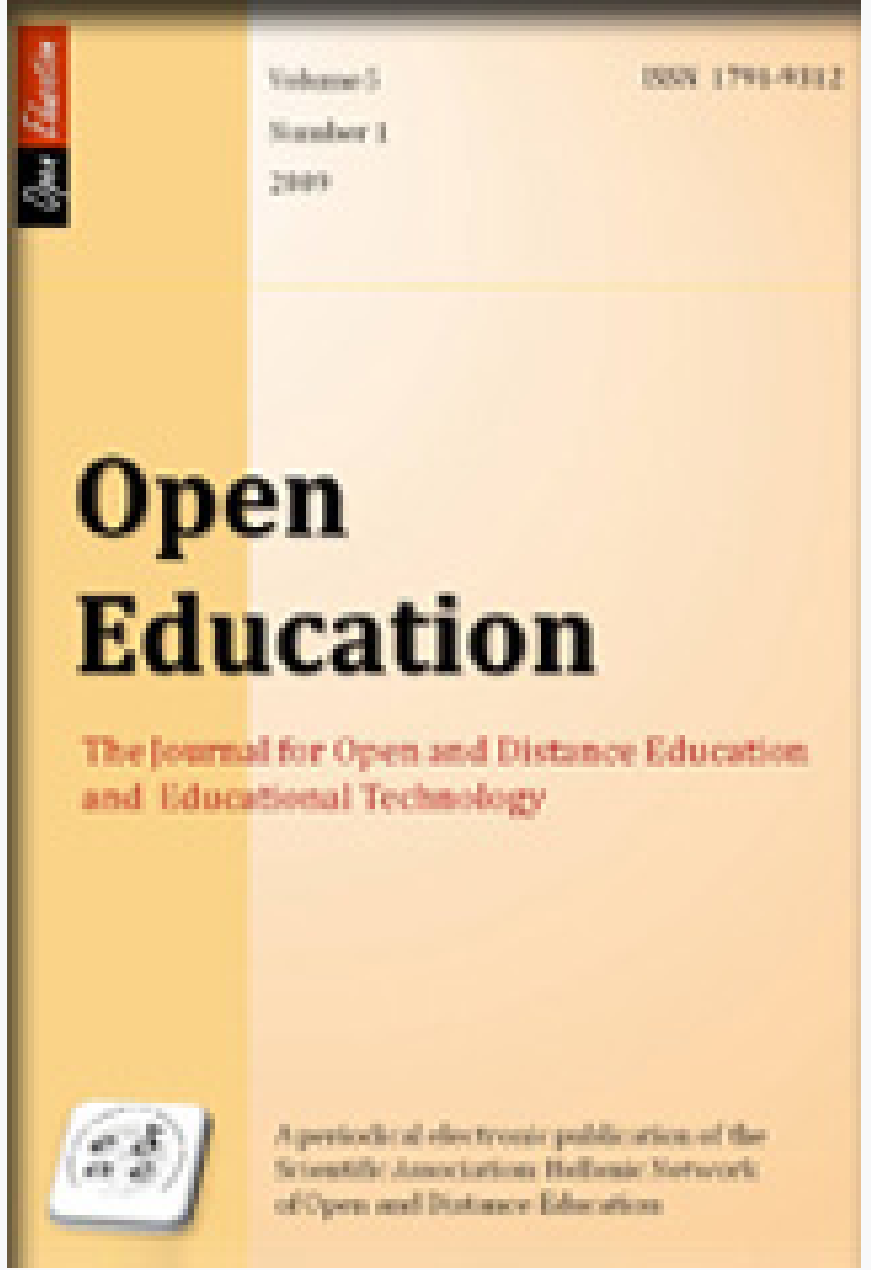

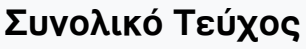

¿UVoגıKó TEúXos

doi: $10.12681 /$ jode. 9845

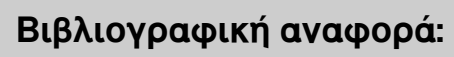




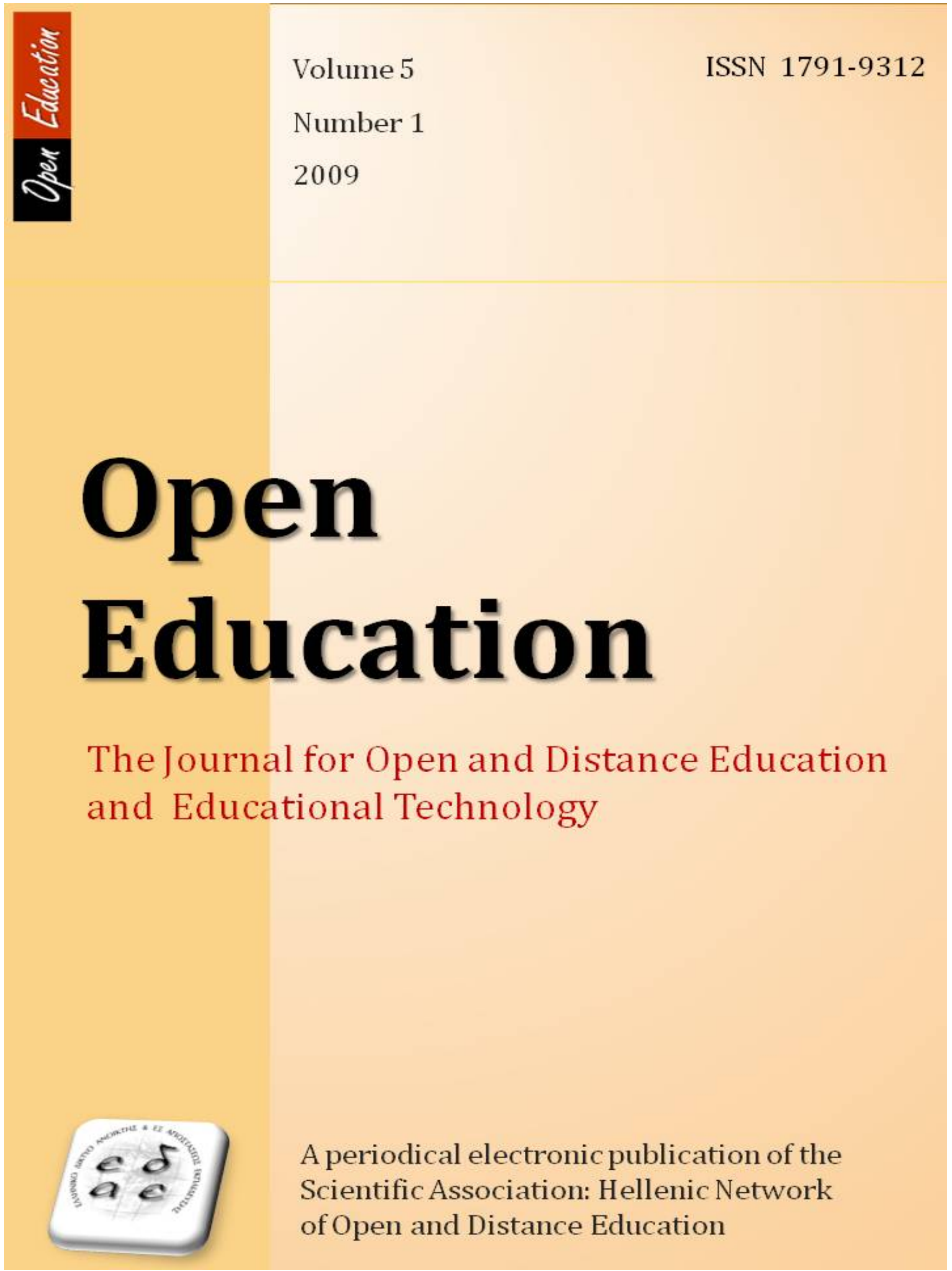




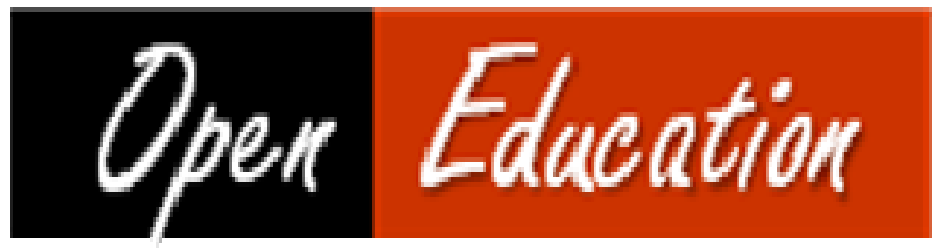

A periodical electronic publication of the Scientific Association: Hellenic Network of Open and Distance Education

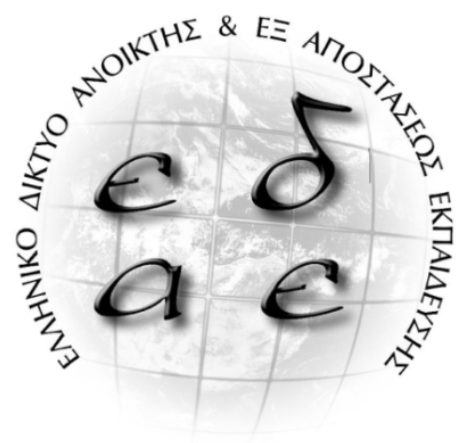

\section{Editorial Communication}

\section{Antonis Lionarakis}

Acssociate Professor

Hellenic Open Universirty \&

Hellenic Network of Open \& Distance Education

Sahtouri 23, 26222 Patra

Greece

E-mail : alionar@eap.gr

Web site : http://www.openedu.gr/

Books for review should be addressed to the above postal address

Hellenic Network of Open and Distance Education

2009 (C) OPEN EDUCATION ISSN: $1791-9312$

The responsibility of the editing of the articles lies with the authors 


\section{CONTENTS}

Editorial (In Greek)

Antonis Lionarakis

Constructivist design and evaluation of interactive educational software: a research-based approach and examples (In English)

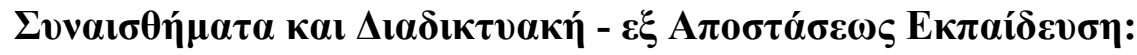

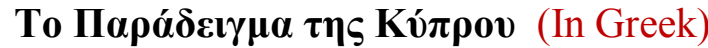

Emotions and Online-Distance Education: The Case of Cyprus

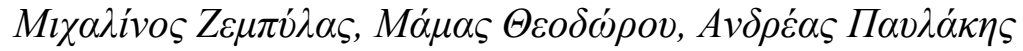

Distance Education: access for all (In English)

Anna Koulikourdi

What kind of Haptic devices and applications are needed in education? Requirements, Specifications and hands-on experience derived from an IST project (In English) S. P. Christodoulou, D. M. Garyfallidou, G. S. Ioannidis,

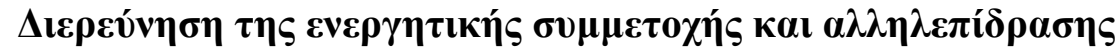

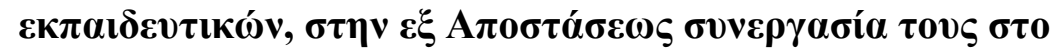

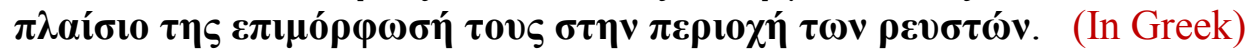
Investigation teachers' active participation and interaction, concerning distance collaboration, during their in service training in fluids.

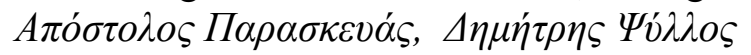

Blended Learning: The transformation of Higher Education Curriculum (In English)

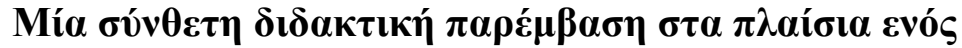

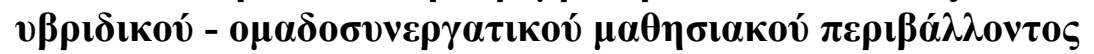

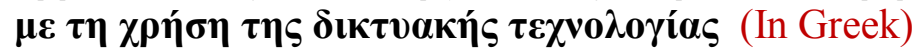
A pedagogical design of a complex instructional intervention with the creation of a hybrid collaborative learning environment

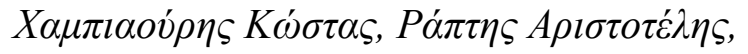




\section{Editorial}

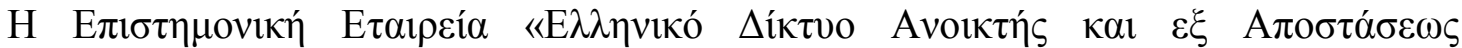

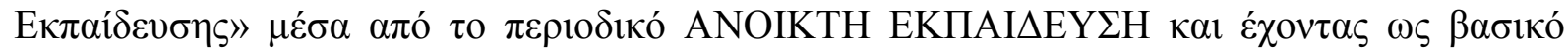

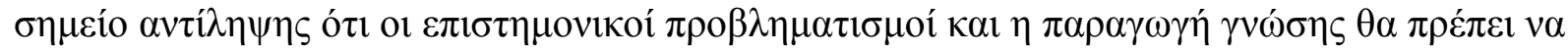

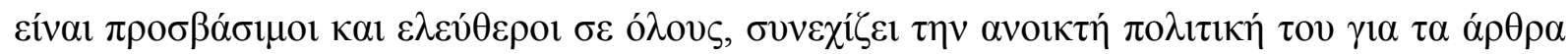

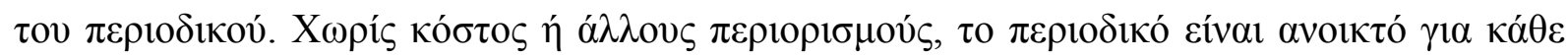

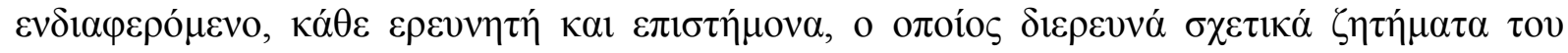

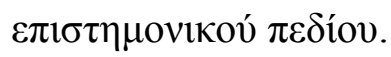

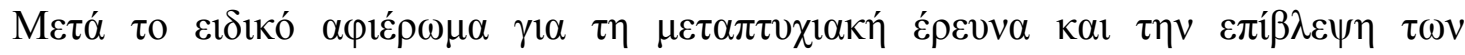

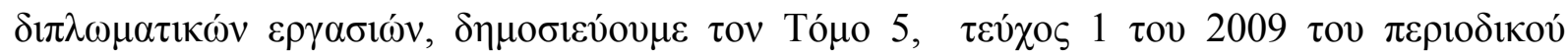

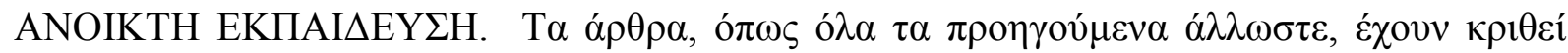

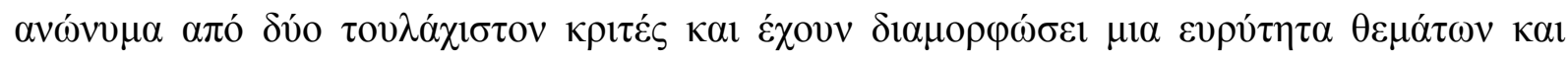

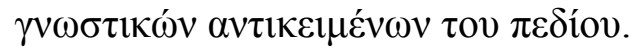

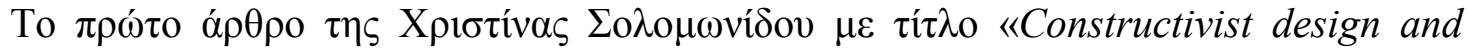
evaluation of interactive educational software: a research-based approach and

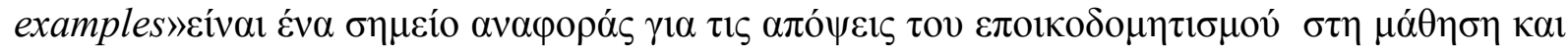

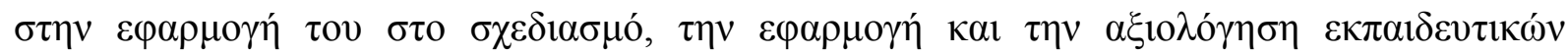

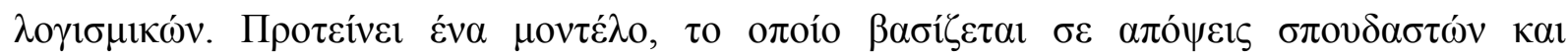

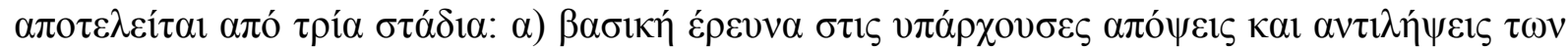

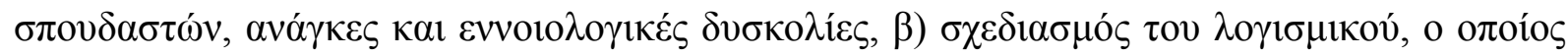

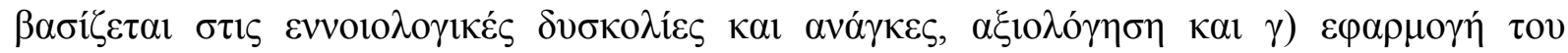

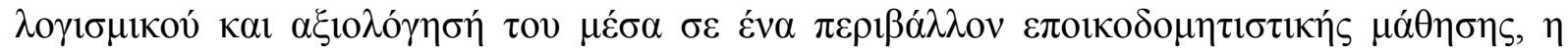

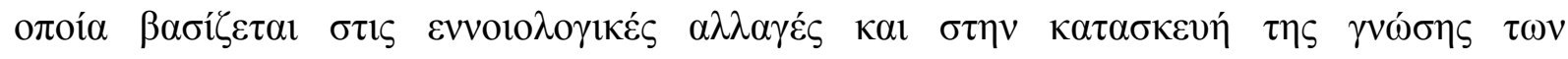
$\sigma \pi \circ v \delta \alpha \sigma \tau \omega ́ v$.

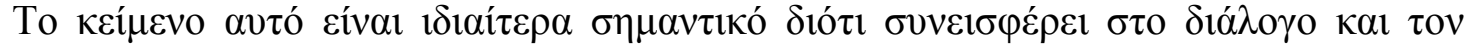

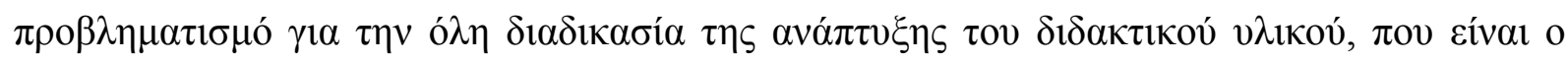

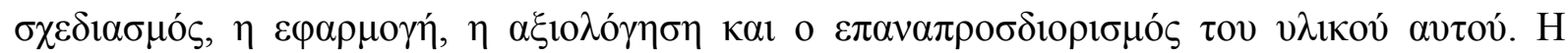

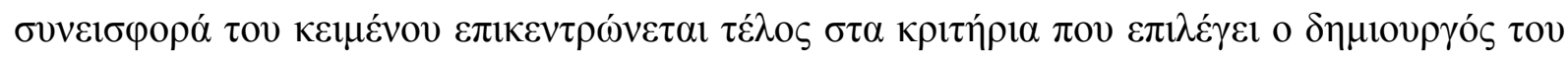
v入ıкои́.

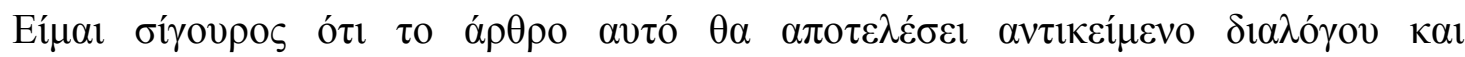

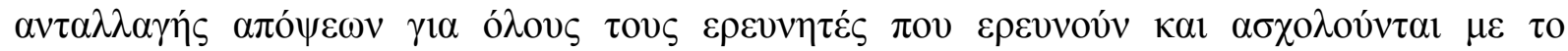

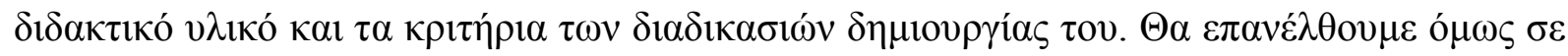

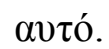

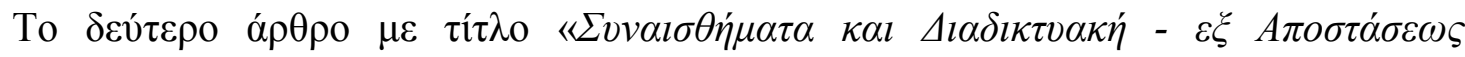

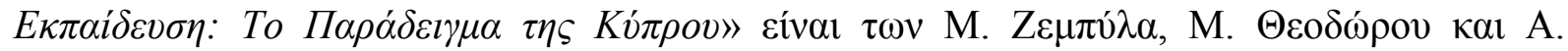

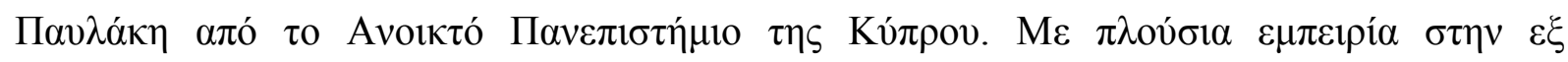

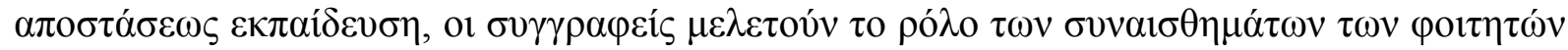

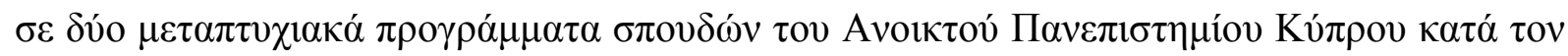




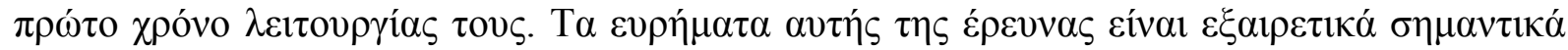

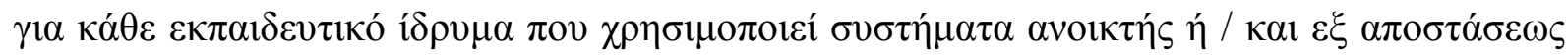

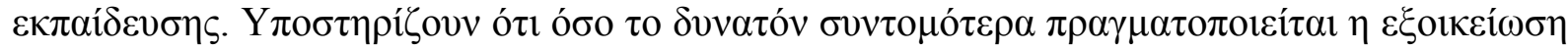

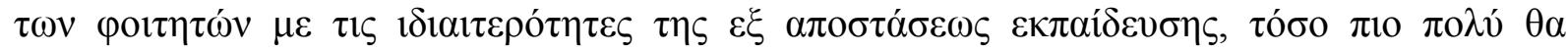

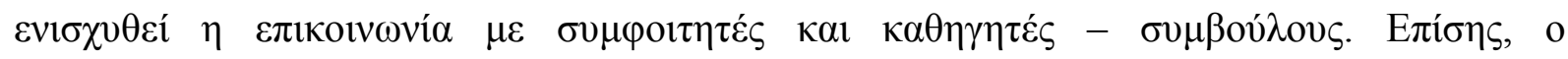

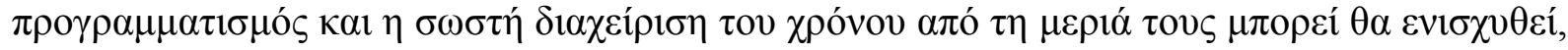

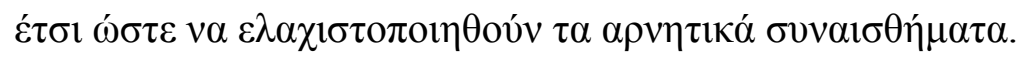

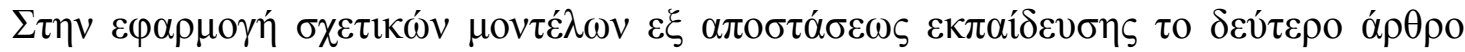

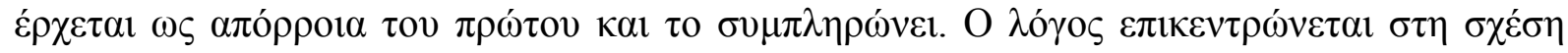

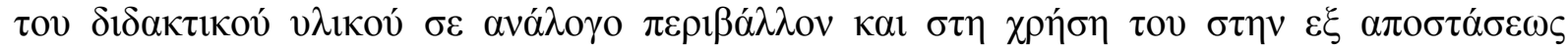

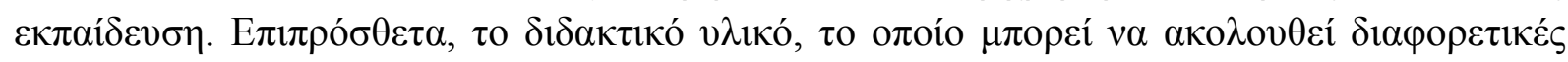

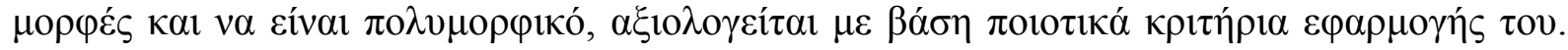

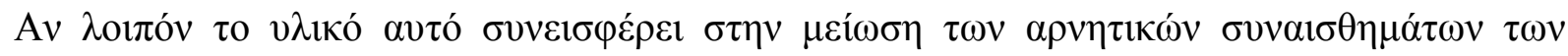

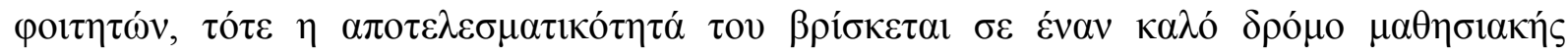
$\delta 1 \alpha \delta 1 \kappa \alpha \sigma i ́ \alpha \varsigma$.

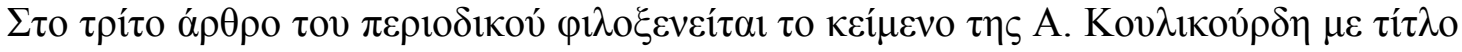

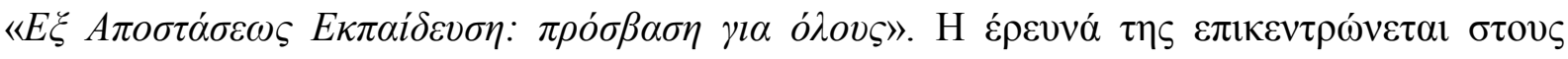

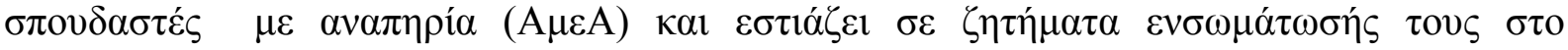

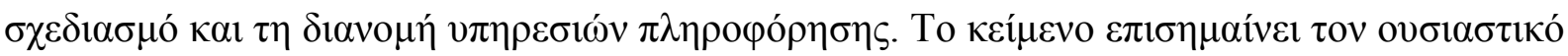

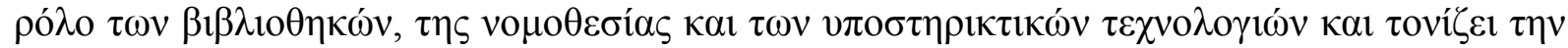

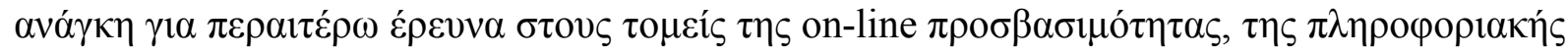

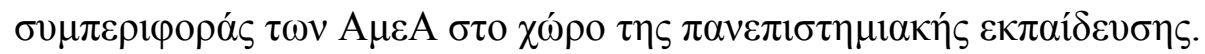

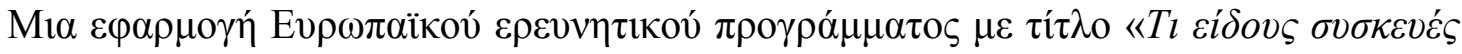

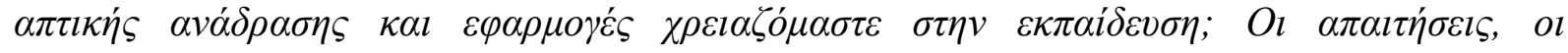

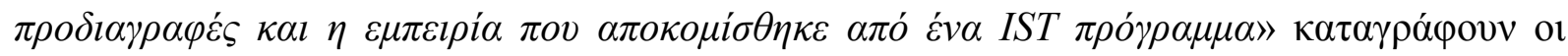

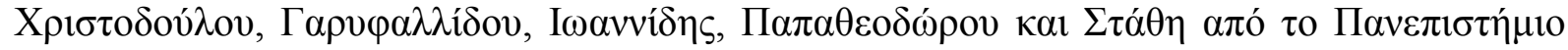

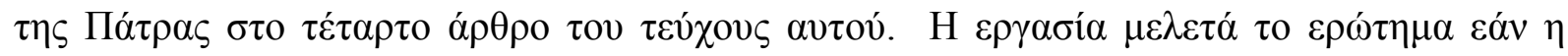

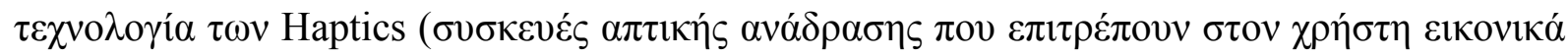

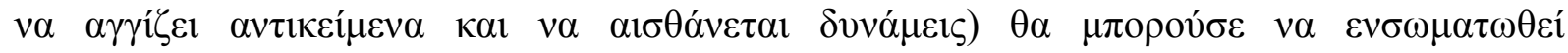

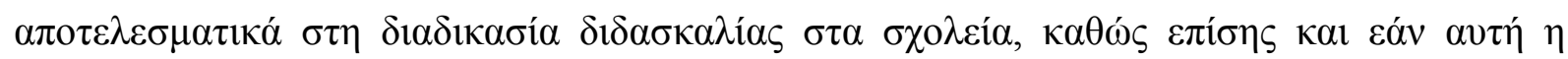

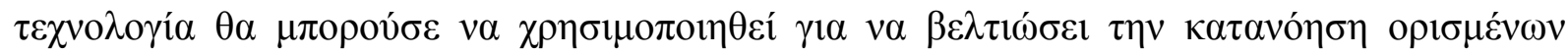

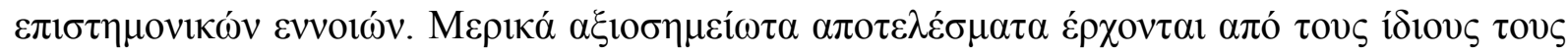

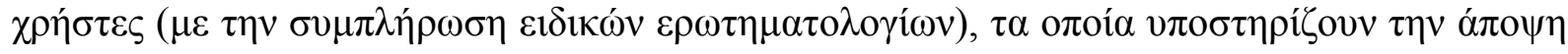

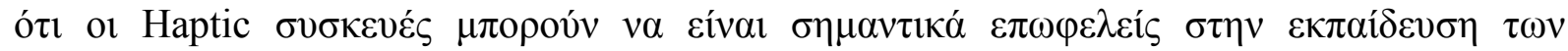
$\mu \alpha \theta \eta \tau \omega ́ v ~ \sigma \varepsilon$ ó $\lambda \varepsilon \varsigma \tau \iota \varsigma \beta \alpha \theta \mu i ́ \delta \varepsilon \varsigma$.

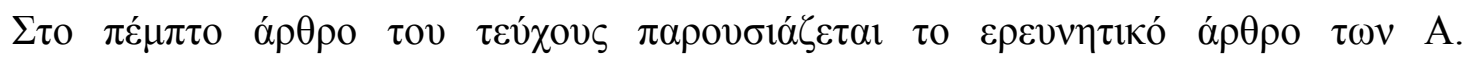

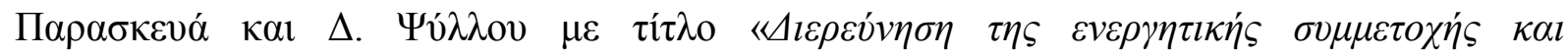

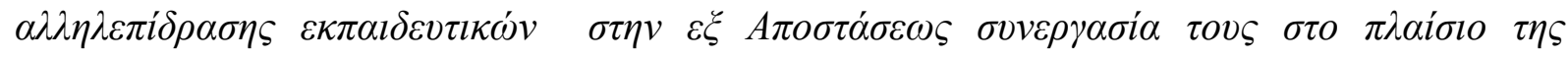

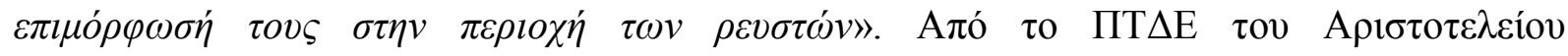

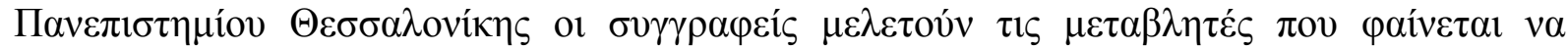




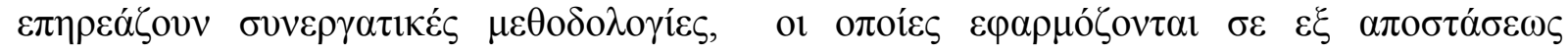

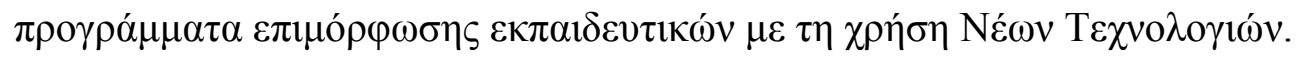

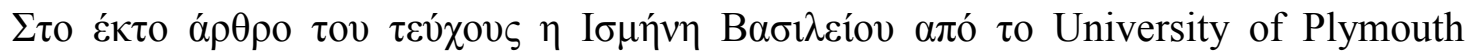

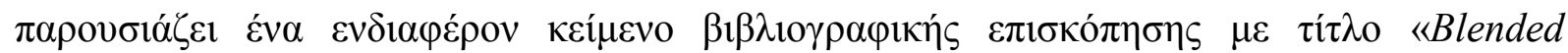

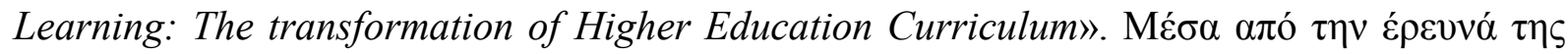

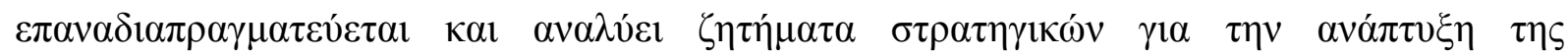

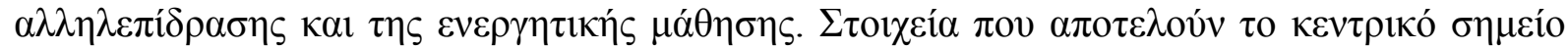

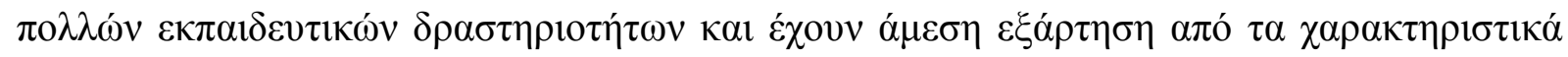

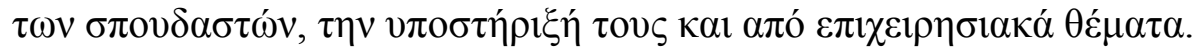

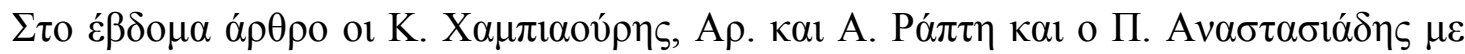

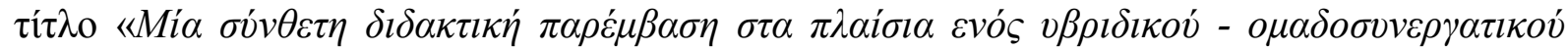

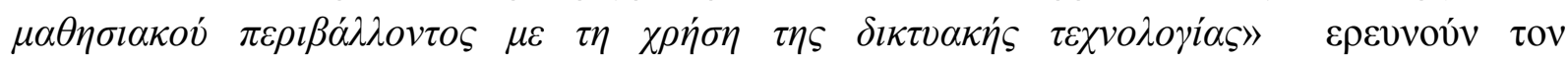

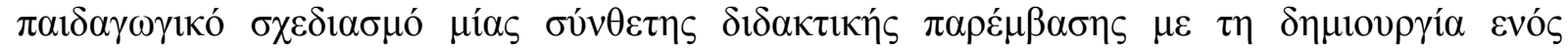

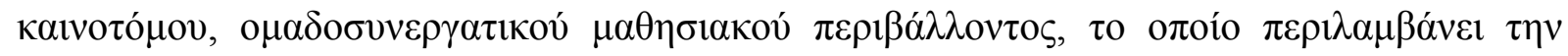
$\tau \eta \lambda \varepsilon \delta 1 \delta \alpha \sigma \kappa \alpha \lambda i ́ \alpha \kappa \alpha \imath \tau \eta \nu ~ \tau \eta \lambda \varepsilon \varepsilon \sigma v \varepsilon \rho \gamma \alpha \sigma i ́ \alpha$.

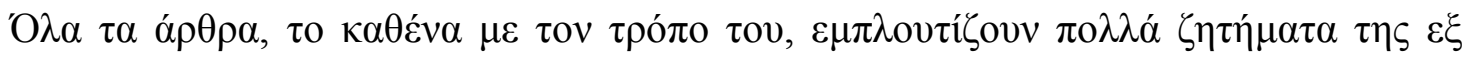

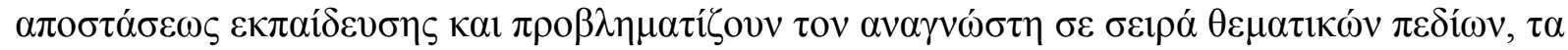

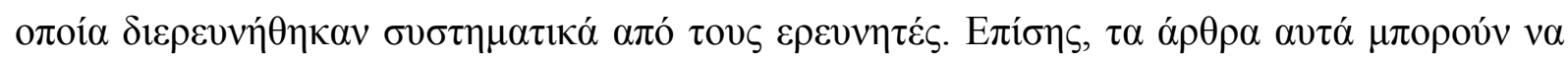

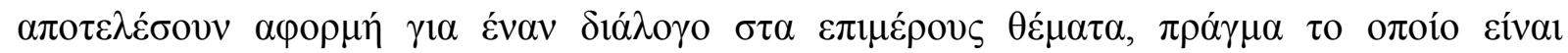

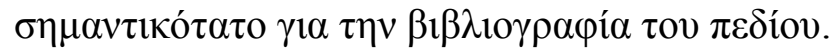

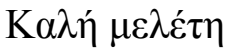




\title{
Constructivist design and evaluation of interactive educational software: a research- based approach and examples
}

Christina Solomonidou

University of Thessaly, Professor e-mail:xsolom@uth.gr

\begin{abstract}
The paper introduces constructivist views of learning as a theoretical background to inform the design, implementation and evaluation of quality interactive multimedia educational software. It reviews various constructivist views of learning and also constructivist technology-mediated learning. It proposes an approach to design and evaluation of constructivist educational software, which is based on research in students' ideas, and comprises three stages: a) initial research into students' existing ideas, conceptions, conceptual difficulties and needs, b) design of the software based on students' conceptions and conceptual needs, and formative evaluation of it, and c) software implementation and evaluation within a constructivist learning environment, based on students' conceptual change and construction of appropriate knowledge by them. This research-based approach is compared to other existing models of design educational software environments. In order to illustrate this approach, two examples of Greek constructivist educational software with science content are briefly presented: "Interaction between Objects", which aims at promoting knowledge construction about mechanical interaction and Newton's laws through interactive simulations of real-life situations and cognitive conflict processes, and "M.A.TH.I.M.A.", aiming at promoting construction of multiple, linked appropriate representations about several science topics (free-fall phenomenon, geometrical optics, heat and temperature, electric circuits, molecules and atoms). The proposed approach intends to enhance collaboration between software designers, content education research specialists, teachers and learners, in order to improve the quality of educational software to better respond to students' learning with understanding.
\end{abstract}

Key words: interactive educational software, constructivism, students' conceptions, software design, software evaluation, conceptual change

\section{Introduction}

The last years we witness the information explosion and the enormous impact of Information and Communication Technologies (ICT) in everyday life, work, and learning. Computers have great potential as cognitive tools (Jonassen, 1993). However, these tools can only enhance student achievement if appropriately used (Cognition and Technology Group at Vanderbilt, 1997; Bransford, Brown \& Cocking, 2000).

Technology-mediated learning is well investigated, especially in the higher education level to promote students' active learning, qualitative reasoning and conceptual understanding (Jonassen, Mayes \& McAleese, 1993; Kanuka \& Anderson, 1999). Secondary and primary educational levels should also benefit from those potentials.

In the past, usual teaching and instructional design were typically focused on the teacher planning and leading students through a series of instructional sequences and events to achieve a desired learning outcome (Gagné, Briggs \& Wager, 1988). Typically these forms of 
teaching refer to organized transmission of a body of knowledge followed by some forms of interaction with the material to consolidate the knowledge acquisition (Hedberg, Oliver, Harper, Wills \& Agostino, 2002). Also, technology-enhanced learning models have historically been developed around the transmission and retention of information through taught knowledge and skills, through the de-contextualized acquisition of passive, inert knowledge, and by assuming that reading, watching videos or controlling a button on these page-turners constituted 'active learning' (Young, 2003). In many cases these models failed to recognize the need for application in practice in order to understand how to effectively utilize knowledge (Jonassen, 1994).

The emergence of constructivism as a new learning theory tends to make clear the limits of the 'instructivist' model of learning and to shape new promises to improve teaching and learning in school. It is accepted that the new learning technologies should be informed by constructivist approaches for learning and teaching. These approaches are student-focused rather than teacher-focused, foster student active participation rather than passive attendance, use a variety of instructional tools rather than only print material, promote communication and collaboration among students rather than individualistic and competitive work, and facilitate operational rather than rote learning.

This paper deals with the main issues of constructivist theory of learning, and its impact in the design of constructivist educational computer systems. First it discusses the main theoretical issues and principles of the constructivist approach to learning, and to constructivist technology-mediated learning. It points out the necessity to use several techniques for the investigation and analysis of students' existing conceptions, as well as strategies to cope with students' alternative conceptions, in order to help them construct scientific knowledge. Then it proposes an approach for applying constructivism in the design, implementation and evaluation process of multimedia educational software, which is based on research in students' existing ideas and conceptions about the software's content. This approach is compared to existing models of software design, and it is illustrated by the presentation of two examples of educational software with science content, "Interaction between Objects" and "M.A.TH.I.M.A.", which have been designed and evaluated according to the proposed approach. Finally, implications for further research and collaboration among the persons involved in this design and evaluation process are discussed, as well as implications for the design of other software environments for distance learning.

\section{CONSTRUCTIVISM AND LEARNING TECHNOLOGIES}

In order to address the issue of how constructivism can inform the design of a multimedia educational software package, it is necessary to outline the basic assumptions of this theory and in particular its relations to technologically informed systems for learning.

\section{Constructivist views of learning}

A wide variety of educational approaches claim to be constructivist. According to Kunz (2004), in many cases e-learning literature gives the impression that constructivism is a result of the introduction of Information and Communication Technologies (ICT) in education, but in reality constructivism has its roots back in the years 1920s and 1930s of the last century. The work of Jean Piaget put the foundations of this learning theory, extended later by the work of Leon Vygotsky and many more theoreticians. Piaget's 'genetic epistemology' suggested that knowledge acquisition occurs due to two complementary processes, 
assimilation (when new information is incorporated in the existing cognitive structure), and accommodation (when new information constrains the existing cognitive structure, provokes re-structuring and the formation of a new cognitive structure). The interactions of the child with the material environment play a crucial role in experience acquisition and knowledge construction, and in the development of symbolic entities including language (Piaget, 1929/1967). Vygotsky on the other hand emphasized the influence of social and cultural contexts in learning, cognitive development and knowledge building. He maintained that thought is interiorized language and introduced the concept of 'zone of proximal development', which highlights the fact that when the learner is offered guidance or collaboration $\mathrm{s} / \mathrm{he}$ is able to develop many more skills than s/he can achieve on her/his own (Vygotsky, 1934/1988; 1978).

Subsequent ideas, which contributed substantially to the development of constructivist learning theories, are: a) Ausubel's idea that the most important factor that influences learning is what the learner already knows. Ausubel advised the teachers to get informed about the learners' prior knowledge, in order to teach them appropriately (Ausubel, 1968), and b) Wallon's idea about the development of scientific thought as a process of evolution of syncretic thought towards categorical thought by the emergence of categories in a previously undifferentiated state of mind (Wallon, 1945/1989; 1970).

The constructivist theoretical paradigm has been formulated after an extent number of research studies brought to light students' alternative conceptions concerning several mathematics and science topics. Constructivist theories support that scientific knowledge is personally constructed by the active, collaborative, reflective involvement of the learner in the pedagogical process, during which s/he interacts with new information, material, tools, persons, and cultural means (Driver \& Oldham, 1986; Duit \& Treagust, 1986; Driver, 1989a, 1989b; Scott, Asoko \& Driver, 1992). Constructivist theories have developed various ideas and principles transferred from cognitive psychology, epistemology and history of science to the domain of learning. For example, an important concept adopted by constructivists, especially of French origin, is 'cognitive obstacle', derived from the concept of 'epistemological obstacle' first introduced by Bachelard (1938/1993). This concept implies the idea that knowledge is constructed by means of discontinuities and cut-offs against common, everyday knowledge, bypassing the obstacles in its course. Common ideas, which may constrain this progress, constitute epistemological obstacles. Science education in particular used the concept of 'cognitive obstacle' or 'didactical learning obstacle' to describe several hard-core conceptions which hardly change with ordinary teaching and require special didactic approaches. In order to overcome those cognitive obstacles, specific pedagogical goals are necessary to define for constructive teaching ((aim-obstacle') (Martinand, 1986).

A number of noteworthy constructivist theories made important theoretical points about the use of constructivism in teaching and learning. In their review, Kanuka and Anderson (1999) remind us of the following theories: cognitive constructivism (knowing is an actively constructed individual thought process), radical or critical constructivism (reality is only a speculation, or a supposition, or a function of the workings of our cognitive structure and thus a very personal experience), situated constructivism (we can know only what is real; knowledge is grounded in the experience; the process of constructing knowledge involves examining and understanding the experience where the process occurs), and social constructivism (knowledge is an active process of constructing meanings socially through language and sharing cultural practices). Despite the differences of these stances, each constructivist approach has underlying similarities. Common to each stance is a belief that we construct knowledge based on what we already know (children's mind is no tabula rasa) and 
that learning is an active process of construction rather than a passive process of transmission of knowledge.

Thus, the importance of students' prior conceptions and knowledge and their active involvement in the learning process is crucial for appropriate knowledge construction. A constructivist approach for teaching and learning could be considered as a methodological tool serving a double purpose: the decision-making about the content of the knowledge to be taught and the design of the learning sequences and tasks (design of learning scenarios, simulations, activities, representations, questions, help, feedback, evaluation items, metalearning strategies, etc.). For this purpose, data issued from three different types of analysis should be considered:

i) Conceptual analysis of students' prior ideas, conceptions, knowledge, skills, models of reasoning, etc., as well as analysis of the actual scientific knowledge in the domain under study may reveal how important is the distance between the two models of thought, the everyday empirical and the scientific thought (Driver, 1989a, 1989b; Martinand, 1986).

ii) Epistemological analysis of the taught knowledge may identify conceptions, ideas, models of reasoning, etc., which have been developed during the historical evolution of scientific ideas. This study may reveal ideas that have many similarities with students' conceptions, which are different from the scientific ones and hardly change with teaching, sometimes functioning as 'cognitive obstacles' (Bachelard, 1938/1993; Martinand, 1986).

iii) Psychological analysis of the existing and the desired cognitive structures may inform the design of the learning tasks. The intellectual tasks should facilitate the development of scientific thought, and more particularly, children's intellectual evolution from concrete operational towards abstract operational thought (Piaget, 1929/1967), the evolution of their thought and language skills by the help of actions scaffolding them in a 'zone of proximal development' (Vygotsky, 1978), and the evolution of their syncretic thought towards categorical thought (Wallon, 1945/1989; 1970).

Those types of analysis can inform the design of the teaching strategies and tools. More specifically, they may inform the design of the scientific content to be studied, the specific teaching goals, which should also cope with students' cognitive obstacles, and the specific cognitive tasks to be undertaken during the various learning activities. The combination of those types of analysis should lead to didactical transposition (Chevallard, 1985/1992), i.e. the transformation of the scientist's knowledge content in order to fit the learner's knowledge and conceptual needs. During construction of scientific knowledge, collaboration and communication fosters negotiation of meaning among co-learners and the teachers, the learners' language is enriched, various point of views clarified and discussed, and eventual cognitive conflict situations promote students' conceptual change.

Thus constructivism should deal with every stage of knowledge construction, and with a variety of learning activities, teaching materials and tools.

\section{Constructivist technology-mediated learning}

Since Seymour Papert (1980) declared that computers can be powerful mind tools for children's construction of knowledge, many constructivist learning theories became widely accepted in all fields of education, including the application of technology to teaching and learning. This interest is related to the capacity of computers to provide an interactive environment that creates "an effective means for implementing constructivist strategies that would be difficult to accomplish in other media" (Driscoll, 1994: 376). 
There is evidence to show that computer systems have the potential to alter the traditional forms of teaching and learning, and serve as cognitive tools (Jonassen, 1993). Especially multimedia educational applications present a considerable potential as cognitive tools, by showing, proposing and giving direct evidence to the learners allowing them to see, observe, interpret, reflect, seek for direct evidence, and link the acquired experience to prior knowledge through animations, simulations, verbalizations, problem based scenarios, project based learning databases, multiple representations, team-based and collaborative learning (Roblyer, 1996; Hannafin, Hill \& Land, 1997; Waern, Dahlqvist \& Ramberg, 2000). Thus interactive multimedia technology could serve as a vehicle for constructive learning.

In their manifesto for a constructivist approach to technology use in higher education, David Jonassen, Terry Mayes and Ray McAleese (1993) found that the constructivist roles of technology in education depend on the use of various environments that represent multiple realities, promote case-based learning with real world tasks and environments, and facilitate collaborative knowledge construction. According to the authors, cognitive learning tools are all those that assist learners in representing their own knowledge or alternative representations of the external world, and computer-based applications that can function as cognitive tools including database managers, semantic networking programs, hypertext, spreadsheets, expert systems, and microworlds (Jonassen et al., 1993).

Though Jonassen and collaborators (1993) point out that knowledge construction cannot be achieved with all those computer applications (for example with browsing information systems). The process of knowledge construction would require specific instructional goals of the learning tasks, for which properly developed cognitive schemata have been developed. Those cognitive schemata are scientific ones, only if they have been formed and tested by use of scientific methods and tools.

Another problem arisen is that although too many ideas have been developed and expanded about constructivism and its relation to technology, this learning theory has not yet influenced educational technology systems design and implementation. As Cobb (1999: 15) stated, 'up to now a role for constructivism has been discussed more in principle than in practice, and claims about the kinds of knowledge it produces remain largely untested'. A number of researchers have been aware of this problem. For example, Kunz (2004) states that learning management systems have considerably delayed the application of constructivist approaches to the delivery of taught knowledge. The author proposes that the next generation of those systems should be based on principles obtained from the main practical educational applications of the constructivist learning approaches (Kunz, 2004), such as: cognitive apprenticeship (Collins, Brown, Newmann, 1989), collaborative knowledge building communities (Scardamalia \& Bereiter, 1994), goal-based scenarios or scenario-based design (see e.g. Carroll, Rosson, Chin \& Koenemann, 1998) to deal with complex real situations, and constructivist learning environments based on activity theory (Engestrom, 1987; Engestrom et al., 1999).

In fact, many educational technologies, more or less advanced ones, such as multimediahypermedia applications, intelligent tutoring systems, learning management systems, artificial intelligence and adaptive learning systems claim to support teaching and active learning. A problem that exists with those systems is that, despite their considerable potential in education, the majority of them tend to use more traditional pedagogical views and methods. For example, since 1989 the Organization for Cooperation and Development in Education (OCDE, 1989) had pointed out the lack and need for quality multimedia educational software. Nowadays, many software packages have been produced, but their quality may not always be as high as expected. 
In an attempt to design educational software packages, which would be accessible through the internet, more recent advanced learning technologies such as Intelligent Tutoring Systems (ITSs) have been developed. But ITSs seem to be more tutor-centered and instructivist than student-centered and constructivist. In order to offer instruction, ITSs develop architectures which are characterized by models of (Akhras, Self, 2002; Stauffer, 1996): a) the domain knowledge which represents the expert knowledge to be learned, organized as a set of correct production rules having a certain structure, b) the learner's knowledge that represents the correct and incorrect knowledge that the learner has about the domain; each new learner requires an individualized student model; in developing the student model, the type of knowledge (i.e. declarative, procedural) is determined, and c) the teaching knowledge, which represents the teaching strategies used by the ITS to select tutorial activities, present them to the learner and handle the learner's response.

Moreover, in order to assist students by scaffolding them in learning, these systems often develop a student model based on the learner's typical knowledge about the domain knowledge (e.g. novice, advanced) or her/his actions within the software (e.g. time on task, number of trials), to subsequently offering guidance towards specific instructional targets. Usually those targets follow traditional approaches leading the learner to the final goal through a series of steps. This final goal is defined in terms of a specific behavior the learner must demonstrate.

This approach does not take into account the individual learner's differences regarding prior knowledge or present motivation. It may be effective for procedural knowledge, which can be exhibited, but is not as effective with declarative knowledge, and higher levels of learning (Stauffer, 1996). Thus, advanced technological platforms for instruction hardly allow room for critical thought, active participation, operational learning and -finally- construction of appropriate scientific knowledge.

On the contrary, constructivist approaches to learning investigate and take into account students' existing conceptions, ideas, conceptual needs about the knowledge domain, also promoting the students' active role in learning. Technology-enhanced, student-centered learning environments organize interrelated learning themes into meaningful contexts; they provide interactive, complementary activities that enable individuals to address unique learning interests and needs; they study multiple levels of complexity, and deepen understanding (Hannafin \& Land, 1997).

As a consequence, constructivist views may lead to specific architectures of ITSs. For example Akhras and Self (2002) proposed a constructivist architecture of student-centered ITSs and emphasized different values from the traditional ITSs, in terms of knowledge representation, reasoning, and decision-making capabilities of the system. However, the authors illustrate their position by using a rather irrelevant example (making of salad), which has been criticized by other researchers (see e.g. Azevedo, 2002). Azevedo (2002) supported a quite different position that intelligent and adaptive learning environments can be used as meta-cognitive tools to foster self-regulating learning, and thus enhance learning. Young, DePalma and Garrett (2002) also criticized the position of Akhras and Self (2002), and maintained that computers should incorporate factors not only from the individual, but also constraints from the environment in her/his current situation. Despite the different views about the architecture of advanced computer systems, student-centered constructivist learning environments are generally considered as powerful technology-enhanced systems which can act as cognitive tools and foster active learning, critical thinking and higher-order skills (Jonassen, 1993; Duffy \& Cunningham, 1996; Hannafin \& Land, 1997; Wilson, 1998; Hedberg et al., 2002; Kunz, 2004).

Moreover, a complementary relationship appears to exist between computer technologies and constructivism, the implementation of each one benefiting the other, as the focus of both 
constructivism and technology are on the creation of new learning environments. A review of the literature on the implementation of computer technology in the classroom revealed that the connection between technology and constructivism lays on considering technological means as cognitive tools, which are able to foster higher order cognitive skills, when they are used by teachers having new roles within technology enhanced environments (Nanjappa \& Grant, 2003).

Thus, bringing constructivist principles into the classroom has implications for the learning environment, as well as teachers' and students' roles. The idea of the learning environment fits better with the idea of learning as a process of knowledge or meaning construction, which occurs by the help of multiple and continuous interactions between the person who learns and the means and persons of her/his environment (Perkins, 1998). According to Wilson (1998: 5) a learning environment is 'a place where the learners may work together and support each other as they use a variety of tools and information resources in their guided pursuit of learning goals and problem solving activities'. The constructivist view of learning emphasizes students' active involvement in the learning activities, collaboration among them and students' interactions with a variety of information resources, in order to construct meaning through experimentation, acquisition of empirical experience and appropriate pedagogical guidance (Edelson, Pea \& Gomez, 1998). The innovative use of computers in the classroom leads to important changes of the traditional roles of all the partners involved in the teaching and learning process. Within a new constructivistcollaborative learning environment students are no more patient receivers of knowledge, but active and responsible partners of the construction of their own knowledge, working either in small groups -or individually. Teachers are no more the unique owners and emitters of information and knowledge, but conceivers and designers of students' learning activities, and students' guides and assistants in the learning process.

It is evident that the simple presence of computers in the classroom could not result in such radical changes concerning teachers' and students' roles and teaching methods. Teachers' prior practices and routines influence changes teachers make in their classroom to accommodate technology. In fact, teachers tend to modify the technology to fit their teaching styles rather than modify their teaching style (Miller \& Olson, 1994). A possible solution to this situation would be the participation of teachers in appropriate education and training programs aiming at promoting the development of innovative and constructivist teaching strategies with the use of ICT (Sanholtz, Ringstaff \& Dwyer, 1996). Such training programs should assist and observe teachers in their own classroom, as we know too little about computer activities in the classroom and we need to understand the reality of ICT use in the educational context (Hinostroza, Rehbein, Mellar \& Preston, 2000).

\section{A CONSTRUCTIVIST DESIGN AND EVALUATION APPROACH BASED ON RESEARCH IN STUDENTS' IDEAS}

Within the social constructivist framework, research in students' initial ideas, conceptions, conceptual difficulties and needs constitutes an essential dimension which allows the collection of data concerning the students' initial conceptual state and its evolution over time. Research may also allow studying the contribution of specific teaching strategies in students' conceptual change.

We propose a research-based approach for the design, implementation and evaluation of constructivist educational software environments in three stages: research into students' 
conceptions, and conceptual needs, design of the software, and implementation and evaluation of it. More specifically, the three stages of this approach are the following:

\section{- Research into students' ideas and analysis of their conceptual needs}

The research in the first stage aims at investigating and studying students' initial empirical conceptions about the knowledge domain under study. Phenomenographic approaches (Marton, 1981, 1986) and methods such as personal interviews, written questionnaires, thinking aloud protocols, drawings tasks, etc. can be used to explore students' existing conceptions and conceptual difficulties and needs at the initial stage of software development. Research with representative sample of students may provide with data useful also in case that the students that are going to use the software are different from the initial sample.

This process differs to some extend from what is already done as 'requirements gathering' phase in current educational software development. As Carroll and collaborators (1998) state, use cases (specify sets of possible event traces but do not describe user experiences and motivations; cooperative design scenarios are used to characterize work flow and breakdowns and "are used as conversational props in user-developer workshops, but are not cognitively articulated (in terms of user goals, expectations, and reactions) and are not taken as scoping contexts for design rationale" (Carroll et al., 1998: 1157). In the scenario-based approach to requirements development by Carroll et al. (1998), the 'design team' consists of middle school and high school teachers, human-computer interaction specialists and software technologists. Ethnographic methods are used to collect and analyze data from real classrooms and laboratory activities in order to design and develop scenario-based software relative to a virtual science laboratory. In this approach, students' existing ideas, conceptions, conceptual difficulties and needs are not investigated nor taken into account in the design process, although many important students' alternative conceptions may exist relative to the studied science topics.

Also, this first step of the proposed research-based approach differs significantly from instructional design in that it stresses the exact characteristics of the learners and the learning goals. For example, Liu \& Johnson (2003) propose a new approach to design technology systems based on instructional design principles (Reigeluth, 1983/2002; Smith \& Ragan, 1993). In this approach an effort is made to integrate and analyze all the factors that may play a significant role in the pedagogical process. This three-dimensional approach includes information, technology and instructional design. Regarding the latter, four major phases are viewed and crucial factors in the first Planning Instruction phase are the analysis of "content, learners and tasks" (Liu, Johnson, 2003: 1011). Though, the subsequent described analysis does not make reference to students' prior ideas as a crucial factor to analyzing and decisionmaking of learning content, tasks, technologies, which are afterwards proposed.

In addition, design methods adopting the activity theory are often focused on factors relative to Human-Computer Interaction (Nardi, 1996) or can investigate the implementation of ICT in real school settings (Romeo \& Walker, 2002), without focusing on students' ideas. So the usual design methods do proceed to requirements of teaching, yet without analyzing students' thought and conceptual difficulties.

\section{- Design}

The second stage of the method includes the design and development of the software on the basis of the results issued from the initial research concerning students' empirical ideas, conceptual difficulties and needs. The analysis of research data may serve as a guide for the selection of both the content to be taught and the learning tasks to be proposed. A step-by-step 
design of the software aiming to help students change their alternative conceptions and overcome their conceptual difficulties may result in the creation of a constructivist-based electronic tool characterized by a number of features (Jonassen, 1994). More specifically, the software should provide:

- proper content, after analysis of students' conceptions and needs, as a result of didactical transpositions (Chevallard, 1985/92),

- construction of operational content -and context- dependent knowledge,

- multiple linked representations of the complex reality,

- simulations of 'real' situations (Brown, Collins \& Duguid, 1989), and authentic tasks in meaningful contexts (Roth, 1995),

- case-based learning, problem-solving situations, thoughtful reflection on experience,

- proper feedback and guidance to confront students' alternative ideas,

- opportunities for collaboration, communication and social negotiation of meaning among learners,

- representations, symbols, language and tasks promoting equity of students of both genders and belonging to various socioeconomic and cultural environments (Solomonidou, 2001/2007).

Sometimes, it is necessary to assign a number of 'aims-obstacles' for the software pedagogical design, especially of science content. The software should provide students with many opportunities to express and evaluate their personal ideas and lead them to 'cognitive conflict' situations, in order to provoke conceptual change and facilitate construction of scientific knowledge. Formative evaluation of the software with a small number of students and teachers may provide elements regarding the ease of its use, and appropriateness of the software's interface, knowledge content and learning process.

This second step of the proposed approach differs from most well known models of designing distance education and e-learning programs, which are oriented to the delivery of rules and processes, do not adopt contemporary educational views and aim at the transmission of knowledge (Pantano-Rokou, 2005).

\section{- Evaluation and implementation}

A third stage of the approach is finally conducted aiming at the summative evaluation of the software after its use in class conditions and its implementation in real classroom settings. Guiding lines may again be students' ideas, conceptions, eventual conceptual change, and learning with understanding. Also, teachers' opinions about the effectiveness of the software's use in teaching should be included in this stage. Post-test written questionnaires and personal interviews with students and teachers may be used to select data for the evaluation of the learning outcomes, and the software's summative evaluation.

The proposed method seems to be a quite complex task, demanding the collaboration of designers, researchers, and teachers. It would be rare for one single person to integrate all those roles. Therefore, teamwork is necessary between various persons who may have distinct roles in each stage.

- During the first stage of initial research, the researcher has the predominant role assisted by the teacher and the designer in investigating and studying students' empirical conceptions, as well as in analyzing the didactical transformations to define the software's content. 
- In the second stage, the designer plays a crucial role in designing the computer environment (i.e. simulations, visualizations, interface) and is assisted by the researcher and the teacher in the design of the pedagogical material to be included in the software (i.e. what kind of activities, tasks, working sheets, questions, feedback, evaluation items,...).

- In the third stage, the teacher takes charge of the pedagogical situation, as s/he organizes the new ICT learning environment to implement the new pedagogical tool. In this stage, the researcher is involved in designing and conducting the research and study students' final conceptions and learning outcomes. The designer focuses on rather technical aspects and aspects regarding the interaction of students with the software they used it. The gathering and study of the research data leads to the summative evaluation of the software.

The novelty of our approach is that all the stages of the usual approach, which has: analysis $>$ design $>$ implementation $>$ evaluation, the students' conceptions, ideas and conceptual needs are the guiding line. Also in our approach, besides the usual persons involved, that is: users/learners, domain experts, designers and programmers, content education researchers are actively involved in investigating and analyzing the students' conceptual state regarding the content of learning in every stage. Thus in the initial research and analysis stage, the design stage and the implementation and evaluation stage not only the usual specialists, but also content education specialists are involved.

Diagram 1 summarizes the research-based approach to the constructivist design, evaluation and implementation of educational software. It outlines the main and assistive roles of the researcher, the designer and the teacher, as well as the main tasks in every stage of research. 
Diagram 1. An approach to constructivist design, evaluation and implementation of educational software based on research in students ideas
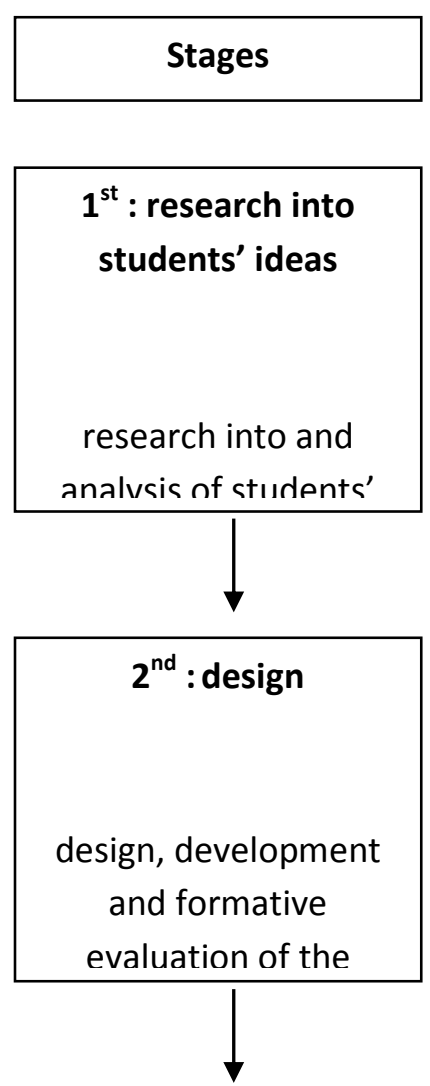

$3^{\text {rd }}$ : evaluation

implementation and summative evaluation

of the software
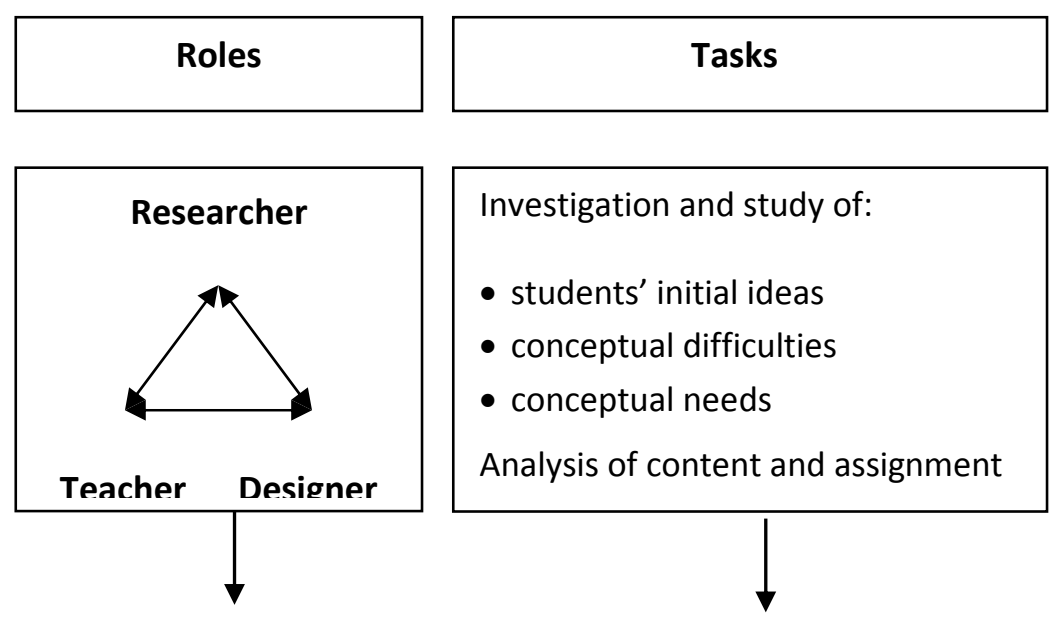

Investigation and study of:

- students' initial ideas

- conceptual difficulties

- conceptual needs

Analysis of content and assignment

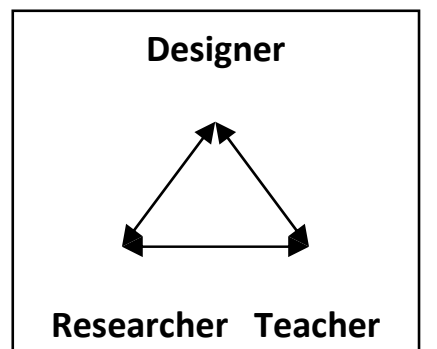

Design of the software's:

- content (according to students' existing ideas)

- process (simulations, learnung scenarios, tasks, feedback, etc.)

- interface
- Teaching with the software in class settings.

- Research on students' final ideas and conceptual change, as well as of teachers' opinions.

- Software summative evaluation after analysis of research data. 


\section{EXAMPLES OF CONSTRUCTIVIST EDUCATIONAL SOFTWARE}

In order to illustrate the described research approach, two representative examples of constructivist interactive multimedia educational software with science content designed and evaluated in Greece are presented.

\section{$1^{\text {st }}$ example: "Interaction between Objects"1}

The software "Interactions between Objects" aims to support effective teaching and learning regarding Newton's $3^{\text {rd }}$ law and Newtonian Dynamics. The reason to proceed to the creation of this educational package was the significant number and persistence of students' alternative ideas about this science area revealed by a large amount of research studies, and the insufficient emphasis usually science teaching gives in students' ideas regarding Newton's third law (Viennot, 1979). In order to design and evaluate this software an extended research has been conducted involving science education researchers, designers, programmers, students and teachers in the three stages.

The research in the first stage investigated Greek students' initial ideas about the concept of interaction between objects with 10 clinical-type personal interviews (Solomonidou \& Kolokotronis, 2001), pre-test questionnaires answered by 451 students, and finally personal interviews with another 26 students of the above sample. The results showed an important divergence (about 70\%) between students' empirical ideas and the relevant scientific views and also differentiations concerning students' answers associated with gender, age, school, and area of residence.

The second stage included the design and development of the software on the basis of students' empirical ideas. In order to help students change their initial empirical conceptions into scientific ones, the software simulates real everyday life situations of interaction between objects and models those situations according to the method of extended figures first introduced by Viennot (1979). A powerful feature of the software student is 'run-my-model' processes (Raghavan \& Glaser, 1995), as it allows the student to create her/his own model of reality according to her/his ideas and then activate this personal model through an appropriate simulation (in Figure 1 an example is shown). Then the comparison of the student's personal model to the scientific one may eventually lead her/him to conceptual change and construction of scientific views (the software design is described in Kolokotronis \& Solomonidou, 2003). The second stage also included a small-scale research for the software's formative evaluation with the help of 8 students and 15 teachers. The students were videotaped while they were working with the software and then participated in personal interviews. The teachers worked with the software and then filled in an evaluation questionnaire. The analysis of the data showed that the software's interface, experiments and tasks were especially attractive to the students, and also contributed to their conceptual change. The teachers evaluated the software as "very good' and made comments regarding aspects of its design, which contributed to its improvement.

In the third stage of research, the software has been implemented in 13 primary and secondary school classes and used in teaching interventions with 226 students. Data have been collected and analyzed in order to perform a summative evaluation of the software. More particularly, the teachers filled in a "diary" in order to report on the attitude and the reactions during teaching of both the whole class and a small group of 2-3 students. Two weeks after teaching, the 226 students answered a post-test written questionnaire similar to the pre-test one. The study of the teachers' reports revealed that the students' attention, active participation and collaboration were significantly more important compared to traditional 
teaching (Solomonidou \& Kolokotronis, 2004). Also, the comparison of the students' answers to the pre- and the post- test questionnaire showed that the percentages of students' incorrect answers had substantially decreased (from $60 \%$ to $90 \%$ ), and that the initial differences associated with gender, age and area of residence have diminished.

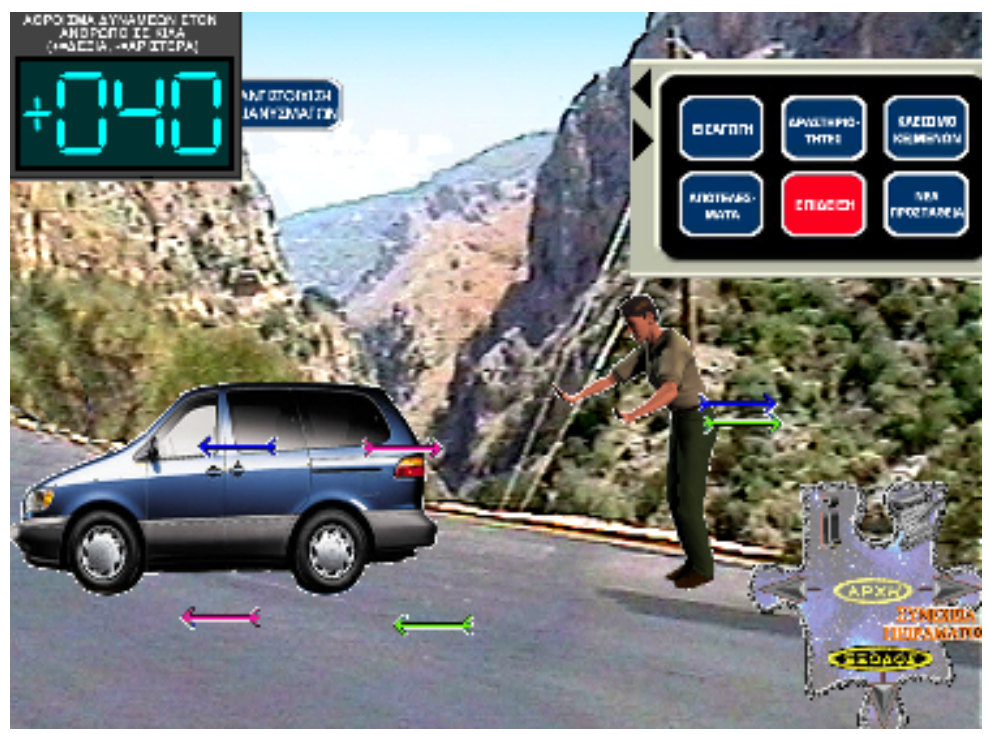

Figure 1. "Interactions between Objects": the driver starts moving backward while trying to move his car forward ('run my model')

\section{$2^{\text {nd }}$ example: "M.A.TH.I.M.A."}

"M.A.TH.I.M.A." is a highly interactive multimedia educational software package aiming to help students construct multiple linked representations and foster conceptual understanding in several science topics (free fall phenomenon in mechanics, geometric optics, heat and temperature, electric circuits, molecules and atoms). The design of the software was based on the study of science education literature concerning students' conceptions and difficulties about the thematic areas developed. For example the literature reviews on students' ideas about the free fall phenomenon (Driver et al., 1994) served to the design of the thematic unit 'Mechanics'. In this unit the student can study the free fall phenomenon by running simulations either of natural environments (on the earth's or the moon's surface), or alternative worlds (earth without atmosphere). The falling of an object is studied by tracking the objects' motion, showing a dynamic model of the fall, and dynamic graphs of the evolution in time of vector entities.

The 'Reflection-refraction' unit has been developed on the basis of students' various alternative ideas about light (Driver et al., 1985). This unit simulates a Geometric Optics laboratory where the student is engaged in problem solving activities, such as predicting the result of an experiment related to linear diffusion of light, shadows formation, reflection and refraction, synthesis of color light beams, and observe a highly dynamic and interactive geometric model of the situation under study. S/he can also enjoy a game with little mirrors and diamonds (Solomonidou et al., 2000).

Concerning Molecules and Atoms, an amount of research studies revealed students' alternative conceptions about the particulate nature of matter and their idea of matter as a continuous and static medium (Stavridou, 1995). In order to overcome students' confusion between scientific view of matter's structure and their conception as deriving from everyday 
experience, the unit 'Heat and Temperature' has been developed in order to promote modelization of appropriate phenomena in the microscopic level. Apart from students' difficulties about the particulate nature of matter, this unit aims at coping with their alternative ideas concerning heat and temperature. The learning environment of this unit simulates a science laboratory, where students are engaged in experiments related to the thermal expansion of solid, liquid or gaseous materials, and to the change of water from solid to liquid and then to gaseous state, and subsequently are introduced to the microscopic models of matter through appropriate modelization tasks (Stavridou, 1995). The student interacts with simulations of several phenomena while the screen may display both a simulation of the relevant experiment in microscopic level and a dynamic graph of the temperature change (Stavridou et al., 2000). Figure 2 shows a screen referring to thermal expansion of a liquid: the left window shows the particles' motion when temperature increases, the central window shows a dynamic graph of the volume change as temperature increases, next the experimental set is shown and on the right there are relevant questions and tasks, aiming to enhance conceptual understanding.

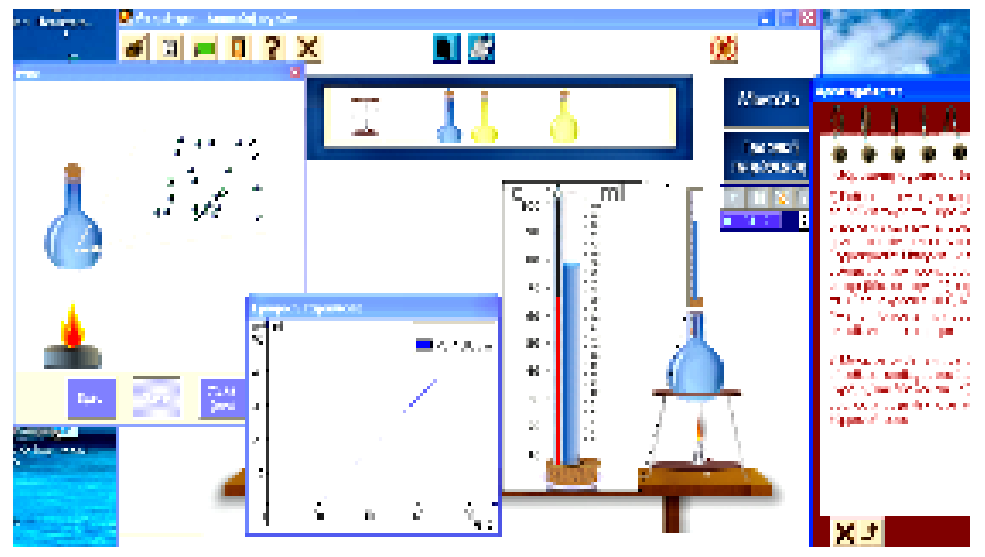

Figure 2: "M.A.TH.I.M.A.”: Study of thermal expansion of a liquid

Regarding Electricity, students' alternative conceptions related to electric current (Driver et al., 1985) have been taken into account. The environment here simulates a laboratory providing the students with materials and appliances to allow them get actively involved in experiments in which they manipulate elements and values of entities of electric circuits, and also dynamic simulations of those circuits in microscopic level, in order to help them understand basic electricity concepts (Samarakou et al., 2000).

During the design and development of the software, this was constantly tested in a large Greek secondary school (a science teacher of this school was a member of our design team). The results of the tests during the software's formative evaluation were positive, and the teachers' and students' comments were utilized in order to improve it. After its completion, "M.A.TH.I.M.A." has been presented to teachers during a number of conferences and seminars and implemented in several schools. After its use it has been evaluated with a written questionnaire answered by a number of secondary and primary teachers. Those teachers reported that the software: a) is a highly interactive and user-friendly one with a pleasant interface and simulations of various experiments, $b$ ) improves the learning outcomes in every unit it is used, c) contains simulations which promote conceptual understanding, d) "it constitutes a very good learning tool", and e) helped both the teachers to save time in 
experimentation, and the students to improve their attitude toward science, as well as to assure their active involvement in teaching.

\section{Conclusion}

The paper proposes a constructivist approach to the design, implementation and evaluation of multimedia educational software. Central to this constructivist approach is the idea that students' existing ideas and conceptions play a crucial role in learning. Many advanced computer-learning systems do not take this assumption into account, and tend to use more traditional pedagogical views of knowledge transmission. The need for the creation of constructivist computer systems has been discussed, as well as the importance of a constructivist-collaborative learning environment to foster students' conceptual understanding (Wilson, 1998; Perkins, 1998). Due to their specific interactive features computers are considered as powerful mind tools or cognitive tools (Papert, 1980; Jonassen, 1993; Duffy, Cunningham, 1996), which can promote operational and active learning, and they can implement constructivist strategies that would be difficult to accomplish in other media (Driscoll, 1994).

In order to illustrate the proposed research method, two examples of Greek educational multimedia software with science content were briefly presented, and more specifically, "Interactions between Objects" aiming at to promoting construction of scientific knowledge in the area of Newton's $3^{\text {rd }}$ law and Newtonian Dynamics, and "M.A.TH.I.M.A.", aiming at helping students construct multiple linked representations and conceptual understanding in several physics areas.

But a problem that exists is that attempts like the ones described in this paper tend to remain restricted to their local context with few chances for broader dissemination. As Kunz (2004) pointed out, many of the advanced systems are commercially available, while others are in-house products developed mainly by groups of researchers, more often working at universities or other institutions. The former are well known, accessible and usable via the Internet, whereas the latter are not well known to the large public. Educational software packages inspired by constructivist views have not been widely spread distributed. The position of this paper is that constructivist theory and research into students' ideas and conceptual evolution should inform on more extensive scale both the design of educational software packages and their implementation in schools and evaluation. In addition, the presented approach proposes a broader collaboration of several specialists coming from different science areas, in order to improve the teaching process and learning outcomes. An important perspective of this work would be the appropriate -technical- design which would permit constructivist educational software packages to be introduced in the web, in order to serve as useful distance and open learning tools for a larger number of students, teachers, practitioners and researchers.

\section{NOTES}

1. The software "Interactions between Objects" has been designed by the author in collaboration with Dr D. Kolokotronis, developed at the Educational Technologies and Software Design Laboratory (University of Thessaly, Greece), and evaluated by Dr Kolokotronis during his doctoral thesis elaboration at the University of Thessaly (1999-2002).

2. The software 'M.A.TH.I.M.A.' was designed and developed by a group of researchers coordinated by Associate Professor M. Grigoriadou (University of Athens). The project was financed (1998-1999) by the 
Open Education - The Journal for Open and Distance Education and Educational Technology Volume 5, Number 1, 2009 @) Open Education

ISSN: 1791-9312

Greek Ministry of Education (project "Reformulation and Innovation of the Curricula in Science through Production of Instructional Material" - EPEAEK, E22), and supported by the Pedagogical Institute.

\section{ACKNOWLEDGEMENTS}

The author would like to gracefully thank Dr Fotini Stavridou for her useful corrections on the manuscript. 


\section{References}

Akhras, F.N., Self, J.A. (2002) 'Beyond intelligent tutoring systems: Situations, interactions, processes and affordances', Instructional Science, 30 (1), pp. 1-30

Ausubel, D.P. (1968). 'Educational psychology: a cognitive view', New York, Holt, Reinhart \& Winston

Azevedo, R. (2002) 'Beyond intelligent tutoring systems: Using computers as metacognitive tools to enhance learning?’ Instructional Science, 30 (1), pp. 31-45

Bachelard (1938/1993: 15th édition) 'La formation de l'esprit scientifique', Paris, Librairie Philosophique Vrin

Bransford, J., Brown, A., Cocking, R. (eds.) (2000) 'How people learn: brain, mind, experience, and school', Washington, D.C., National Academy Press

Brown, J.T., Collins, A., Duguid, L. (1989) 'Situated cognition and the culture of learning', Educational Researcher, 18, pp. 32-43

Carroll, J., Rosson, M.B., Chin, G. Jr., Koenemann, J. (1998) 'Requirements development in scenario-based design', IEEE Transactions on Software Engineering, 24 (12), pp. 1156-1170

Chevallard, Y. (1985/1992) 'La transposition didactique', Grenoble, La pensée sauvage

Cobb, T. (1999) 'Applying constructivism: A test for the learner-as-scientist', Educational Technology Research \& Development, 47 (3), pp. 15-33

Cognition and Technology Group at Vanderbilt (1997) 'The Jasper project: lessons in curriculum instruction, assessment, and professional development', Mahwah, NJ, Lawrence Erlbaum Associates

Collins, A., Brown, J.T., Newman, S.E. (1989) 'Cognitive apprenticeship teaching the crafts of reading, writing and mathematics', In L.B. Resnick (ed.) Knowing, learning and Instruction: Essays in Honor of Robert Glaser, Mahwah, NJ, Lawrence Erlbaum Associates, Publishers

Driscoll, M.P. (1994) 'Psychology of learning for instruction', Toronto, ON, Allyn and Bacon

Driver, R. (1989a) 'Students' conceptions and the learning of science', International Journal of Science Education, 11 (5), pp. 481-490

Driver, R. (1989b) 'Changing conceptions', In P. Adey (ed.) Adolescent development and school science (pp. 79-99), London, Falmer Press

Driver, R., Guesne, E., Tiberghien, A. (eds.) (1985) 'Children’s Ideas in Science', London, Open University Press

Driver, R., Oldham, V.A. (1986) 'A constructivist approach to curriculum development in science', Studies in Science Education, 10, pp. 37-60

Driver, R., Squires, A., Rushworth, P., Wood-Robinson, V. (eds.) (1994) 'Making sense in secondary science. Research into children's ideas, London, Routledge

Duit, R., Treagust, D. (1998) 'Learning in science: from behaviourism towards social constructivism and beyond', In B.J. Fraser and K.G. Tobin (eds.) International Handbook of Science Education (pp. 3-25), Dordrecht, Kluwer Academic Publishers

Duffy, T.M., Cunningham, D.J. (1996) 'Constructivism: Implications for the design and delivery of instruction' In D.H. Jonassen (ed.) Educational communications and technology (pp. 170-199), NY, Simon \& Schuster Macmillan

Edelson, D., Pea, R., Gomez, L. (1998) 'Constructivism in the collaboratory’ In B.G. Wilson (ed.) Constructivist Learning Environments. Case studies in instructional design (pp. 151-164), Englewood Cliffs, NJ, Educational Technology Publications

Engestrom, Y. (1987) 'Learning by expanding: An activity-theoretical approach to developmental research', Helsinki, Orieta-Konsultit

Engestrom, Y. et al. (1997) 'Perspectives on activity theory', Cambridge, UK, Cambridge University Press

Gagné, R., Briggs, LJ., Wager, W. (1988) 'Principles of instructional design', New York, Horton Rinehart and Winston

Hannafin, M., Hill, J., Land, S. (1997) 'Student-centered learning and interactive multimedia: status, issues and implication', Contemporary Education, 68 (2), pp. 94-99

Hannafin, M., Land, S. (1997) 'The foundations and assumptions of technology-enhanced student-centered learning environments', Instructional Science, 25, pp. 167-202

Hedberg, J., Oliver, R., Harper, B., Wills, S., Agostino, S. (2002) 'Implementing generic designs upon quality ICT exemplars', In Kishnuk (ed.) Proceedings of the International Conference on Computers in Education (ICCE'02) (pp. 1011-1016), Los Alamitos, CA, USA, IEEE CS

Hinostroza, E., Rehbein, L., Mellar, H., Preston, C. (2000) 'Developing educational software: A professional tool perspective', Education and Information Technologies, 5 (2), pp. 103-117

Jonassen, D.H. (1993) 'Computers in the classroom: mindtools for critical thinking', Englewood Cliffs, New Jersey, Prentice Hall 
Jonassen, D.H. (1994) 'Thinking technology: Towards a constructivist design model, Educational Technology, 3 (4), pp. 34-37

Jonassen, D., Mayes, T., McAleese, R. (1993) 'A manifesto for a constructivist approach to technology in higher education', In T. Duffy, D. Jonassen, J. Lowyck (eds), Designing constructivist learning environments, Heidelberg, FRG, Springer-Verlag

Kanuka, H., Anderson, T. (1999) 'Using constructivism in technology-mediated learning: constructing order out of the chaos in the literature', Radical Pedagogy, 1 (1), 1999 . Available at: http://radicalpedagogy.icaap.org/content/vol1.1999/issue2/02kanuka1_2.html

Kolokotronis, D., Solomonidou, C. (2003) 'A step-by-step design and development of an integrated educational software to deal with students' empirical ideas about mechanical interaction', Education and Information Technologies, 8 (3), pp. 229-244

Kunz, P. (2004) 'The next generation of learning management systems (LMS): requirements from a constructivist perspective', In L. Cantoni, C. McLoughlin (eds.) Proceedings of ED-Media 2004-World Conference on Educational, Multimedia, Hypermedia \& Telecommunications (pp. 300-307), Lugano, Switzerland, 6/2004

Liu, L., Johnson, L. (2003) 'A new approach of design: technology integration as information system development', Technology and Teacher Education International Conference, 2003 (1), pp. 1010-1016

Martinand, J.-L. (1986) 'Connaître et transformer la matière', Génève, Peter Lang

Marton, F. (1981) 'Phenomenography - describing conceptions of the world around us', Instructional Science, 10, pp. $177-200$

Marton, F. (1986) 'Phenomenography - a research approach to investigating different understandings of reality', Journal of Thought, 21 (3), pp 28-49

Miller, L, Olson, J. (1994) 'Putting the computer in its place: A study of teaching with technology', Journal of Curriculum Studies, 26 (2), pp. 121-141

Nanjappa, A., Grant, M.M. (2003) 'Constructing on constructivism: the role of technology', Electronic Journal of Integrating Technology in Education. Recovered on 13th February, 2005 from http://ejite.isu.edu/Volume2No1/nanjappa.htm

Nardi, B. (1996) (ed.) 'Context and consciousness: activity theory and Human-Computer Interaction', Cambridge, MIT Press

OCDE/OECD, Centre for Educational Research and Technology (1989) 'Information Technologies in Education. The quest for quality software', Paris

Pantano-Rokou, F. (2005) 'Educational design for e-learning: Models and impact on learning', Open Education: The journal of Open and Distance education and Educational Technology, 1, pp. 45-68

Papert, S. (1980) 'Mindstorms, children, computers and powerful ideas', New York, Basic Books

Perkins, D. (1998) 'Minds in the "hood". Foreword', in B.G. Wilson (ed.) Constructivist Learning Environments. Case studies in instructional design', Englewood Cliffs, NJ, Educational Technology Publications

Piaget, J. (1929/1967) 'The child's conception of the world', London, Routledge

Raghavan, K., Glaser, R. (1995) 'Model-based analysis and reasoning in science: the MARS curriculum', Science Education, 79, pp. 37-61

Reigeluth, C. (ed.) (1983/2002) 'Instructional-design theories and models: An Overview of their Current Status', Hillsdale, New Jersey, Lawrence Erlbaum Associates. Inc

Roblyer, M. (1996) 'The constructivist/objectivist debate: implications for instructional technology research', Learning and Leading with Technology, October, 12-16

Romeo, G., Walker, I. (2002) 'Activity theory to investigate the implementation of ICTE', Education and Information Technologies, 7 (4), pp. 323-332

Roth, W.-M. (1995) 'Authentic school science', Netherlands, Kluwer Academic Publishers

Samarakou, M., Mitropoulos, D., Grigoriadou, M., Solomonidou, C., Stavridou, H., Rigoutsos, A., Mazarakos, S. (2000) 'Simulation of simple and complex electric circuits', in N. Valanidis (ed.) Proceedings of the 2nd PanHellenic Conference 'Science Education and Application of New Technologies in Education' (pp. 129-139), Cyprus, 5/2000 (in Greek)

Sanholtz, J., Ringstaff, C., Dwyer, D. (1996) ‘Teaching with technology’, USA, Teachers College Press

Scardamalia, M., Bereiter, C. (1994) 'Computer support for knowledge-building communities', Journal of the Learning Sciences, 3, pp. 265-283

Scott, P., Asoko, H., Driver, R. (1992) 'Teaching for conceptual change: a review of strategies', in R. Duit, F. Goldberg, H. Niedderer (eds.) 'Research in physics learning: theoretical issues and empirical studies' (pp. 310329), Germany, Institute for Science Education at the University of Kiel

Smith, P.L., Ragan, T.J. (1993) 'Instructional design', Upper Saddle River, NJ, Merrill

Solomonidou, C. (2001/2007 5th edition) 'Contemporary educational technology: computers and learning in the knowledge society', Thessaloniki, Kodex editions (in Greek) 
Solomonidou, C., Kolokotronis, D. (2001) 'Interactions between bodies: students' initial ideas and development of appropriate educational software', Themes in Education, 2 (2-3), pp. 175-210

Solomonidou, C., Kolokotronis, D. (2004) 'Looking beyond design and implementation stages: evaluating an educational software in class conditions', in L. Cantoni, C. McLoughlin (eds.) Proceedings of ED-Media 2004 (pp. 2468-2475), Lugano, Switzerland, 6/2004

Solomonidou, C., Stavridou, H., Grigoriadou, M., Mitropoulos, D., Rigoutsos, A., Samarakou, M. (2000) 'Light and colors: design and development of educational software to study Geometric Optics phenomena', in D. Meimaridou-Voulgaraki (ed.) CD-ROM Proceedings of the Pan-Hellenic Conference 'Informatics in Education', Thessaloniki, SEPDETH, 11/2000 (in Greek).

Stauffer, K. (1996) 'Student modeling and web-based learning systems', Athabasca University, Recovered on 13th February, 2005 from http://ccism.pc.athabascau.ca/html/students/stupage/Project/initsm.htm

Stavridou, H. (1995) 'Physical science models and learning processes', Athens, Savalas editions (in Greek)

Stavridou, H., Solomonidou, C., Samarakou, M., Grigoriadou, M., Mitropoulos, D., Rigoutsos, A. (2000) 'Educational software for the study and monetization of thermal expansion and change of matter state', in V. Komis (ed.) Proceedings of the 2nd Pan-Hellenic Conference 'Information and Communication Technologies in Education' (pp. 489-498) Patras, 10/2000 (in Greek).

Viennot, L. (1979) 'Spontaneous reasoning in elementary dynamics, European Journal of Science Education, 1 (2), pp. 205-221

Vygotsky, L. (1934/1988: Greek transl.) 'Thought and language, Athens, Gnosi editions

Vygotsky, L.S. (1978) 'Mind in society: the development of higher psychological processes', Cambridge, MA, Harvard University Press

Waern, Y., Dahlqvist, P., Ramberg, R. (2000) 'Learning contexts-Does multimedia affect physics learning?', in R.H. Sprague, Jr. (ed.) Proceedings of the Thirty-Third Annual Hawaii International Conference of System Sciences, IEEE Computer Society, Los Alamitos, California, 2000, Recovered on 31th January, 2005 from http://csdl.computer.org/comp/proceedings/hicss/2000/0493/03/04933039.pdf

Wallon, H (1970) 'De l'acte à la pensée', Paris, Flammarion

Wallon, H. (1945/1989) 'Les origines de la pensée chez l'enfant', Paris, Quadridge/PUF

Wilson, B.G. (1998, 2nd edition) 'What is constructivist learning environment? Introduction', in B.G. Wilson (ed.) Constructivist Learning Environments. Case Studies in Instructional Design (pp. 3-8), Englewood Cliffs, New Jersey, Educational Technology Publications

Young, L. (2003) 'Bridging theory and practice: developing guidelines to facilitate the design of computer-based learning environments', Canadian Journal of Learning and Technology, 29 (3), Available at http://www.cjlt.ca/content/vol29.3/cjlt29-3_art4.html

Young, M.F., DePalma, A., Garrett, S. (2002) 'Situations, interaction, process and affordances: An ecological psychology perspective', Instructional Science, 30 (1), pp. 47-63. 


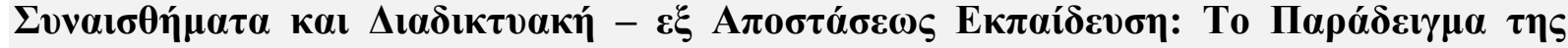 Kv́ $\pi \rho 0 v$ / Emotions and Online-Distance Education: The Case of Cyprus
}

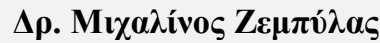

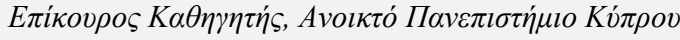 \\ e-mail: $\underline{\text { m.zembylas@ouc.ac.cy }}$

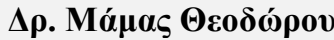

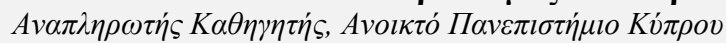 \\ e-mail:m.theodorou@ouc.ac.cy

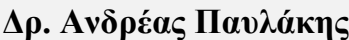

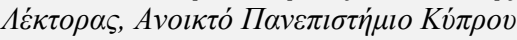 \\ e-mail:pavlakis@ouc.ac.cy
}

\begin{abstract}
This article examines the origin and impact of adult learners' emotions in the context of a distance learning program at the Open University of Cyprus. A range of methods for accessing emotions related to online learning is used, such as emotion diaries, semi-structured interviews, and email messages. The study highlights the multiple directions adult learners' emotions can take and the significance of learners' interpretations of their emotions in relation to the learning methodology. The issues of social and emotional communication and contact emerge as critical in the exploration of adult learners' emotions in the context of online learning. In particular, the study shows how adult learners' emotions in online learning are manifest and expressed in ambivalent ways, that is, how both positive and negative emotions co-exist and form particular emotional climates that influence adults' learning experiences throughout the course. Analyzing the data from this study confirms findings from other recent research that in order to decrease negative emotions associated with online - distance learning methodology, the learners must familiarise themselves with the technology at hand, enhance quality communication with fellow learners and instructors as well as become well-organised and manage their time effectively. In addition, the programme must be well-organised and the instructors must facilitate the learners' needs in a timely and organised manner. All in all, the current study shows that there is a lot to be gained from further considering how adult learners talk about their emotions as they learn how to become online learners.
\end{abstract}

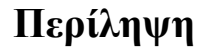

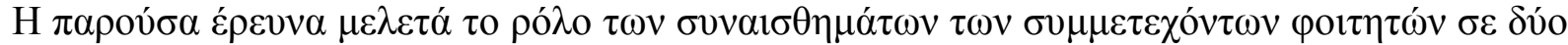

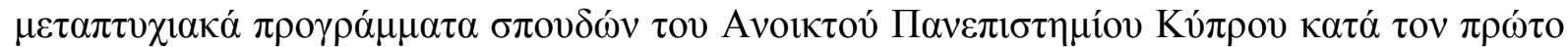

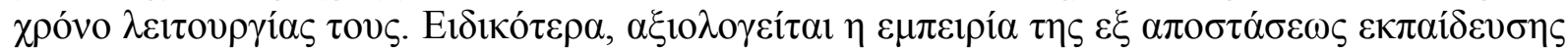

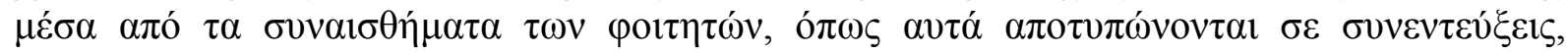

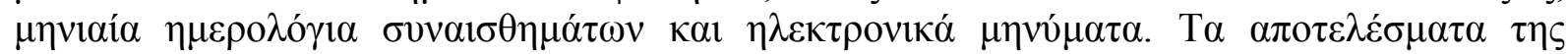

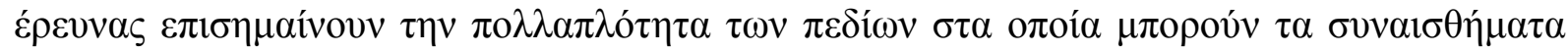

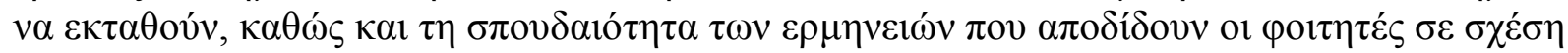

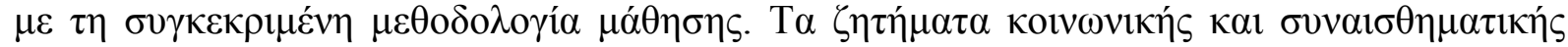

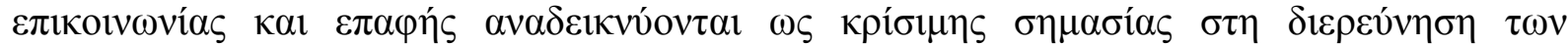

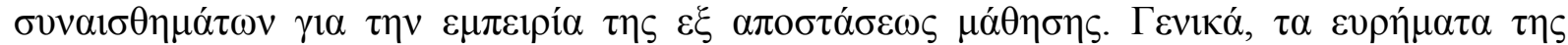

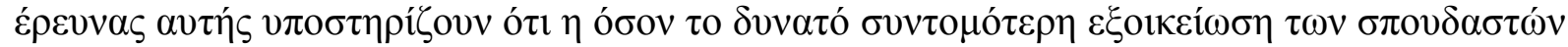

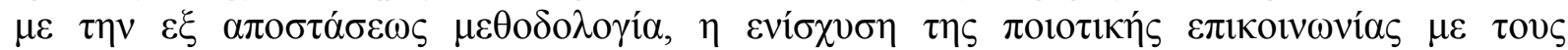




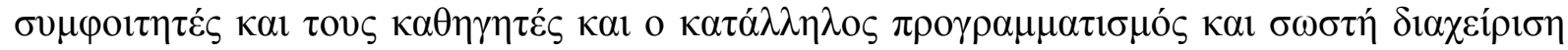

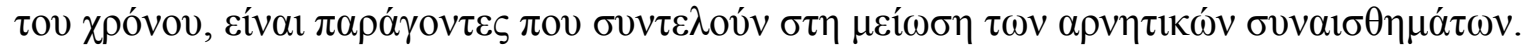

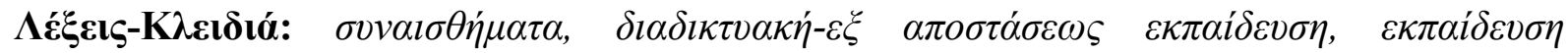
$\varepsilon v \eta \lambda i \kappa \omega v, \varepsilon ́ \rho \varepsilon v v \alpha \delta \rho \alpha ́ \sigma \eta \varsigma, K \dot{\pi} \pi \rho \varsigma$

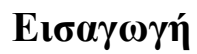

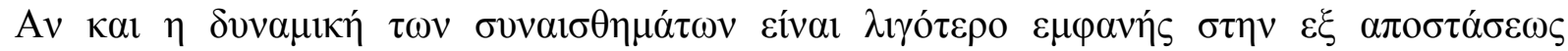

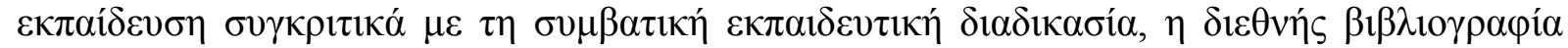

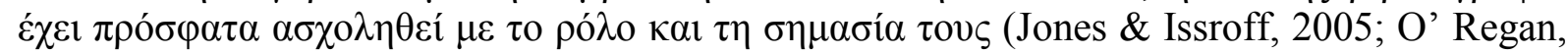

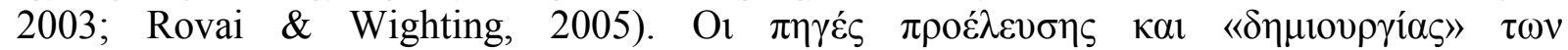

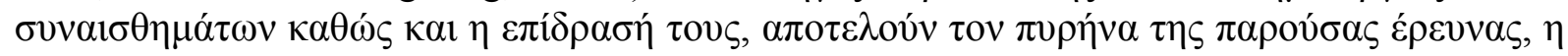

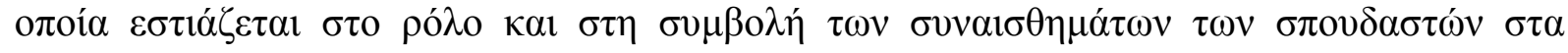

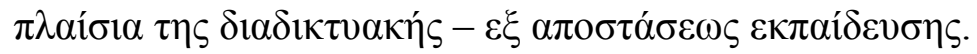

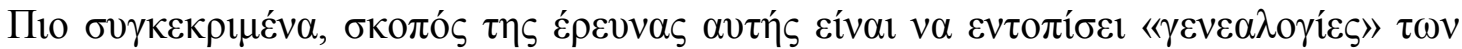

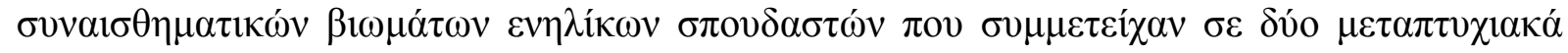

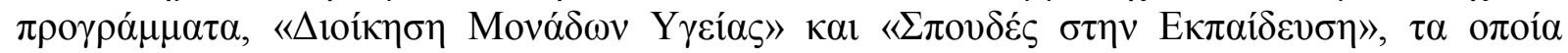

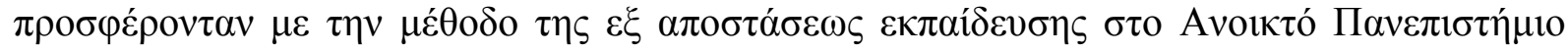

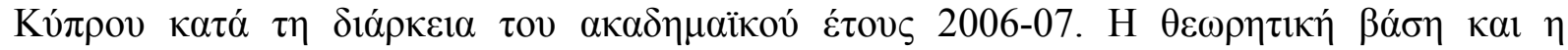

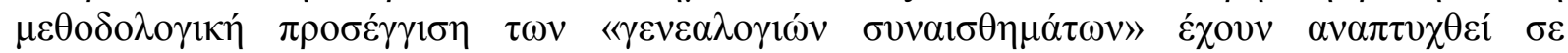

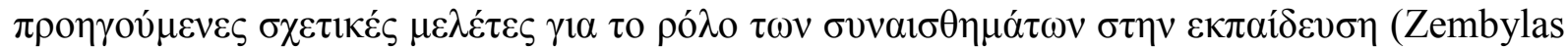

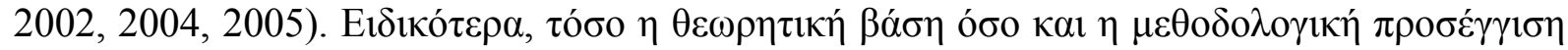

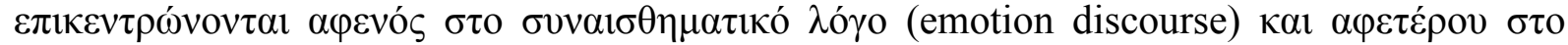

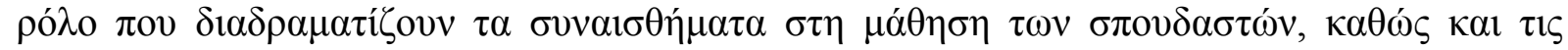

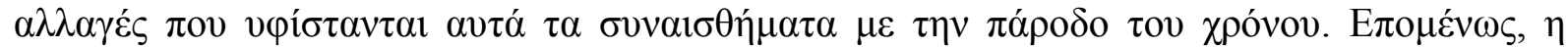

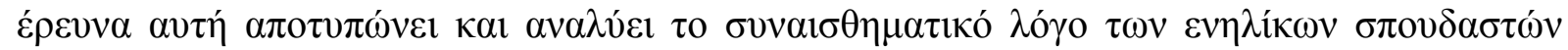

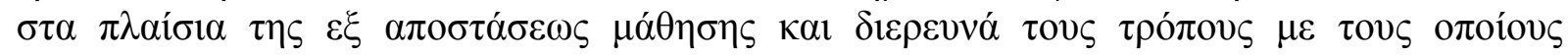

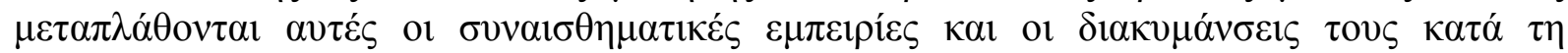

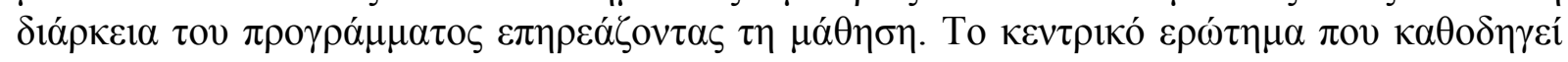

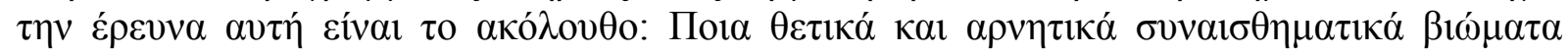

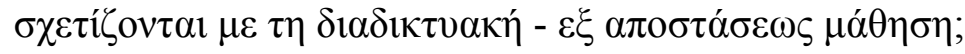

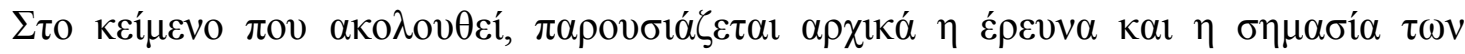

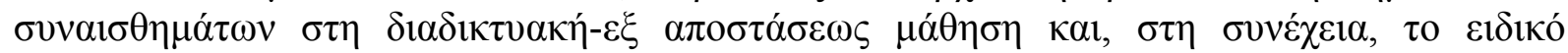

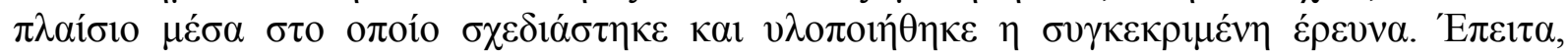

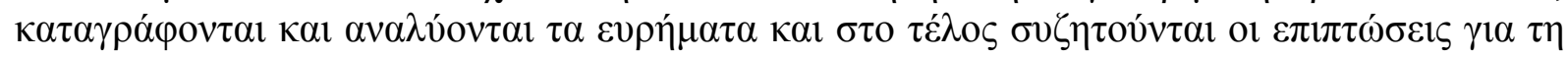

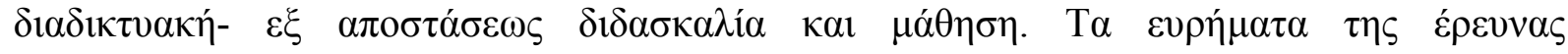

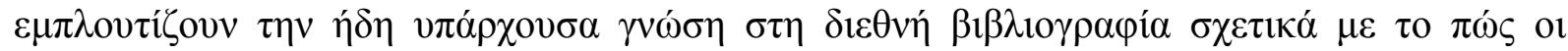

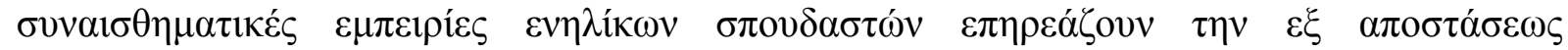

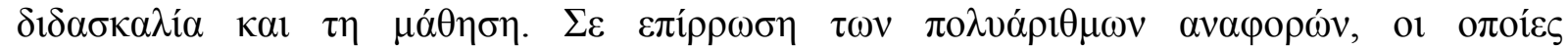

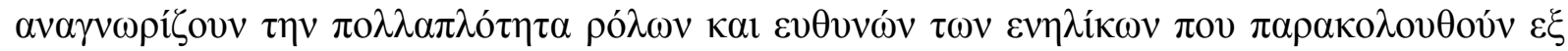

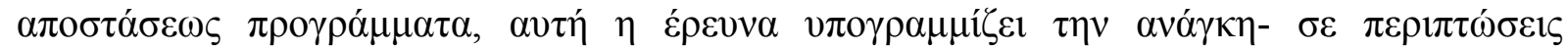

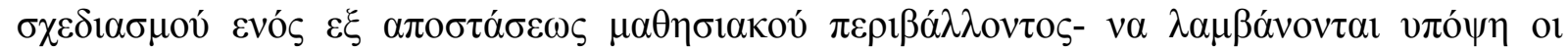

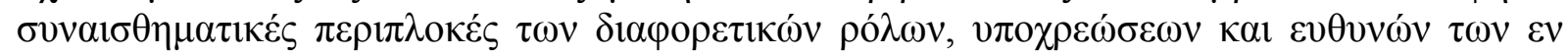

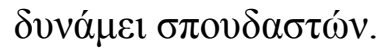

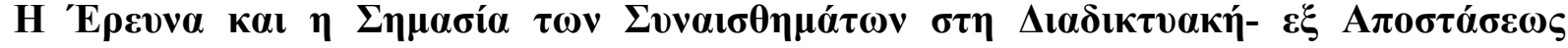 Мá $\theta \eta \sigma \eta$}




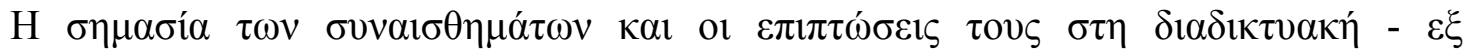

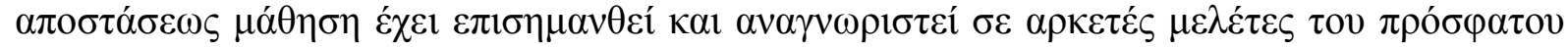

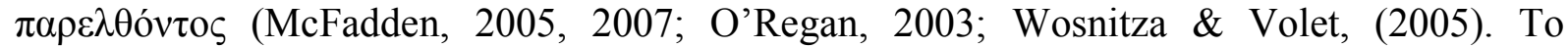

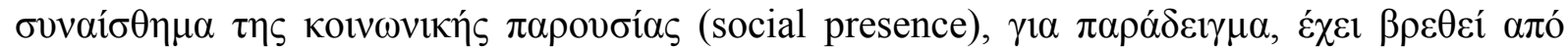

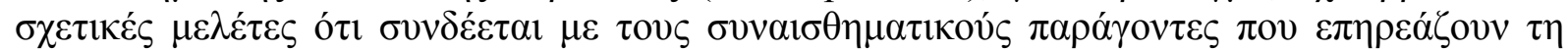

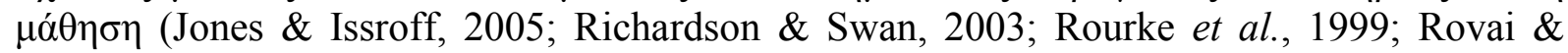

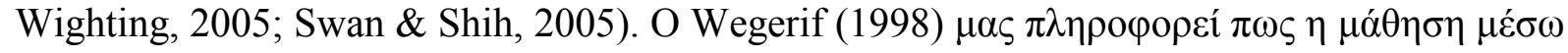

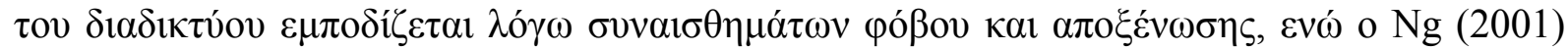

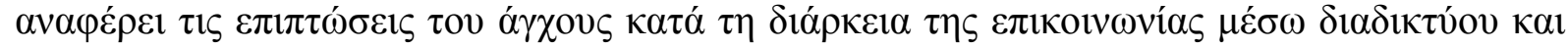

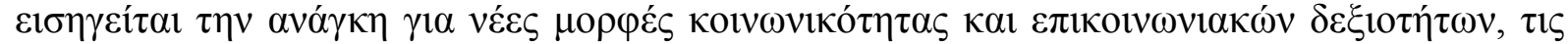

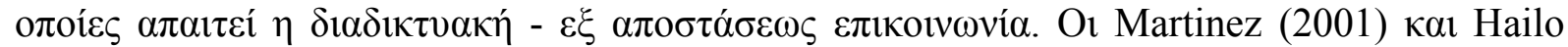

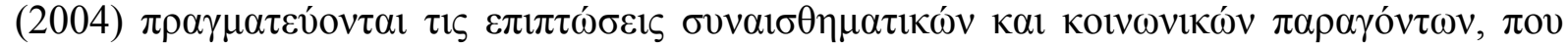

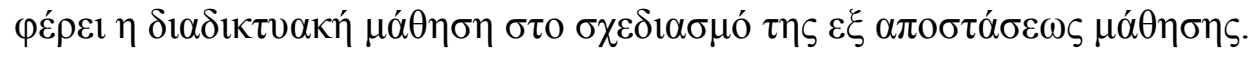

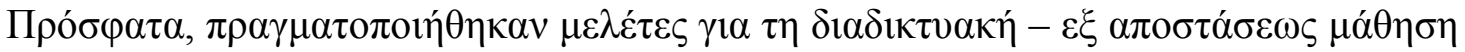

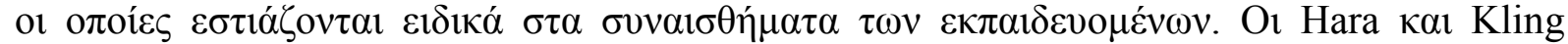

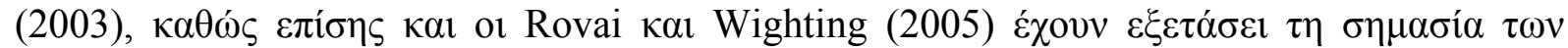

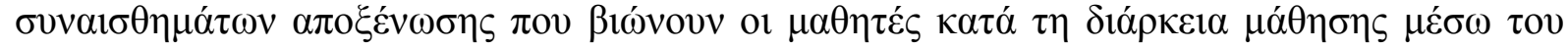

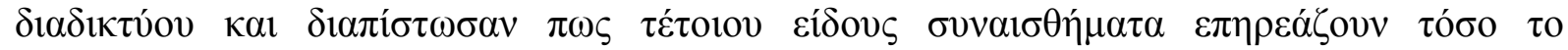

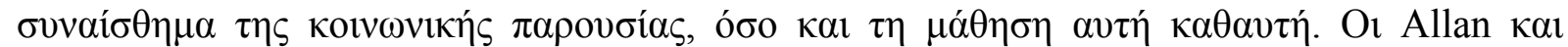

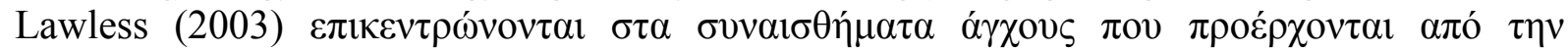

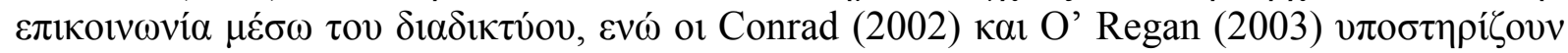

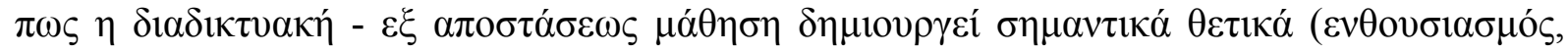

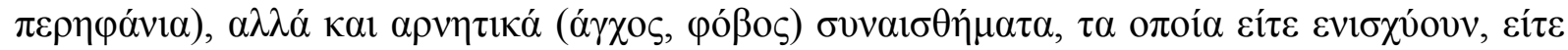

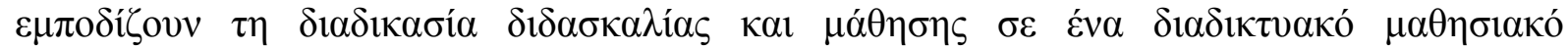

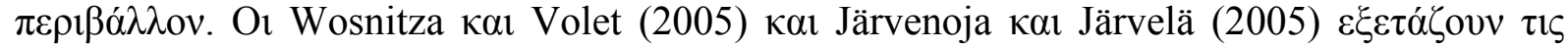

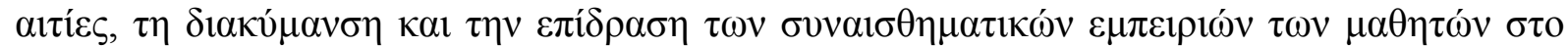

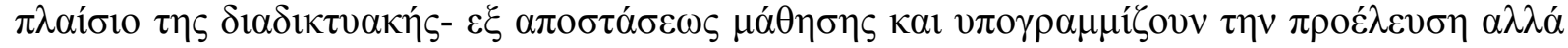

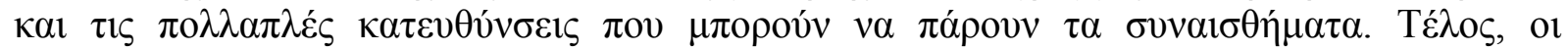

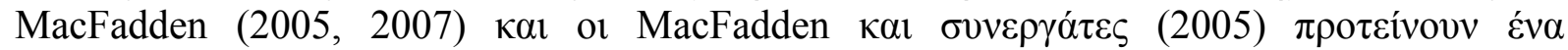

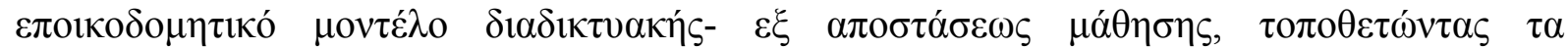

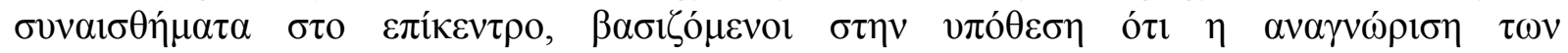

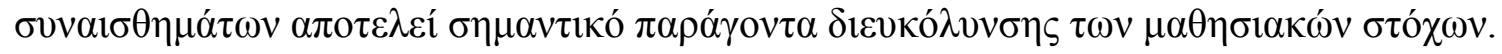

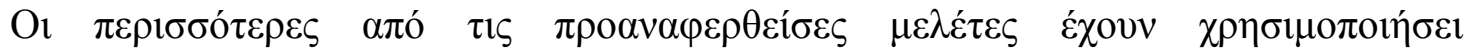

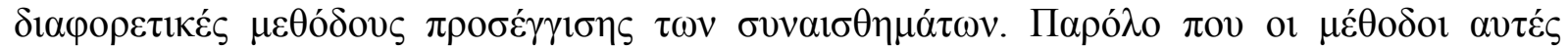

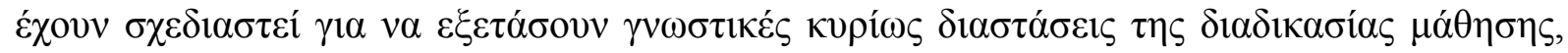

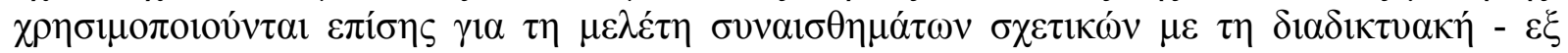

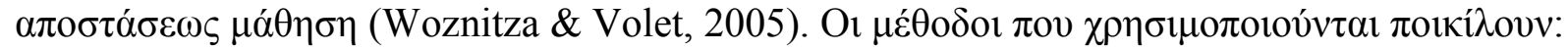

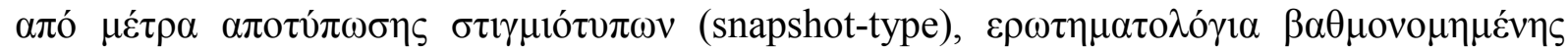

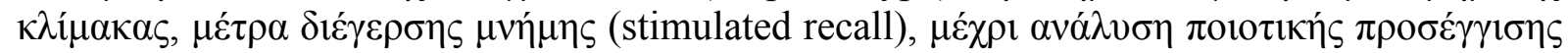

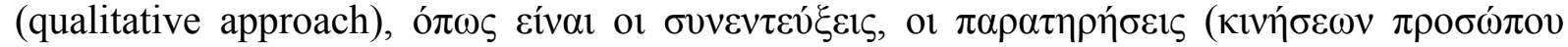

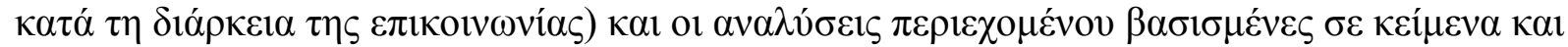

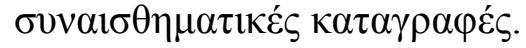

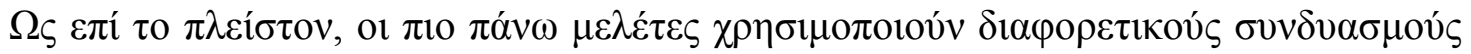

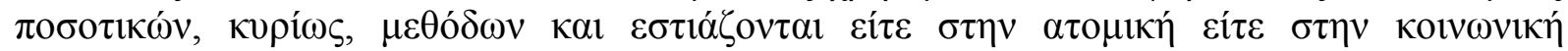

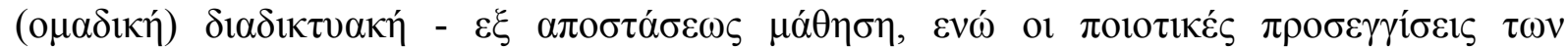

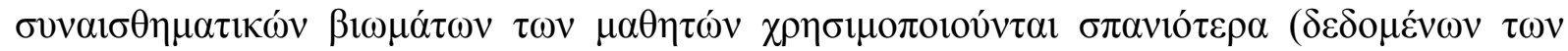

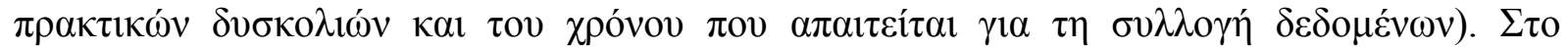

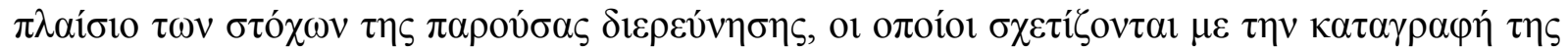




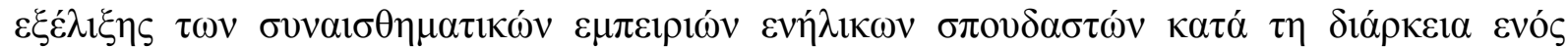

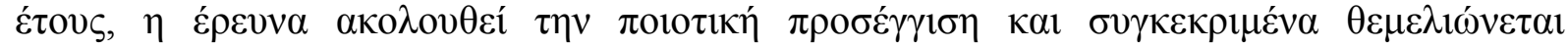

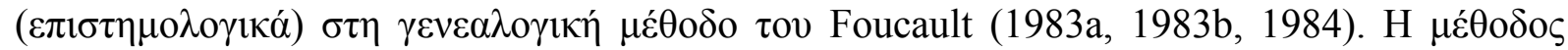

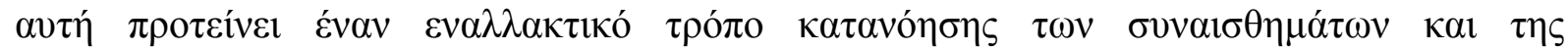

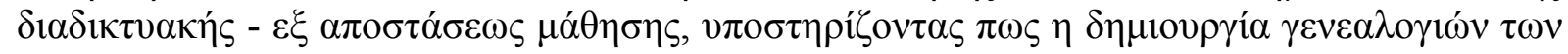

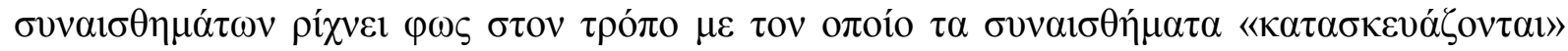

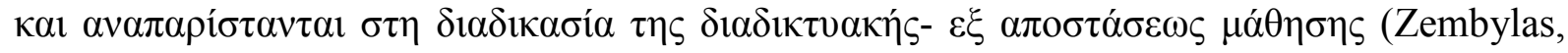
2002, 2004, 2005).

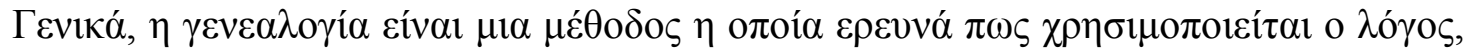

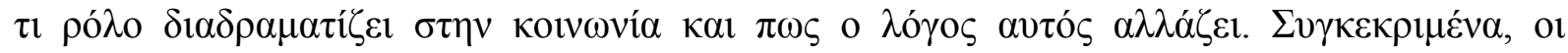

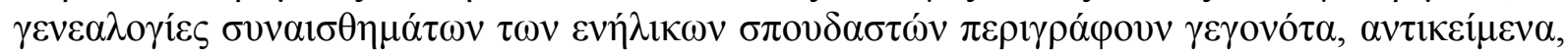

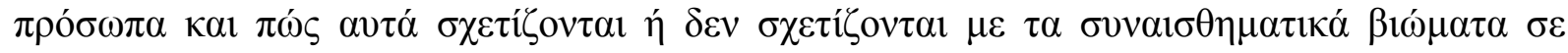

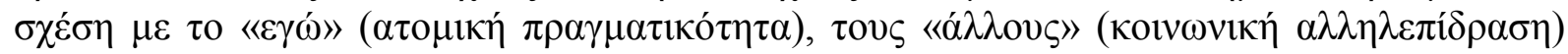

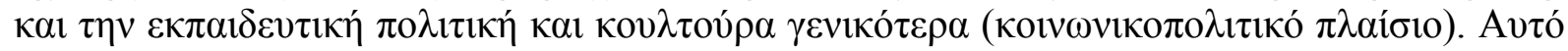

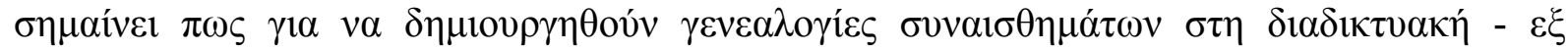

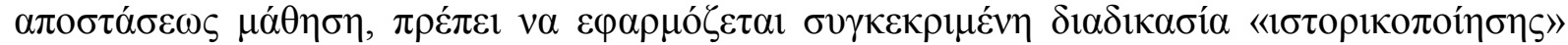

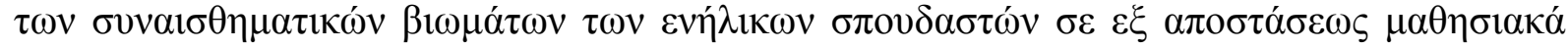

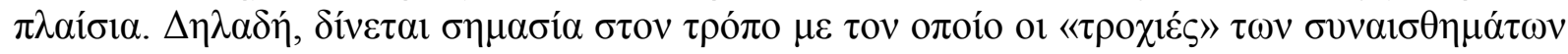

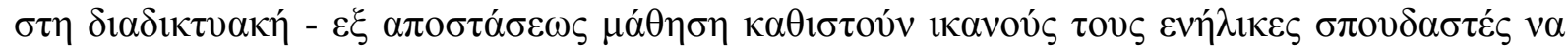

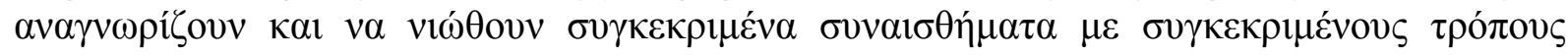
(Dirkx, 2001).

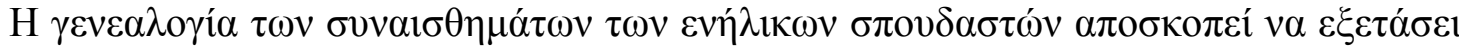

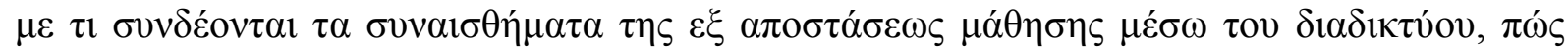

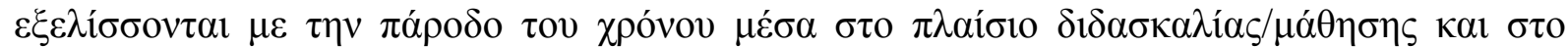

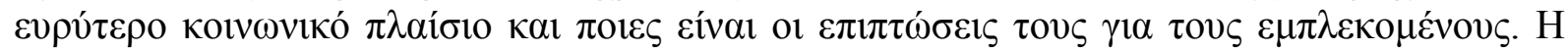

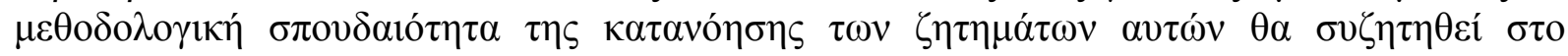
$\varepsilon \pi$ ó $\mu \varepsilon v o \mu \varepsilon \dot{\rho} \rho \varsigma$ $\tau \eta \varsigma \varepsilon \rho \gamma \alpha \sigma i ́ \alpha \varsigma$.

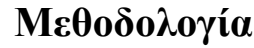

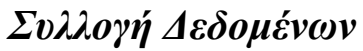

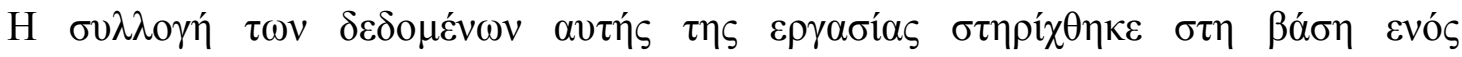

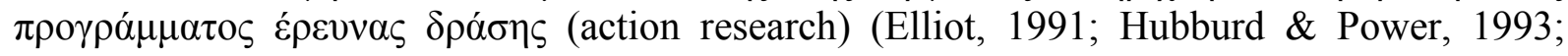

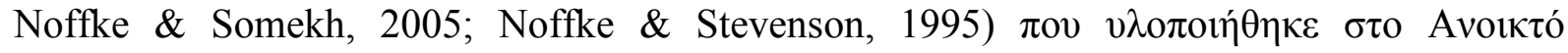

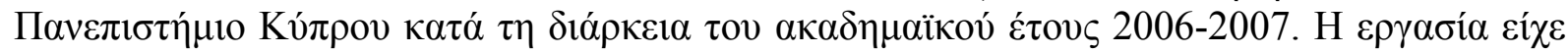

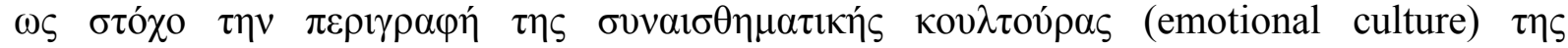

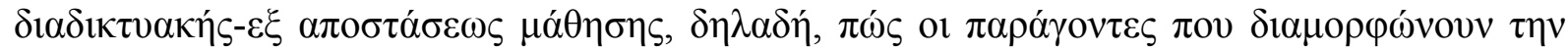

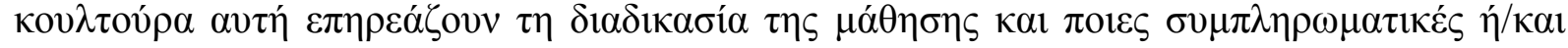

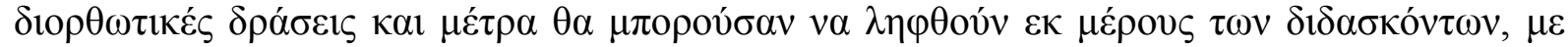

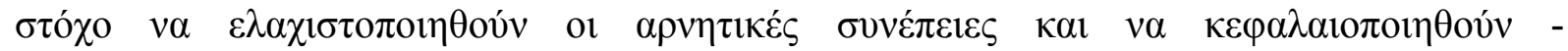

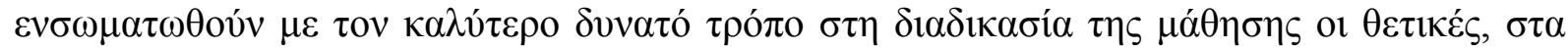

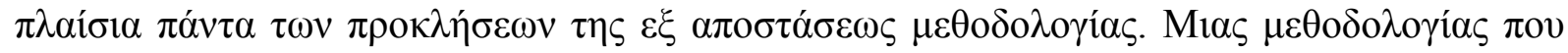

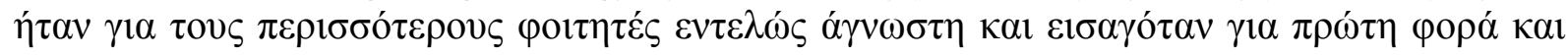

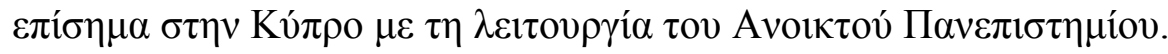

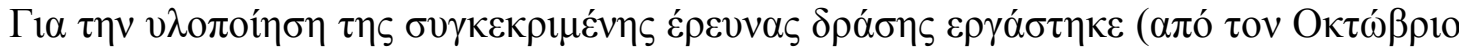

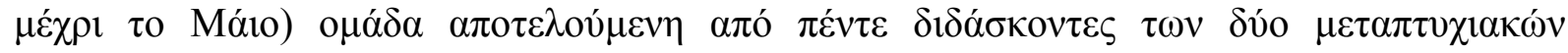

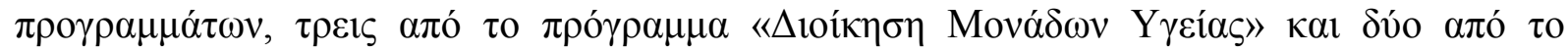

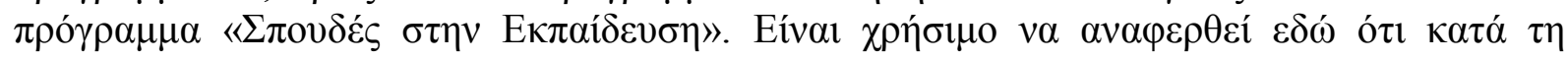




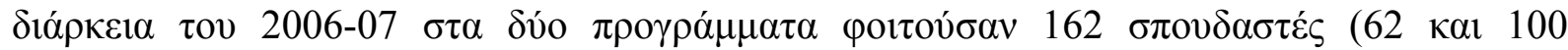

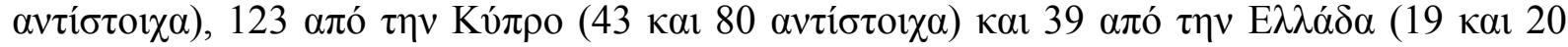

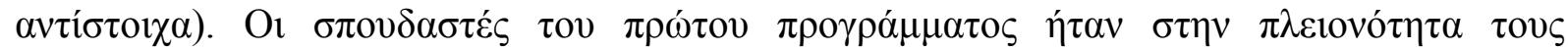

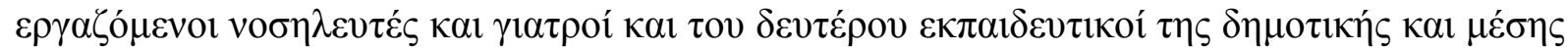

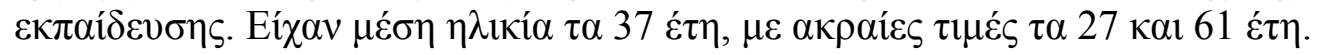

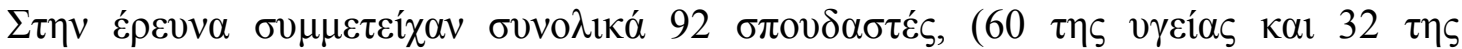

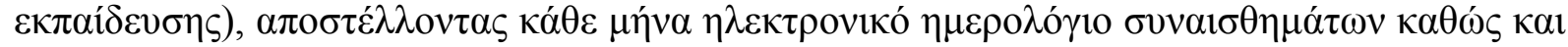

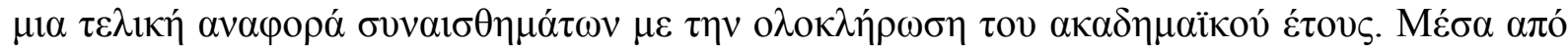

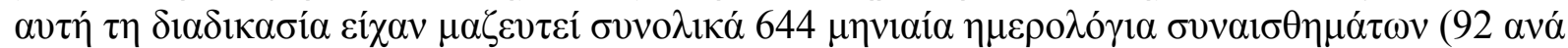

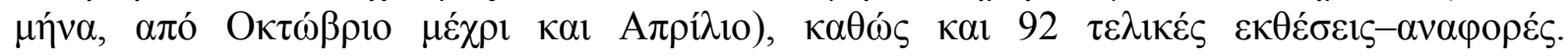

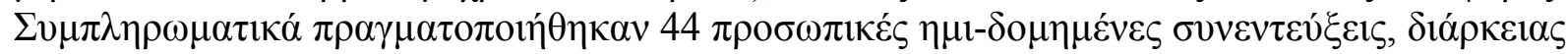

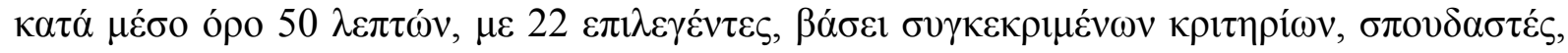

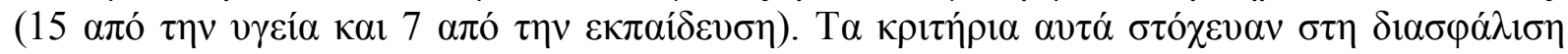

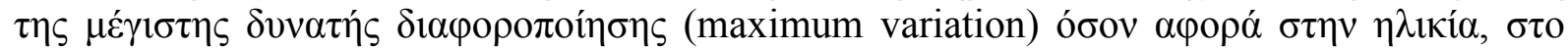

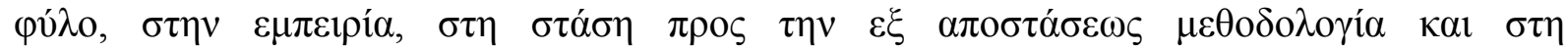

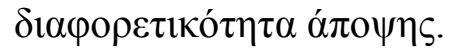

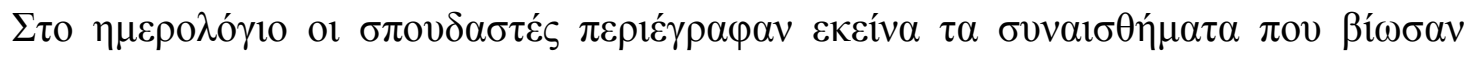

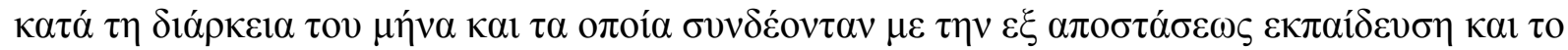

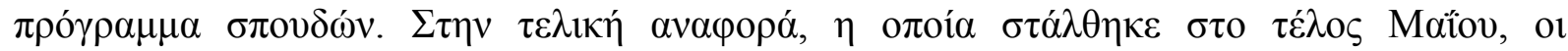

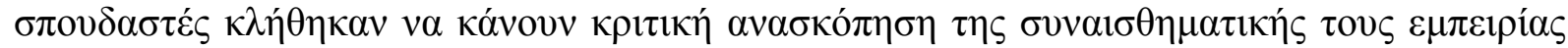

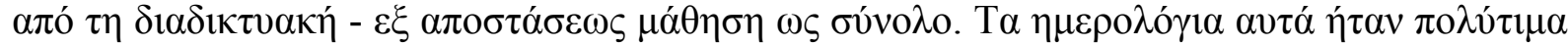

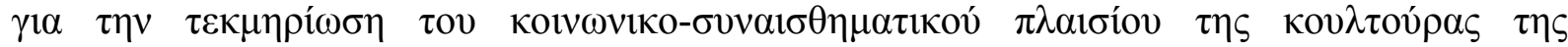

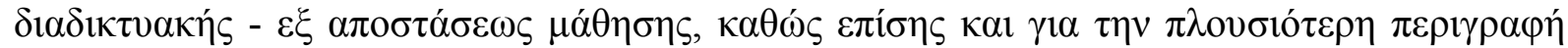

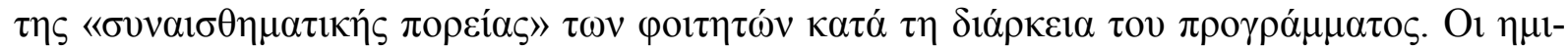

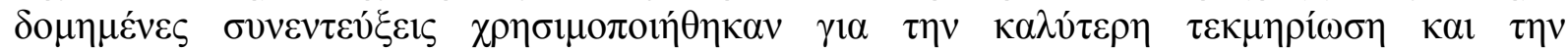

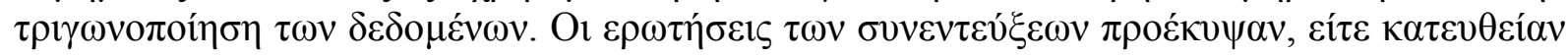

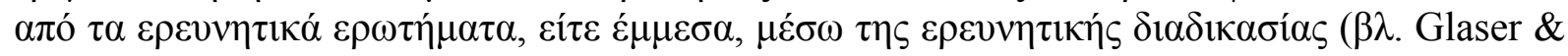

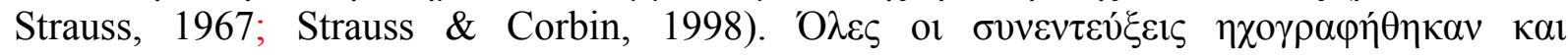
$\alpha \pi \circ \mu \alpha \gamma v \eta \tau о \varphi \omega v \eta ́ \theta \eta \kappa \alpha v$.

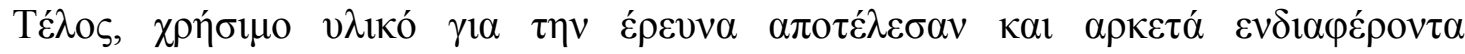

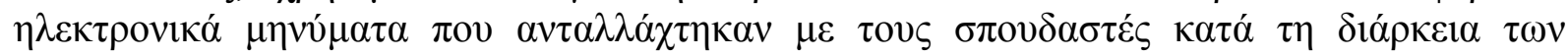

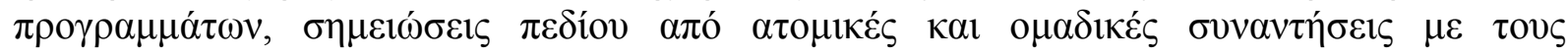

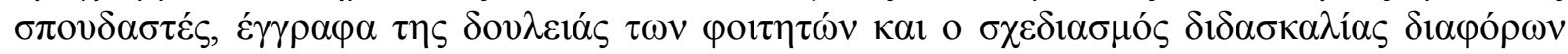
$\pi \tau v \chi \omega ́ v \tau o v \mu \alpha \theta \eta ́ \mu \alpha \tau o \varsigma$.

\section{$A v a ́ \lambda v \sigma \eta \delta \varepsilon \delta o \mu \varepsilon ́ v \omega v$}

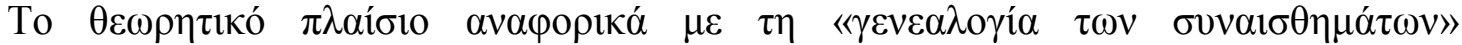

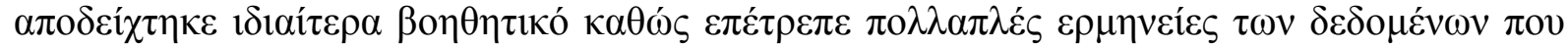

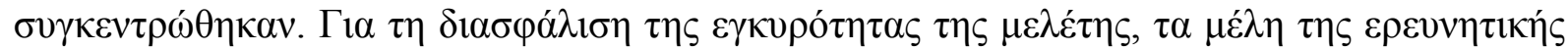

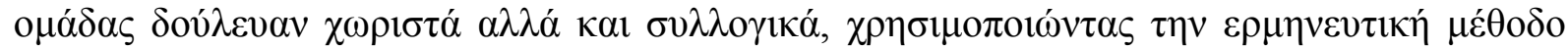

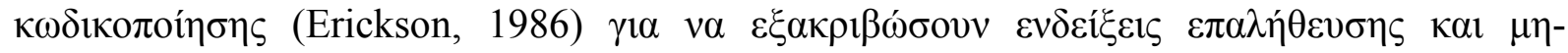

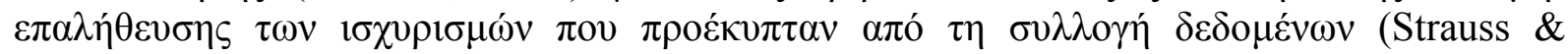

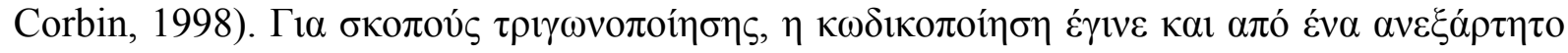

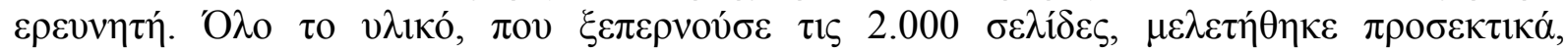

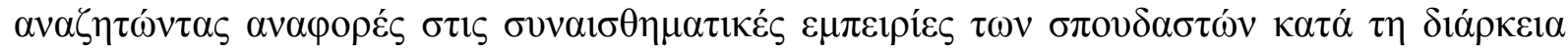

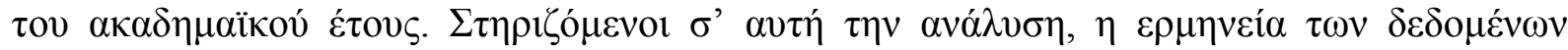

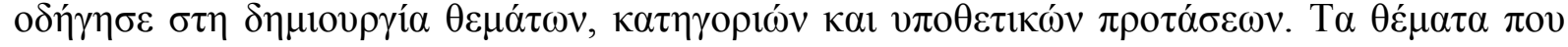




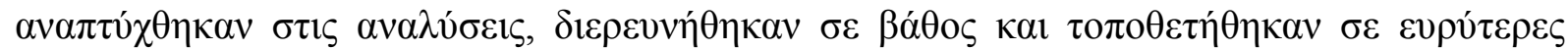

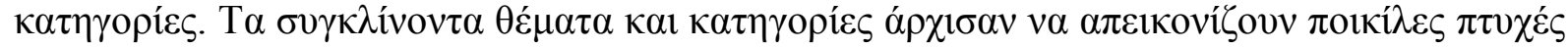

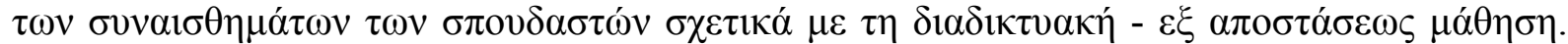

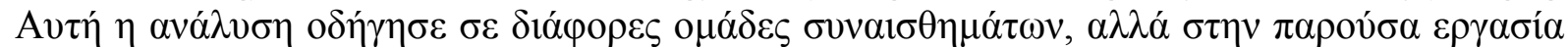

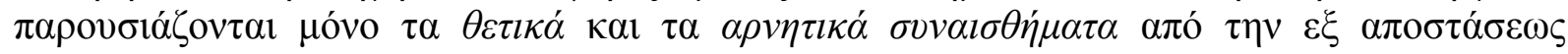

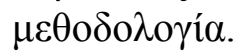

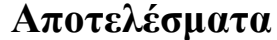

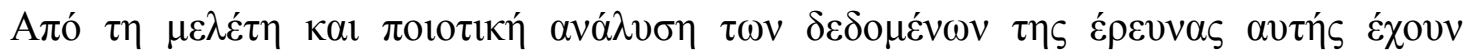

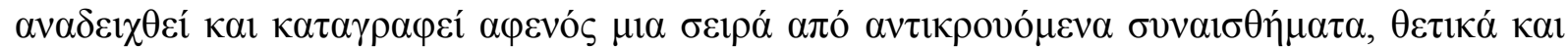

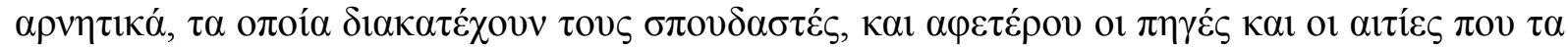

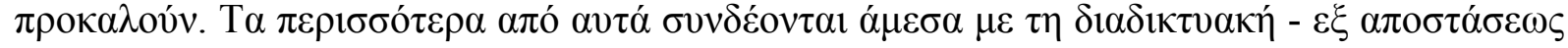

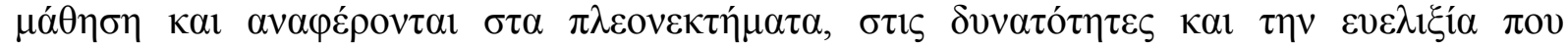

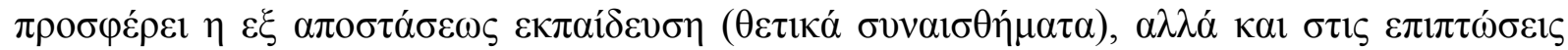

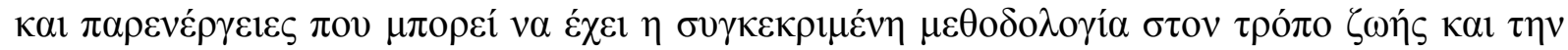

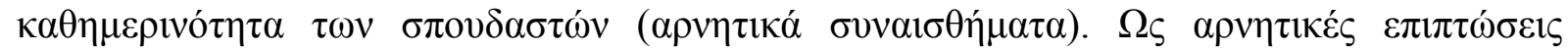

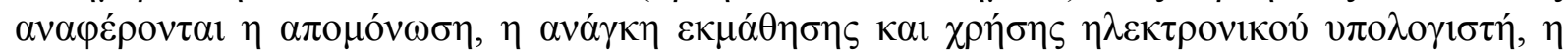

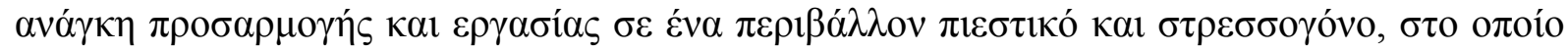

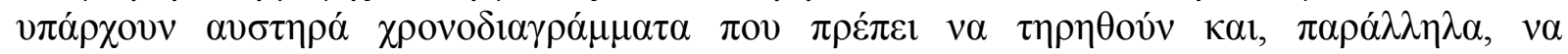

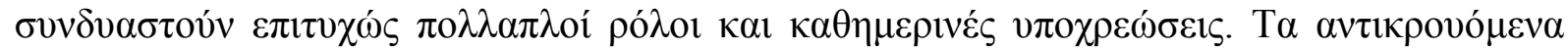

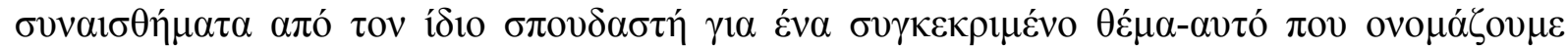

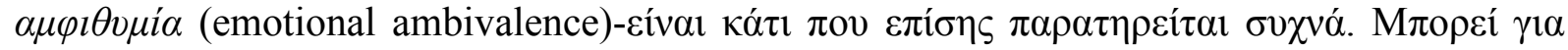

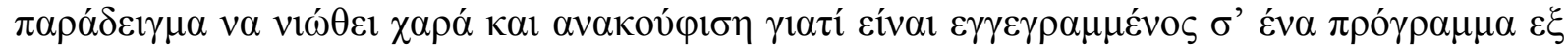

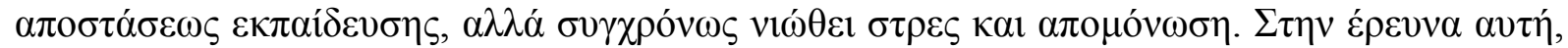

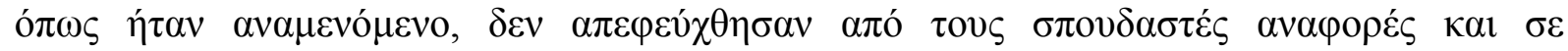

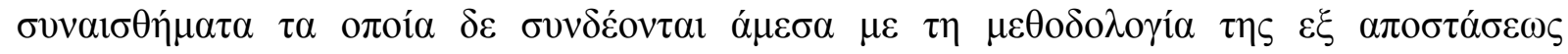

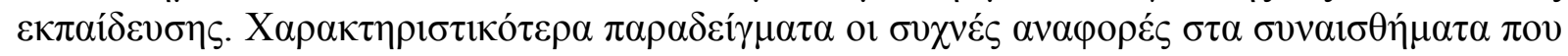

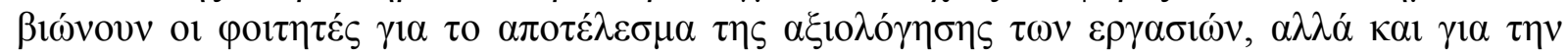

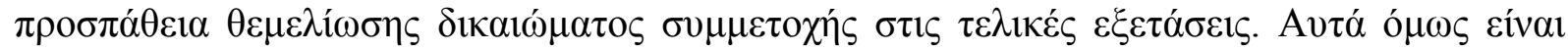

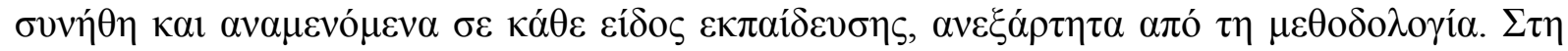

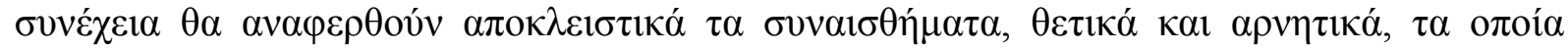

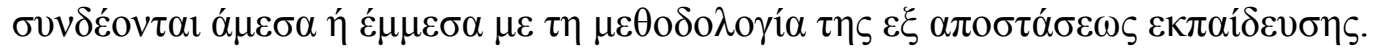

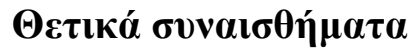

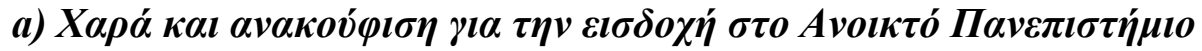

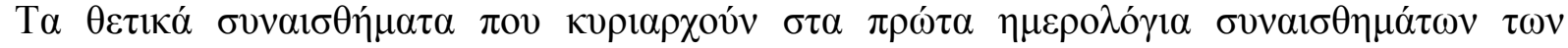

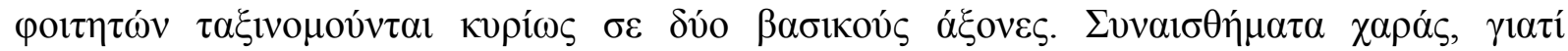

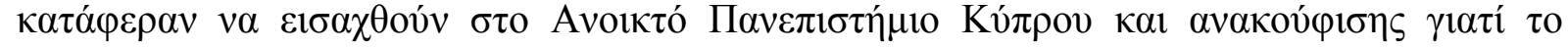

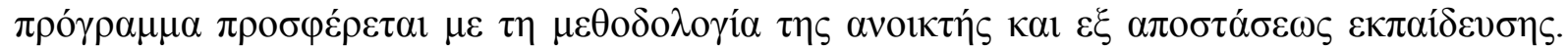

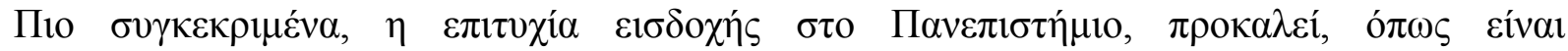

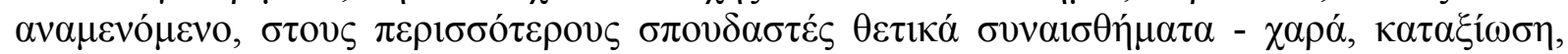

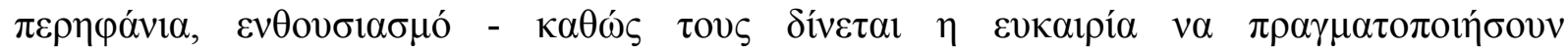

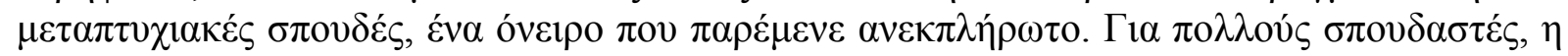

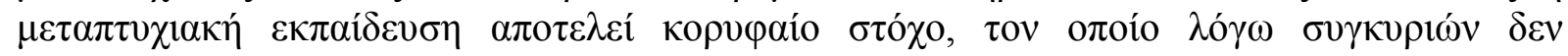

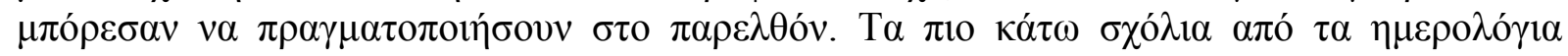

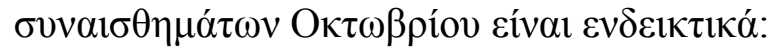




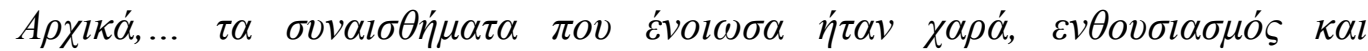

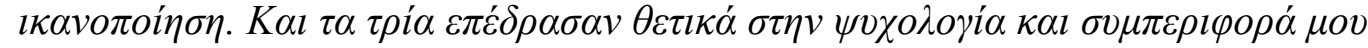

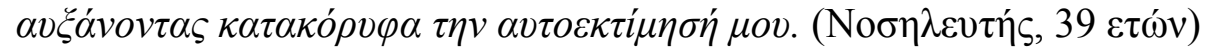

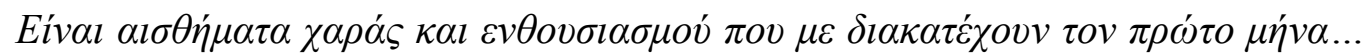

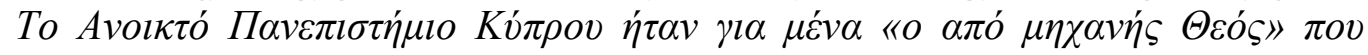

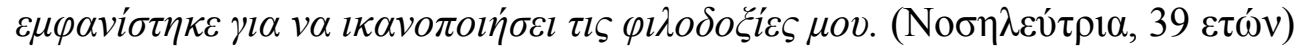

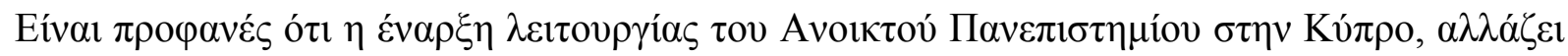

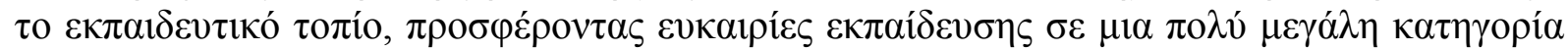

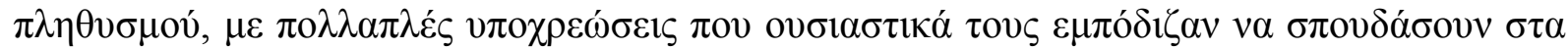

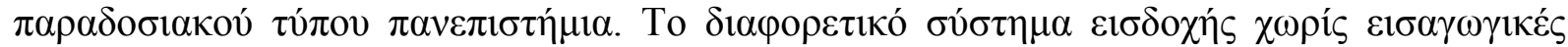

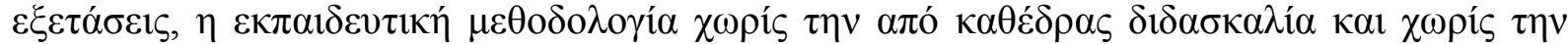

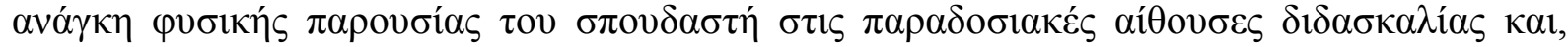

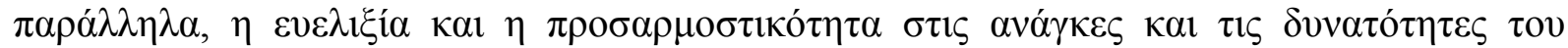

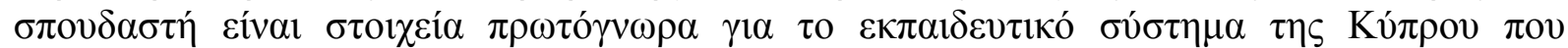

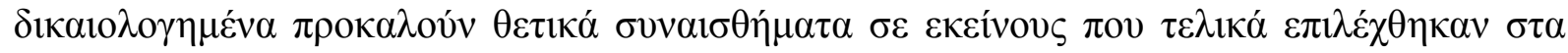

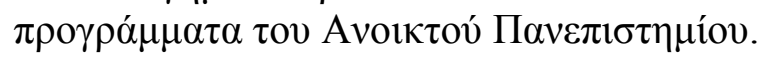

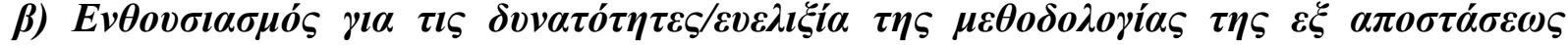 $\varepsilon \kappa \pi \alpha i ́ \delta \varepsilon v \sigma \eta \varsigma$}

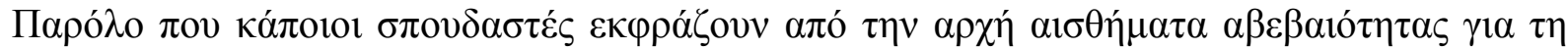

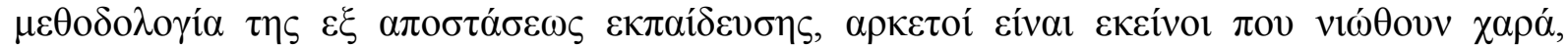

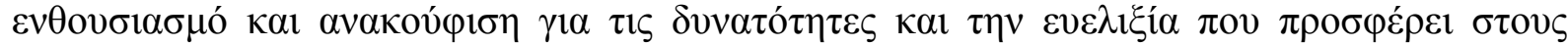

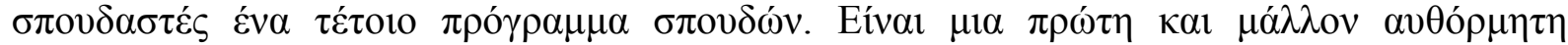

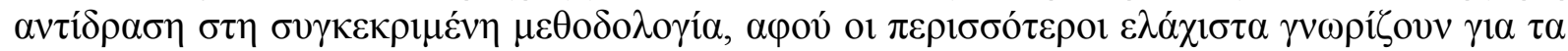

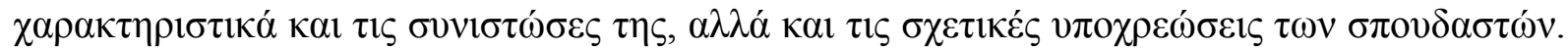

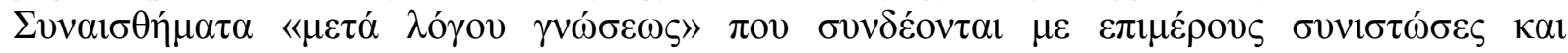

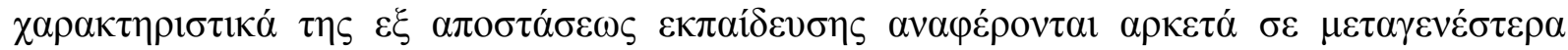

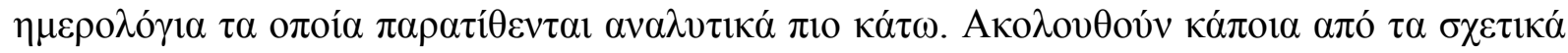

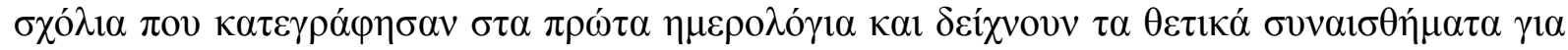

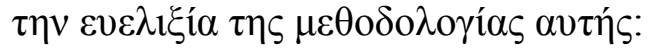

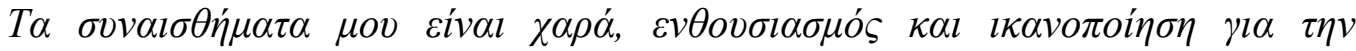

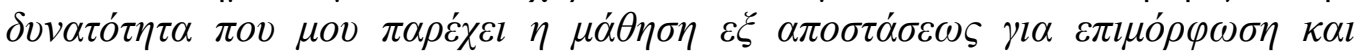

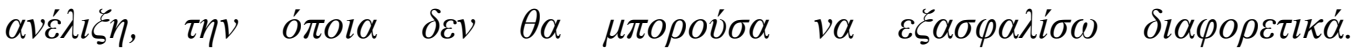

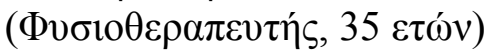

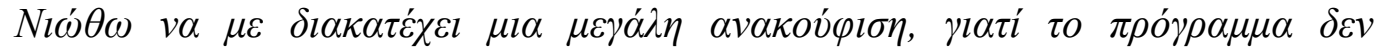

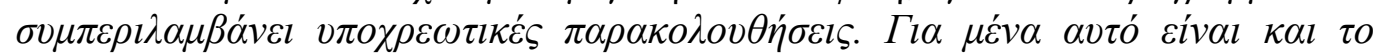

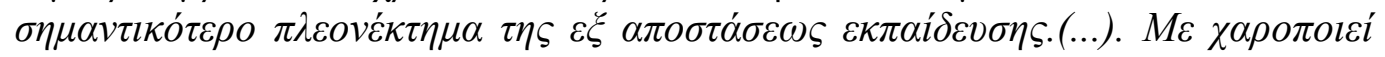

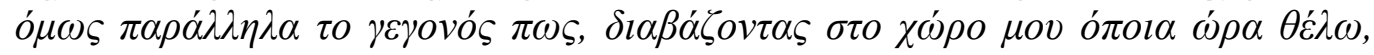

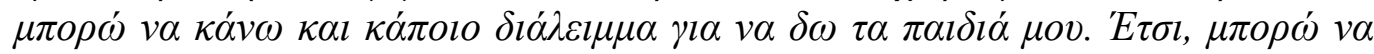

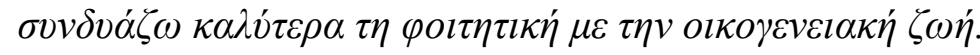

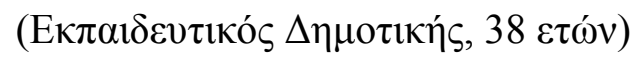

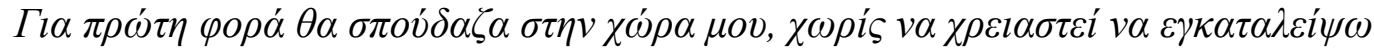

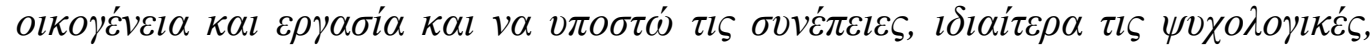

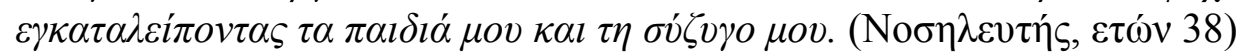




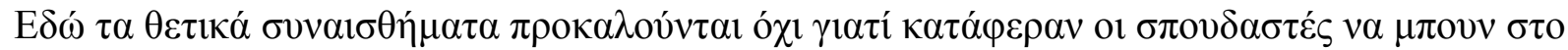

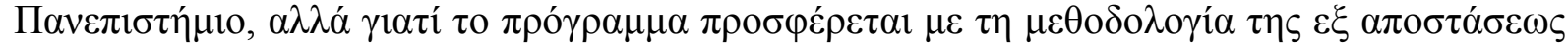

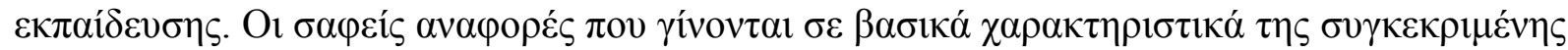

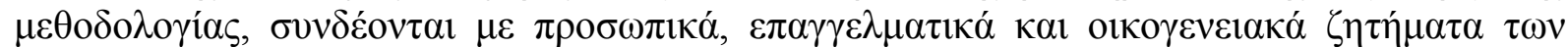

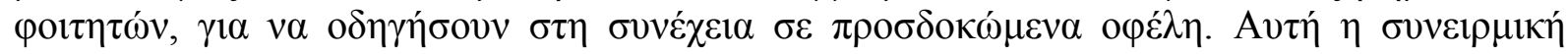

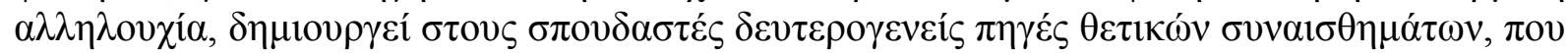

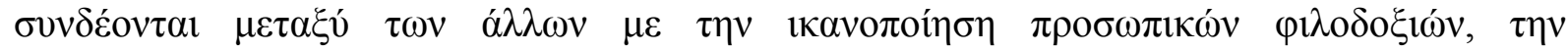

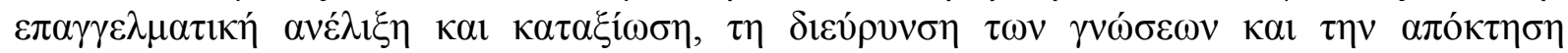
$\pi \rho 0 \sigma o ́ v \tau \omega v$.

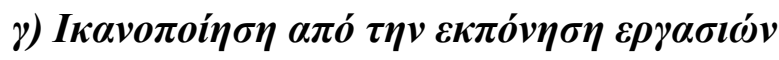

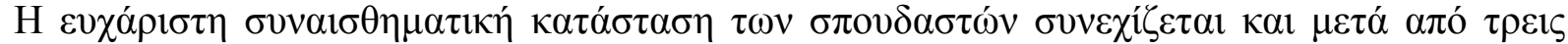

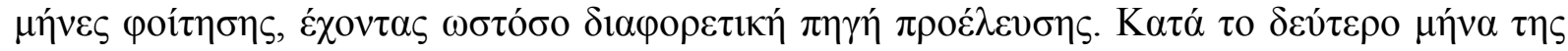

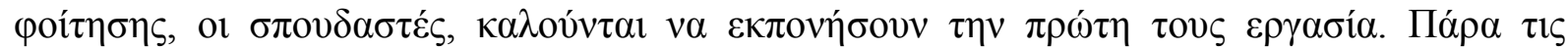

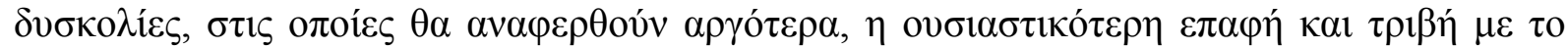

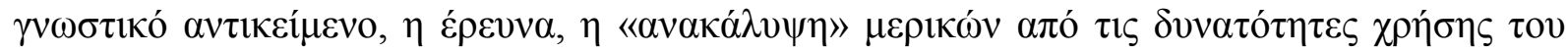

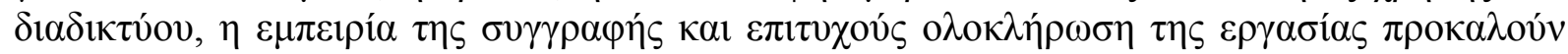

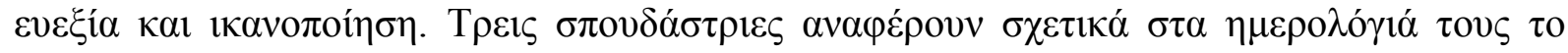
$\mu \eta \dot{v} \alpha$ Noв́ $\mu \beta \rho ı$.

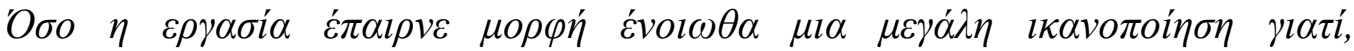

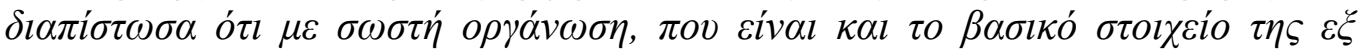

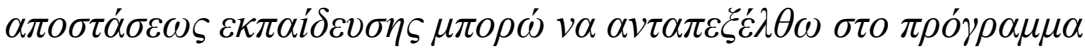

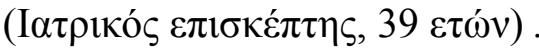

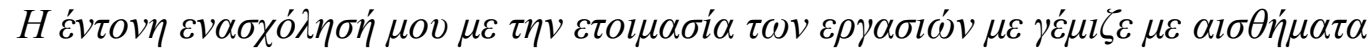

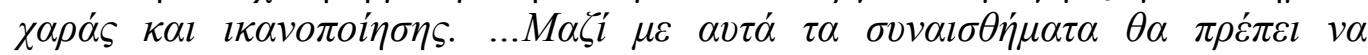

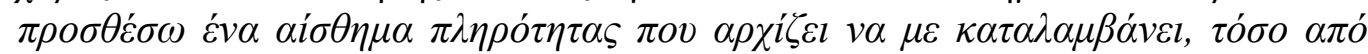

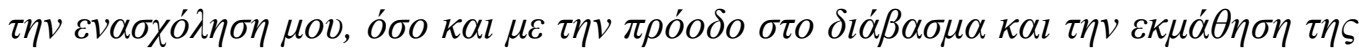
vं$\lambda \eta \varsigma$

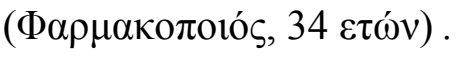

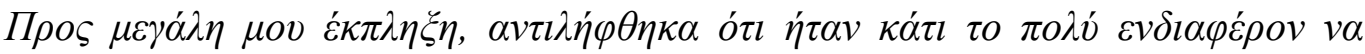

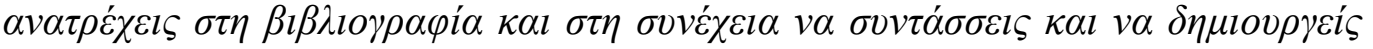

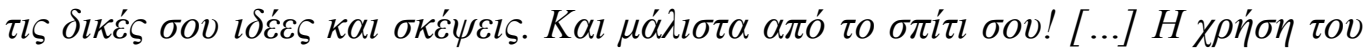
$\delta \imath \alpha \delta \imath \kappa \tau \dot{o v} \quad \sigma \tau \eta \quad \mu \alpha ́ \theta \eta \sigma \eta \quad \pi \alpha \rho \varepsilon_{\chi} \varepsilon l$

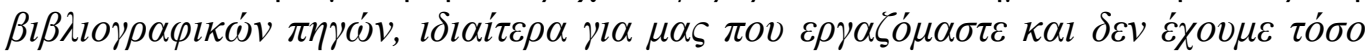

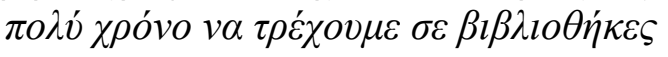

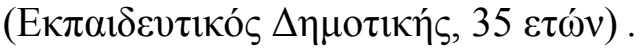

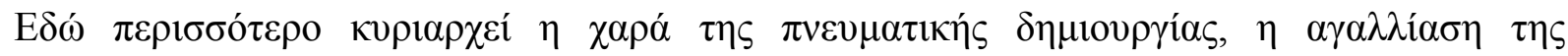

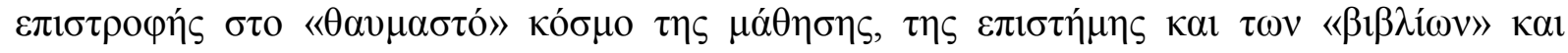

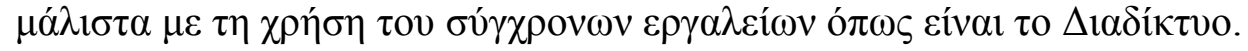




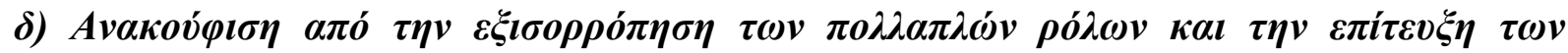 $\sigma \tau \dot{\alpha} \chi \omega v$}

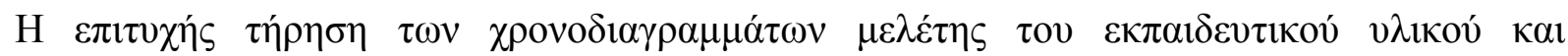

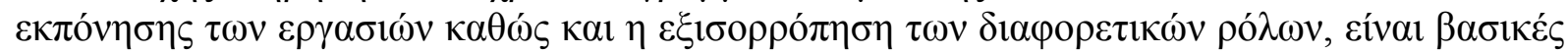

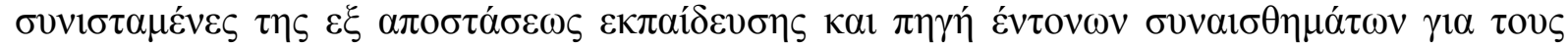

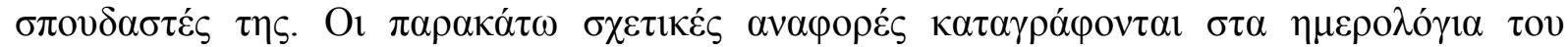

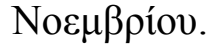

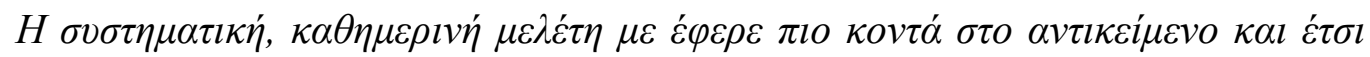

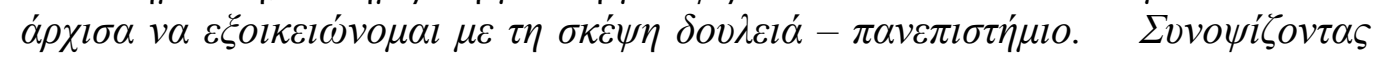

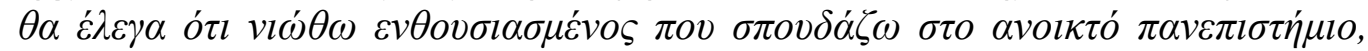

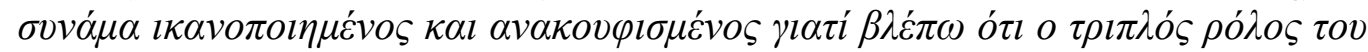

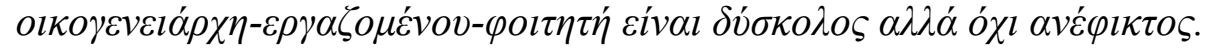

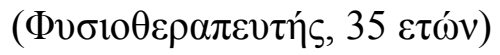

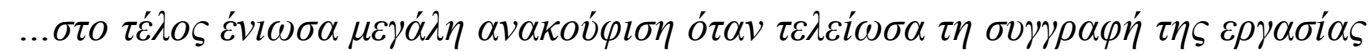

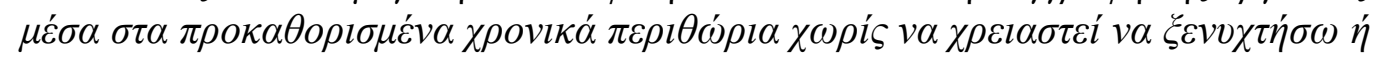

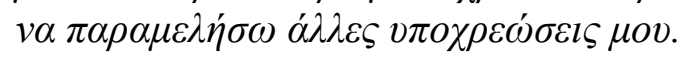

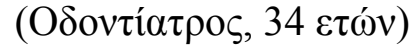

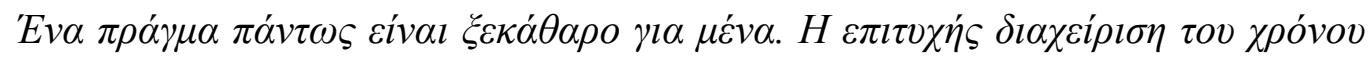

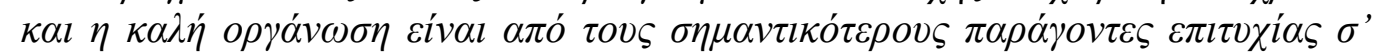

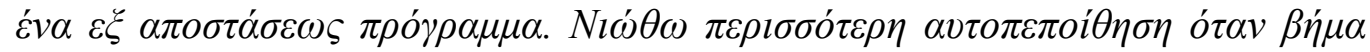

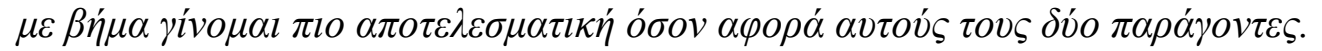

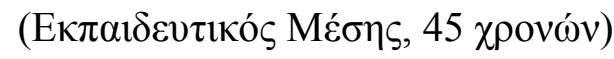

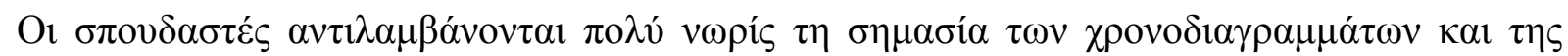

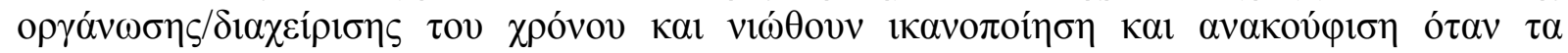

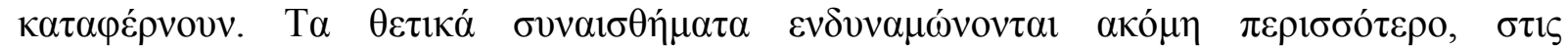

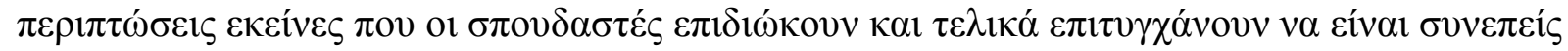

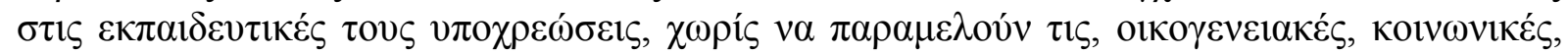

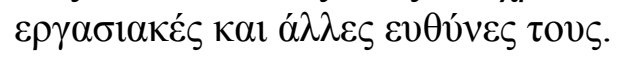

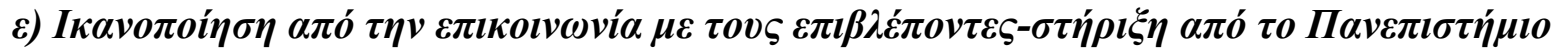

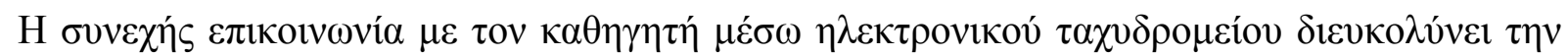

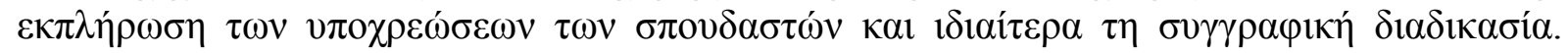

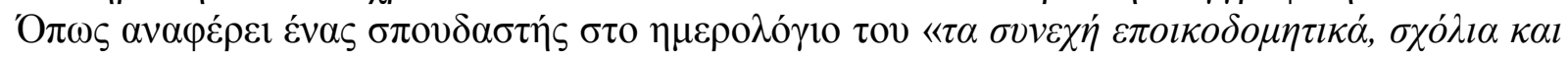

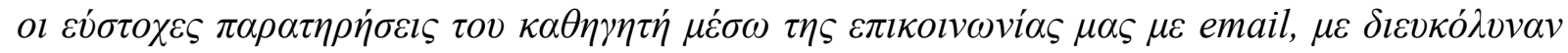

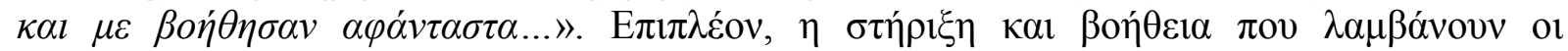

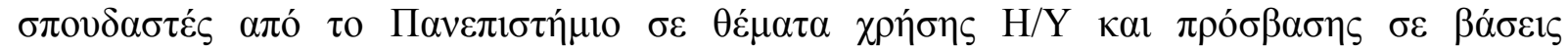

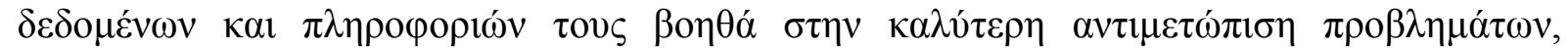

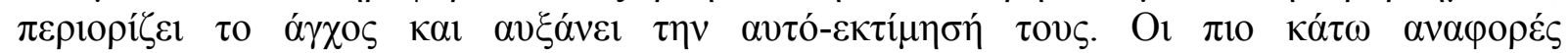

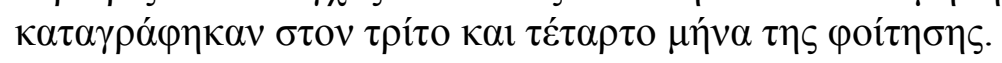




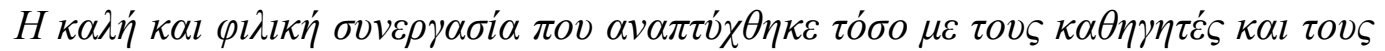

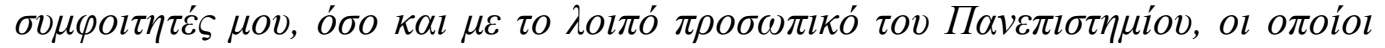

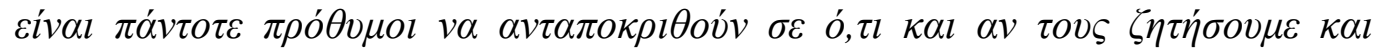

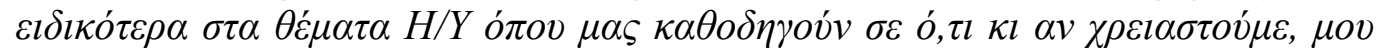

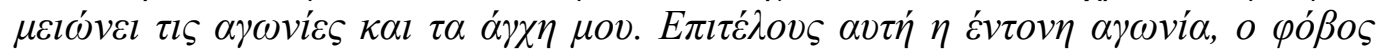

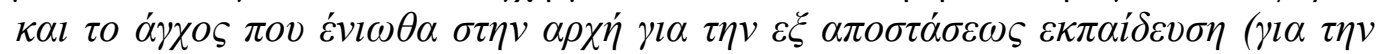

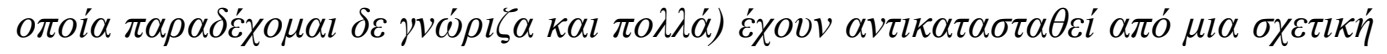

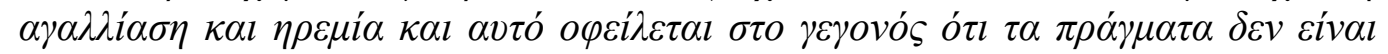

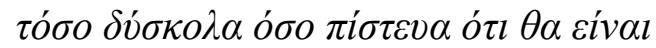

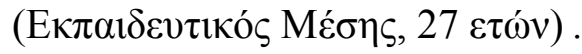

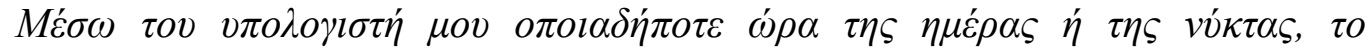

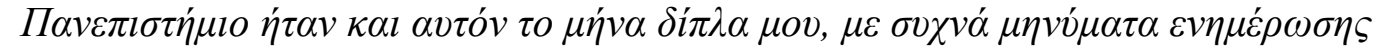

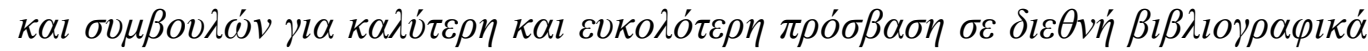

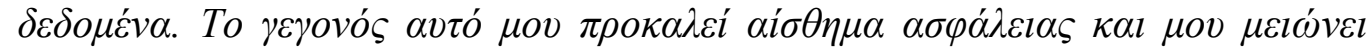

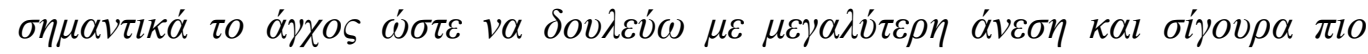
$\alpha \pi \circ \delta o \tau \iota \kappa \alpha ́$.

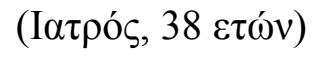

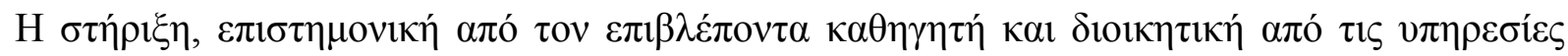

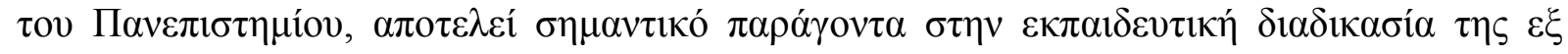

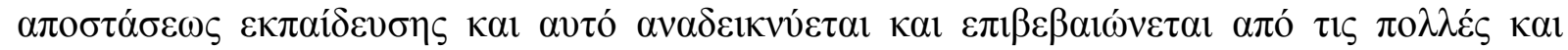

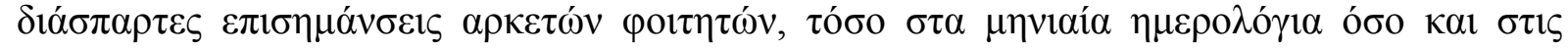

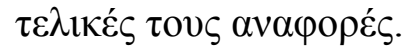

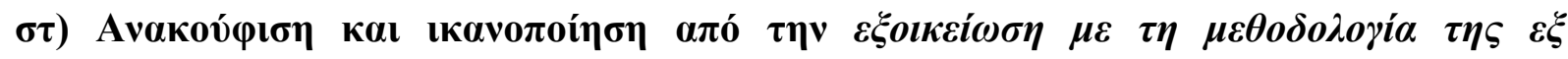 $\alpha \pi 0 \sigma \tau a ́ \sigma \varepsilon \omega \varsigma \varepsilon \kappa \pi \alpha i ́ \delta \varepsilon v \sigma \eta \kappa \alpha \imath \tau \eta \chi \rho \eta ́ \sigma \eta \tau o v ~ H / Y$}

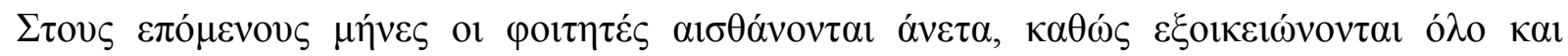

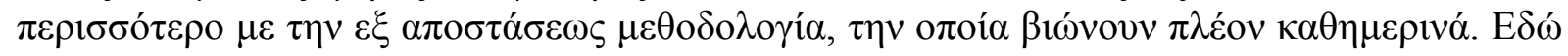

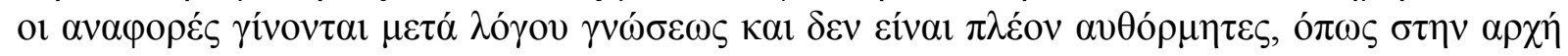

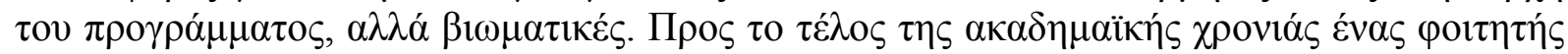

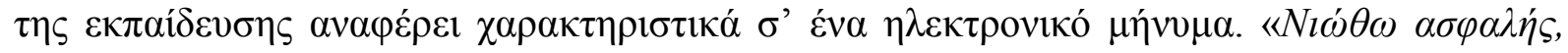

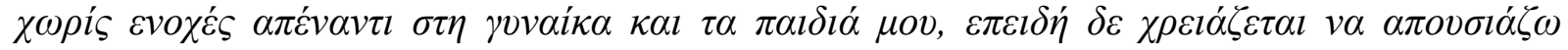

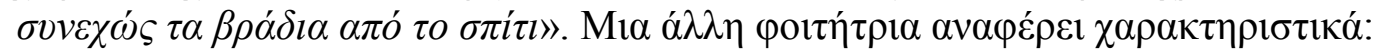

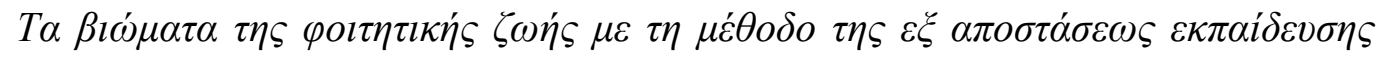

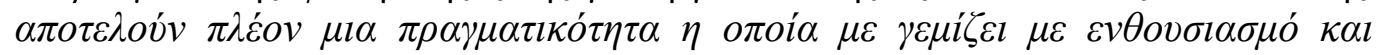

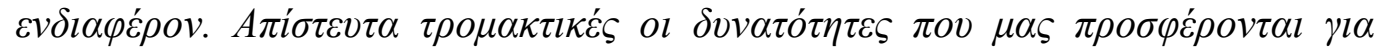

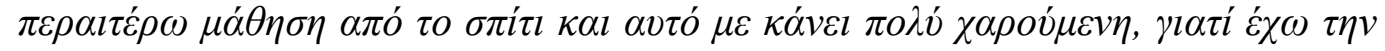

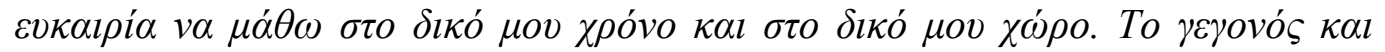

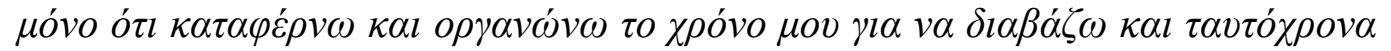

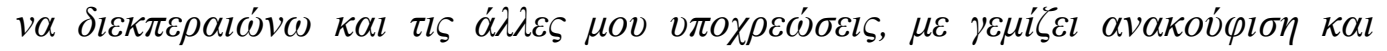

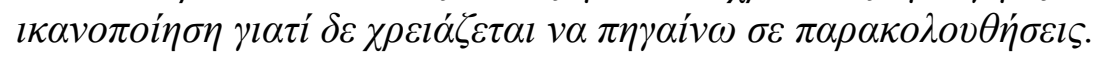

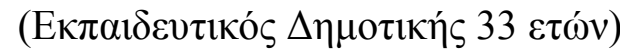

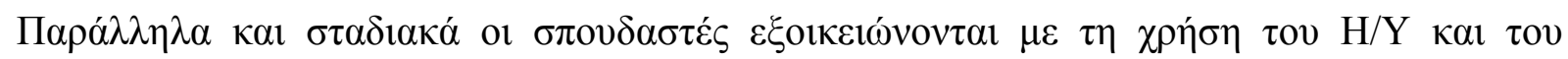

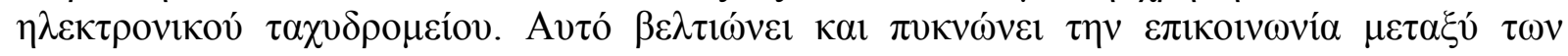

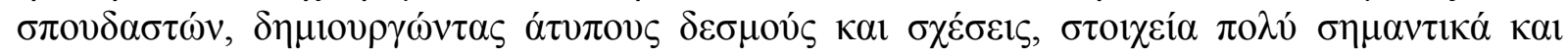

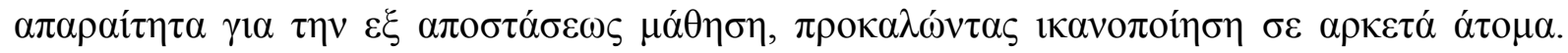




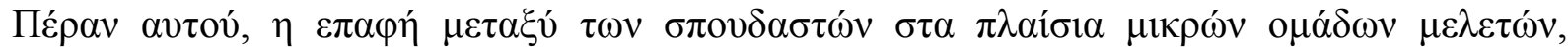

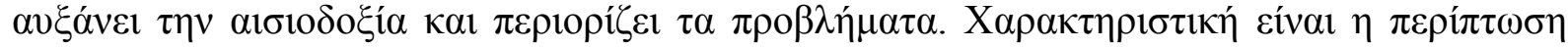

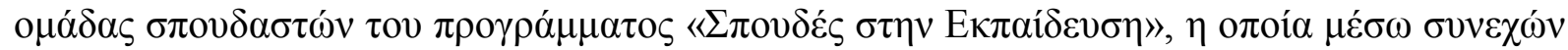

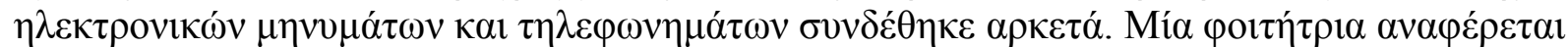

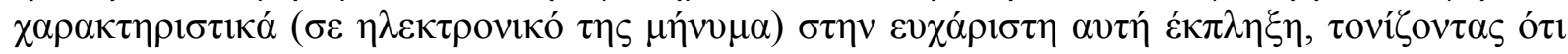

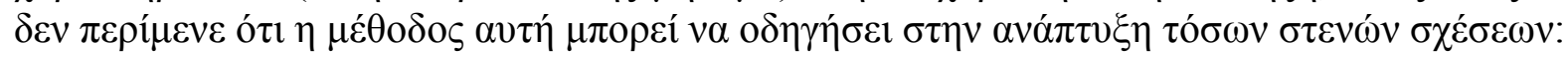

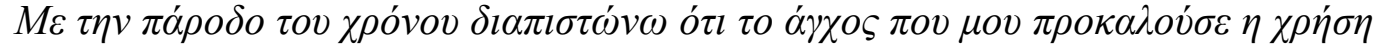

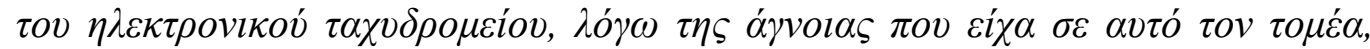

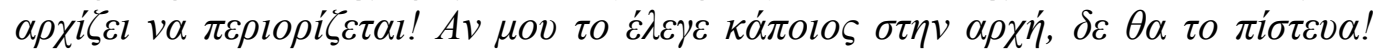

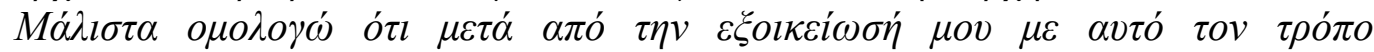

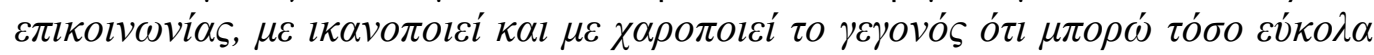

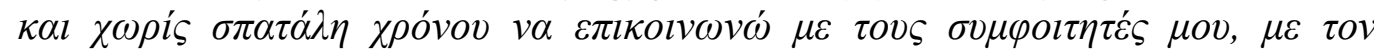

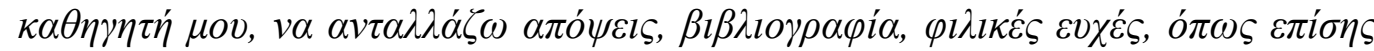

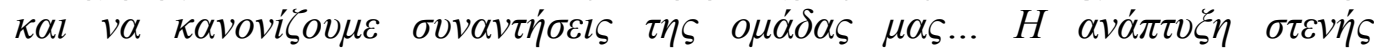

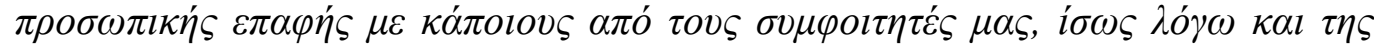

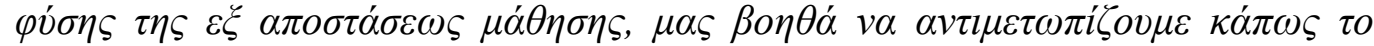

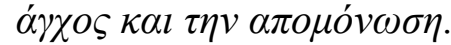

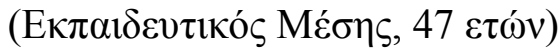

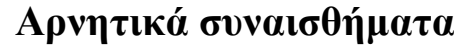

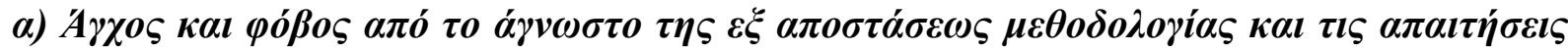

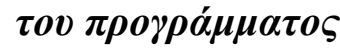

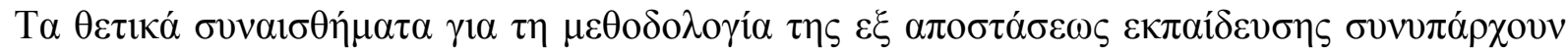

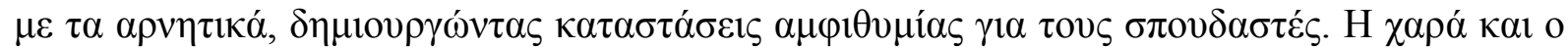

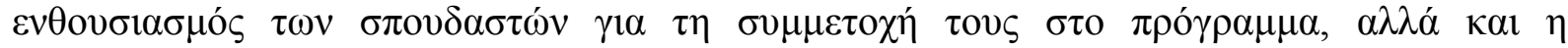

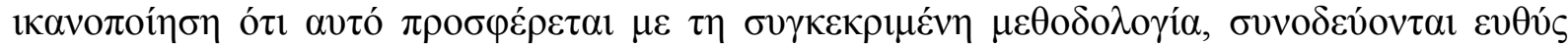

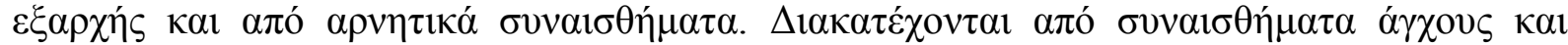

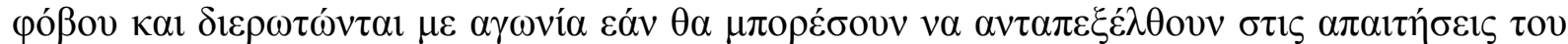

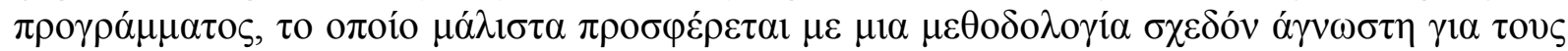

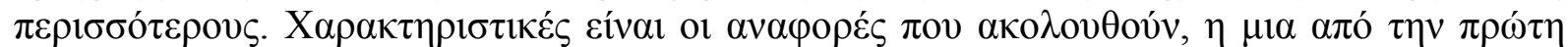

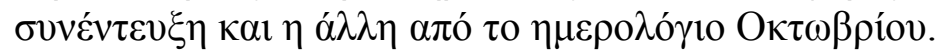

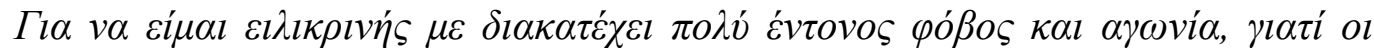

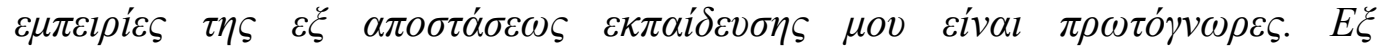

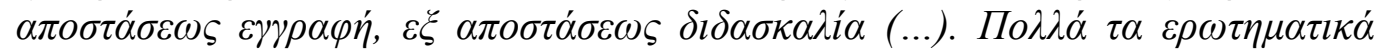

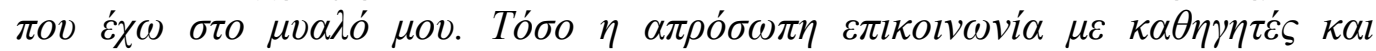

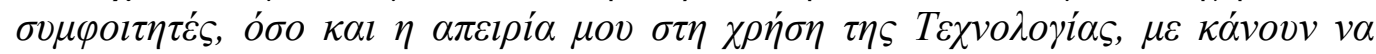

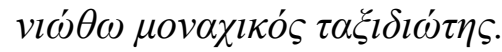

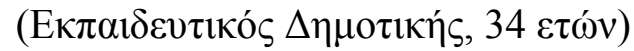

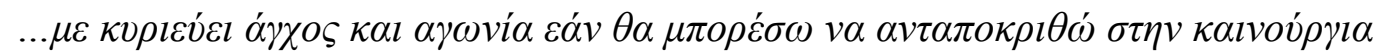

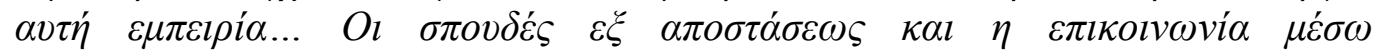

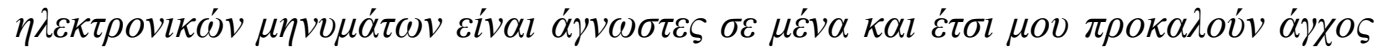

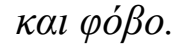

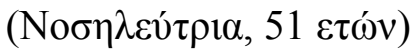




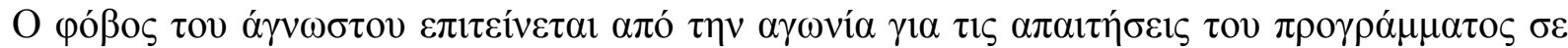

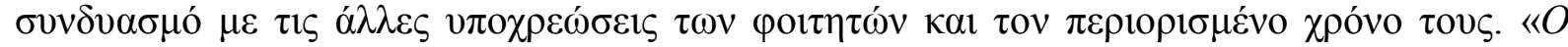

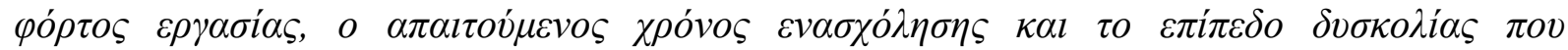

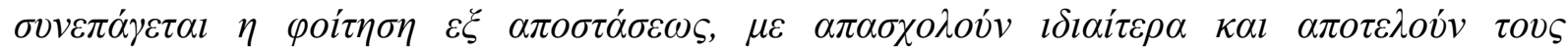

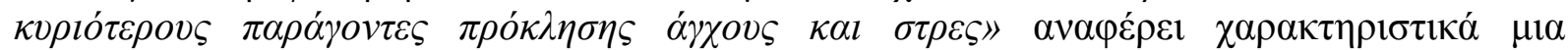

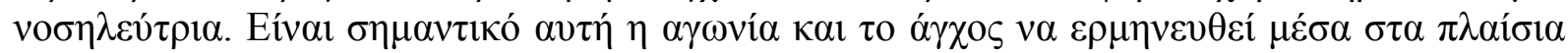

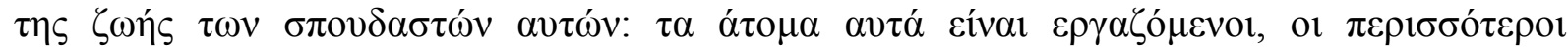

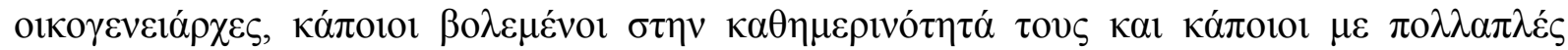

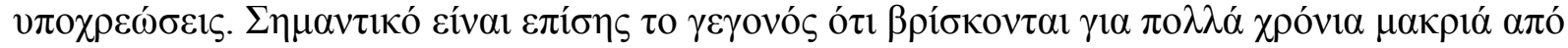

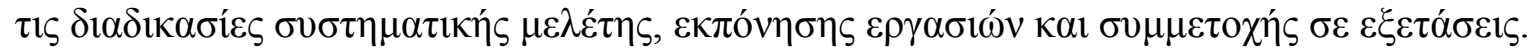

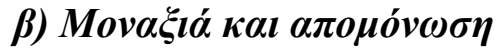

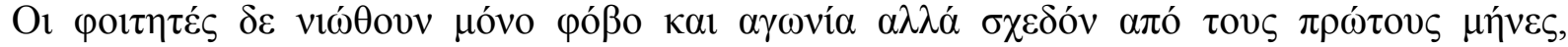

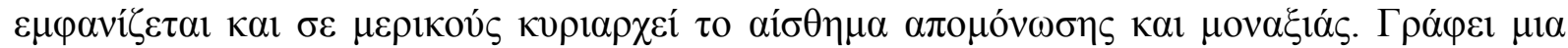

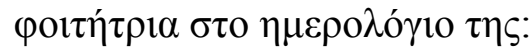

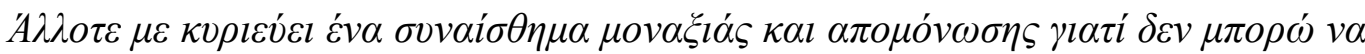

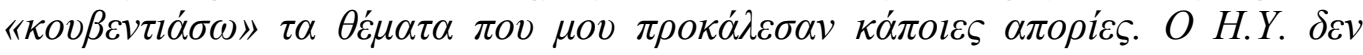

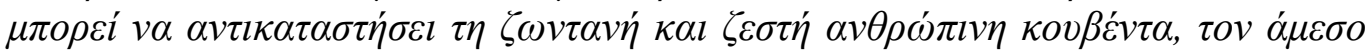

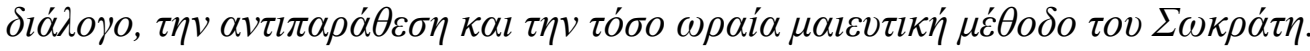

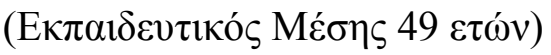

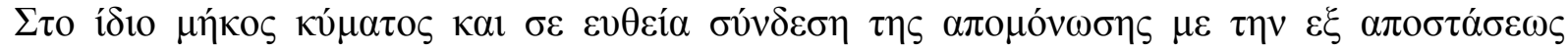

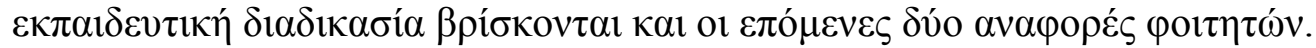

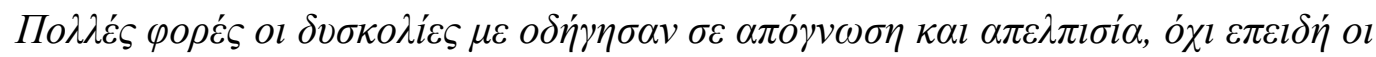

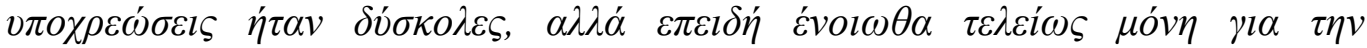

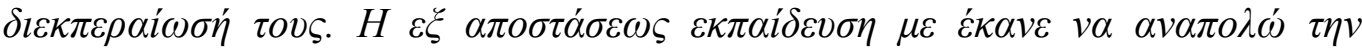

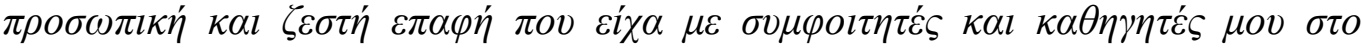
$\sigma \nu \mu \beta \alpha \tau \iota \kappa o ́ ~ \pi \alpha v \varepsilon \pi \iota \sigma \tau \dot{\eta} \mu \imath o$.

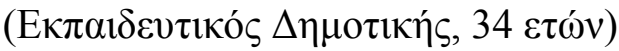

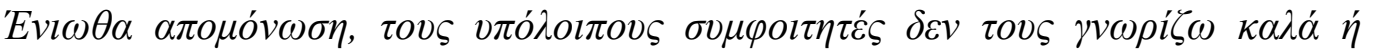

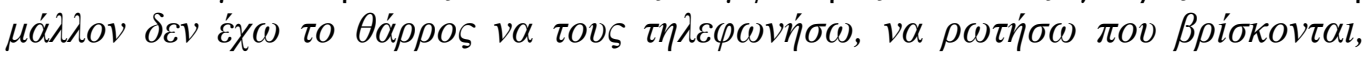

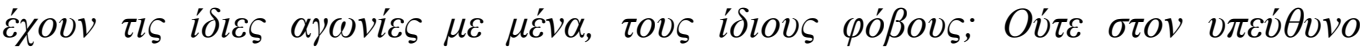

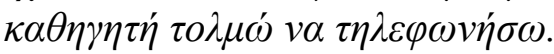

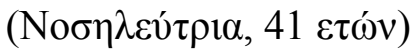

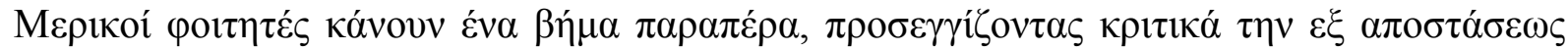

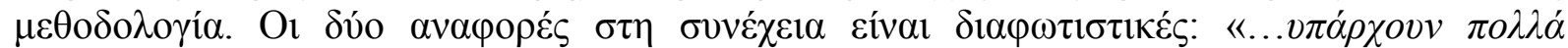

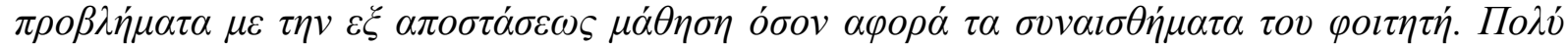

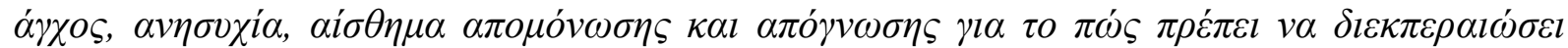

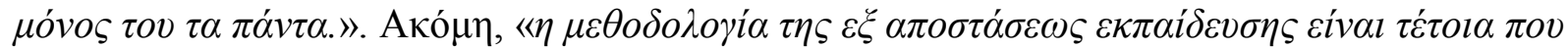

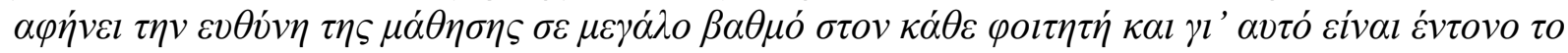

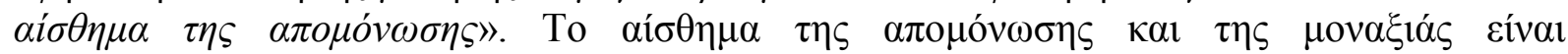

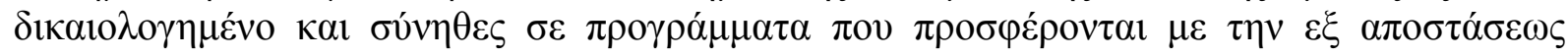




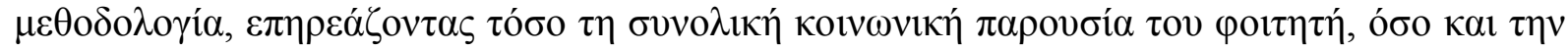

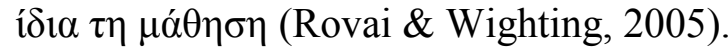

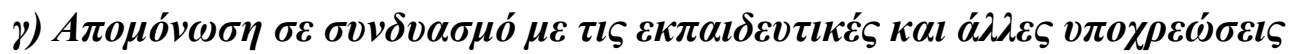

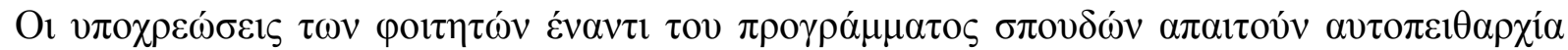

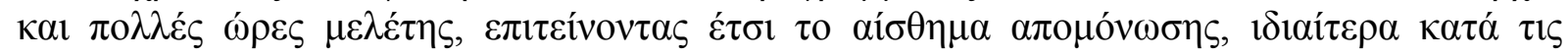

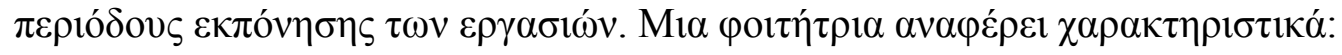

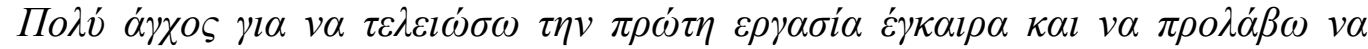

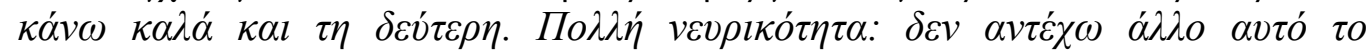

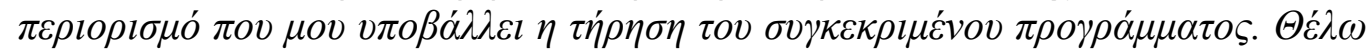

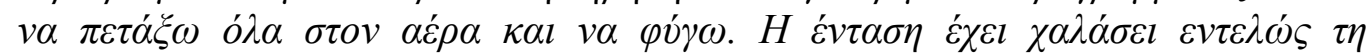

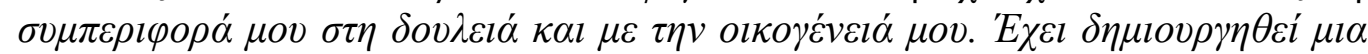

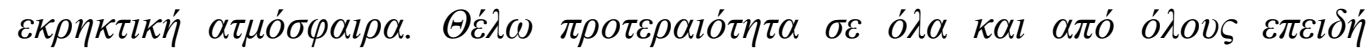

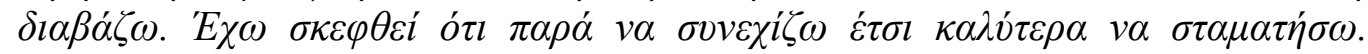

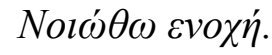

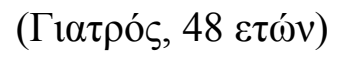

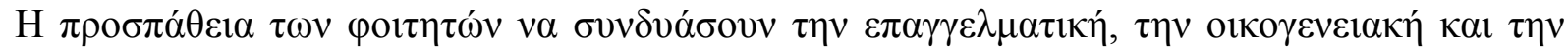

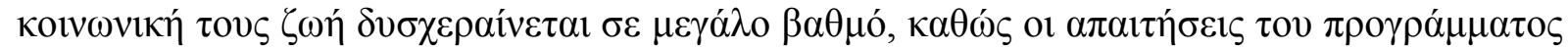

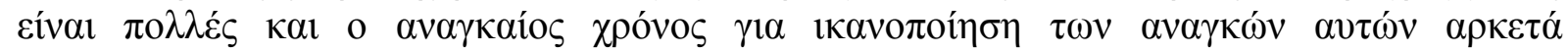

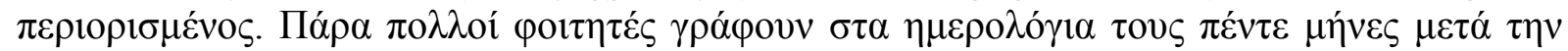

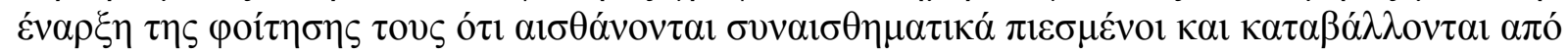

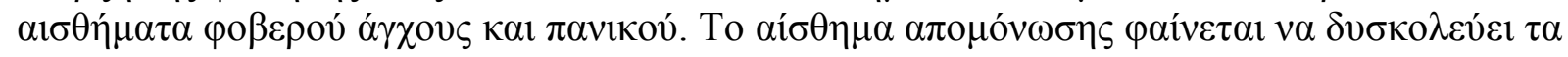

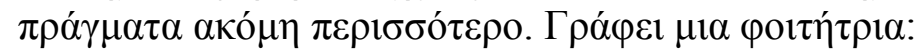

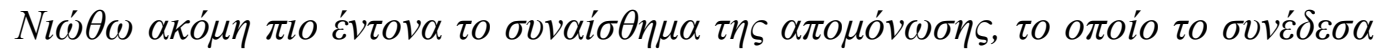

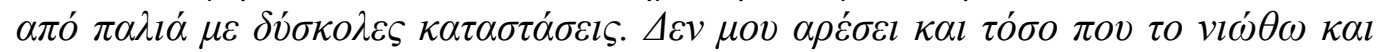

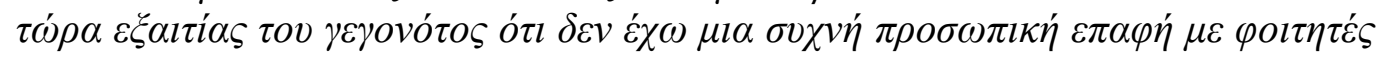

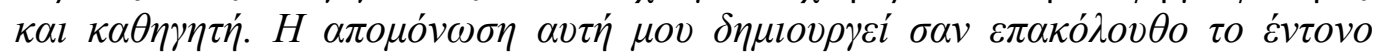

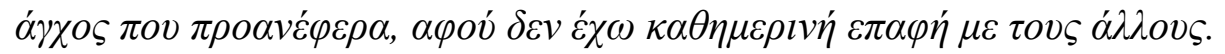

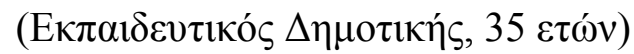

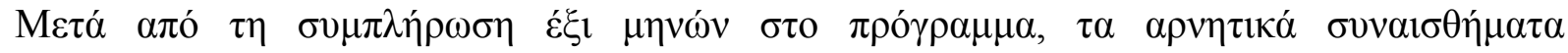

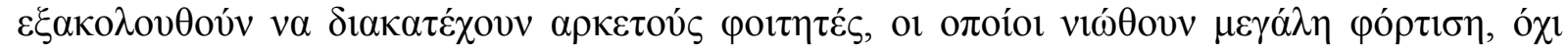

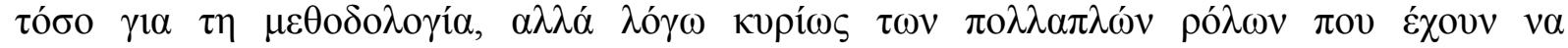

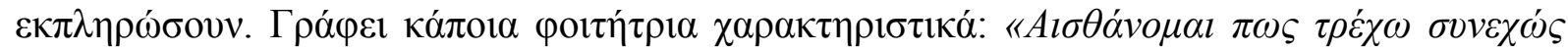

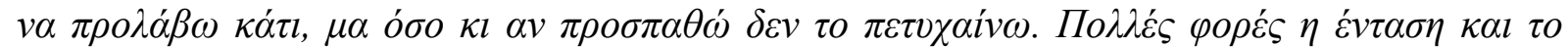

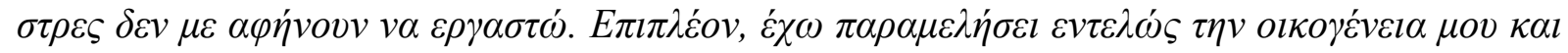

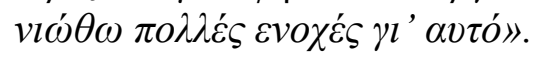

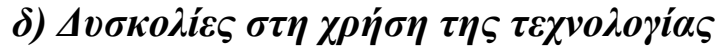

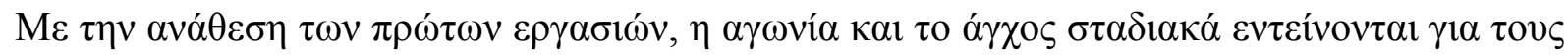

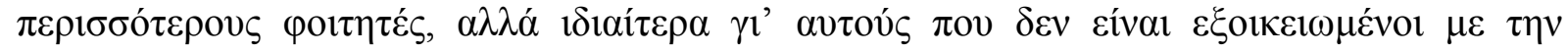

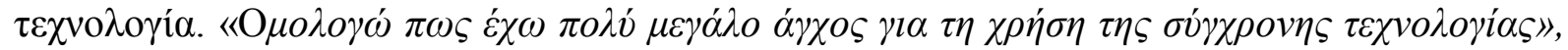

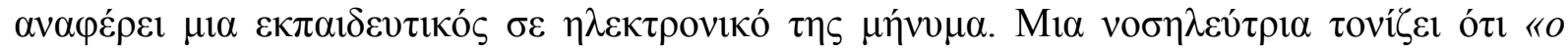

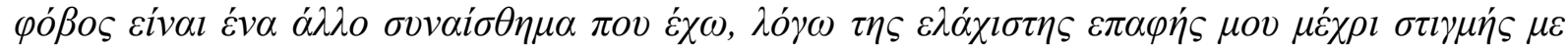

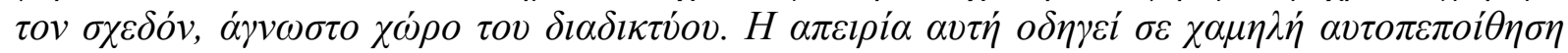




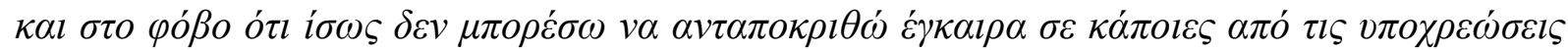

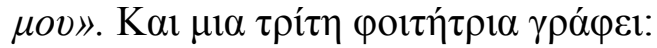

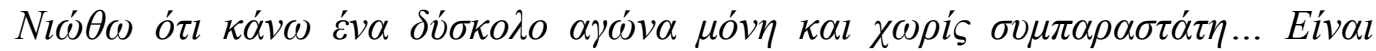

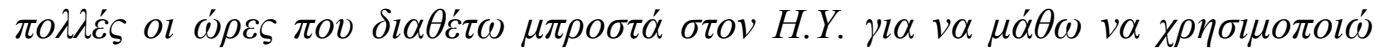

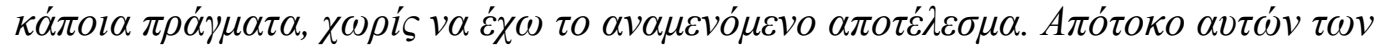

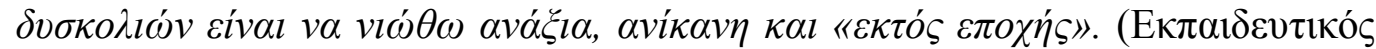

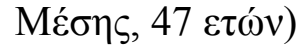

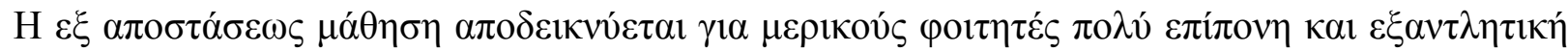

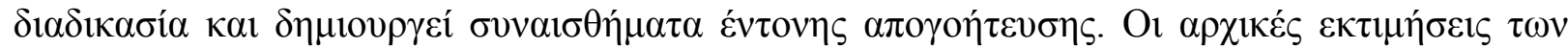

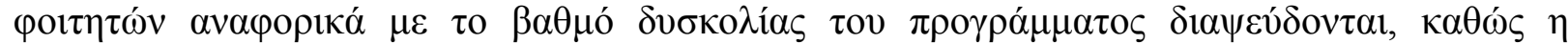

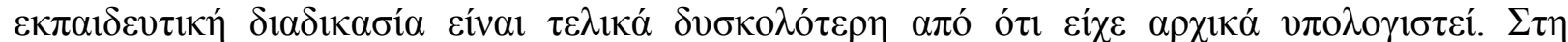

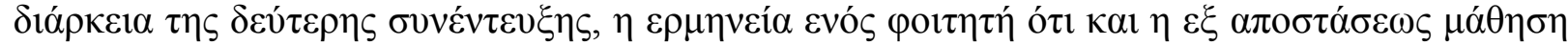

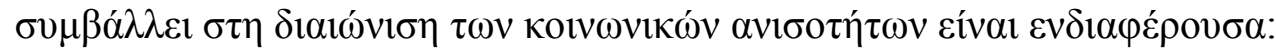

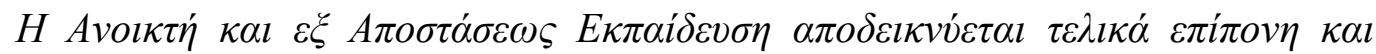

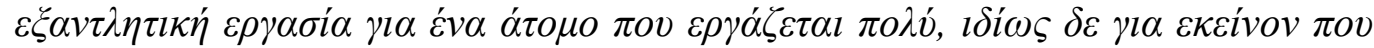

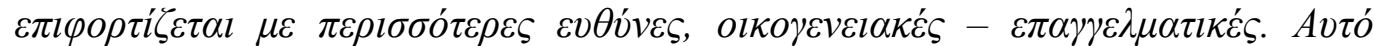

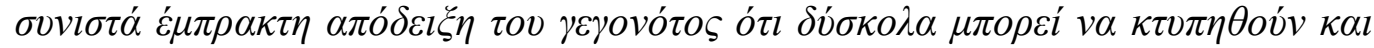

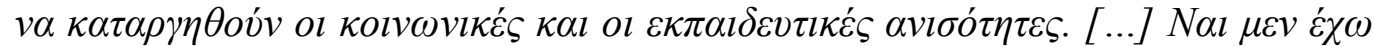

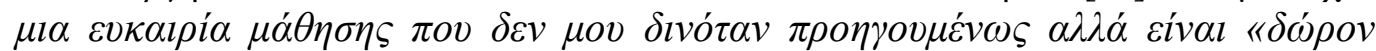

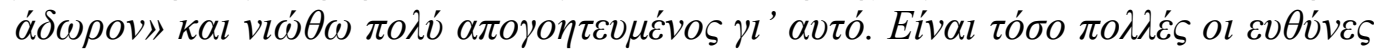

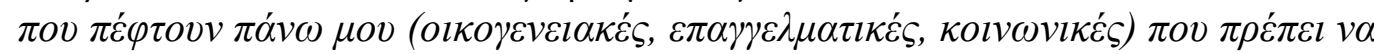

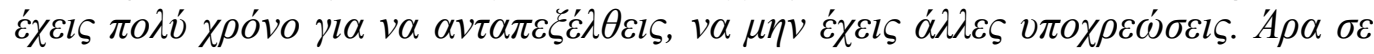

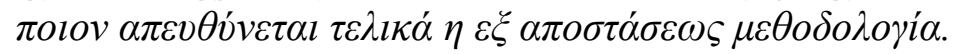

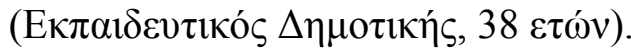

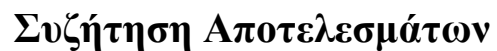

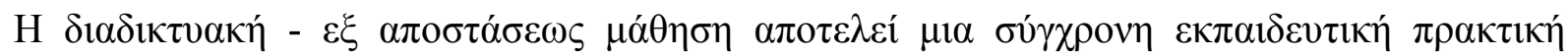

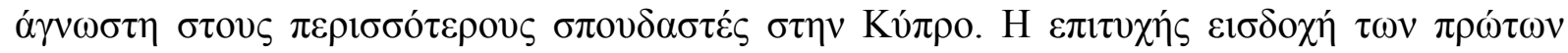

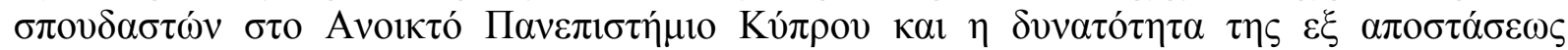

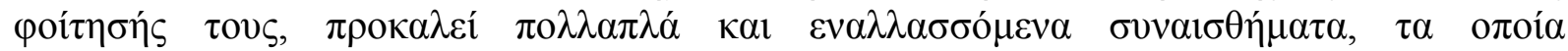

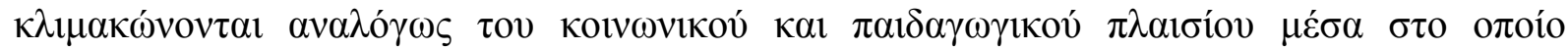

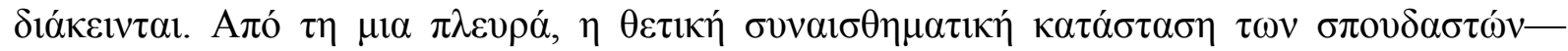

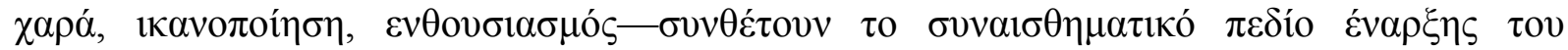

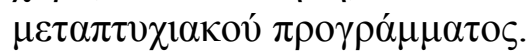

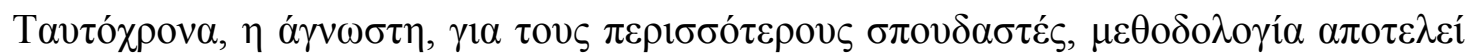

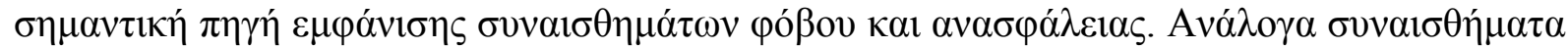

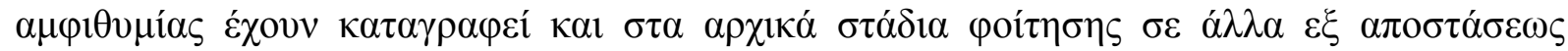

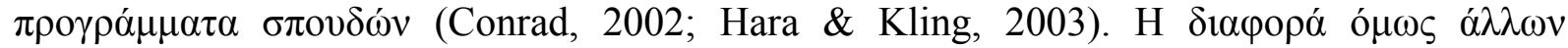

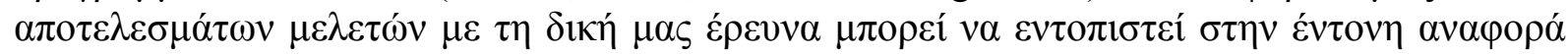

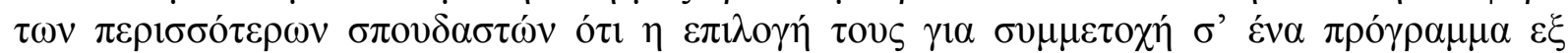

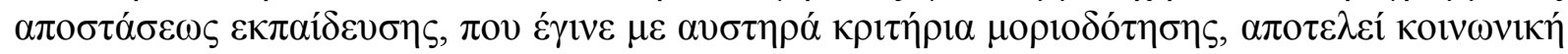

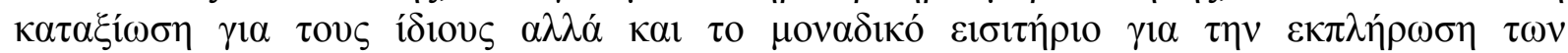

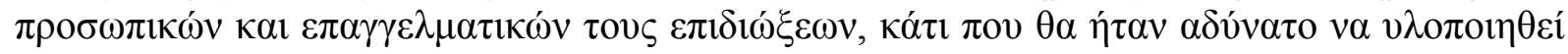
$\chi \omega \rho i ́ \varsigma \tau \eta \nu \varepsilon \xi \alpha \pi \circ \sigma \tau \alpha ́ \sigma \varepsilon \omega \varsigma \varepsilon \kappa \pi \alpha i ́ \delta \varepsilon v \sigma \eta$. 


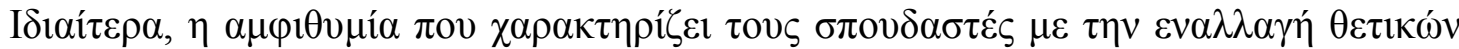

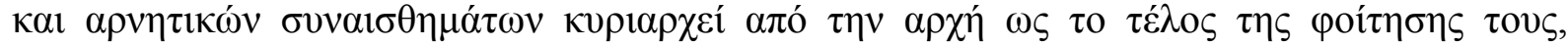

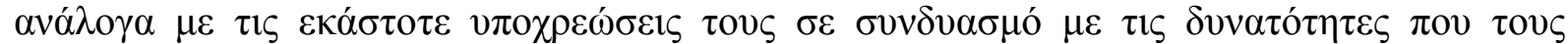

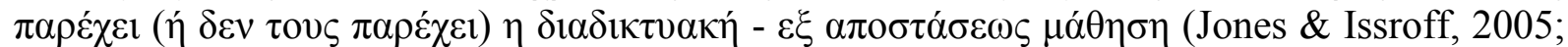

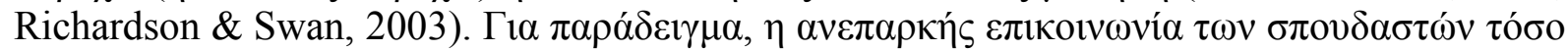

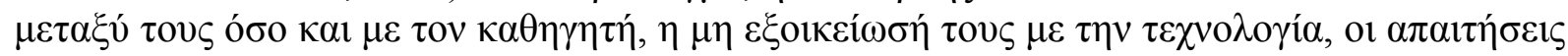

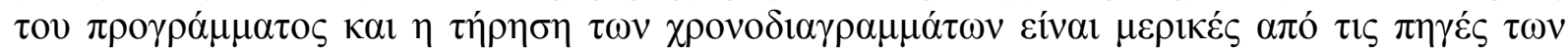

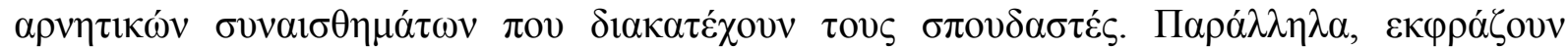

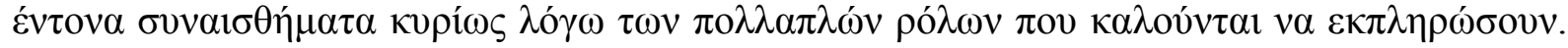

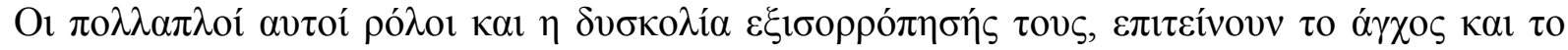

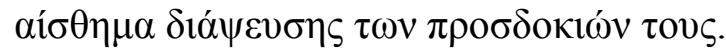

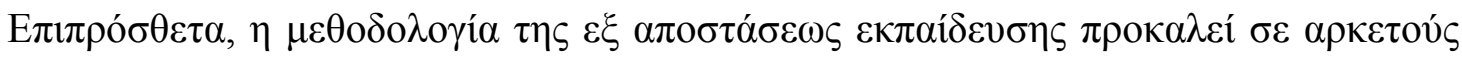

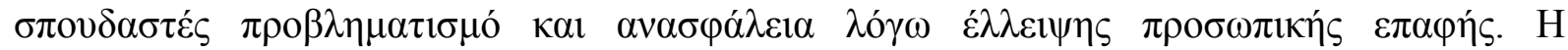

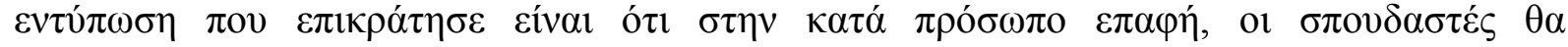

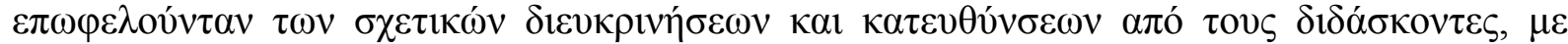

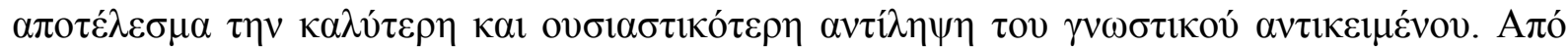

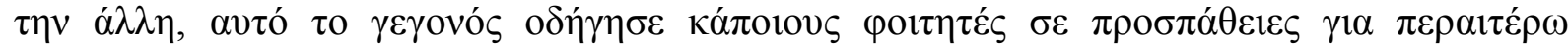

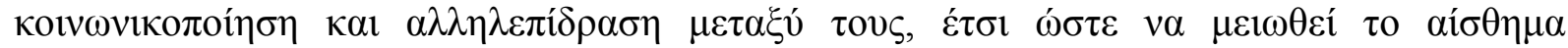

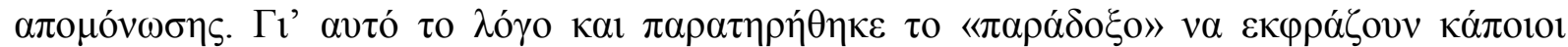

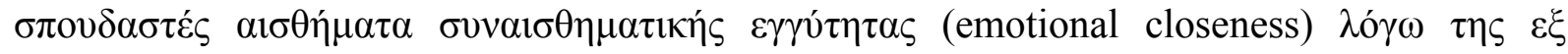

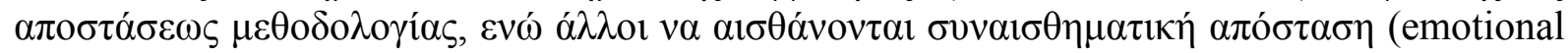
distance) (Ben-Ze'ev, 2003).

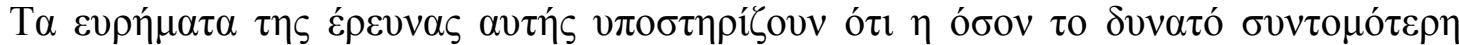

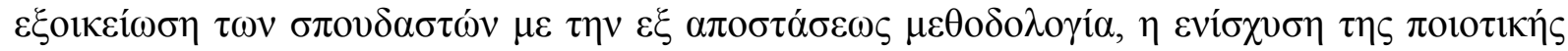

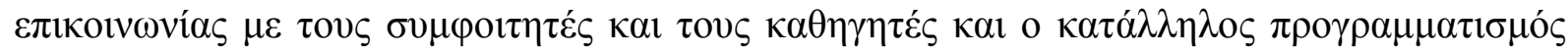

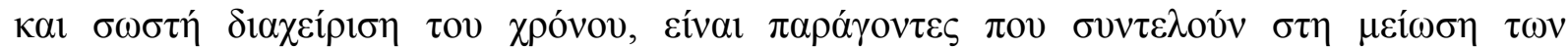

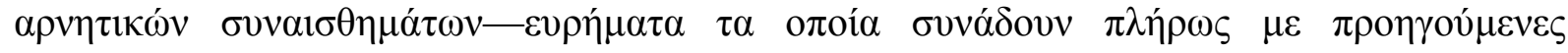

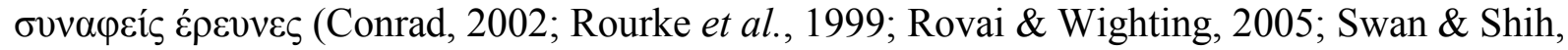
2005).

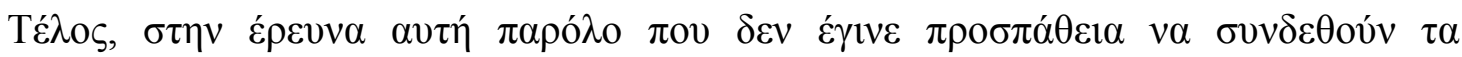

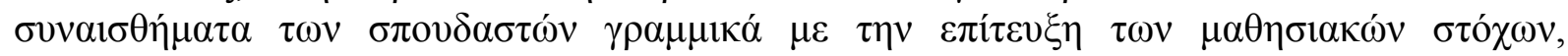

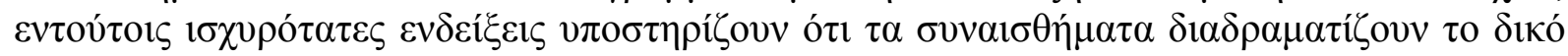

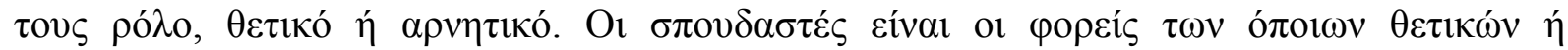

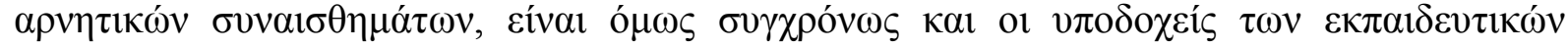

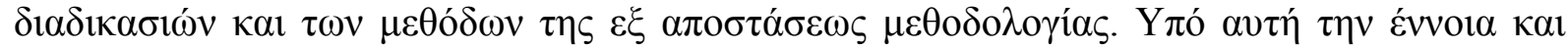

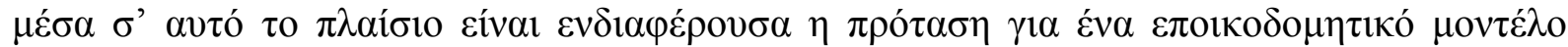

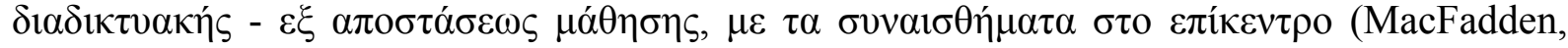

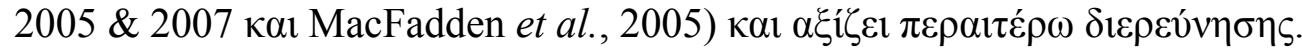




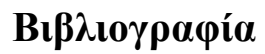

Allan, J., \& Lawless, N., (2003). Stress caused by online collaboration in e-learning: A developing model. Education + Training, 45(8/9), 564-572.

Astleitner, H., (2000). Designing emotionally sound instruction: The FEASP approach. Instructional Science, 28, 169-198.

Ben-Ze'ev, A., (2003). Privacy, emotional closeness, and openness in cyberspace. Computers in Human Behavior, 19, 451-467.

Conrad, D., (2002). Engagement, excitement, anxiety and fear: Learners' experiences of starting an online course. The American Journal of Distance Education, 16(4), 205-226.

Conrad, D., (2005). Building and maintaining community of cohort-based online learning. Journal of Distance Education, 20(1), 1-20.

Dirkx, J. M., (2001). The power of feelings: emotion, imagination, and the construction of meaning in adult learning. New Directions for Adult and Continuing Education, 89, 63-72.

Edwards, R., (1993). Mature Women Students: Separating or Connecting Family and Education. London: Taylor and Francis.

Elliot, J., (1991). Action Research for Educational Change. Philadelphia: Open University Press.

Erickson, F., (1986). Qualitative methods in research on teaching. In M. C. Wittrock (Ed.), Handbook of Research on Teaching (pp. 119-161). New York: Macmillan Publishing Company.

Foucault, M., (1983a). The subject and power: Afterword. In H. Dreyfus \& P. Rabinow (Eds.), Michel Foucault: Beyond Structuralism and Hermeneutics (pp. 208-227). Chicago: University of Chicago Press.

Foucault, M., (1983b). On the genealogy of ethics. In H. Dreyfus \& P. Rabinow (Eds.), Michel Foucault: Beyond Structuralism and Hermeneutics (pp. 229-252). Chicago: University of Chicago Press.

Foucault, M., (1984). The Foucault Reader, P. Rainbow (Ed.). New York: Pantheon.

Glaser, B., \& Strauss, A., (1967). The Discovery of Grounded Theory. Chicago: Aldine.

Hailo, M. P., (2004). Teaching in our pajamas: Negotiating with adult learners in online distance writing courses. College Teaching, 52 (2), 58-62.

Hara, N., \& Kling, R., (2003). Students' distress with a web-based distance education course: An ethnographic study of participants' experiences. Turkish Online Journal of Distance Education, 4 (2),

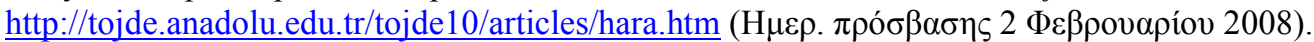

Hubbard, R. \& Power, B., (1993). The Art of Classroom Inquiry: A Handbook for Teacher-Researchers. Portsmouth, NH: Heinemann.

Järvenoja, H., \& Järvelä, S., (2005). How students describe the sources of their emotional and motivational experiences during the learning process: A qualitative approach. Learning and Instruction, 15, 465-480.

Jones, A., \& Issroff, K., (2005). Learning technologies: Affective and social issues in computer-supported collaborative learning. Computers \& Education, 44(4), 395-408.

MacFadden, R., (2005). Souls on ice: Incorporating emotion in web-based education. Journal of Technology in Human Services, 23 (1/2), 79-98.

MacFadden, R., (2007). The forgotten dimension in learning: Incorporating emotion into web-based education. Journal of Technology in Human Services, 25 (1/2), 85-101.

MacFadden, R., Herie, M. A., Maiter, S., \& Dumbrill, G. C., (2005). Achieving high touch in high tech: A constructivist, emotionally oriented model of web-based instruction. Journal of Teaching in Social Work, $25(1 / 2), 21-44$

Ng, K-C., (2001). Using e-mail to foster collaboration in distance education. Open Learning, 16(2), 191-200.

Noffke, S. E., \& Somekh, B., (2005). Action research. In B. Somekh \& C. Lewin (Eds.), Research Methods in the Social Sciences (pp. 89-96). London: Sage.

Noffke, S., \& Stevenson, R. B., (1995). Educational Action Research: Becoming Practically Critical. New York and London: Teachers College Press.

O’ Regan, K., (2003). Emotion and e-learning. Journal of Asynchronous Learning Networks, 7(3), 78-92.

Richardson, J. C., \& Swan, K., (2003). Examining social presence in online courses in relation to students' perceived learning and satisfaction. Journal of Asynchronous Learning Networks, 7(1), 68-88.

Rourke, L., Anderson, T., Garrison, D. R., \& Archer, W., (1999). Assessing social presence in asynchronous text-based computer conferencing. Journal of Distance Education, 14(2), 51-70.

Rovai, A., \& Wighting, M., (2005). Feelings of alienation and community among higher education students in a virtual classroom. Internet and Higher Education, 8, 97-110.

Stake, R. E., (1995). The Art of Case Study Research. Thousand Oaks, CA: Sage.

Strauss, A., \& Corbin, J., (1998). Basics of Qualitative Research (2 ${ }^{\text {nd }}$ ed.). Thousand Oaks, CA: Sage. 
Swan, K., \& Shih, L. F., (2005). On the nature and development of social presence in online course discussions. Journal of Asynchronous Learning Networks, 9(3).

Volet, S., \& Järvelä, S., (Eds.). (2001). Motivation in Learning Contexts: Theoretical and Methodological Implications. Amsterdam: Pergamon.

Wegerif, R., (1998). The social dimension of asynchronous learning networks. Journal of Asynchronous Learning Networks, 2(1), 34-49.

Wosnitza, M., \& Volet, S., (2005). Origin, direction and impact of emotions in social online learning. Learning and Instruction, 15, 449-464.

Zembylas, M., (2002). Constructing genealogies of teachers' emotions in science teaching. Journal of Research in Science Teaching, 39, 79-103.

Zembylas, M., (2004). Young children's emotional practices while engaged in long-term science investigations. Journal of Research in Science Teaching, 41, 693 - 719.

Zembylas, M., (2005). Teaching With Emotion: A Postmodern Enactment. Greenwich, CT: Information Age Publishing. 


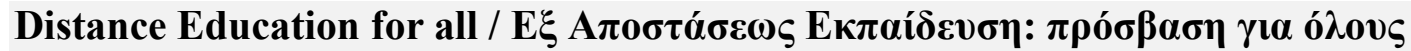

\author{
Anna Koulikourdi

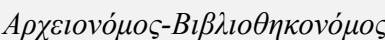 \\ Athens Information Technology (AIT) Institute \\ e-mail:akou@ait.edu.gr
}

\begin{abstract}
Distance education options create learning opportunities for many people but simultaneously, they erect access barriers for some individuals with disabilities. This paper addresses this target group - students with disabilities- and encourages their greater inclusion in the planning and delivery of library services. Furthermore, it identifies the lack of literature on information seeking behavior of people with disabilities and the fact that distance learning literature does not discuss issues of equal access for this vulnerable group of users. For eliminating information disability, this paper pinpoints the essential role of libraries, legislation and assistive technologies and highlights the need for further research and investigation in the fields of on-line accessibility, information seeking behavior of disabled students and disabilities in higher education.
\end{abstract}

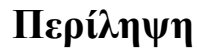

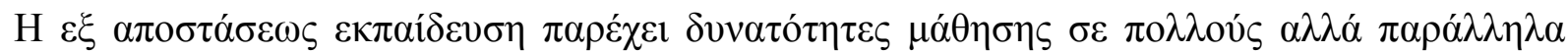

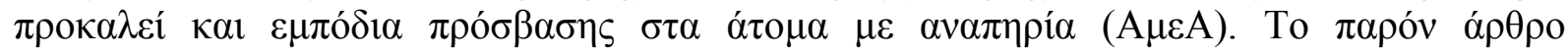

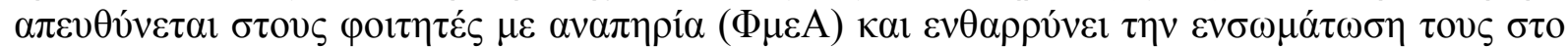

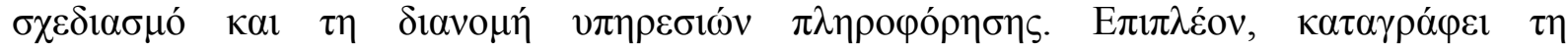

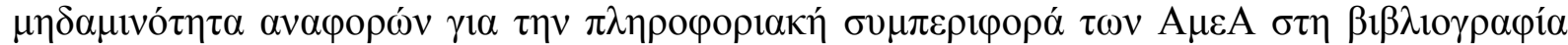

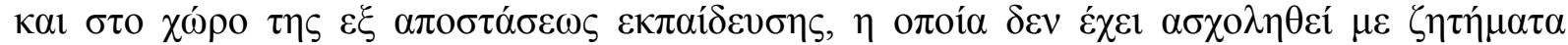

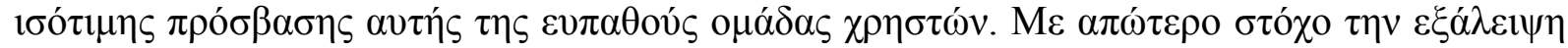

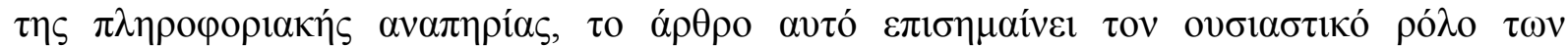

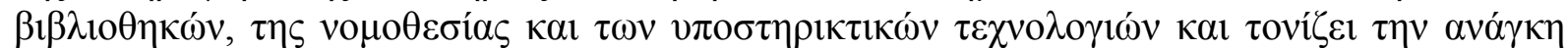

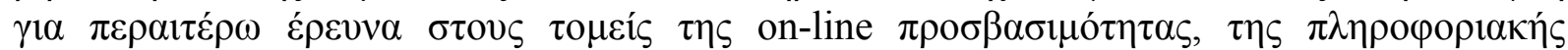

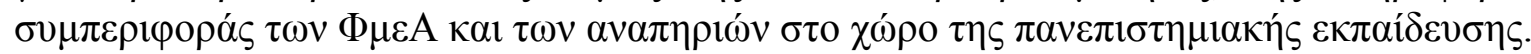

Keywords: Distance Learning, People with Disabilities, Libraries, Accessibility

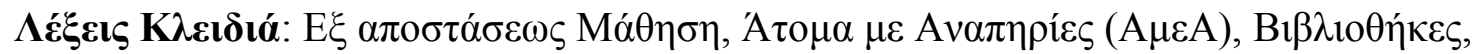

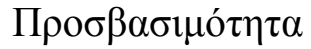

\section{Introduction}

During the last years, increasing numbers of young people with disabilities are attending postsecondary academic institutions (Henderson, 2001; National Council on Disability, 2000). However, these individuals experience far less academic success than their non-disabled peers (Horn and Berktold, 1999). The impact of technology on the delivery of education is clearly demonstrated in the tremendous growth of on-line distance education programs and webbased distance learning course offerings. It is assumed by most instructors and administrators that anyone with computer and Internet connection can access these courses and the supplementary materials offered by libraries, university departments, businesses and other 
groups. In the global information society, growing numbers of individuals with disabilities have access to computers, assistive technology and the Internet. However, some of them still do not have access to all the content delivered via the Internet because of the inaccessible design of electronic resources.

\section{People with Disabilities (or Differabilities)}

According to the Greek Act 1566/85, people with disabilities are defined as those that due to physical, mental or social reasons perform retardations, disabilities or disorders and they have serious difficulty in getting involved in education, being productive and integrating with society. The Greek Parliament has declared $3^{\text {rd }}$ December as National Day of People with Disabilities (Act 2430/96) with the intention of eliminating the disability gap.

There are several categories of people with disabilities such as the blind or people with visual impairments (VIP), learning disabilities, pre-lingual and post-lingual deaf people, deafblind, individuals with physical impairments, developmental disabilities, syndrome Down, attention deficit disorder, multiple sclerosis, autistic people, etc. Disabilities are divided to hidden, visible and temporary ones. The category of hidden disabilities is the most difficult because they are not easily perceived by instructors, librarians, etc.

There is also a broader category of people with disabilities, the "print-disabled" or the "printhandicapped", which includes all those that, for the above mentioned reasons, are unable to handle and use the standard print. Students with disabilities need access to the same resources as their able-bodied peers (Kavanagh, 1994). In USA disabled college students need access not only to textbooks but also to newspapers, professional journals, government documents and other reference sources (McNulty and Suvino, 1993).

Print-disabled people are not able to use libraries designed for the average, able-bodied, traditional student. These people need alternative format materials (talking books, recordings for the blind, braille material, e-braille, adapted literature, digital talking books, captioned material and videos, easy-to-read literature, etc) and accessible web-based resources. But, how many distance learning programs offer such accessible material to these students?

For example, people with visual impairments on average prefer studying humanities, social sciences, psychology and not areas of science, such as engineering, chemistry, physics, biology, and mathematics. Those areas have traditionally been less accessible to students with visual impairments in particular, because the complex visual information generally associated with these disciplines has not been readily accessible (Coonin, 2001).

People with disabilities have a lot of attributes of information poverty and social exclusion, such as poor education, low levels of literacy and income. Apart from the social exclusion, they also experience digital inclusion, disability gap and live in a world of information apartheid. But soon all education stakeholders (colleges, institutions, libraries, instructors, providers of content, etc) have to recognize that the functional disability of people with disabilities must stop implying also their information disability. 


\section{Distance Learning and Students with Disabilities}

It is widely known that computers and Internet are less available to people with disabilities, that's why we hear often about information rich and poor people and computer "have" and "have-nots". Without access to computers and Internet, students with disabilities cannot participate in web-based learning options. They have to deal with innumerous problems, to struggle for gaining access to basic resources and material for their e-courses and because of those, a lot of them resign. It is likely that web resources and other information technology will erect barriers to some people unless universal design considerations are made as resources are being developed. Applications of universal design to instruction in general have begun to appear in the literature (Bar and Galluzzo, 1999; Bowe, 2000; Burgstahler, 2000; Center for Applied Special Technology, 1999). Most articles and books about distance learning design, however, do not discuss universal design principles or specific access issues for students and instructors with disabilities. The majority of articles that discuss the application of universal design to Web pages thus far have appeared in library publications (Schmetzke, 2001).

Core distance education journals, such as "The American Journal of Distance Education", "Journal of Instructional Science and Technology", "Virtual University Journal", "Online Chronicle of Distance Education and Communication" and the "Journal of Library Services for Distance Education" do not reflect any concern about the accessibility of online resources for people with disabilities or the "future of library services for off-campus/distance education" (Schmetzke, 2001). Pertinent special-theme issues in other disciplines are not any different in this regard, such as the special issue on "Telecommunication, Distance Learning, and the World Wide Web" in Technical Communication Quarterly.

As for library literature, we searched 400 library-related publications for identifying material relevant to accessible distance learning or distance education so as to examine the dearth or richness of resources on this subject. The results were only...two relevant publications (Burgstahler, 2002; Schmetzke, 2001).

The largest division of ALA (American Library Association), the Association of College and Research Libraries (ACRL) revised in 2000 its 1990 "Guidelines of Distance Learning Library Services", where it gives the definition of "distance library services". Although the "distance learning community" addressed there covers:

“...all those individuals and agencies, or institutions, directly involved with academic programs or extension services offered away from a traditional academic campus or in the absence of a traditional academic campus, including students, faculty, researchers, administrators, sponsors, and staff....(ACRL, 2000)"

The current guidelines do not include standards for making electronic resources accessible to individuals with disabilities (ACRL, 2000). The ACRL has clearly missed an opportunity to model for its members how a commitment to accessibility can be integrated into distance learning policy. In the literature, there are accessibility policies of academic, public, special and college libraries but it is quite scarce to find policies for designing accessible distance learning courses and web-based resources. Few colleges and universities have policies and guidelines that specifically address the accessibility of distance learning classes. Because of this, it is not surprising that many college, university, library and distance learning webpages 
are not fully accessible to individuals with disabilities (Craven, 2000; Evans, 2000; Guthrie, 2000; Lilly and van Fleet, 2000; Rowland, 2000; Schmetzke, 2001).

Distance learning options create learning opportunities for many but erect access barriers for some individuals with disabilities. Making a distance learning course accessible to everyone minimizes the number of legal challenges and complaints, supports a diverse student body, creates a better learning environment for everyone and is the right thing to do.

Even though the number of students with disabilities gets increased continually, they continue to constitute a minority to the general student body. For this reason, the available information for their needs and experiences are quite limited. There are no studies that record and monitor their real-life learning and educational conditions so as to suggest further improvements and help them participate more actively in their colleges and universities.

This ignorance is due to the fact that students with disabilities rarely enter academic institutions. Deaf students constitute a small but increasing number in college communities. For example, academic libraries have not taken the needs of deaf students into consideration because the minority of them reaches this educational level. Most of them get enrolled to colleges with little or no knowledge of their institution's library systems and resources. This situation gets even more complex, since most librarians ignore them accordingly, thus there is no interaction which could lead to cooperation and equal information services.

The result is that students with disabilities remain unserved, disadvantaged and underprivileged. There is online distance education anytime, anywhere but not for everyone while disabled students should be a part of their institutions' libraries and not apart.

In library literature, the researcher may find paradigms and personal narratives of students with disabilities. Damon Rose (from London) finds ridiculous the fact that blind students have to scan a whole book first (a process that may take up to 8 hours which means a whole working day) before reading it because it is not available in accessible format while their fellow-students get the information that they want just by entering the library or using several digital libraries. Blind students must work 10 times even harder than their sighted fellowstudents, that' s why most of them get lower grades because of their superhuman effort.

\section{The Library's Role}

The history of library services to people with disabilities is recent and comparatively short. The most important challenge that libraries and information services deal with in our information age is "inclusiveness". For facilitating students with disabilities succeeding in the distance learning programs of their choice, libraries have to:

- research the current situation

- focus on students with disabilities and respond to their specific needs

- meet access challenges and find solutions for enabling and integrating disabled students

- take steps towards accessible resources

- develop policy statements

- adopt universal design principles

- involve all education stakeholders

- evaluate their own progress

- deliver state-of-the-art services

- assign responsibilities and establish basic processes 
- adopt guidelines for accessible electronic and information technology

- disseminate information on accessibility

- procure accessible products so as to remove barriers to access

- develop successful strategies for bridging information gaps putting person first, disability second

- find the best fit between user, environment and technology

- act for the removal of some copyright barriers

These methods will provide a new vista on library services, will open new worlds of information, expand the existing library systems and finally, by implementing all these, libraries will hopefully be transformed from reading places to access points.

The literature of library and information science (LIS) discusses consistently the need for libraries to interact with their communities. But this discussion tends to focus on general populations (faculty, students, staff) and not on the so-called special ones (people with disabilities). For this reason, librarians should change their attitude, way of thinking and focus of interest. Besides, attitudinal barrier is the most often cited one in providing equal services to people with disabilities. Librarians should focus not only on well-functioning and welleducated students but also on weak readers. Even more, they should be proactive and they should not assume disabled students' needs, aspirations and expectations.

It is worth noting that all the above do not imply that all the responsibility is solely left within the library. On the contrary, the institutes that offer the distance learning programs and their stakeholders have to understand that students with disabilities pose a challenge and not a problem. They have to respect and listen to their needs, to include them in every phase of their educational process and plan for them, not without them.

Last but not least, students and instructors with disabilities should inform libraries and other content-providers when their resources are not accessible and should demand access to the content. They have to pinpoint the problem and request equitable educational conditions, since their motto is "nothing about us without us".

\section{Legislation}

One of the most catalytic factor that affects library services for people with disabilities is legislation of each country. Most initiatives for equal information services for people with disabilities, access for all and the increasing number of students with disabilities to colleges and universities seem to result from the relevant disability legislation, such as Americans with Disabilities Act (ADA, 1990), Australian Disability Discrimination Act (1992), Disability Discrimination Act (1995) of United Kingdom and Special Educational Needs and Disability (2001). The law and student demands spur colleges to seek new design and approaches.

Since the beginning and mid of 1990, there have been several movements encouraging the social inclusion of people with disabilities, in which post-secondary institutions and their libraries have responded positively.

The sensitization of Greek nation for the disabled started almost at the end of the first half of $20^{\text {th }}$ century (Darais, 2001) while until then provision for them was non-existent. In comparison with other countries, the right to education and information was recognized only a few decades ago. In particular, according to Act 904 passed in 1951 (the first law aiming at the support of the disabled), Greek government for the first time is obliged to take care and 
ensure the education of people with visual impairments. Because of this delay, Greek literature referring to the information seeking behavior of people with disabilities is quite meager.

Currently, even though United Stations' declarations referred to unhindered access to services, activities, books, leaflets and generally to information, students with disabilities continue to experience learning, academic and thereby, social exclusion (Kalantzi-Azizi \& Tsinarelis, 1994). Unfortunately, legal barriers to full access have not been eliminated, especially when the copyright issue intervenes.

\section{On-line Accessibility and Information Seeking Behavior of People with Disabilities}

Until recently, information on accessibility of web-based library resources was scarce. It was not until 1996 that accessible web design emerged as an issue in the professional library literature and in 1998 researchers began collecting data on the accessibility of library web sites and on-line resources (other than webpages) such as e-journals, databases, etc. Until 2002, there were few things published in the field of online accessibility research. Since then, several studies have been conducted so as to record accessibility and design issues and evaluate the accessibility of campuses, academic and public library webpages. Library literature presents a wealth of surveys of library services, web accessibility survey sites, manuals of best practice, case studies of individual libraries, etc.

As for the information seeking behavior or on-line strategies of the disabled, there is lack of literature dealing with these issues, although web accessibility is largely influenced by those parameters. For this reason, there is need to approach users with disabilities and explore their information seeking behavior so as to offer them information equity. Students with disabilities constitute a distinct and important group that, from time to time, have unique service expectations. By exploring their information behavior, we are going to escalate the information gap between the print-disabled and the non-handicapped and optimize library's performance and delivery of information services. There is room for additional research that might investigate their varying information and communication needs and especially their interaction with on-line technologies, Internet and web-based resources (since the very visual nature of most web resources makes them potentially inaccessible).

In library professional literature, there is considerable discussion of Internet benefits and possibilities for people with disabilities. "Internet has the power to change the life of the disabled" and "microcomputers have been voted as the new saviors for people with disabilities". The positive influence of Internet in the life of people with disabilities is obvious (Grimaldi and Goette, 1999; Taylor, 2000). But, even though there are several studies that have examined the Internet's use, there is none that records systematically the way that people with disabilities use online services and a pool of other dimensions, such as:

- Which Internet services do they use more often?

- Which are the main reasons that they use the Internet?

- How does the level and type of disability affect user's perception of multimedia and web-based resources?

- Which are the on-line retrieval strategies that they adopt?

- Are they enough computer literate and what can be done so as to succeed it?

- Which is the searching process for disabled users of the web?, etc. 
Electronic media should be a boon for people with disabilities but few colleges embrace the technologies that could help them. The loss of a sense influences the way a person reads, gets informed and more generally communicates. In such circumstances, people with disabilities rely on the rest of their senses and use information channels through their sight, sound and touch. Because of their difficulty in searching, browsing, retrieving and selecting material from resources designed for the able-bodied, it is quite obvious and logical that they choose the easiest information paths for them, since they do not have a lot of information choices. As everybody, they want to lead a productive life, to keep their mind alive and get educated. For example, if a blind student wants to access web-based resources, he would prefer doing it on his own, without relying on human readers or other mediators. People with disabilities desire real-time access to information and dream of being independent and self-determined learners.

\section{Assistive Technologies}

Sometimes accessing web-based information depends on the appropriate adaptive or assistive technology that the institute or the user has at his disposal. Of course assistive technology cannot overcome barriers such as poor coding or design. When a web-based resource is poorly designed, it is not going to be accessible even if the user has the most advanced assistive technology. Nowadays, there is a maze of adaptive and education-based information technology, such as:

- Magnifiers

- Enlargement software

- Video magnifiers

- Screen readers

- Speech synthesis

- Braille display (this device translates all the information that appear on a Windows screen in a series of eight Braille dots/ characters)

- OCR

- Braille embosser

- CCTV (Closed Circuit Television)

- Electronic notetakers

- Braille translation software

\section{Conclusion}

Students with disabilities, though present on campus previously in the past, have only recently become a focus of this diversification. These students present vastly different learning styles, physical accessibility needs and sensory differences that distance learning providers must take into consideration if they want to offer accessible and equal web-based courses and material. They need to stop making assumptions about this population and listen to its needs and expectations so as to result to the development and improvement of services. Conclusively, design for all and user centered design are the only design approaches that distance-learning education has to adopt. The target of their efforts should be the user's enforcement by placing the needs and demands of the disabled in the centre of the design process. 


\section{References}

Americans with Disabilities Act of 1990. (1990). The Americans with Disabilities Act of 1990. Retrieved February 12, 2006, from www.usdoj.gov/crt/ada/statute.html

Association of College and Research Libraries. (2000). Guidelines for Distance Learning Library Services. Retrieved December 18, 2006, from www.ala.org/acrl/guides/distlrng.html

Bar, L. \& Galluzzo, J. (1999). The accessible school: universal design for educational settings . Berkeley, CA:MIG Communications

Bowe F.G. (2000). Universal design in education. Westport, CT:Bergin and Garvey

Burgstahler S. (2000). Universal design of instruction. Seattle: University of Washington

Burgstahler, S. (2002). Distance learning: the library's role in ensuring access to everyone. Library Hi Tech, 20 (4), pp. 420-432

Center for Applied Special Technology Universal Design for Learning. (1999). CAST Universal Design for Learning. Retrieved November 12, 2006, from www.cast.org/udl/UniversalDesignforLearning361.cfm

Coonin, B. (2001). Enabling scientists: serving sci-tech library users with disabilities. Issues in Science and Technology Librarianship, 32

Craven, J. (2000). Electronic access for all: awareness in creating accessible Websites for the university library. University of Dundee: DISinHE

Darais, K. (2001). People with disabilities in society. Virtual school, 2 (2-3). Retrieved February 20, 2007, from http://www.auth.gr/virtualschool/2.2-3/Praxis/DaraisDisabilityInSociety.html

Evans, C. (2000). Universal access, the ADA, and your library Web page. Arkansas Libraries, 57 (1), pp.19-24.

Grimaldi, C, Goette, T. (1999). The Internet and the independence of individuals with disabilities. Internet Research: Electronic Networking Applications and Policy, 9 (4), pp.272-80

Guthrie, S.A. (2000). Making the World Wide Web accessible to all students. Journal of Mass Communication Educator, 55 (1), pp.14-23.

Henderson C. (2001). College Freshmen with Disabilities: A Biennial Statistical Profile. Washington, DC: American Council on Education

Horn, L., Berktold, J. (1999). Students with disabilities in postsecondary education: a profile of preparation, participation, and outcomes. Education Statistics Quarterly, 1 (3), pp.59-64.

Kalantzi-Azizi, A., Tsinarelis, G. (1994). Students with Special Needs in University of Athens. In M. Kaila, N. Polemikos \& G. Philippou (Eds.) People with Disabilities (pp752-758). Athens: Ellinika Grammata

Kavanagh, R. (1994). Supporting blind people in education and employment. In 60th IFLA General Conference, 21-27 Aug. Cuba: IFLA

Lilly, E., Van Fleet, C. (2000). Wired but not connected: accessibility of academic library home pages. In W. Arant \& P.A. Mostley (Eds.), Library Outreach, Partnerships and Distance Education: Reference Librarians at the Gateway (pp5-28). New York, NY: The Hallworth Press

McNulty T \& Suvino, D. (1993). Access to Information. American Library Association

National Council on Disability. (2000). Transition and Post-school Outcomes for Youth with Disabilities: Closing the Gaps to Post-secondary Education and Employment. Washington, DC: National Council on Disability

Rowland, C. (2000). Accessibility of the Internet in postsecondary education: meeting the challenge. Retrieved July 20, 2006, from www.webaim.org/articles/whitepaper

Schmetzke, A. (2001). Online distance education- anytime, anywhere but not for everyone. ITD Journal, 7 (2)

Taylor, H. (2000). How the Internet is improving the lives of Americans with disabilities. Retrieved July 26, 2006 from http://www.harrisinteractive.com/harris poll/index.asp?PID=93 


\title{
What kind of Haptic devices and applications are needed in education? Requirements, Specifications and hands-on experience derived from an IST project
}

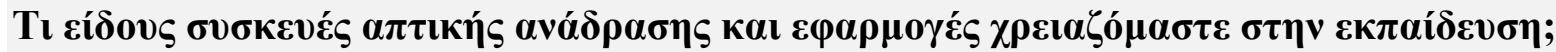

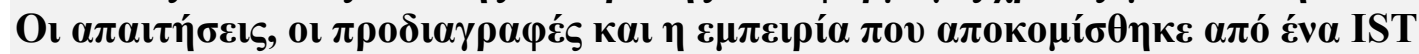
$\pi \rho \hat{\gamma} \boldsymbol{\rho} \alpha \mu \mu \alpha$

\author{
S.P. Christodoulou ${ }^{1}$, D.M. Garyfallidou ${ }^{2}$, \\ G.S. Ioannidis $^{2}$, T.S. Papatheodorou ${ }^{1}$, E.A. Stathi ${ }^{1}$ \\ e-mail:spc@hpclab.ceid.upatras.gr,D.M.Garyfallidou@upatras.gr, \\ gsioanni@upatras.gr,tsp@hpclab.ceid.upatras.gr,stathie@hpclab.ceid.upatras.gr \\ ${ }^{I}$ HPCLab, Computer Engineering \& Informatics Department, \\ ${ }^{2}$ The Science Laboratory, School of Education, \\ University of Patras, 26500 Rion, Patras, Greece
}

\begin{abstract}
In this paper the question whether Haptics technology (virtually touching objects and feeling forces), could be effectively implemented in the teaching procedure is studied, as well as if this technology can be used in improving the understanding of certain scientific concepts. Under the framework of an IST European program called MUVII, a new haptic device was designed especially for educational purposes and a prototype was implemented. This device was designed after thorough consideration of the educational needs. During this process, the most important parameters of the end user requirements were drawn up, and the functionality of the end product was discussed with a team of educational experts (the team also included teachers). These requirements were converted into specifications for a large scale system, called the Interactive Kiosk Demonstrator (IKD). IKD's objective was to demonstrate new interactive paradigms forming a novel integration of the following modalities: 3D-vision, 3Daudio and haptic (force, torque, and tactile) feedback. For the application software design (focusing especially on the context of science education) the educational needs of the end users were thoroughly analyzed and evaluated and, subsequently, some applications were designed and developed. These applications have been used and tested by students of different ages, and their teachers, for a period of over three months in order to divulge the benefits and the drawbacks of the haptic device as well as the applications. Some remarkable results were derived from the users' feedback, which support the view that haptic interfaces can be significantly beneficial to the students' education.
\end{abstract}

Keywords: Haptic Devices, Science Educational Applications, Specifications, User requirements, Virtual Reality.

\section{Пєрí $\eta \psi \eta$}

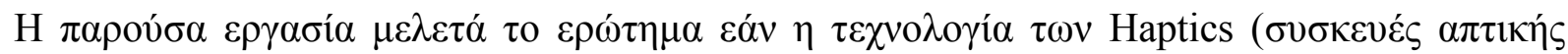

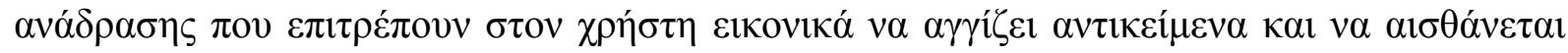

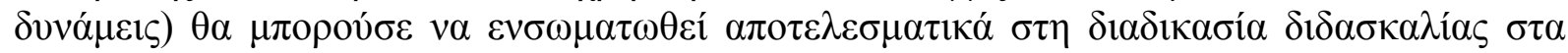

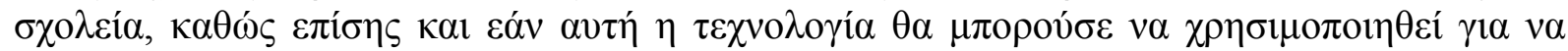

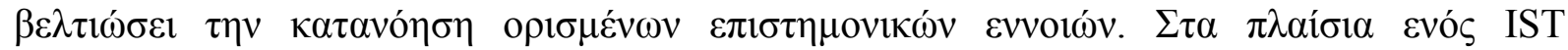

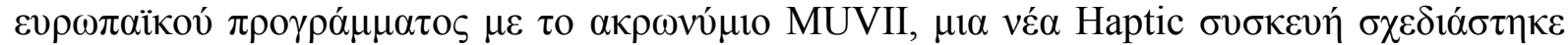

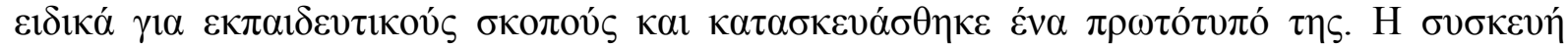

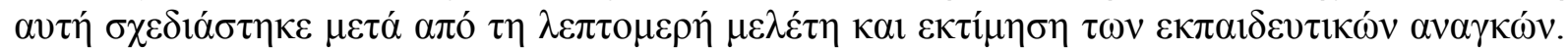

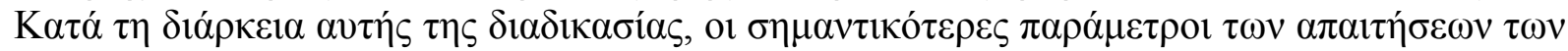




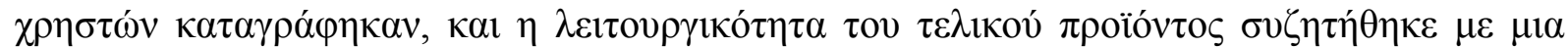

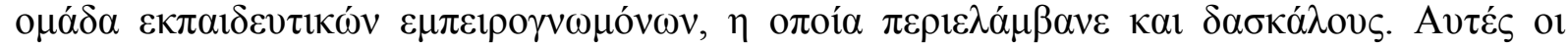

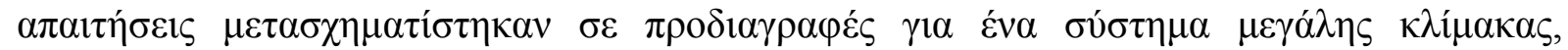

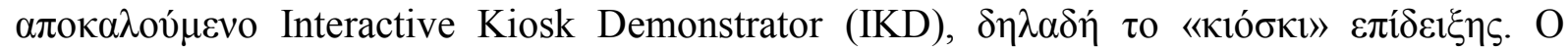

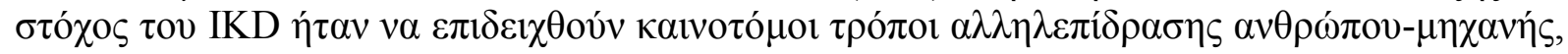

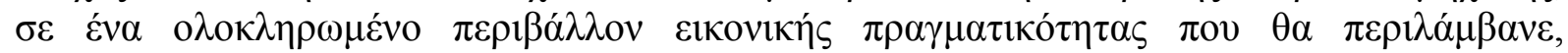

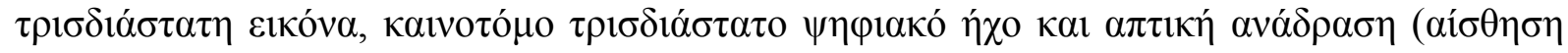

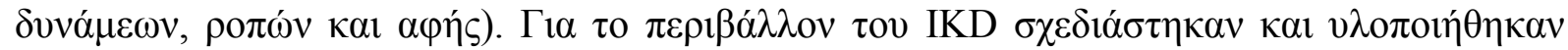

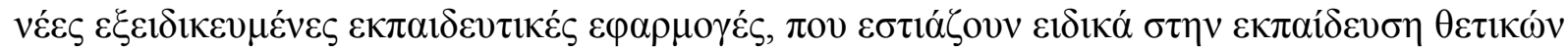

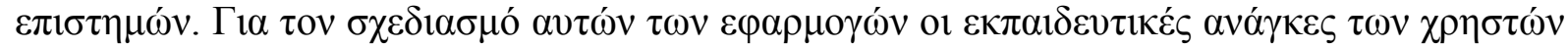

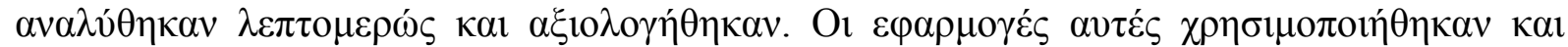

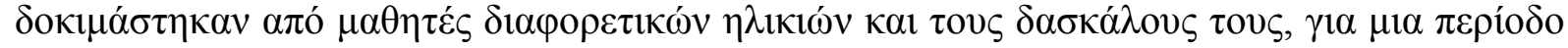

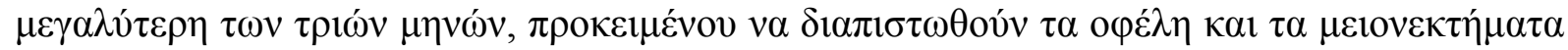

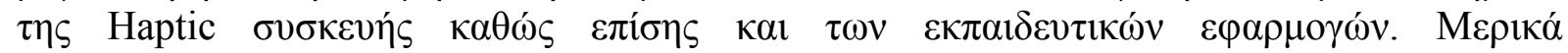

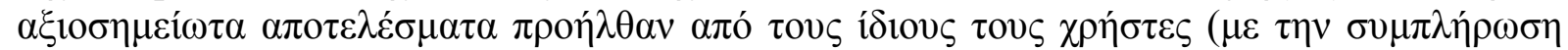

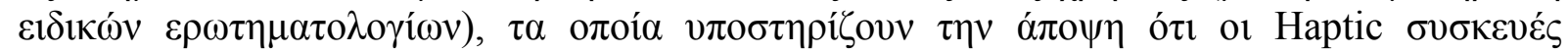

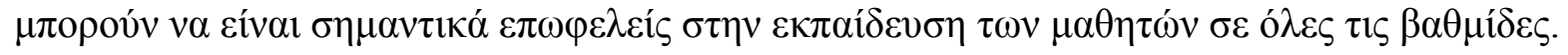

\section{Introduction to Haptics, 3D Graphics and 3D Sound}

Initially, it is useful to define some terms of Haptics, $3 D$ Graphics and $3 D$ Sound, since most haptic devices use this kind of technology.

The term haptics was popularized in the United States by the philosopher Gilles Deleuze and comes from the Greek verb "haptesthai", which means to touch and to handle. The technology refers to the ability of touching and handling virtual objects. In a more general sense, it is an interface that enables users to understand the weight, the shape, the volume of an object and the forces acting on it. By using special input/output devices (joysticks, data gloves, or other devices), users can receive feedback from computer applications in the form of sensations felt on the fingers, the hand, or other parts of the body. The term "tactile feedback" refers only to the sensory input on the fingertips of the user.

With $3 D$ (three-dimensional) graphics we refer to a space where objects (polygons) are made up by a series of dots which are referred to as corners. The coordinates of these corners are specified by three values: $\mathrm{x}, \mathrm{y}$ and $\mathrm{z}$. The representation of the 3D space on each of user's eyes is always a $2 \mathrm{D}$ image obtained through the rendering process. The impression of $3 \mathrm{D}$ is created in the human mind after some (quite elaborate) computation, albeit without conscious effort from the part of the user. Many parameters are used as input to this computation, like the different 2D image in each eye, different colouring (hue) of objects as distance increases, common assumptions as to the size and speed of various objects etc.

As far as $3 D$ sound is concerned, true 3D sound has genuine depth and width to it. Just like 3D graphics, 3D sound can also be recreated by just two speakers and some very advanced mathematics! The use of 3D graphics and 3D sound combined with haptic feedback create a multi-sensory immersion for the users.

\section{MUVII project description}

MUVII stands for the Multi User Virtual Interactive Interface and is the acronym of the IST Project IST-2000-28463. The key objective of MUVII project (WWW) was to develop on the one hand two new Man-Machine-Interface Devices featuring haptic feedback, called Haptic3D-Interface (H3DI), and on the other hand a prototype of an innovative integrated platform 
using the device: the Interactive Kiosk Demonstrator (IKD). University of Patras (HPCLab High Performance Information Systems Laboratory) was responsible for the design and integration of the IKD platform as well as the development of the 3D haptic applications. In cooperation with The Science Laboratory of the School of Education of University of Patras, provided the specifications of the device and carried out the testing of the whole platform with pupils and teachers. The other partners of the project were: Laval Mayenne Technopole (France), CEA - Commissariat a l'Energie Atomique (France), SINTEF - The Foundation for Scientific and Industrial Research at the Norwegian Institute of Technology (Norway), De Pinxi (Belgium), Institut für Kommunikationsakustik - Ruhr University of Bochum (Germany), ONDIM (France), CompuTouch (Norway), Centre PIC (Russia).

Nowadays, educational content and applications are developed everywhere. The use of webbased applications to institutions and universities of higher education has been extensively used, especially on distance education universities, like Hellenic Open University (Papadakis et. al., 2005). However, when talking about teaching in primary and secondary schools the opportunity of having a natural "look and feel" environment for teaching purposes is very promising indeed.

The purpose of MUVII IKD was to demonstrate new interactive paradigms in a novel integration of the following modalities as these interfaced interactively with the user: $3 D$ vision, 3D-audio and haptic (force and tactile) feedback. The process followed in order to design, implement and test the IKD was:

1. User requirements and constraints for the IKD device and applications were gathered and analyzed.

2. Technically feasible specifications of the IKD device, applications and platform were defined in detail.

3. Design and development of the IKD device

4. Design of the modular architecture of IKD supporting platform.

5. Design and development of IKD Applications.

6. Integration of the hardware and software modules.

7. Educational Testing of the IKD, for more than three months, with an adequate sample of more than 300 pupils, and some teachers.

In the present paper the first two steps of this process are described, and some conclusions regarding the issues raised in them are drawn, which can be used as a useful guide for those interested in developing haptic interfaces and applications for educational purposes.

\section{User Requirements Gathering}

In order to collect the most important user requirements, several discussions with potential users (mostly with teachers and to a lesser extent with students) were held. It is interesting to note that although students normally have a better knowledge of computers than their teachers, they are also "dreamers" and ask for features that are not feasible yet.

During these discussions the concepts behind the current haptic technologies (force and tactile feedback) as well as our ideas were explained. In order to judge the pupils' reaction towards virtual reality environments involving haptic feedback interfaces, some trials were performed using both children (of various ages) as well as adults, and a setup involving commercial haptic interface devices (like Phantom by SensAble Technologies and I-Feel-Mouse by Logitech) was utilised. The results of those trials were most encouraging, especially 
considering that these devices have a "feel" a lot less natural than the one expected from the H3DI of the MUVII IKD.

Moreover, regarding the tactile feedback, it was demonstrated that the tactile motors of CompuTouch (one of the partners in MUVII project) gave the sensory input expected when they were integrated in a common mouse. The users got feedback on their index while interacting with a windows application and the capabilities and limitations of such tactile motors were studied in detail.

The teachers were fascinated with the idea of using haptics in their classes since this technology gives the opportunity to observe, test and simulate phenomena that could not be performed in a class or in a school laboratory (Ioannidis and Garyfallidou, 2001). This is due to several reasons such as safety problems (e.g. it is too risky to use explosives or certain chemicals in an ordinary lab) or too difficult and perhaps impossible experiments ("switch off" friction for instance, driving any type of vehicle and feel the forces of a collision, construct a certain machine etc.).

One of the primary interests during this phase was to specify the best shape and functionalities of the IKD device. The main requirements for the IKD device was the movement independence, the feeling of force feedback independently on each finger, the precision of the movement so that the haptic device could function "transparently" as an extension of the user's hand. Apart from that, users expressed their interest in the use of two devices, one for each hand. Moreover, users wanted a large workspace (i.e. actual space where the device would be active).

As far as the potential applications were concerned, the users described the most educationally preferable software as one that used a scenario that could not be easily performed in a class, but with a high educational value nevertheless, while being exciting enough to attract the student's attention. Many different ideas for educational scenarios were put forward and were exhaustively discussed. Finally two applications were selected and implemented for the IKD, as described in a following section.

\section{User Requirements Analysis}

In the ensuing analysis, it was revealed that as far as the device was concerned, users wanted to use advanced wearable (not ground-based) haptic interfaces instead of joysticks. The characteristics most users required involved grasping, manipulating and throwing objects in the virtual space, while feeling forces and tactile feedback on as many fingers as possible (but at least on the two essential ones: thumb and index). The users required to be able to investigate and explore various $3 \mathrm{D}$ objects and feel their material, surface, size, shape, etc. Another very important characteristic, for the educational use of the IKD was deemed to be its realism, (something that is normally ignored by game developers). Special emphasis was given in support of accurate hand and finger movement.

Considering the projected educational use of the device, users wanted an "easy-to-use" device that did not require in-depth knowledge of computers, robotics, or physics. Another important factor was the weight of the device - the need to be as light as possible so that young children can handle it - and the freedom of movements. The users wanted the device to be a "natural" continuation of their hand, which they can freely move and operate in the application's environment. 
Regarding $3 D$ sound features, users found very interesting the idea of hearing the various sound cues of the application and being able to easily perceive their direction, distance and volume, while at the same time they can communicate with the other users by using open air headphones. Furthermore, Haptic related sounds (i.e. sounds produced directly from the interaction of the user's virtual hand with the objects) should also be supported by the sound subsystem of the IKD.

One of the most challenging requirements was to support two users interacting simultaneously in the context of the same application. The users should be able to jointly manage the common viewpoint, but each user should be able to independently move inside the 3D world of the application. The two users should be able to act simultaneously either on the same or on different objects.

The analysis of the user requirements led to the specifications of the IKD haptic device, the IKD platform and the IKD applications, as described in the next sections.

\section{IKD Haptic Device Specifications}

The MUVII IKD Haptic 3D Interface (H3DI) is the human-interface part of the IKD platform and plays an extremely important role in the functionality of the whole system. The IKD H3DI should respect some specific constraints, regarding the various ages of the final users (children as well as adults), and some requirements that are determined by the IKD applications. The analysis of these requirements and constraints led to the following specifications:

Size of H3DI: How small should the body of the H3DI be? The main concern is the use of the device by young children (primary school). As the size of their hand is smaller, we cannot expect young children to be able to handle with ease (or at all) a device created with a fullygrown male in mind. Therefore, the best possible solution is to create a device that is adjustable in size, so it can be used by adults and children as well. This could be achieved by having detachable pads attached to the main body.

Weight: It should be as light as possible, approximately 100 - 150 grams. The balance of this weight is of considerable importance, as it should not be very top - heavy, or in anyway out of balance. A top-heavy H3DI would feel unnatural to use and, therefore, the user would find it hard to "immerse" in the use of it.

Priorities to be kept in the design: The balance and fit are probably more important parameters than size and weight. Any inevitable compromises made should keep this in mind.

Type of movements: The users should have the capacity to move their hand (with the device worn) freely in space without strict movement restrictions. Moreover, the users should be able to use at least two of their fingers (thumb and index) independently. This way, it was observed that they could easily manipulate virtual objects (albeit in a rather unnatural fashion). Taking into consideration that in this trial a new haptic interface was evolved, this is acceptable. It also turned out that the user could easily ajust him/herself to this situation, e.g. the use of only two fingers to manipulate objects). Furthermore, the precision of the movement is an important factor, because it allows the user to perceive the haptic device as an extension of his hand.

Force feedback: Users should be able to manipulate objects using their two fingers and feel forces acting on them, independently on each finger. 
Tactile feedback: Users should be able to get tactile feedback (feel the surface contours and textures) on their two fingertips. For the MUVII IKD H3DI this tactile feedback is provided by two tactile motors integrated on the H3DI. These motors (see Figure 1) were developed by CompuTouch, a MUVII partner. The integration on the H3DI allows good force feedback on finger, while keeping fingertips free for tactile feedback.
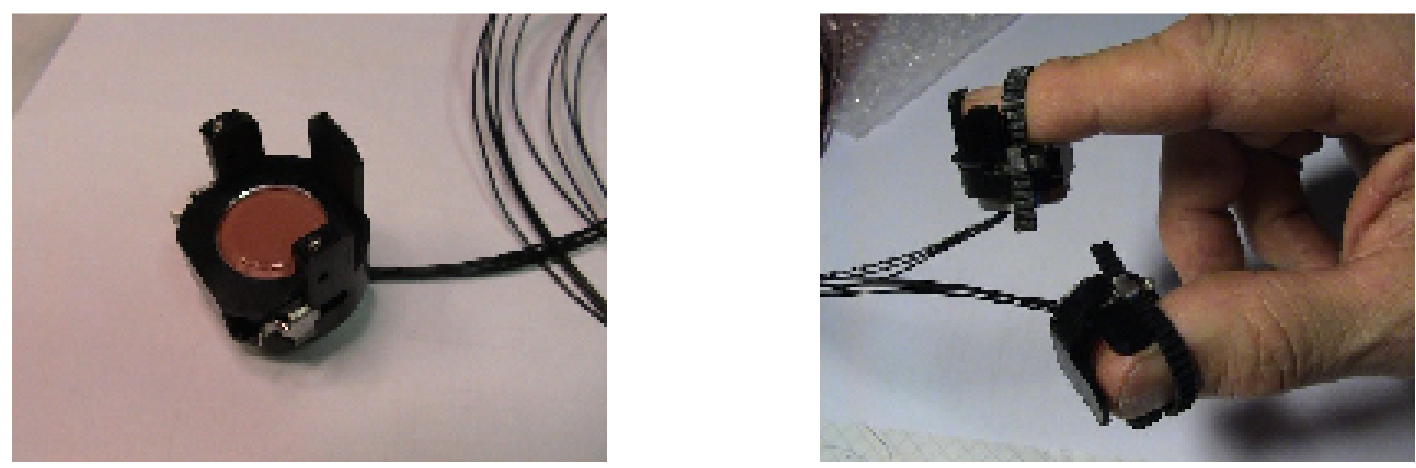

Figure 1: Tactile Motors (Courtesy of CompuTouch $(W W W)$ )

Type of actions: By exploiting the force-feedback of the H3DI, users should be able to select, pick-up, hold, move, orient, release/place, pull/push and throw objects, while they feel forces acting on their fingers (weight, torque, collisions, etc.). By exploiting the tactile feedback users should be able to investigate and explore various 3D objects and feel their surface material, shape, etc.

Workspace: This is the actual physical space where the device would be operational (its position can be tracked). Minimum requirements are $600 \mathrm{~mm}$ (width), $600 \mathrm{~mm}$ (length), and $400 \mathrm{~mm}$ (height). Obviously, the greater the final workspace is, the better for the users.

Robustness: A certain amount of robustness is required especially as we envisage children using it. Ideally, it should be able to withstand a drop from $80-100 \mathrm{~cm}$ height onto a hard floor without breaking or needing adjustment or realignment.

\section{IKD Platform Specifications}

Three modalities were involved in MUVII interactive environment: visual, haptic, and aural (i.e. sound). To achieve the best virtual reality immersion the best solutions for each one of these three modalities involved were selected and (most importantly) integrated together. Indeed, MUVII IKD demonstrated new interaction paradigms and a novel integration of these three modalities: 3D-vision, 3D-audio, and haptic (force, torque, and tactile) feedback.

Innovations of IKD included:

- The multimodality of haptics, 3D-audio and 3D-graphics, to provide an integrated, natural "look and feel" immersion environment for edutainment purposes.

- Design and implementation of a special haptic feedback device (see Figure 2) that support tactile \& 3DOF force feedback, especially designed for educational virtual environments.

- An extensible and modular architecture of the platform that can support the integration of two such haptic feedback devices, thus providing multi-user ability (either teacher-pupil or pupil-pupil) to enhance the teaching procedure and the collaboration among pupils.

- Support for motion capture / tracking for hand and head of two users.

- Sophisticated 3D-sound: use of open headphones, head-tracking and real-time reproduction of individual 3D sound for each user. 
- Innovative haptic interaction metaphors, like the combination of visual with haptic or audio with haptic stimuli, aiming at the creation of a multi-sense educational environment. These metaphors give emphasis on events that cause haptic and tactile feedback to the users, mainly through "real-world" and "non-real-world" audio cues, sound effects and visual effects.

- Rapid application development support through the integration and customization of a commercial tool (Virtools), the MUVII IKD library of predefined generic objects \& behaviours and the MUVII IKD audio authoring toolkit.

- Innovative Educational Demo Applications: These applications introduce several innovative features and their primary purpose is to demonstrate the capabilities of the H3DI and to build on its functionality, aiming at the rapid adaptation of users in the characteristics (and in the way of use) of the device.
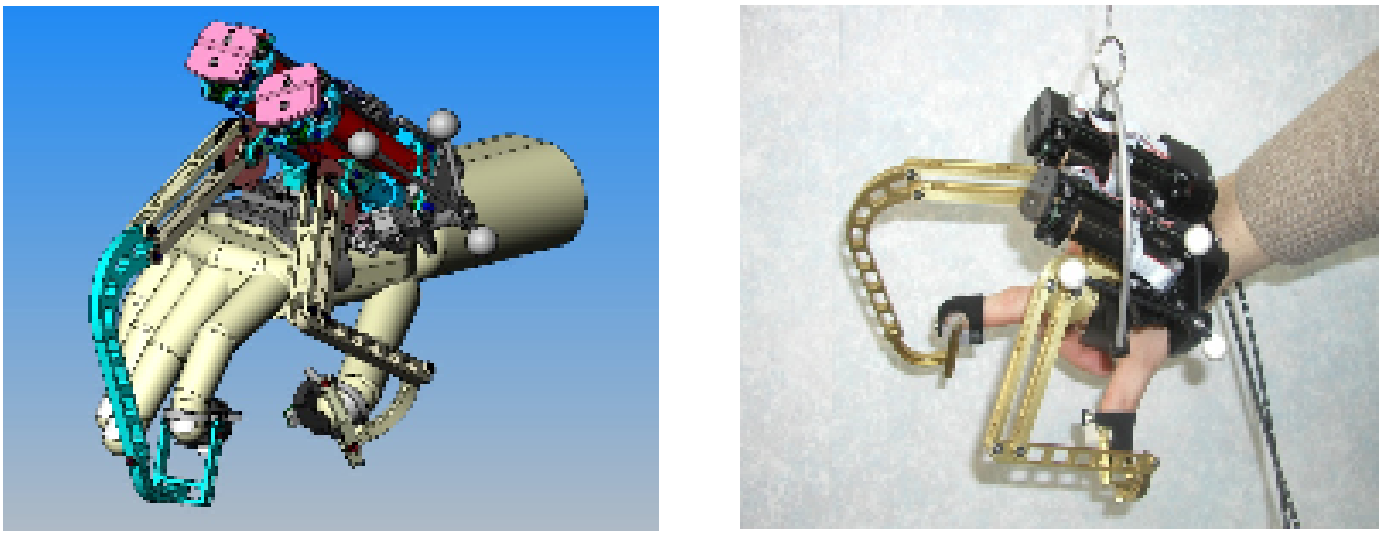

Figure 2: IKD H3DI concept and realization respectively (Courtesy of CEA (WWW))

Figure 3 shows the hardware architecture of the IKD, showing PC hosts in charge of each module and network communication connecting these hosts.

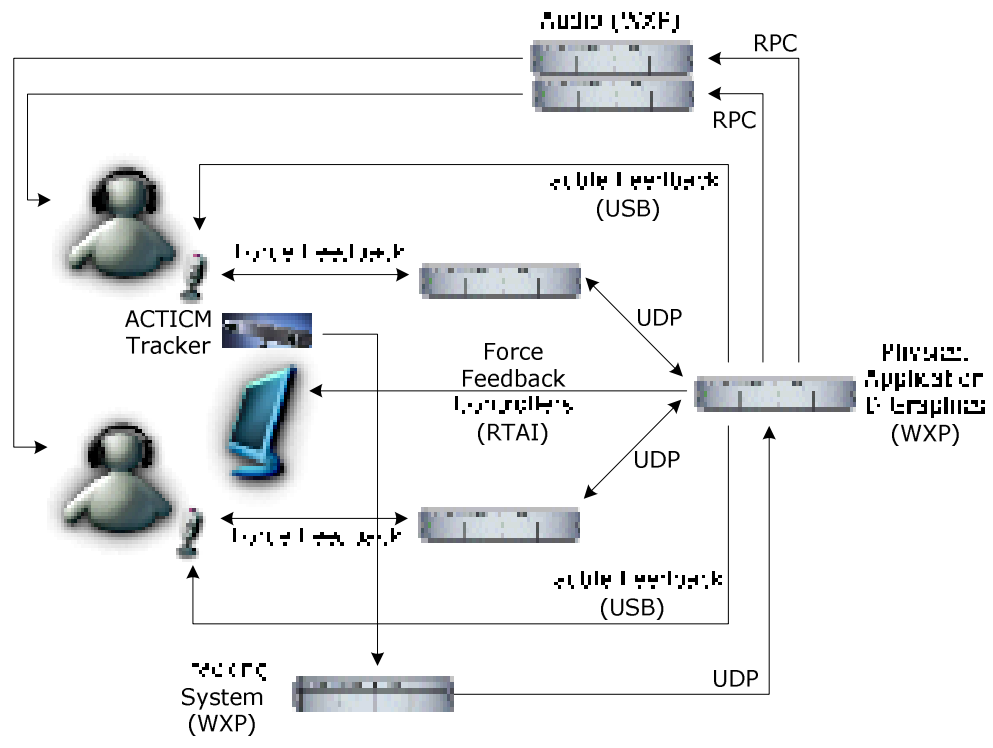

Figure 3: IKD hardware architecture

The really hard and technically challenging issue for IKD was to keep the latency time between visual, haptic, tactile and audio feedback as small as possible in order to achieve a great degree of realism. Very short time latency was also required for the hand tracker that 
was used for the accurate positioning of the real human hand and the real-time rendering of the virtual hand inside the 3D world of the application. The problem is getting harder, if you consider that these subsystems of the IKD platform are implemented by different pieces of software which are being executed in different computers and in some cases under different operating systems all connected to form a single system.

\section{Haptics and Education}

In a conventional class environment, when instructing a pupil on the way to achieve a manual task, the teacher will occasionally resort to demonstration through a direct mechanical contact (especially so for younger pupils). Unlike visual demonstration or verbal instruction, mechanical or haptic demonstration is designed to communicate directly to the pupil's hand. Common examples of this kind of teaching occur in sports and music instruction.

Traditionally most of our daily activities were accomplished by the use of the human hand. Computers may have brought significant changes to our daily life but the interface is still carried out through a keyboard and a mouse which are data input devices, that do not offer to the user any information related to the "object" he/she manipulates, and this is equally so even if we speak about virtual reality. Computer never sends data to the keyboard or the mouse because these could not create haptic reaction to the user's hand. Haptic devices come to eliminate this limitation.

"Unlike traditional interfaces that provide visual and auditory information, haptic interfaces generate mechanical signals that stimulate human kinesthetic and touch channels. Haptic interfaces also provide humans with the means to act on their environment." (Hayward et al, 2004).

But could haptics extend the teaching possibilities, esp. for science concepts?

Some research groups like NanoScale Science Education Research Group (WWW), investigate the impact of haptics on students' learning of science concepts, and their results so far are very encouraging. According to their research, when a student uses haptic devices receives two kinds of stimulants distinguished in kinesthetic and tactile perception. Both stimulants are dynamic, which means that they change as the user is operating the objects, no matter if they are physical or virtual. This active touch involves intentional actions that an individual chooses to do, whereas passive touch can occur without any initiating action. For educators, involving students in consciously choosing to investigate the properties of an object is a powerful motivator and increases attention to learning.

Moreover, it is experimentally proved by Sathian et al (1997) and Itan (2005) that where vision is the dominating sense in form perception (macro-geometry) of bodies, touch excels in defining their texture (micro-geometry).

Other researchers (Okamura et al, 2002) showed that a purely audio-visual environment, even if it is highly interactive, can present difficulties for "haptic learners". By addressing the sense of touch, haptic interfaces are promising tools for helping students with haptic cognitive styles obtain an understanding of mathematical and physical phenomena. 


\section{IKD Educational Applications}

Haptic technologies offer an enormous variety of applications starting from "virtual" school laboratories and going as far as learning auto driving, surgery, or even space ship manoeuvres. In such a virtual reality environment that supports haptics, the applications could provide the users with the capability to:

- investigate and explore various 3D objects. (Tactile feedback, which involves Material, Surface of objects, Size, Shape, Weight etc.)

- feel force and torque feedback (caused by weight, torque etc)

- select, pick-up, hold, move, orient and release/place objects

- hear sounds that emulate sounds produced by surfaces rubbing against each other or by surface-collision events etc.

The educational applications that were developed in the framework of MUVII project were built to exploit the advanced features of MUVII IKD platform and device and to investigate their educational potential. Indeed, the didactical opportunities offered by the system (as it gradually developed) were painstakingly analyzed and evaluated. Subsequently, two educational applications were selected for implementation in the IKD: (A) Newtonian Physics, Trajectories and the Solar System, and (B) Virtual Model Assembly - Gears (each one with learning mode, recapitulation mode and edutainment mode).

From the educational point of view, both educational applications were based on the constructivist theory (for an overview of the theory see Ioannidis et al, 2005, section 3.5). The basis of the constructivist theory of learning and instruction is formed by the writings of Piaget (Piaget, 1972 and Piaget et al 1995) and Vygotsky (1978), along with the work of Ausubel (1968) and Bruner (1990). According to this theory students are responsible for their own learning, and meaningful learning demands that pupils construct their own knowledge.

Computer aided learning and virtual reality environments allow students to learn by following his/her own pace, or even according to their interest. Using the MUVII IKD educational applications in their "active manipulation" mode, students can manipulate objects after consciously deciding to do so. Thus, users interact with the objects they choose in the way they choose, and feel the feedback from their actions. This stimulates their interest and increases their attention. Our study showed that the knowledge remaining in the student's mind after such a learning activity is higher than what is left after teaching the same subject using traditional methods of teaching, where the student passively listens to the teacher teach or watch a science video.

Within the application of Newtonian Physics and the solar system, the user interactively (and virtually) navigates through the solar system, while collecting information about anything that interests him/her. The user experiences the effect of the forces when accelerating objects (e.g. by trying to throw them off their course) as well as the strength of the gravitational forces applied to objects at different distances from the sun or from a certain planet. Obviously, for the purpose of such an interaction the user is endowed with "super-powers". With the use of haptics the pupils are able to experience, feel and gradually learn the way the laws of simple mechanics in the way these are applied at the scale of our solar system. Figure 4 shows a screenshot of the application. 
Regarding the Virtual Model Assembly - Gears, the user is offered a lesson of the history of cogs, gears and their applications through the ages. They can also try to assemble some selected applications by combining gears. The users experience the effect of forces like those caused by weight, friction, motion, rotation etc. This application can also be used to enhance students' understanding of phenomena like the transmission of motion from one part of a machine to another. Figures 5 and 6 show two screenshots of this application, the second one using the watermill paradigm.

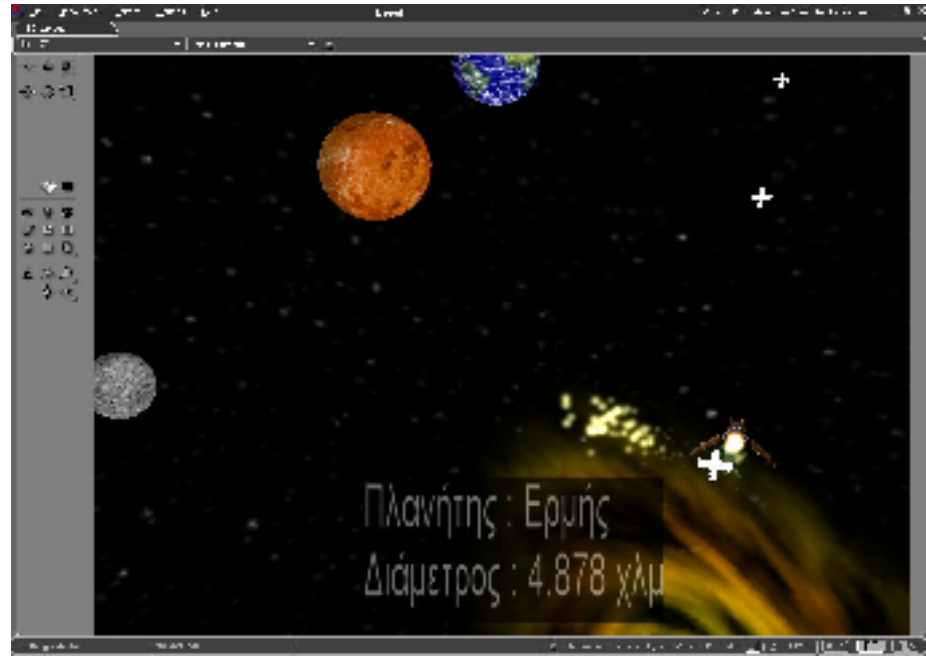

Figure 4: Feeling the gravity (Planet: Hermes Diameter: $4.878 \mathrm{~km}$ )

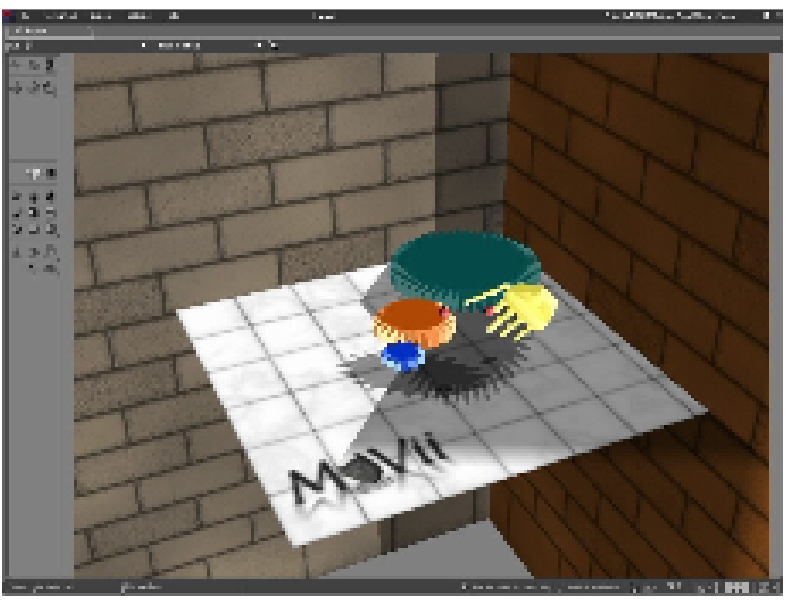

Figure 5: Feeling moving gears

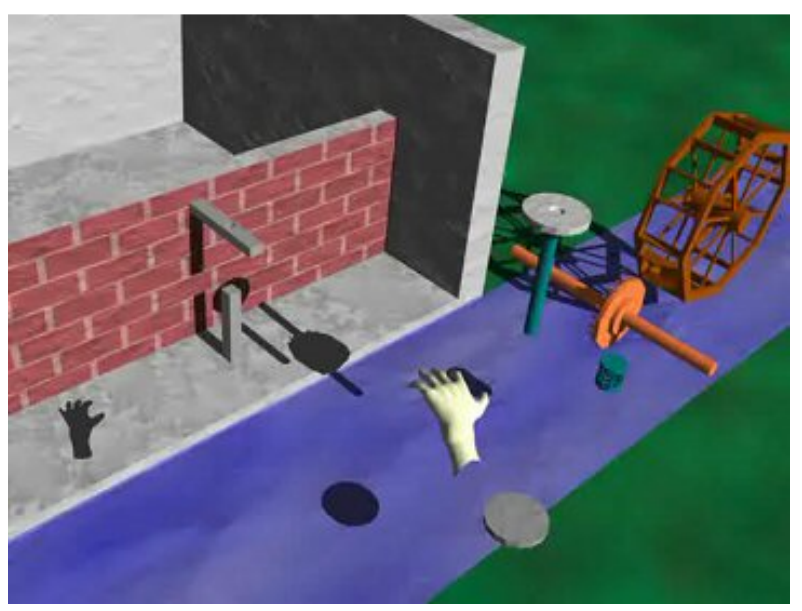

Figure 6: Assembling a windmill

\section{IKD Experimental Validation}

The first public experimental validation of IKD H3DI, software infrastructure and applications, took place in May 2004 in Laval Virtual (see Figure 7 and 8) exhibition event, one of the biggest annual exhibitions in Europe for Virtual Reality.

Despite the fact that the device was aimed at children of ages above 10 years old, it occurred that even younger children (e.g. 7-10 years old) could use it effectively. During the tests in Laval the users were very enthusiastic and attracted by the device and the applications. 
Almost no-one gave up trying to finish the application, despite the difficulties everyone faces when coming in contact with a new technology and a virtual space he/she has to interact with. The testers were invited to write their opinion and impression about the IKD, some of which are presented here:

Kevin, 12:

I find it very good fun, and I think that this system could be used to control/test objects in space or on planets.

Christian, 36 :

Very good sensation of the movements in space and of the grapping. Very realistic force feedback. Thank you

Nicolas, 24:

Feelings almost real, impressive sensation of resistance. I think that many useful applications can be developed.

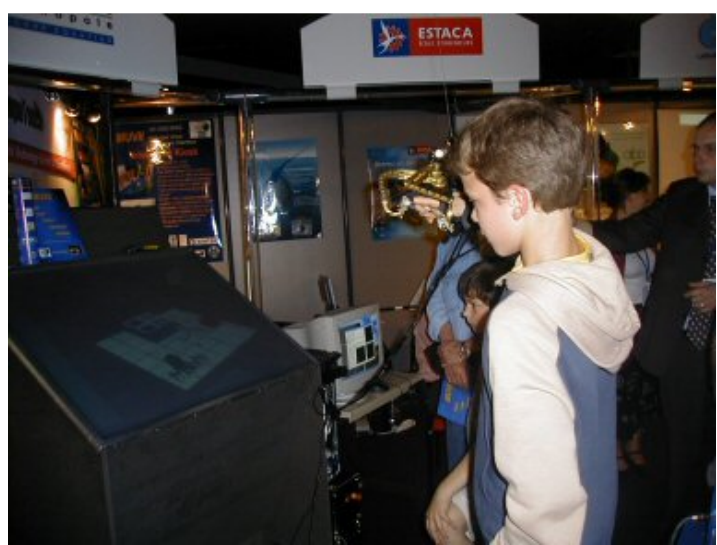

Figure 7: Laval Virtual demonstration, a young user

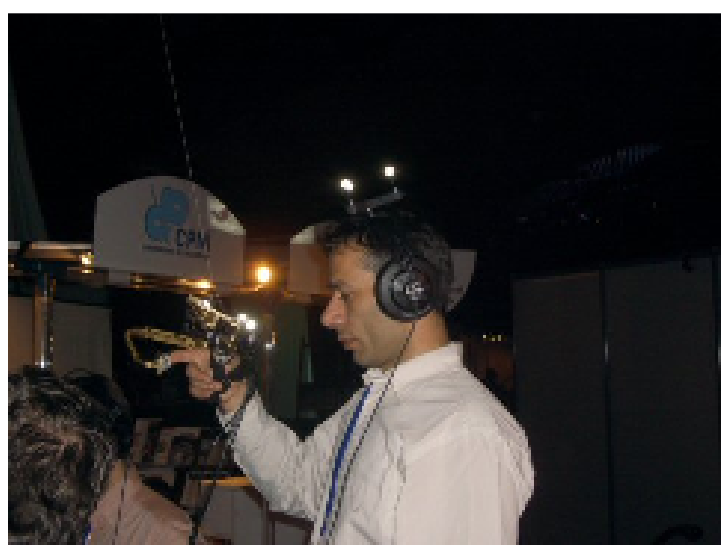

Figure 8: Laval Virtual demonstration, an adult user

After this initial demonstration of IKD, the educational trial of the IKD system took place, for more than three months, with an adequate sample of more than 300 pupils, and some teachers.

IKD H3DI, software infrastructure and applications have been tested, against functional and performance issues, with schoolchildren that have been randomly selected from various schools of Patras and Achaia prefecture. It should be mentioned here that mostly due to the equipment size, and the lack of suitable space in schools, the students that participated in the testing procedure had to visit the device which was set at laboratory at the University of Patras (HPCLab). Members of the Science Laboratory (School of Education) were responsible for the educational trial.

In this educational trial 163 students participated:

64 of which were primary school pupils,

$\mathbf{7 4}$ were lower secondary school students, and

25 were upper secondary (i.e. lyceum) school students. 
For the educational trail all the international accepted practices concerning research in education were followed. The "exercises" for each group of students were chosen in accordance to their age. The educational results of this teaching approach, as well as the feedback derived from the users were presented in (Christodoulou et. al., 2005). We summarise the most important ones below.

- A large proportion of children found teaching with the use of a haptic device interesting. Students responded quite well to the use of this haptic prototype, although this could be made to look "friendlier".

- Students were also pleased and seemed to be even amused by their experience. They would like to repeat the experience they have had and they would also like to see the haptic device extend its abilities; in addition they wanted to see haptics and the whole IKD device in particular being used in other (more varied) applications.

- No student found the experiment boring or too difficult (which is a big compliment!).

- Assessing the learning achieved using the application on the Newton's law on gravity, we observe most students choosing the correct answer most of the time. By comparing these answers with these of the pre-test (a questionnaire filled by students before the trial), an overall increase in understanding is obvious. This really is "understanding by feeling"!

- The great majority of students were interested in the haptic application (as well as the device) one way or another. Younger children by being naturally "enthusiastic" found the curriculum interesting in higher percentages than the older ones.

- The majority of the students were willing to participate in future lessons using devices with haptic interfaces, while a sizable proportion of them would also wished to have had such a device available for their personal use at home.

- It was also observed that generally the girls seemed to consider (e.g. to think about) their hand-movements before they made them, and as a result, their handling of the haptic device was steadier. The boys seemed to be more impulsive (- anxious even) and made quick movements (almost jerky, sometimes). The above are general observations made by the researchers for the bulk of the students, while it should be stressed that individual handling skill differed amongst students.

Overall, the encouraging result from this investigation is that we can easily use IT and haptics to teach science (and perhaps other subjects) even with primary school pupils.

\section{Conclusions}

The multi-sensory environment of a haptic device can greatly improve the existing teaching methods, by offering tools of enhanced quality suitable for deeper understanding of the entities taught. Students seem to have adjusted well to the new system and have enjoyed using it. Currently there are no other applications with capabilities similar to the ones specified in MUVII project, incorporating characteristics like multi-user collaboration among students and teachers, efficient 3D-sound sub-system, targeting children of various ages, efficient haptic interaction metaphors etc.

Some significant requirements and specifications for haptic devices are outlined in this paper, mainly for applications that will be used in education. Those who seek to develop haptic interfaces, as well as application software for education, will find the specifications presented 
extremely useful. In addition to that, this paper will help potential users (teachers, students) become more acquainted with the use of haptic interfaces in education, understand the power (and limitations) of haptically-enhanced virtual reality educational systems, and provide some useful feedback on the prospective implementation in their everyday school practice. 


\section{References}

Ausubel, D. (1968). Educational psychology: A cognitive view. New York: Holt, Rinehart \& Winston. Bruner, J. (1990). Acts of meaning. Cambridge, MA: Harvard University Press

Christodoulou S. P., Garyfallidou D. M., Gavala M. N., Ioannidis G. S., Papatheodorou T. S., and Stathi E. A. (2005), "Haptic devices in Virtual Reality used for Education: Designing and educational testing of an innovative system", Proceedings of International Conference ICL2005 (Interactive Computer Aided Learning) September 28 - 30, 2005 - Villach, Austria

Hayward V., Astley O.R., Cruz-Hernandez M., Grant D. and Robles-de-la-Torre G. (2004) Haptic interfaces and devices. Sensor Review 24(1), Emerald Group Publishing Ltd., ISSN 0260-2288, pp. 16-29

Ioannidis G. S. and Garyfallidou D. M. (2001), Education using information and communication technology (ICT), and ICT education: categories methods and trends, In: Auer M. and Auer U. (Eds.) Proc. ICL2001 workshop: "Interactive Computer aided Learning, Experiences and visions", Villach, Austria, Kassel University Press, (2001) ISBN 3-933146-67-4

Ioannidis G.S., Garyfallidou D.M., and Spiliotopoulou-Papantoniou V. (2005) Streaming Media in Education, and their impact in teaching and learning Educational best practices and some first observations of their implementation. Published by Education Highway, ISBN 3 - 9500247 - 4 - 3, (First Edition, July 2005), also available at http://estream.schule.at/

Itan, Y. (2005) Human motion perception mechanisms: A functional magnetic resonance imaging experiment applying Computational, Psychophysical and Physical methods, available at: http://www.homepages.ucl.ac.uk/ ucbpyit/Essay3PDF.pdf (visited in February 2007).

Okamura A.M., Richard C. and Cutkosky M.R. (2002) Feeling is believing: Using a force-feedback joystick to teach dynamic systems, ASEE Journal of Engineering Education, 92, 3 (Jul 2002), pp. 345-349

Papadakis S., Xenos M., Mitsou E., "Experiences and technical issues from the delivery of computer-based learning materials in the Hellenic Open University", Open Education: the Journal for Open and Distance Education and Educational Technology, Vol. 1, No. 2, pp. 12-28, 2005.

Piaget, J. (1972). The psychology of the child. New York: Basic Books.

Piaget, J., Gruber, H. (Ed.), \& Voneche, J. J. (Ed.) (1995). The essential Piaget Publisher: Jason Aronson; 100th Anniversary Ed

Sathian, K., Zangaladze, A., Hoffman, J. M. and Grafton, S.T. (1997) Feeling with the mind's eye, NeuroReport, 8, (1997), pp. 3877-3881

Vygotsky, L.S. (1978). Mind in society: The development of higher psychological processes. Harvard University Press.

CEA - Commisariat a l'Energie Atomique (WWW), http://www.cea.fr

CompuTouch AS (WWW), http://www.computouch.no/

MUVII Web Site (WWW), IST Project: Multi User Virtual Interactive Interface, http://muvii.hpclab.ceid.upatras.gr, visited in March 2007

Nano-Scale Science Education research group (WWW), College of education, NC State University, http://ced.ncsu.edu/nanoscale/haptics.htm, visited in March 2007 


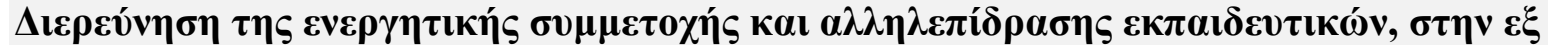

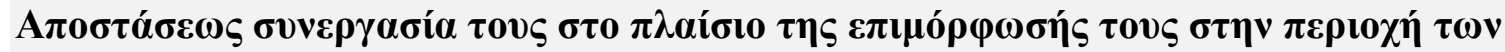

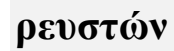

\section{Investigation teachers' active participation and interaction, concerning distance collaboration, during their in service training in fluids}

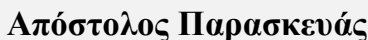

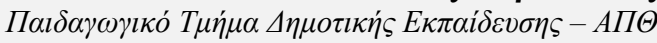

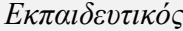 \\ e-mail:aparaske@eled.auth.gr \\ $\Delta \eta \mu \eta ́ \tau \rho \eta \varsigma \Psi v ́ \lambda \lambda o \varsigma$

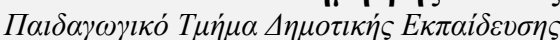 \\ $K \alpha \theta \eta \gamma \eta \tau \dot{\iota} \varsigma \Pi T \Delta E-A \Pi \Theta$ \\ e-mail:psillos@eled.auth.gr
}

\begin{abstract}
Many researchers interest is focused on studying several variables that affect collaborative methodologies which implemented in teachers' distance training programs, technology based. Recently these technology based distance learning programs were implemented with a combination of face to face meetings described as blended learning models.

In the present work we describe the investigation of two variables that appear to influence distance collaboration, with the implementation of Henrys' content analysis model. Those variables were active involvement and interaction among participants. Content analysis model was applied in the last stage of a didactic approach of blended character in practicing teachers in the area of fluids.

Initially it is described the theoretical part, the research methodology, evaluation data from model implementation and concluded with the results and discussion.
\end{abstract}

\section{Пврі́ $\eta \psi \eta$}

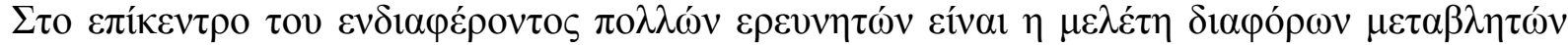

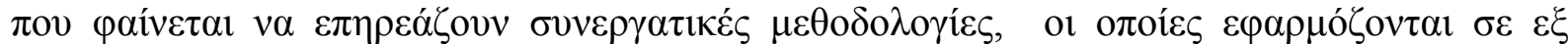

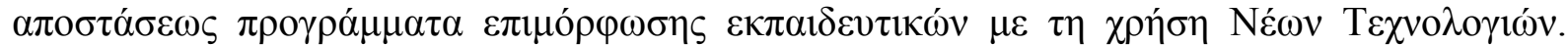

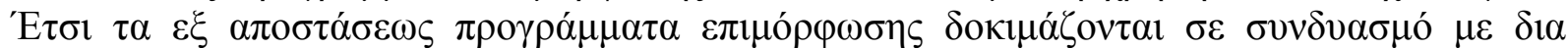

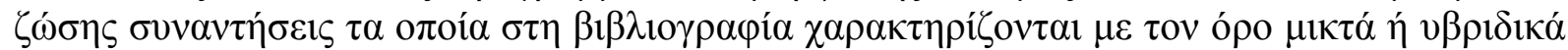
$\mu \mathrm{ov} \tau \dot{\varepsilon} \lambda \alpha \mu \alpha \dot{\theta} \theta \eta \sigma \eta \varsigma$.

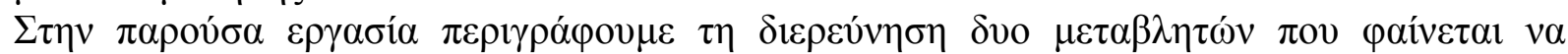

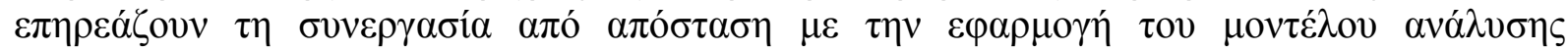

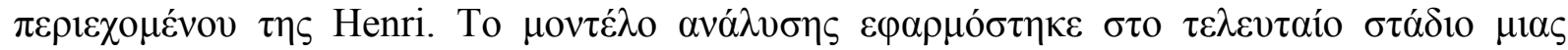

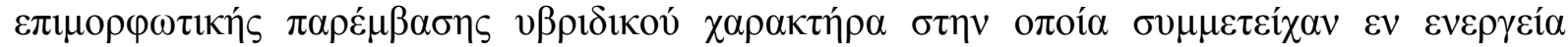

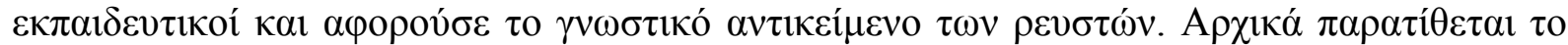

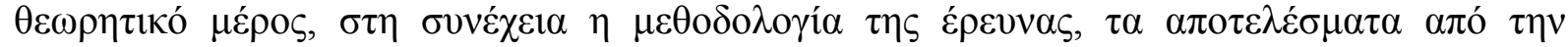

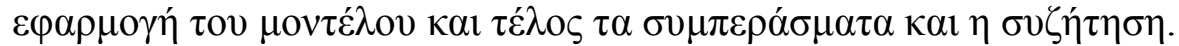

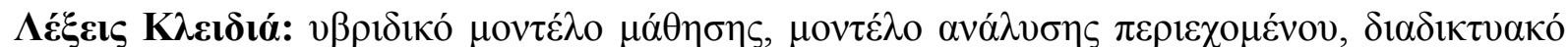

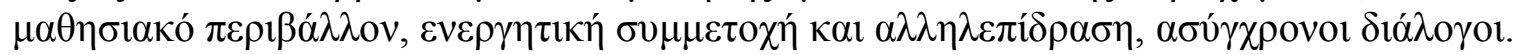




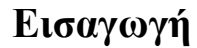

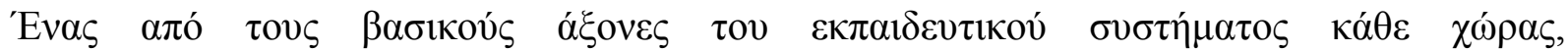

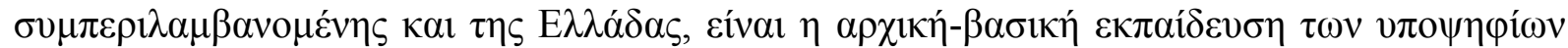

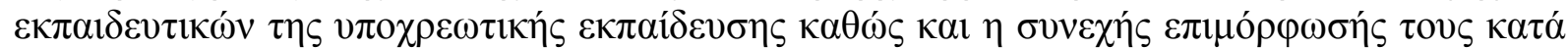

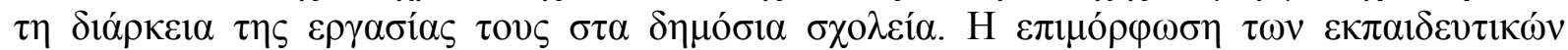

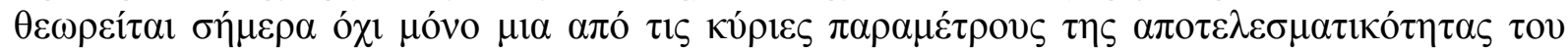

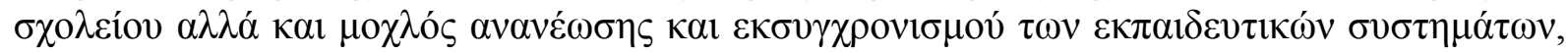

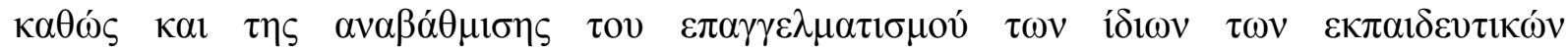

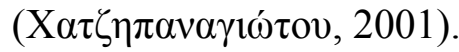

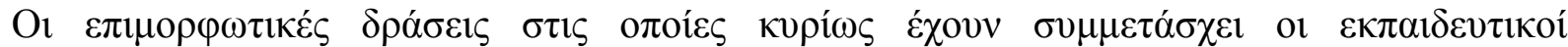

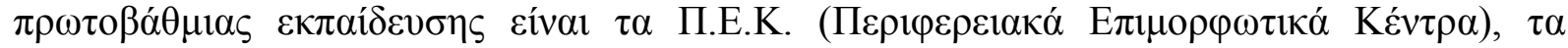

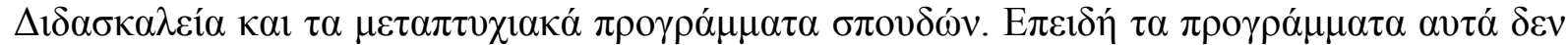

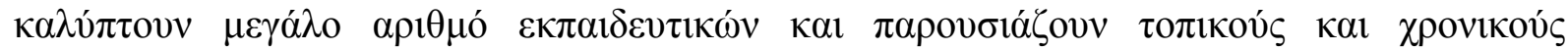

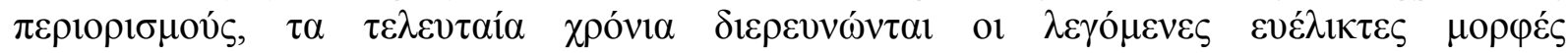

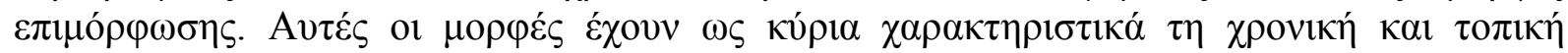

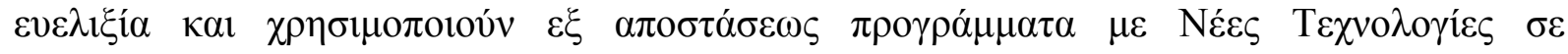

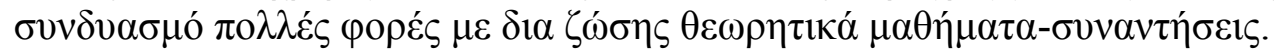

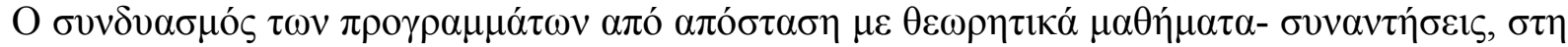

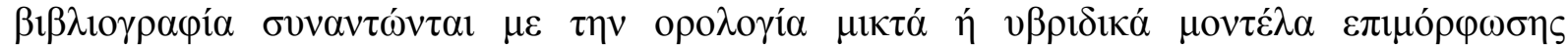

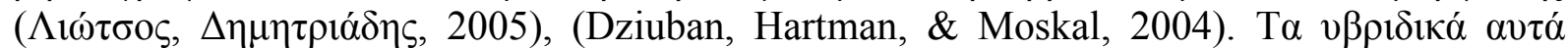

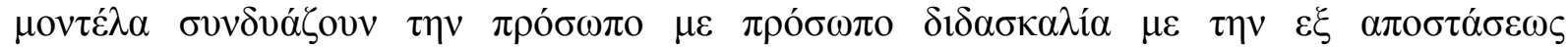

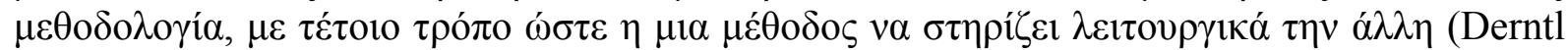

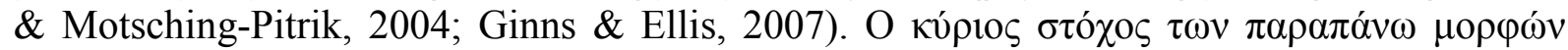

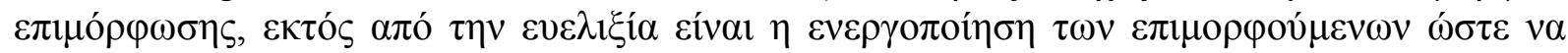

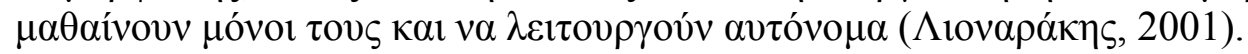

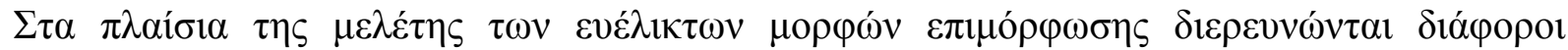

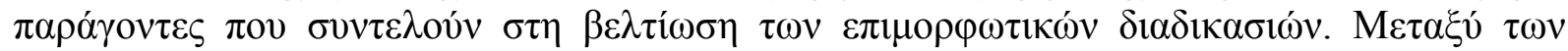

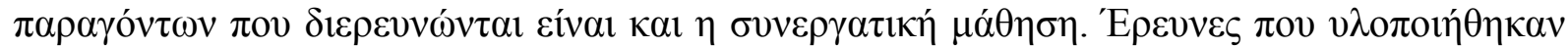

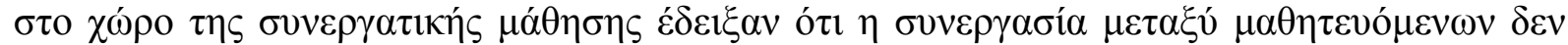

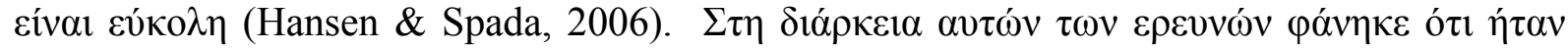

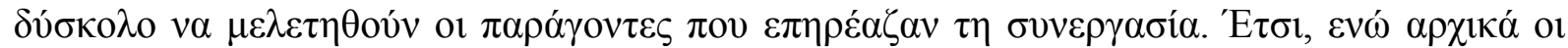

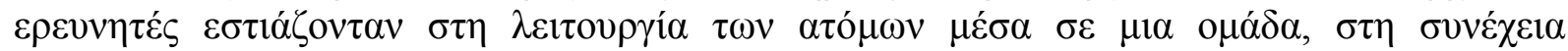

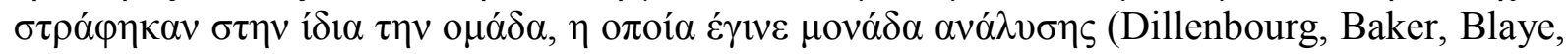

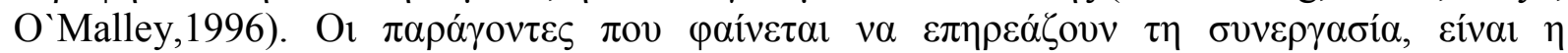

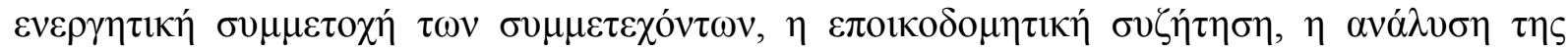

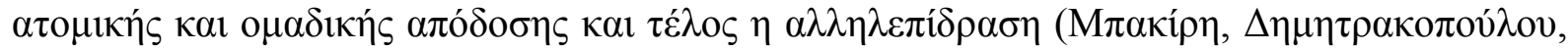
2001).

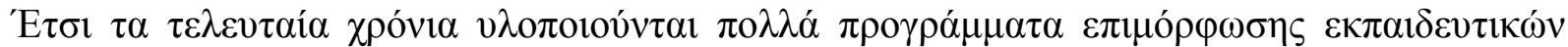

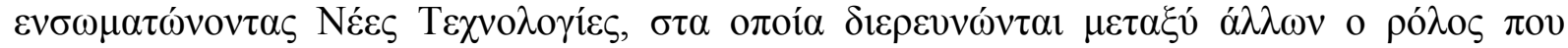

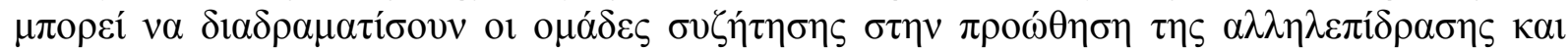

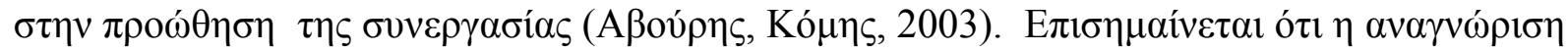

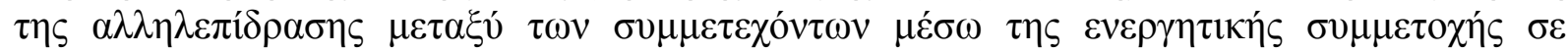

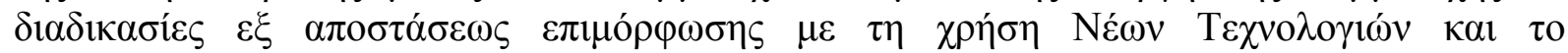




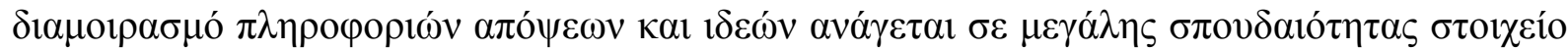
(Harasism, 1989).

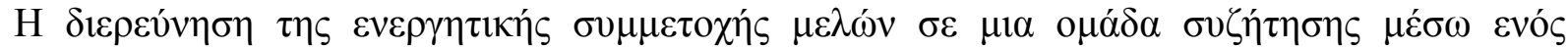

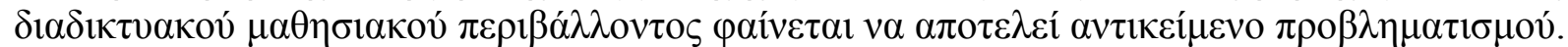

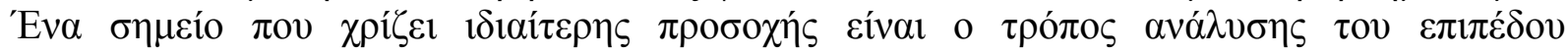

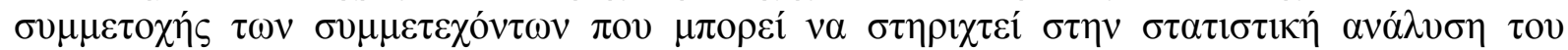

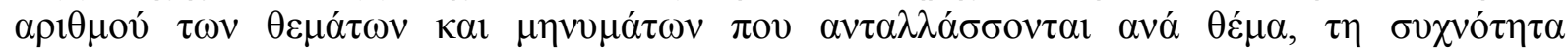

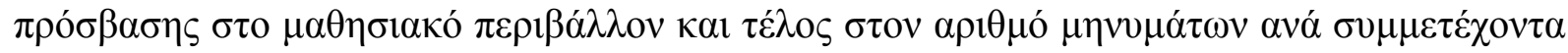
(McKenzie \& Murphy, 2000).

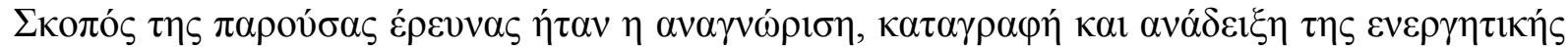

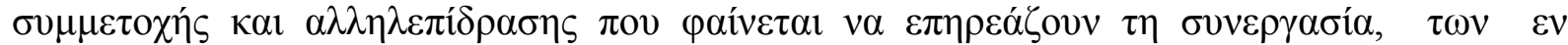

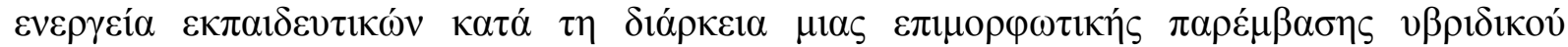
$\chi \alpha \rho \alpha \kappa \tau \eta ́ \rho \alpha \sigma \tau \alpha \rho \varepsilon v \sigma \tau \alpha$.

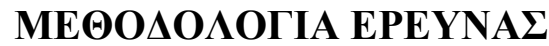

\section{$\Delta \varepsilon i ́ \gamma \mu \alpha$}

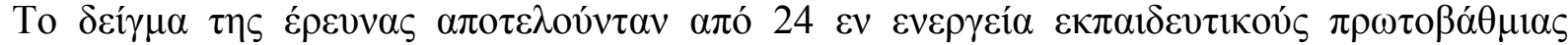

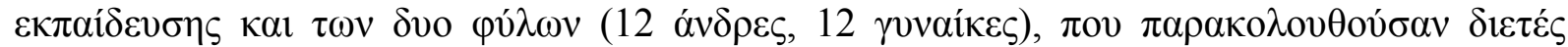

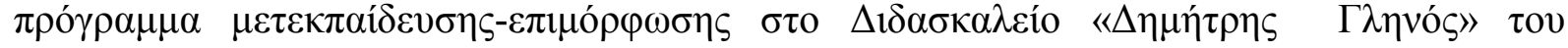

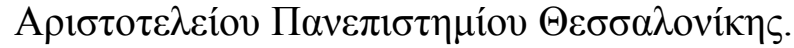

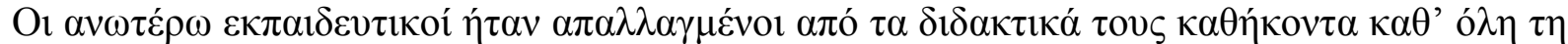

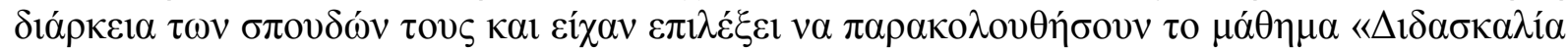

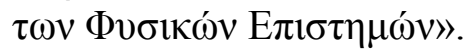

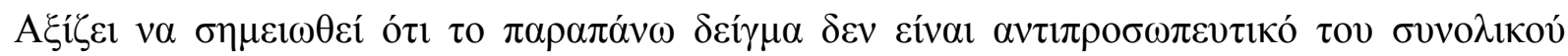

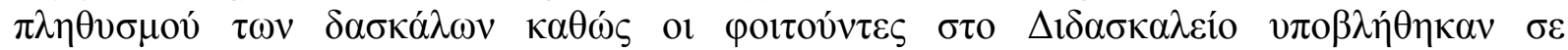

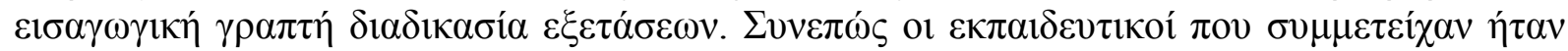

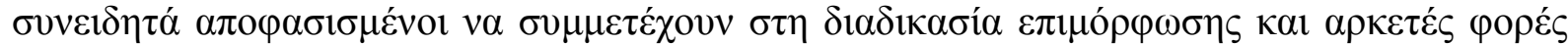

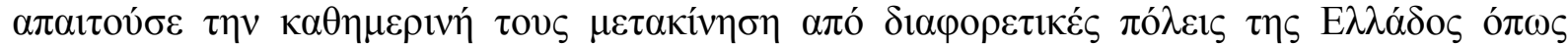

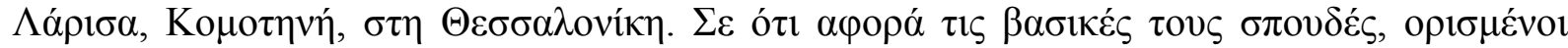

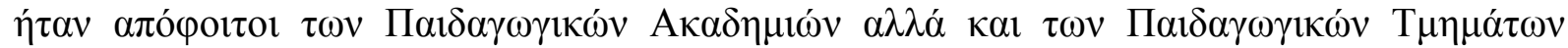

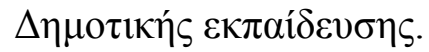

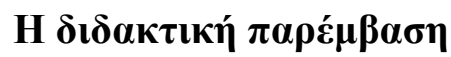

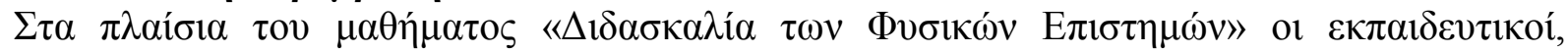

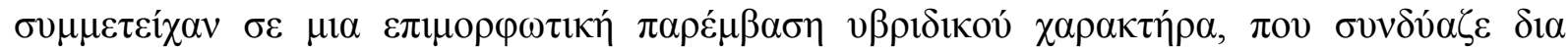

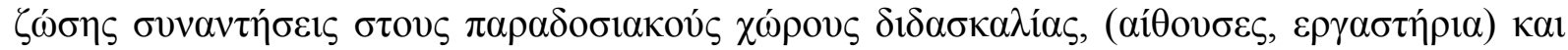

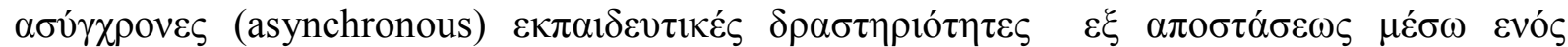

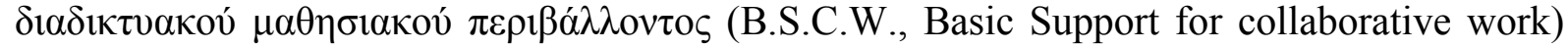
(Paraskevas et al. 2003).

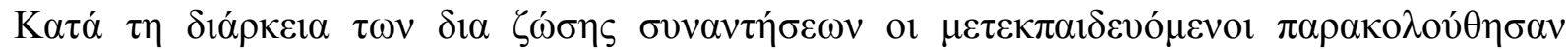

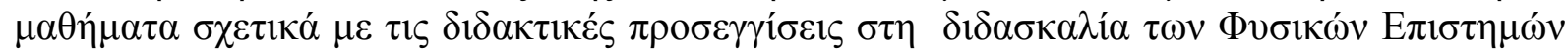

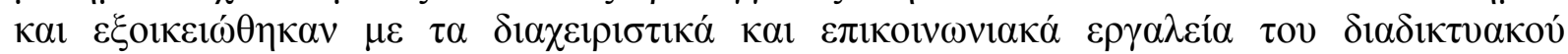

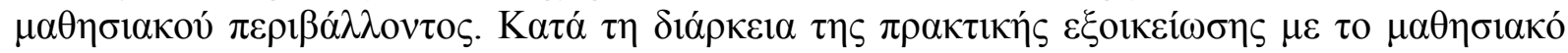

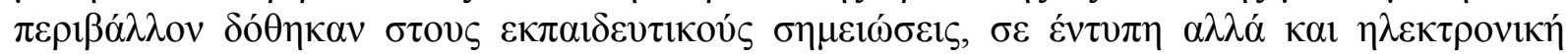




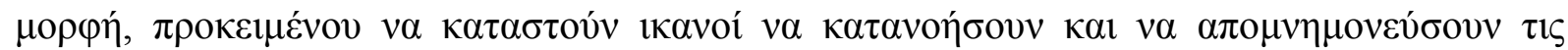

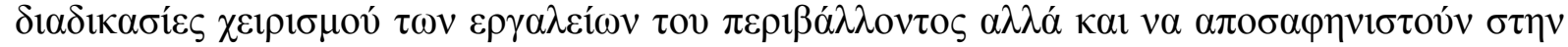

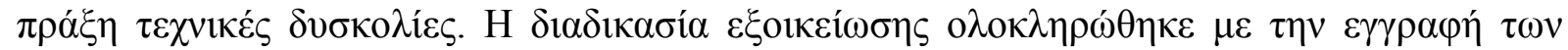

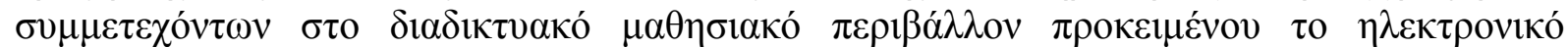

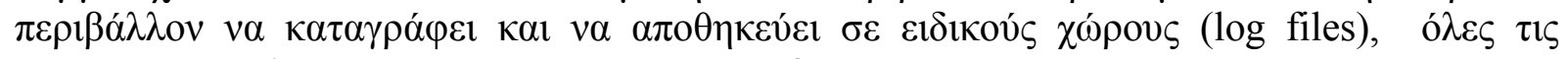

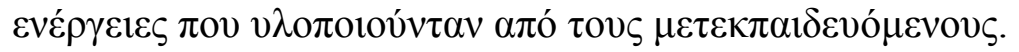

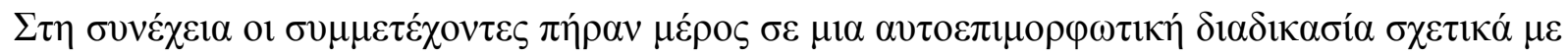

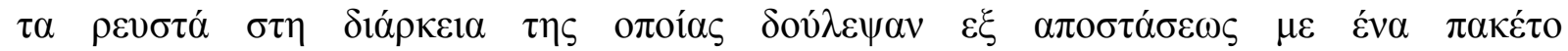

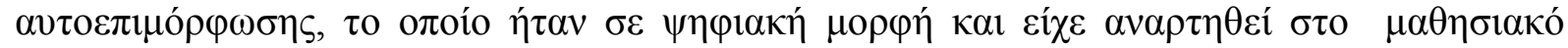

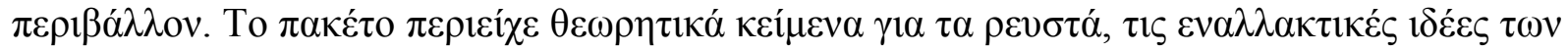

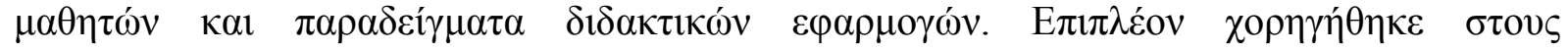

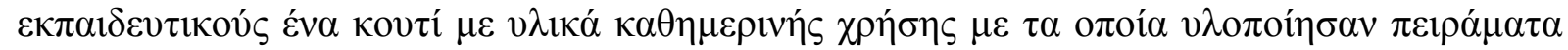

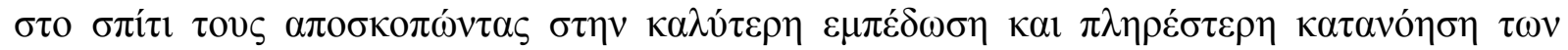

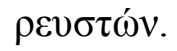

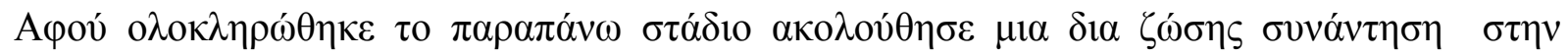

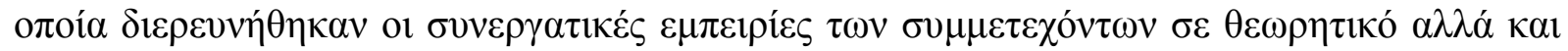

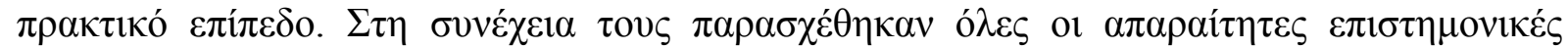

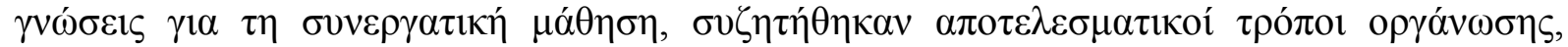

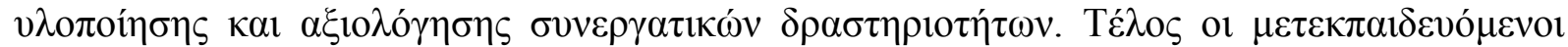

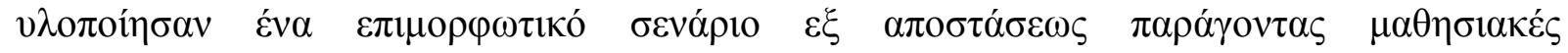

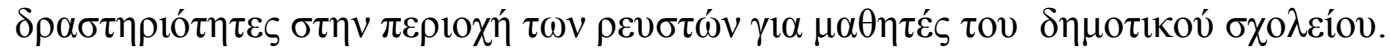

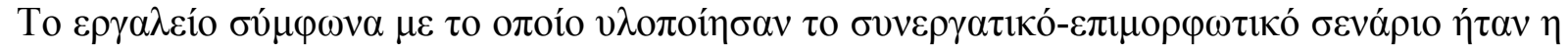

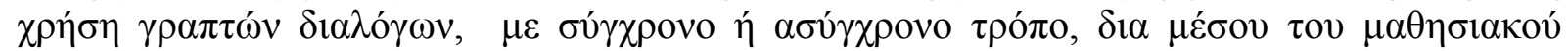

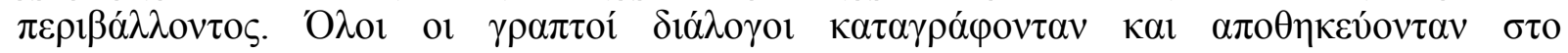

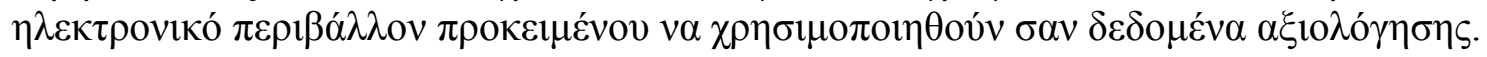

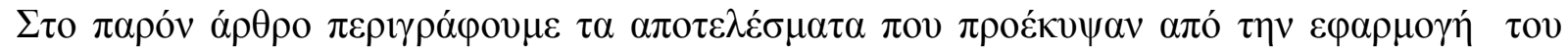

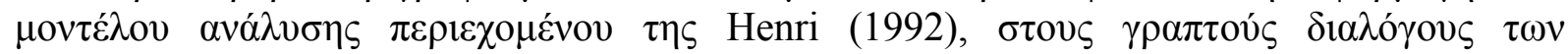

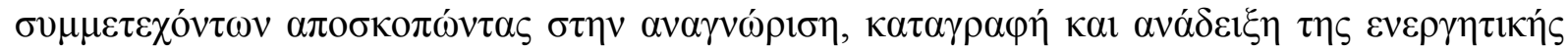

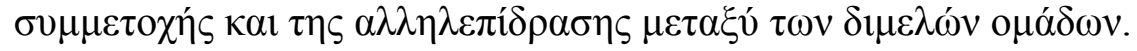

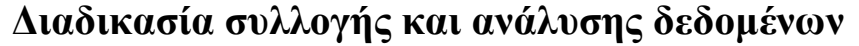

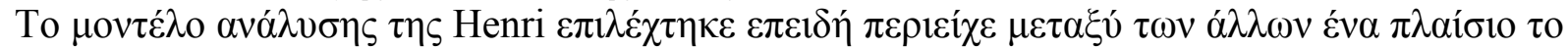

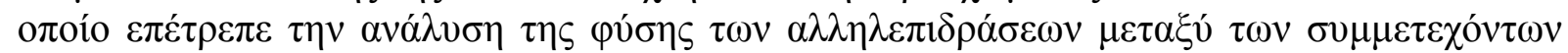

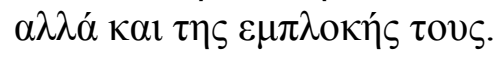

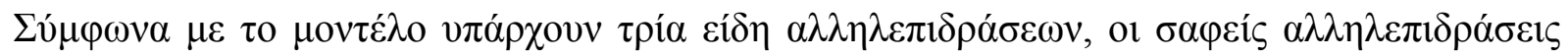

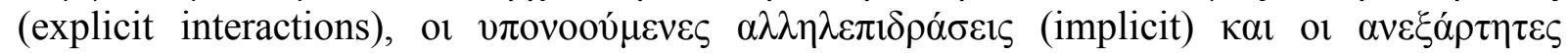

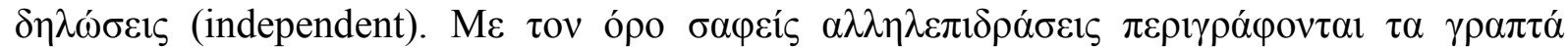

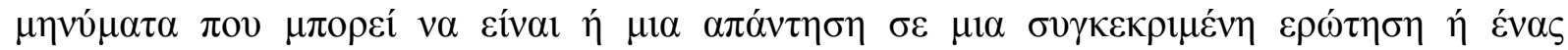

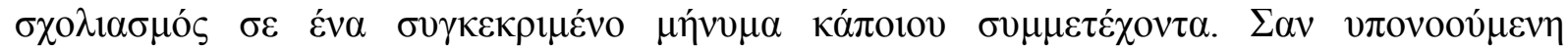

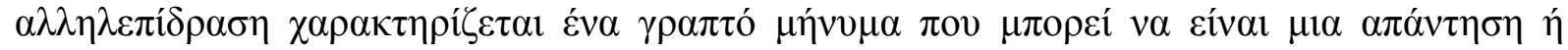

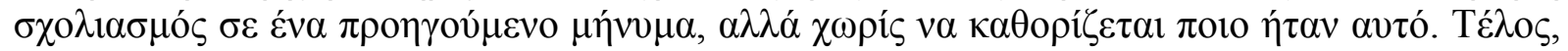

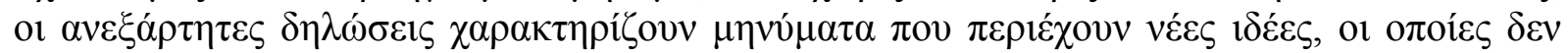

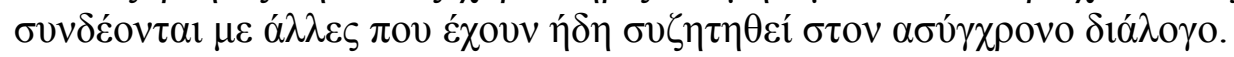

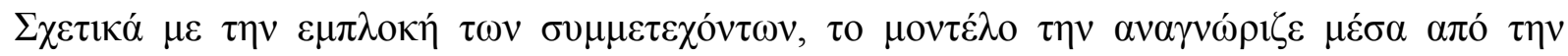

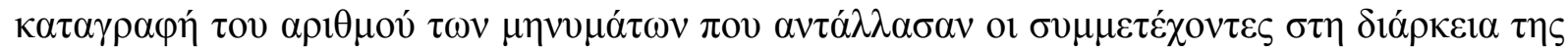

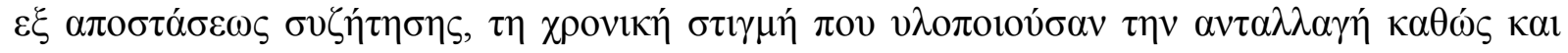




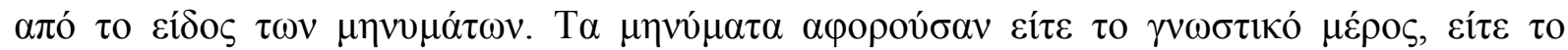

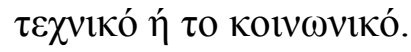

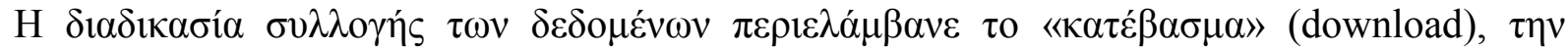

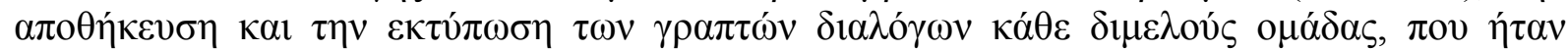

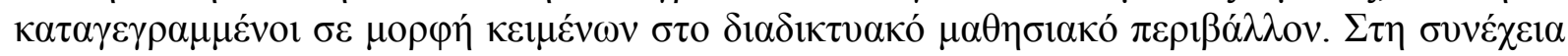

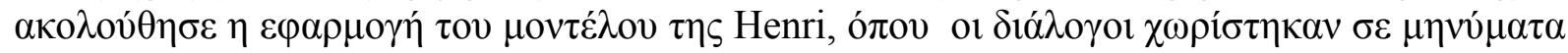

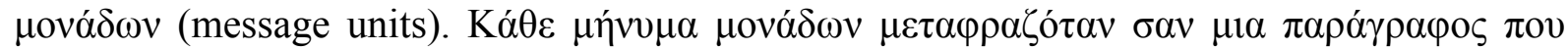

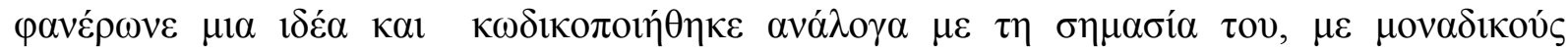

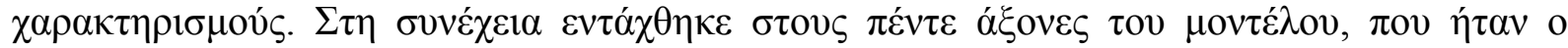

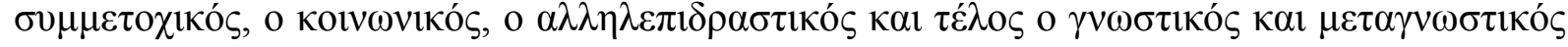
ákovas.

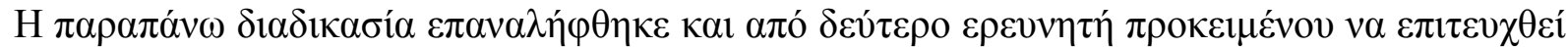

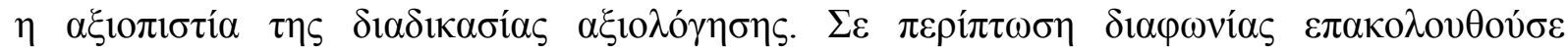

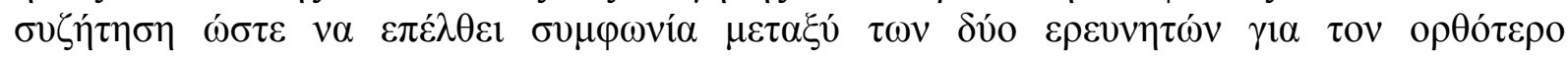

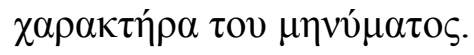

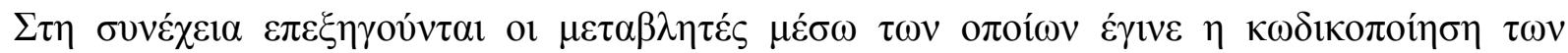

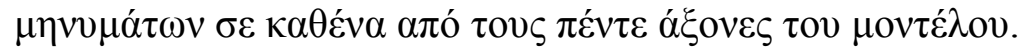

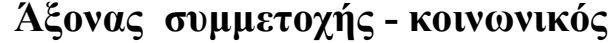

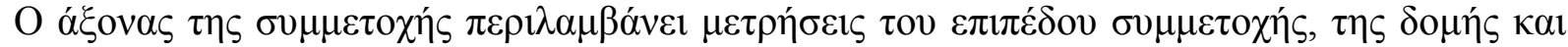

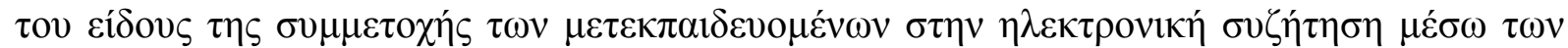

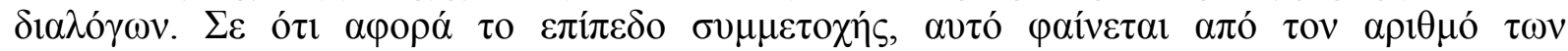

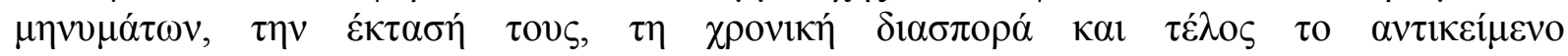
$\delta 1 \alpha \pi \rho \alpha \gamma \mu \alpha ́ \tau \varepsilon v \sigma \eta \varsigma$ ฑं $\theta \varepsilon \dot{\varepsilon} \mu \alpha$.

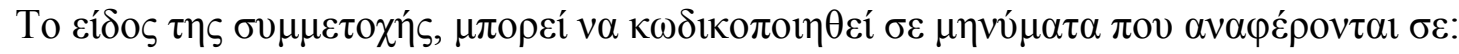

- $\Delta 1 \alpha \chi \varepsilon i \rho ı \tau \imath \kappa \alpha ́ \alpha \eta \eta v ́ \mu \alpha \tau \alpha$

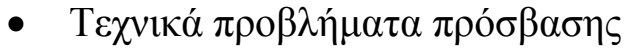

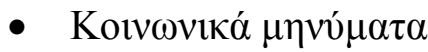

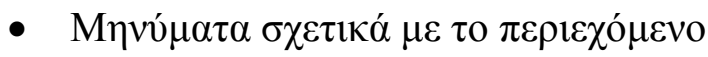

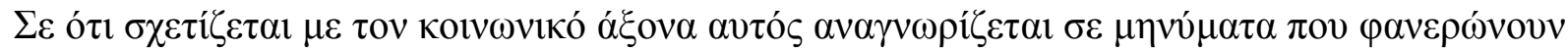

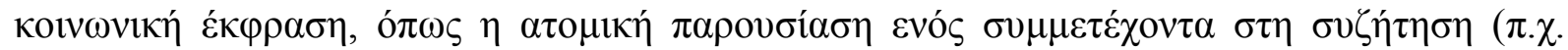

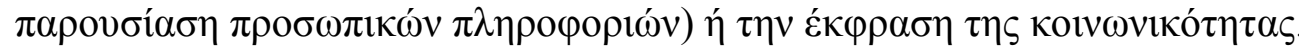

\section{Ágovas $\alpha \lambda \lambda \eta \lambda \varepsilon \pi i ́ \delta \rho \alpha \sigma \eta \varsigma$}

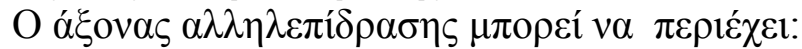

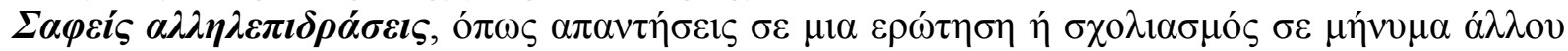
$\varepsilon \kappa \pi \alpha 1 \delta \varepsilon v o ́ \mu \varepsilon v o v$.

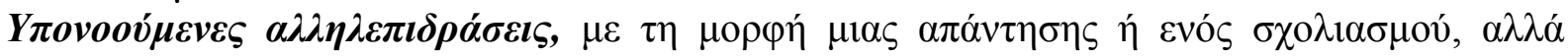

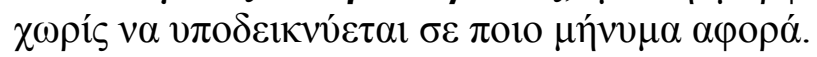

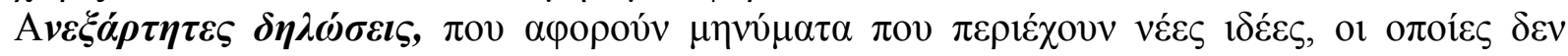

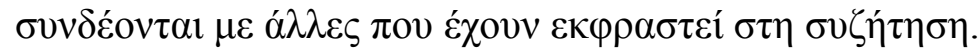

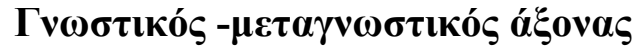




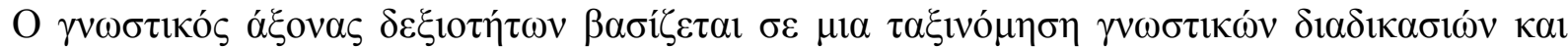

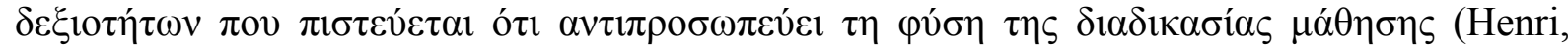

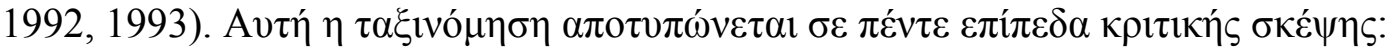

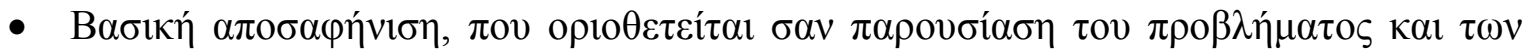
$\mu \varepsilon \rho \omega ́ v ~ \tau o v$.

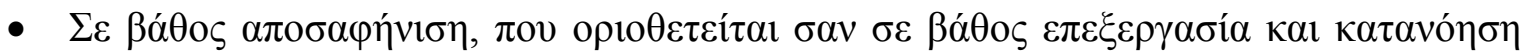

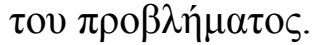

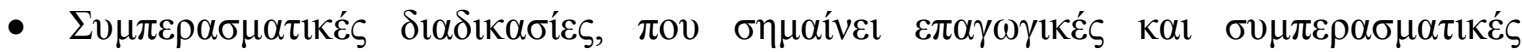

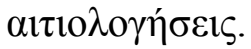

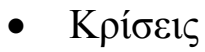

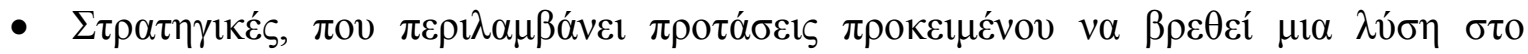
$\pi \rho o ́ \beta \lambda \eta \mu \alpha$.

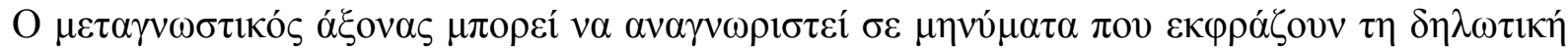

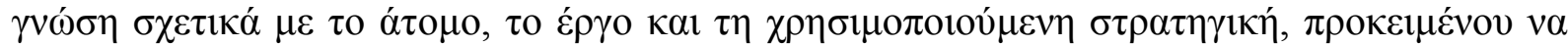

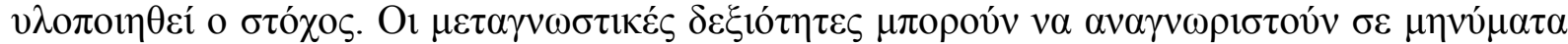

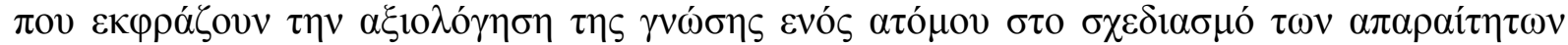

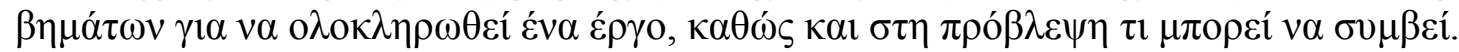

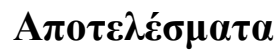

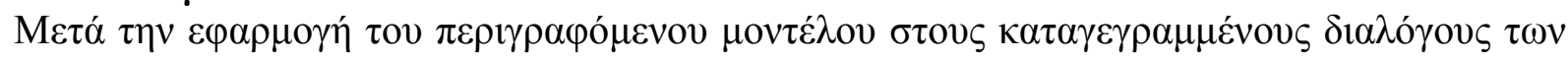

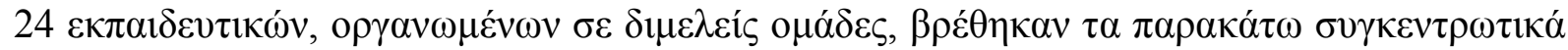

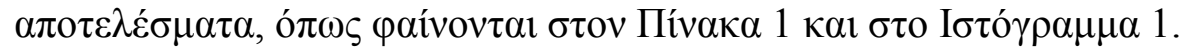

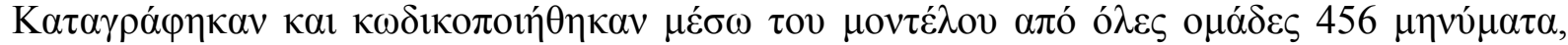

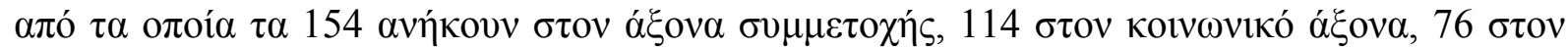

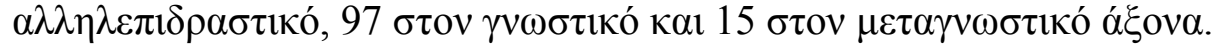

\begin{tabular}{|c|c|c|c|c|c|}
\hline$N=24$ & 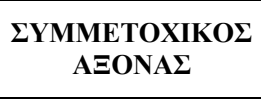 & 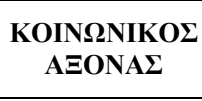 & 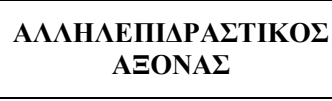 & 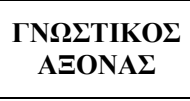 & 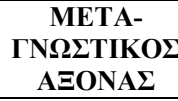 \\
\hline 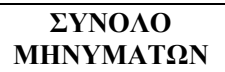 & 154 & 114 & 76 & 97 & 15 \\
\hline 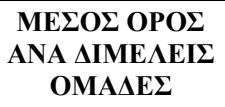 & 12,8 & 9,5 & 6,3 & 8,08 & 1,25 \\
\hline
\end{tabular}

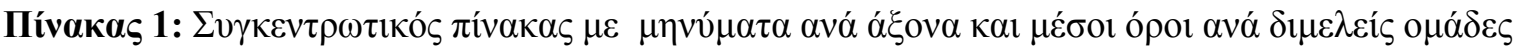




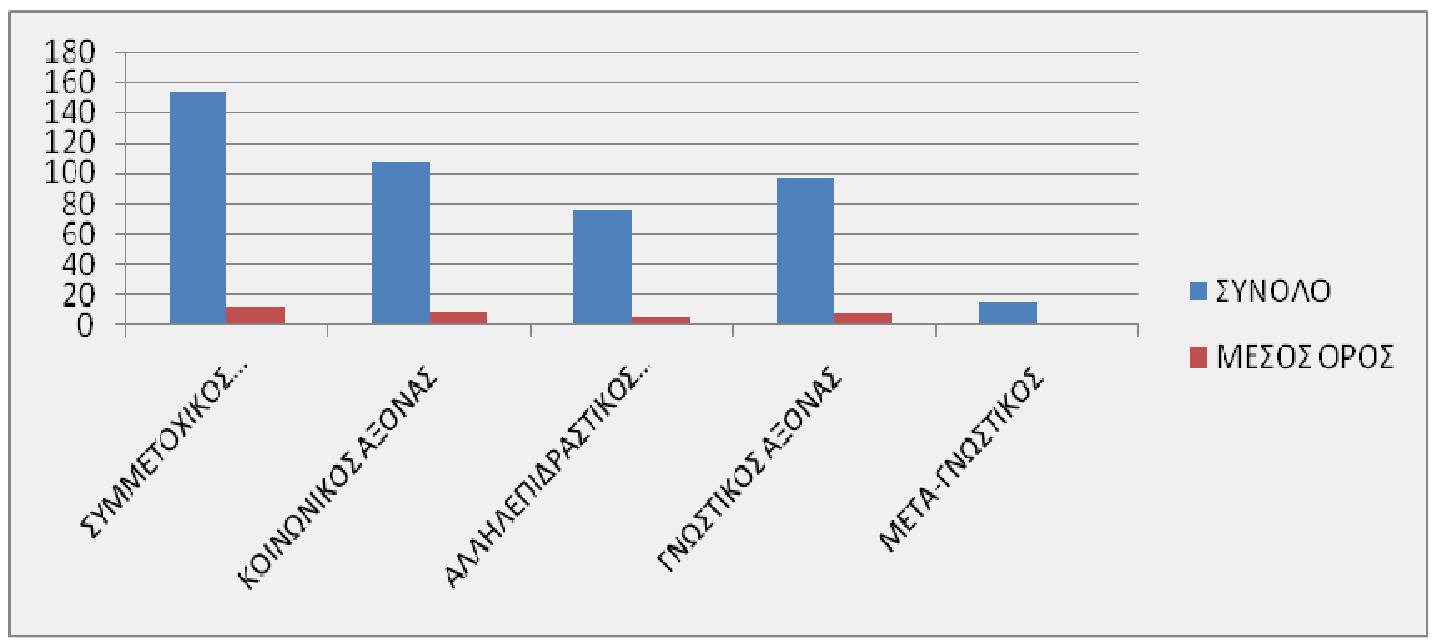

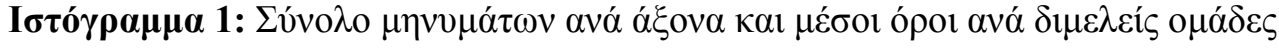

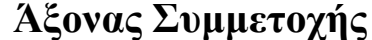

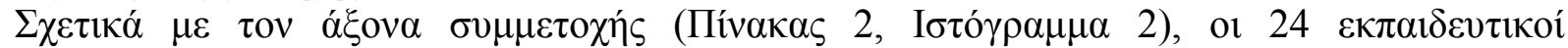

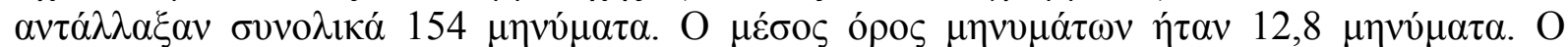

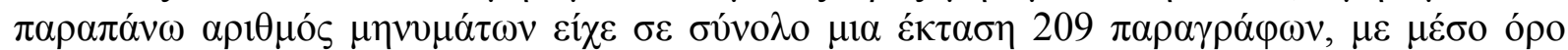

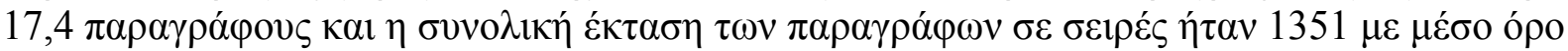

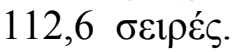

\begin{tabular}{|c|c|c|c|}
\hline & 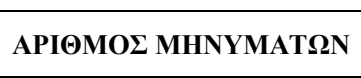 & 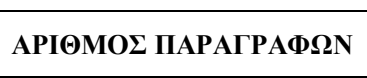 & 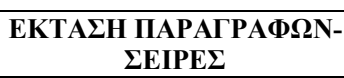 \\
\hline 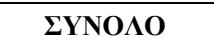 & 154 & 209 & 1351 \\
\hline 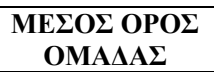 & 12,83 & 17,4 & 112,58 \\
\hline
\end{tabular}

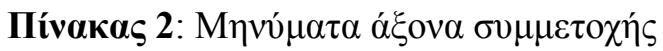

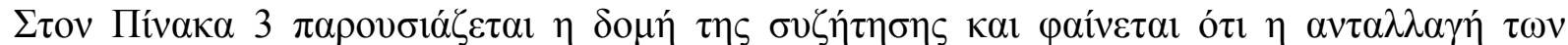

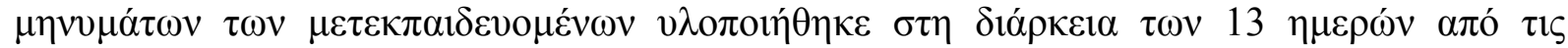

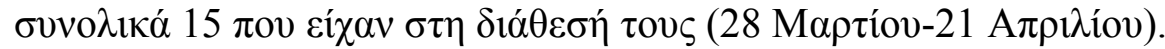

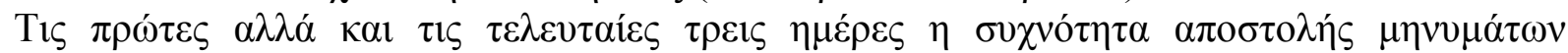

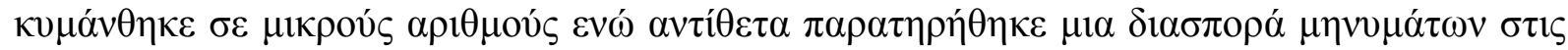

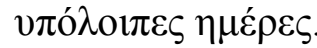

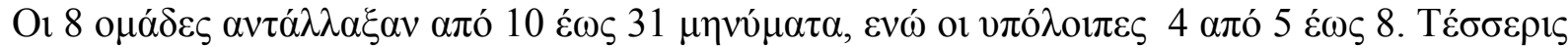

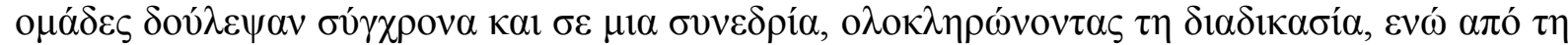

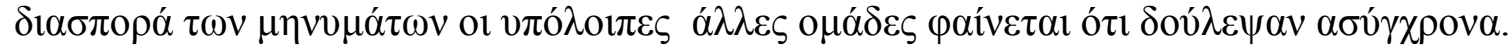




\begin{tabular}{|c|c|c|c|c|c|c|c|c|c|c|c|c|c|c|}
\hline 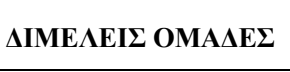 & $28 / 3$ & $4 / 4$ & $5 / 4$ & $6 / 4$ & $7 / 4$ & $8 / 4$ & $9 / 4$ & $10 / 4$ & $11 / 4$ & $12 / 4$ & $13 / 4$ & $14 / 4$ & $21 / 4$ & $\begin{array}{c}\Sigma Y N O \Lambda O \\
\text { MHNYMATSN }\end{array}$ \\
\hline$\Delta 1$ & & & & & & & & 23 & 8 & & & & & 31 \\
\hline$\Delta 2$ & & & & & & & & 10 & & & & & & 10 \\
\hline$\Delta 3$ & & & & 1 & 4 & 2 & 1 & 4 & & & & & & 12 \\
\hline$\Delta 4$ & 1 & 1 & 2 & & & & & & 2 & 1 & 1 & & & 8 \\
\hline$\Delta 5$ & & & & & & & & 11 & & & & & & 11 \\
\hline$\Delta 6$ & & 1 & & 2 & 4 & 3 & 1 & 2 & & & & & & 13 \\
\hline$\Delta 7$ & & & & & & & & & 1 & 2 & & 3 & 2 & 8 \\
\hline$\Delta 8$ & & & & 22 & & & & & & & & & & 22 \\
\hline$\Delta 9$ & & & & 1 & 1 & 1 & 2 & & & & & & & 5 \\
\hline$\Delta 10$ & & & & & & & & & & 10 & & & & 10 \\
\hline$\Delta 11$ & & & & & & & 1 & 1 & 2 & 2 & 2 & & & 8 \\
\hline$\Delta 12$ & & & & & & & & & & 5 & 11 & & & 16 \\
\hline
\end{tabular}

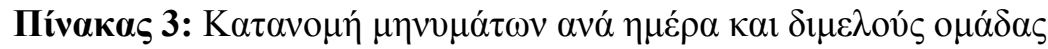

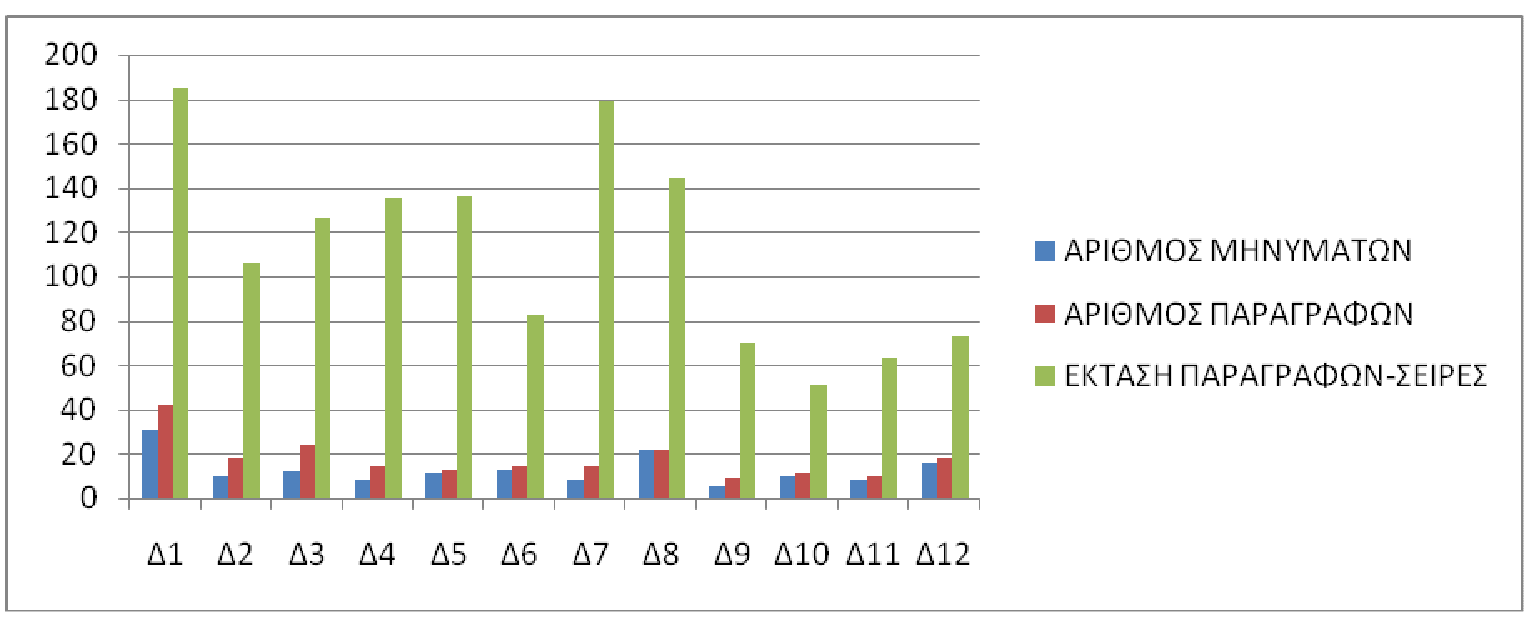

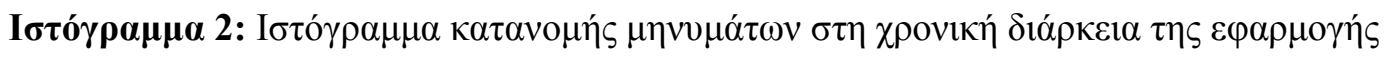

\section{Kotvฒvıкóৎ á́govas}

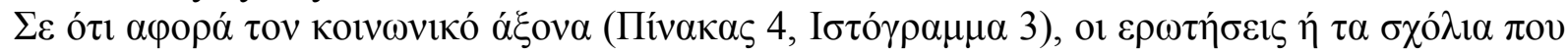

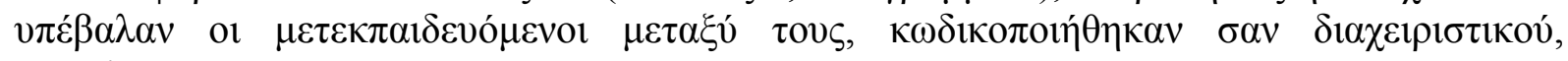

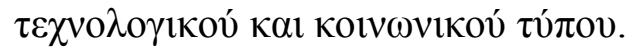

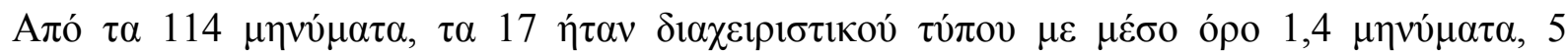

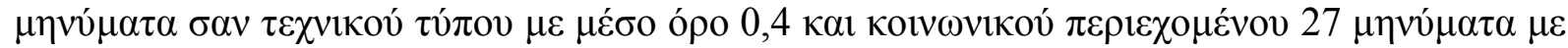

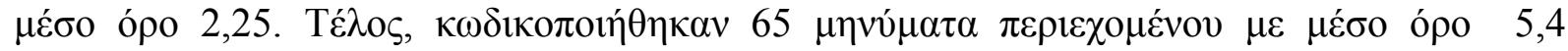
$\mu \eta v \dot{\mu} \mu \alpha \tau \alpha$.

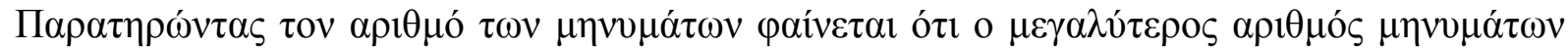

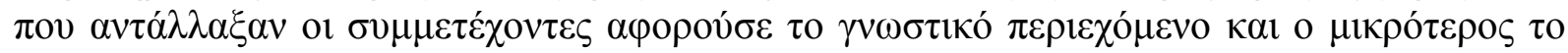

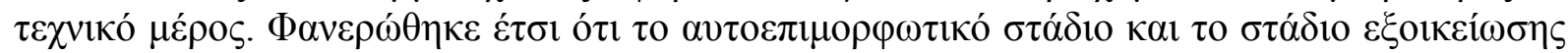

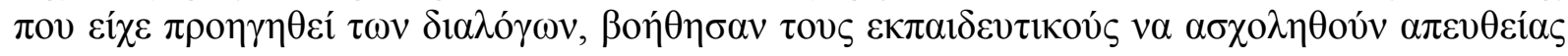

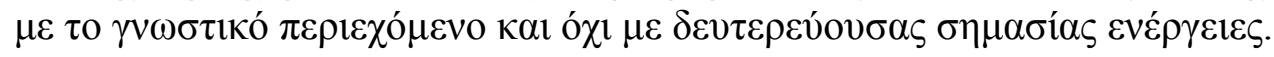




\begin{tabular}{|c|c|c|c|c|}
\hline $\mathrm{N}=24$ & 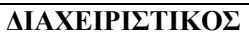 & TEXNIKO & KOIN QNIKA & ПЕPIEXOMENO \\
\hline EYNOAO & 17 & 5 & 27 & 65 \\
\hline 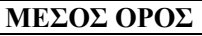 & 1,4 & 0,4 & 2,25 & 5,4 \\
\hline
\end{tabular}

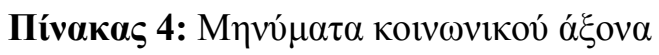

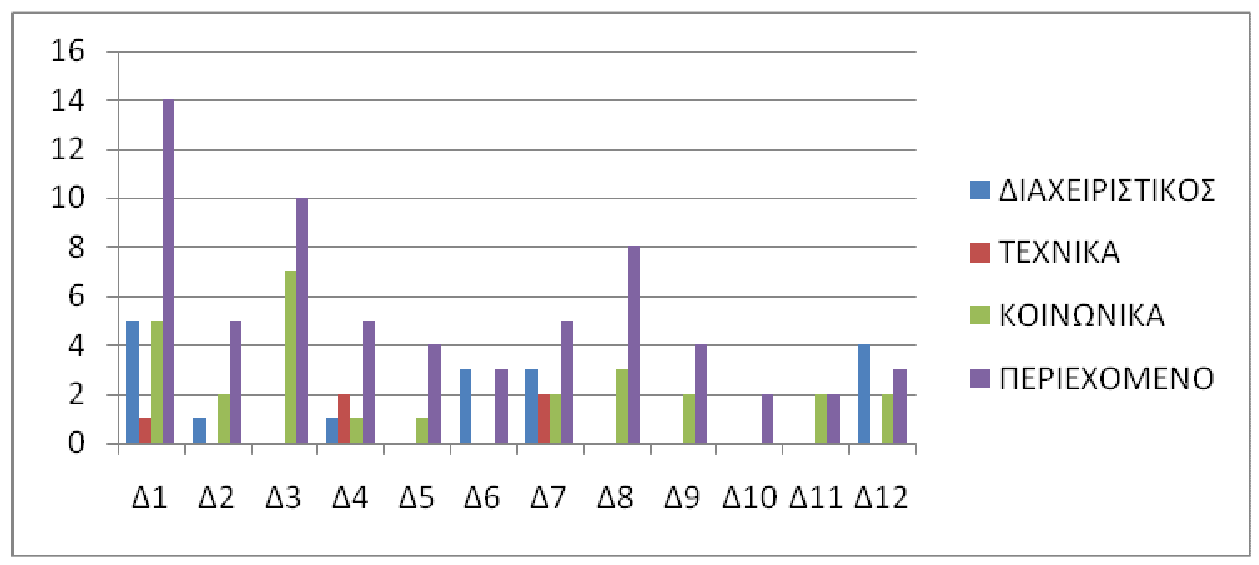

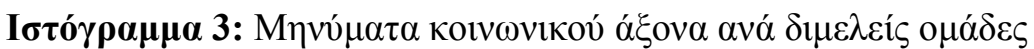

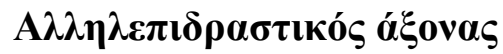

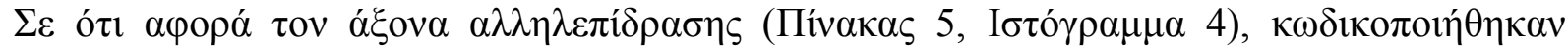

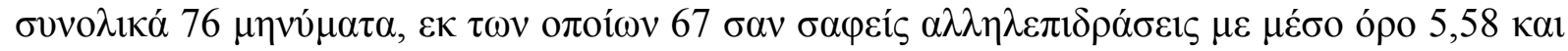

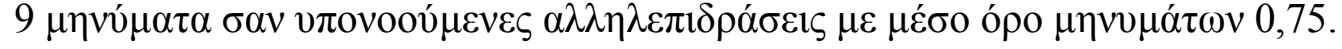

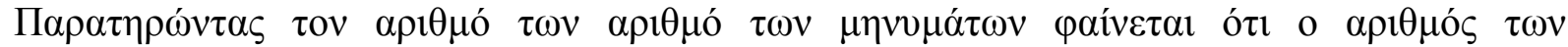

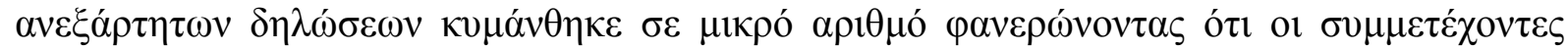

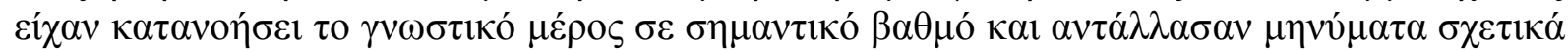

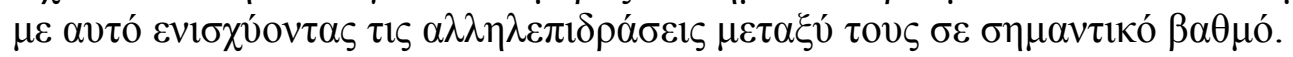

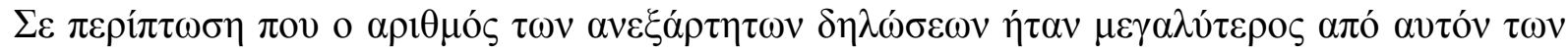

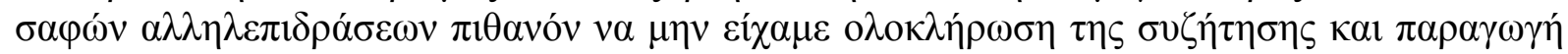

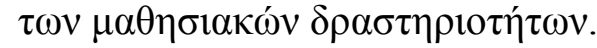

\begin{tabular}{|c|c|c|c|}
\hline$N=\mathbf{2 4}$ & 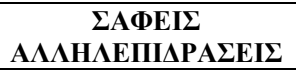 & 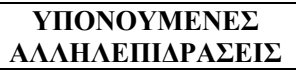 & $\begin{array}{c}\text { ANEEAPTHTE } \\
\Delta H \Lambda \Omega \Sigma E I \Sigma \\
\end{array}$ \\
\hline$\Sigma Y N O \Lambda O$ & 67 & & 9 \\
\hline ME OE OPOE & 5,58 & & 0,75 \\
\hline
\end{tabular}

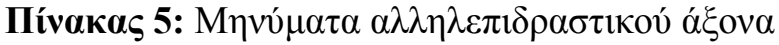




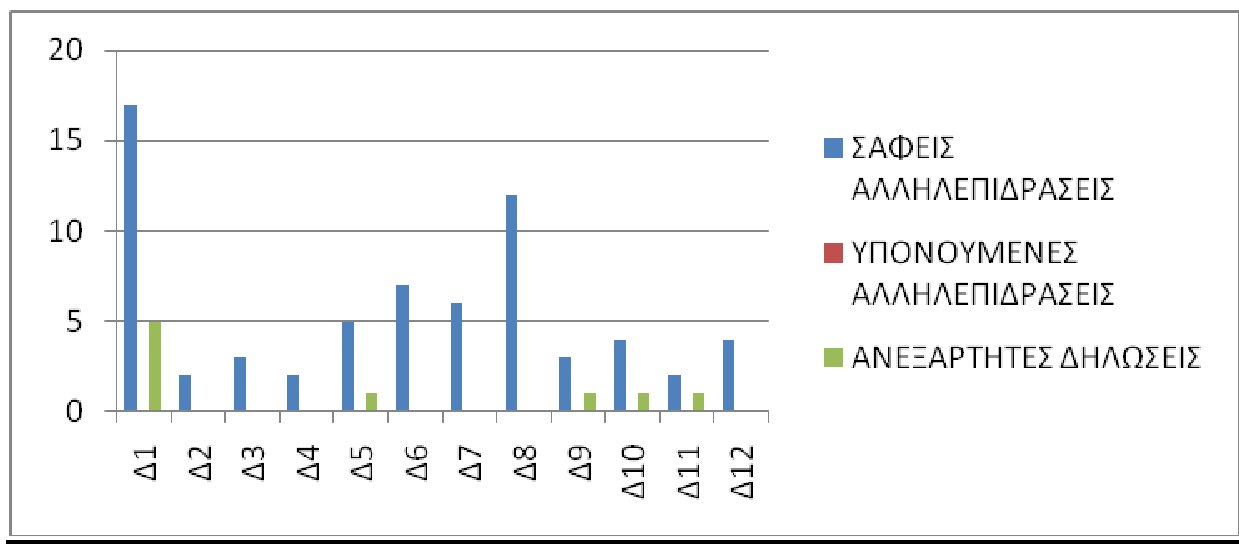

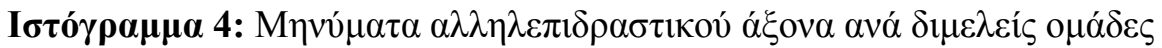

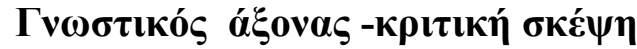

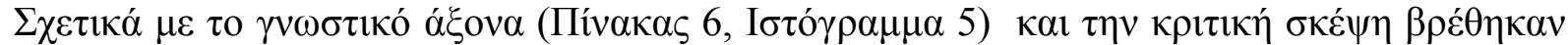

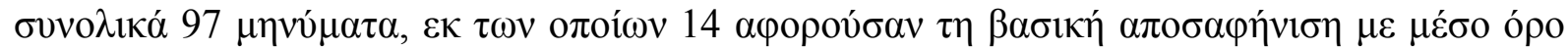

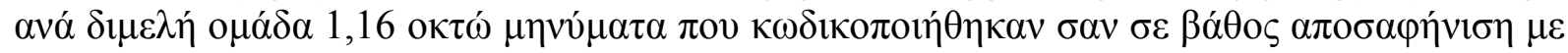

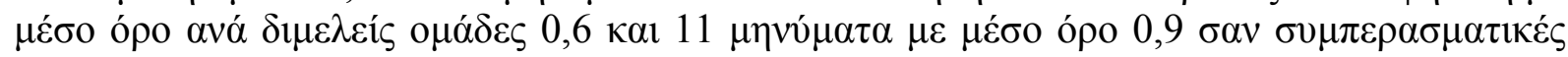

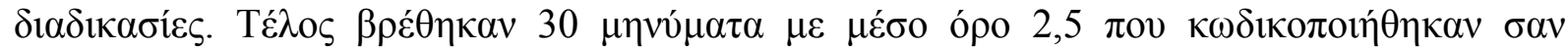

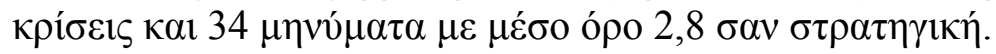

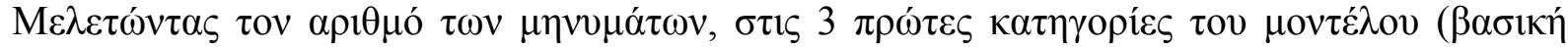

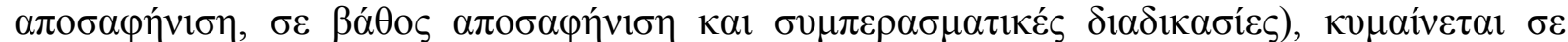

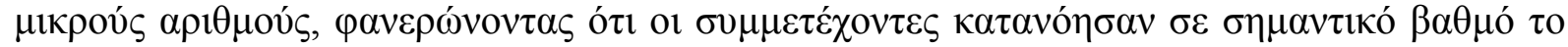

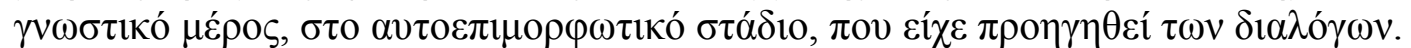

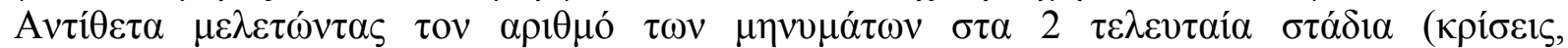

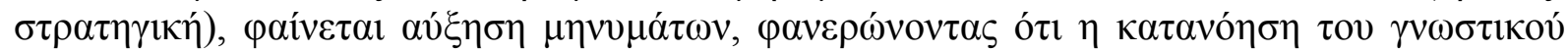

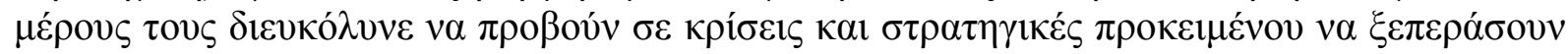

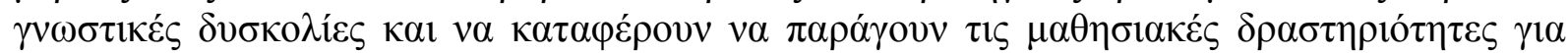

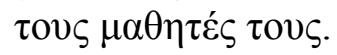

\begin{tabular}{|c|c|c|c|c|c|}
\hline $\mathrm{N}=\mathbf{2 4}$ & 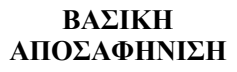 & 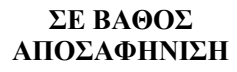 & 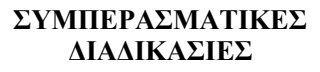 & 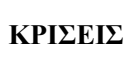 & ГТРАТНГІКН \\
\hline$\Sigma Y N O \Lambda O$ & 14 & 8 & 11 & 30 & 34 \\
\hline 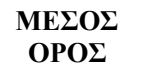 & 1,16 & 0,6 & $\mathbf{0 , 9}$ & 2,5 & 2,8 \\
\hline
\end{tabular}

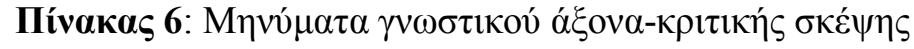




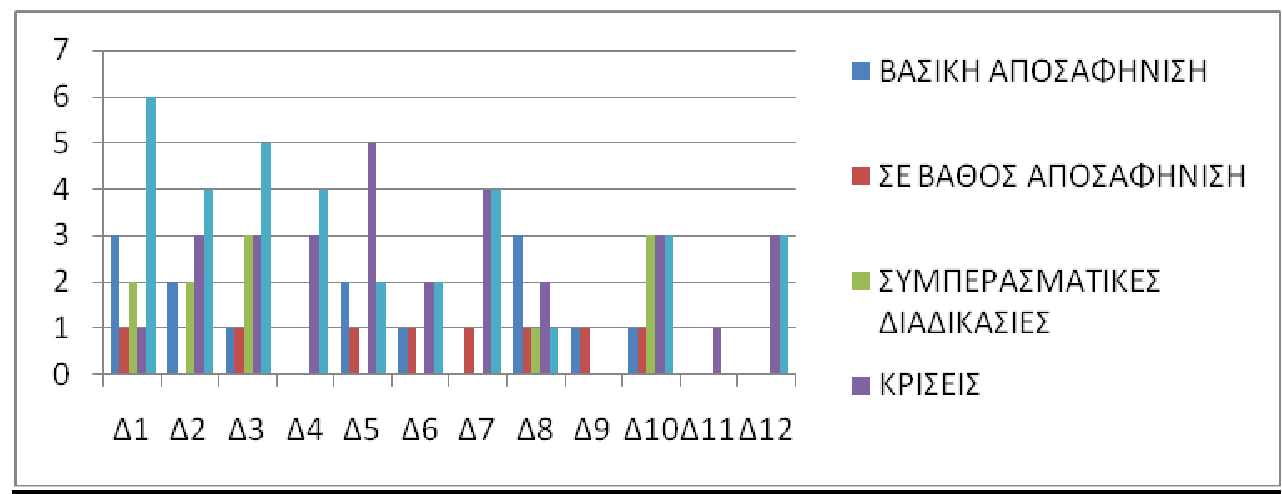

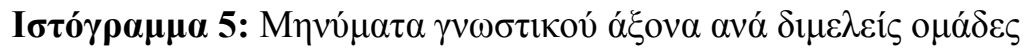

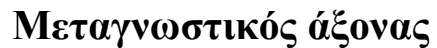

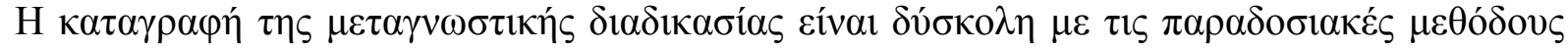

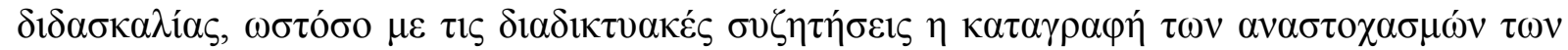

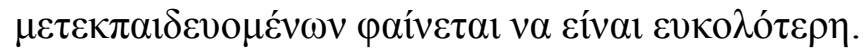

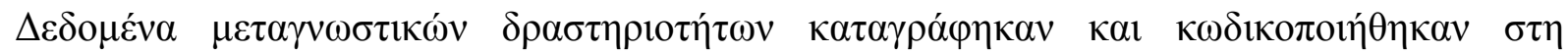

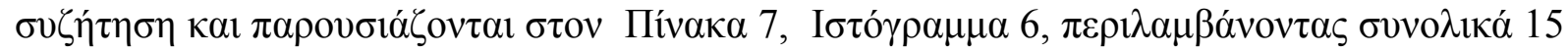

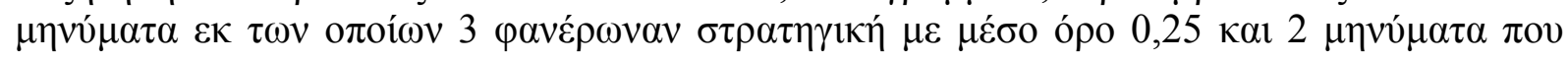

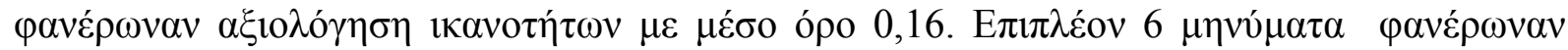

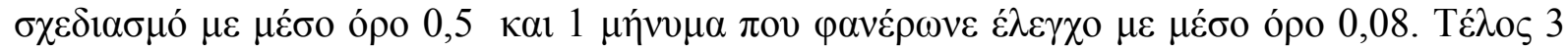

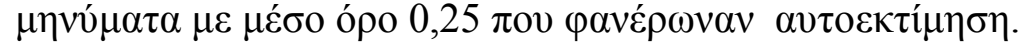

\begin{tabular}{|c|c|c|c|c|c|c|c|}
\hline $\mathrm{N}=24$ & АТОМО & ЕРГО & ЕТРАТНГІКН & 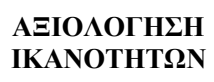 & 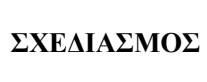 & ЕАЕГХОЕ & АYTOЕКТIMHЕН \\
\hline EYNOAO & & & 3 & 2 & 6 & 1 & 3 \\
\hline 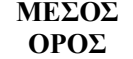 & & & 0,25 & 0,16 & 0,5 & 0,08 & 0,25 \\
\hline
\end{tabular}

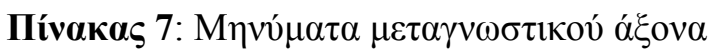

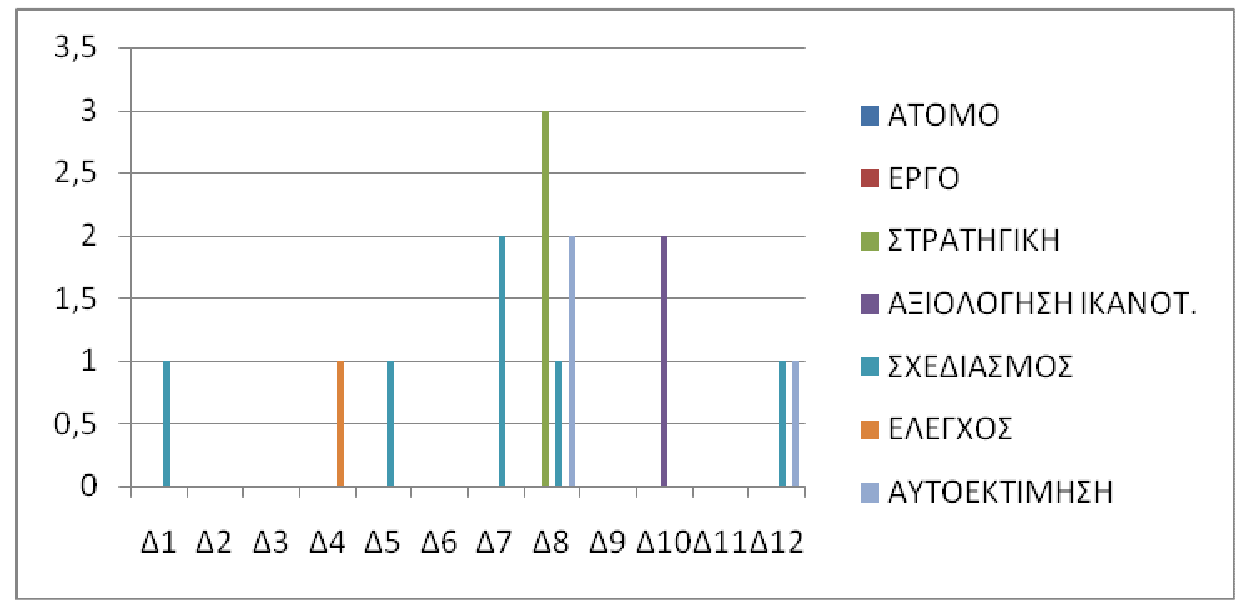

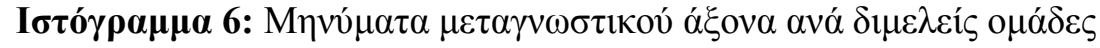




\section{$\Sigma v \mu \pi \varepsilon \rho \alpha ́ \sigma \mu \alpha \tau \alpha-\Sigma v \zeta \eta ́ \eta \eta \eta \eta$}

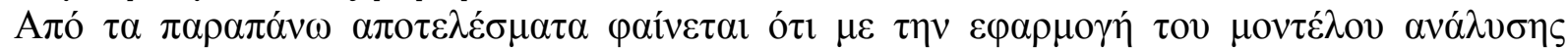

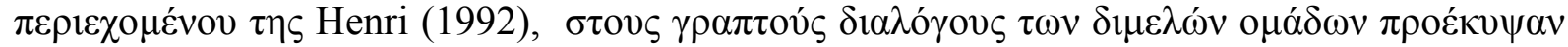

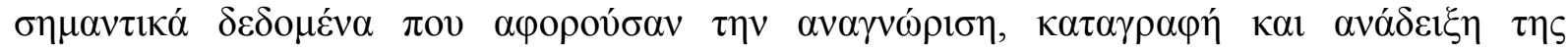

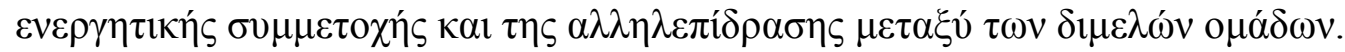

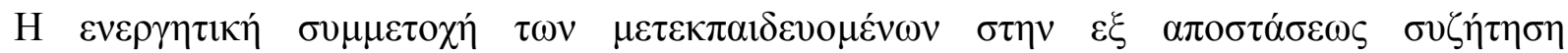

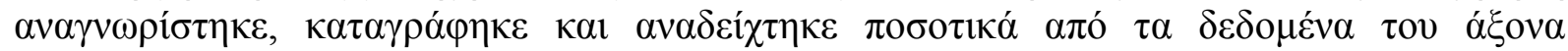

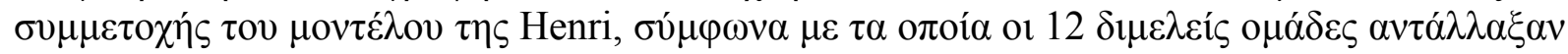

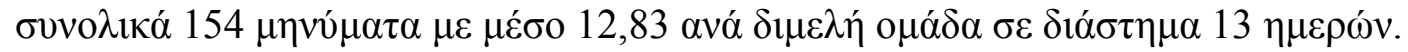

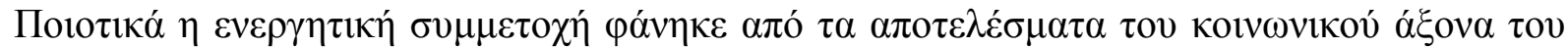

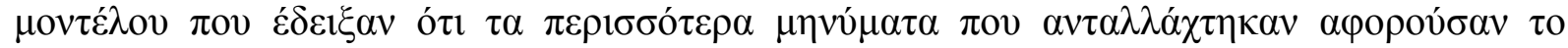

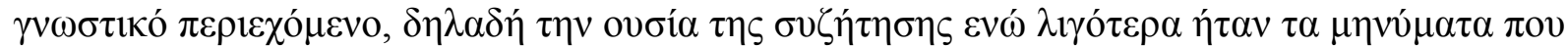

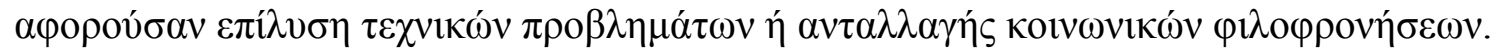

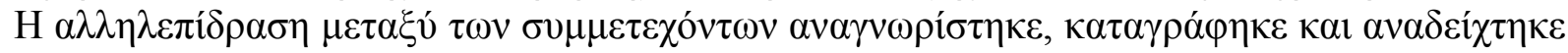

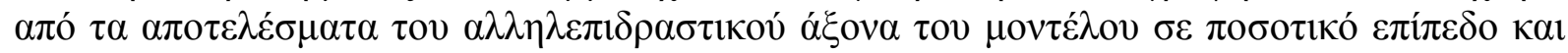

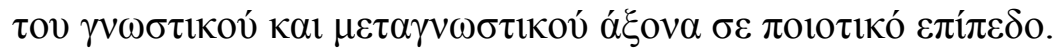

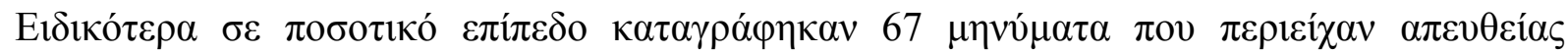

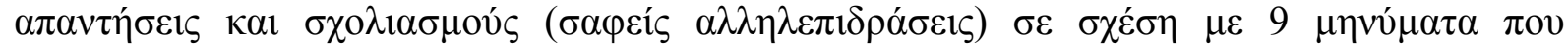

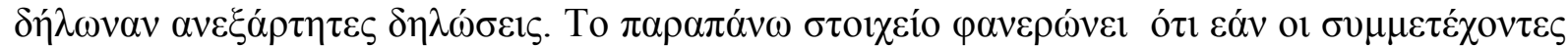

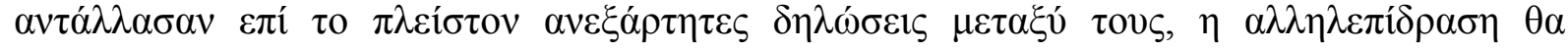

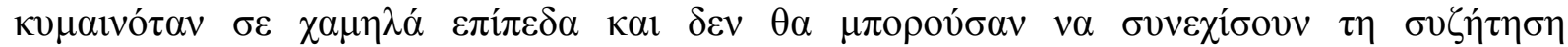

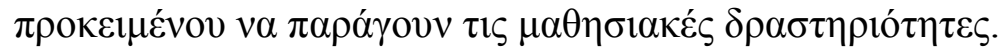

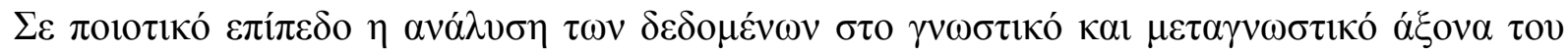

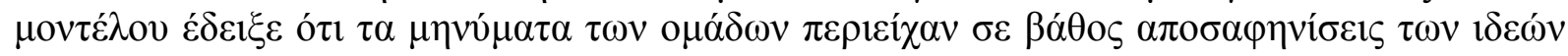

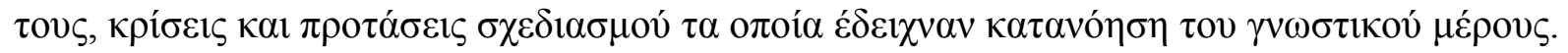

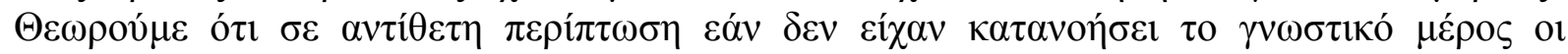

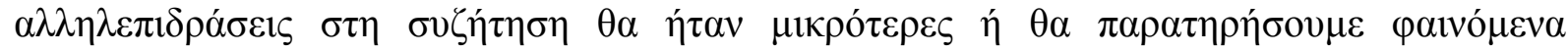

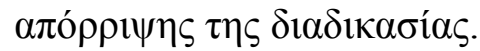

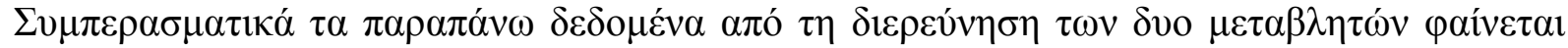

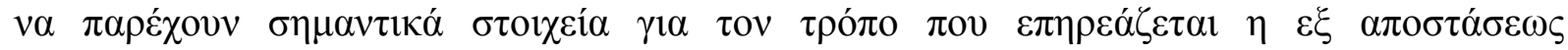

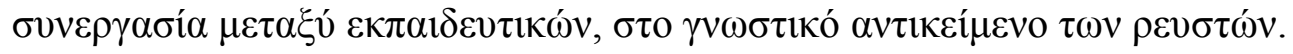




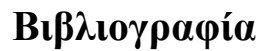

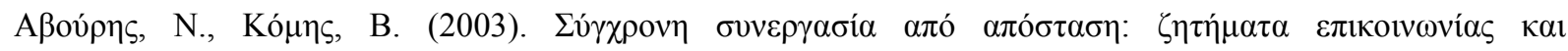

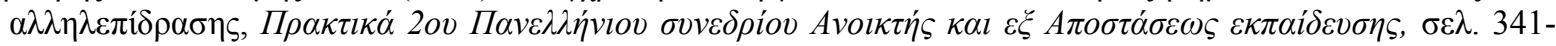
351.

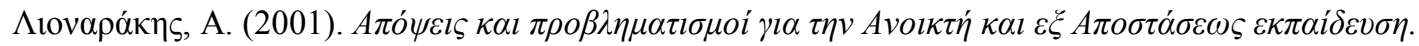

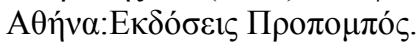

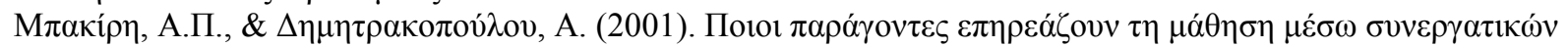

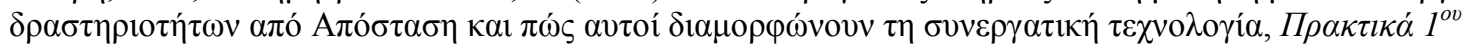

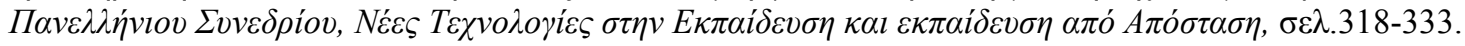

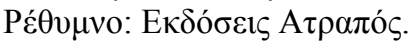

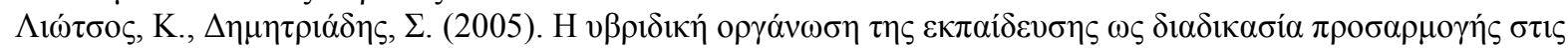

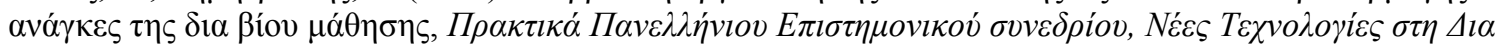

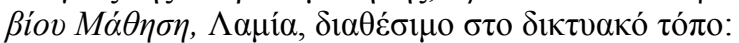
http://cosy.ted.unipi.gr/NTdiabiou2005/default.asp?id=17\&mnu=0 (11/07/2008).

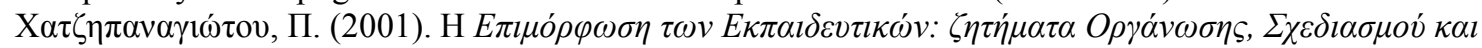

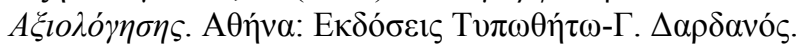

Derntl, M., \& Motsching, P., R. (2005). The role of structure, patterns and people in blended learning, Internet and Higher Education, Vol. 8, pp.111-130.

Dillenbourg, P., Baker, M., Blaye, A., O`Malley, C. (1996). The evolution of research on collaboration learning. In E. Spanda \& P. Reiman (Eds.), Learning in Humans and Machine: Towards an interdisciplinary learning science, pp. 189-211. Oxford: Elsevier

Dziuban, C., Hartman, J. \& Moskal, P. (2004). Blended Learning, Research Bulletin, Vol.7, Issue. 7.

Ginns, P., \& Ellis, R. (2007). Quality in blended learning: Exploring the relationships between on-line and face to face teaching and learning, Internet and Higher Education, Vol. 10, pp. 53-64.

Hansen, M., Spada, H. (2006). Designing instructional support for individual and collaborative demands on netbased problem-solving in dyads, Proceedings of the 7th international conference on learning sciences ICLS 06, pp.229-235. International society of the leaning sciences.

Harasism, L. (1989). Online education: A new domain. In R. Mason \& A. Kaye (Eds.), Mindweave: Communication, computers and distance education, pp.50-57. Oxford: Pergamon Press,

Henri, F. (1992). Computer conferencing and content analysis. In A.R. Kaye (Ed.), Collaborative learning through computer conferencing: The Najaden papers (pp. 115-136). New York: Springer.

Henri, F. (1993). The Virtual University: Collaborative learning through computer conferencing. Workshop: Monach University

McKenzie, W., Murphy, D. (2000). "I hope this goes somewhere": Evaluation of an online discussion group, Australian Journal of Educational Technology, Vol.16, No. 3, pp. 239-257

Paraskevas, A., Stamatis, D., Psillos, D., Molochides, T. (2003). The Design and Implementation of a Networked Virtual Classroom: A case study in the area of Fluids Physics, Journal of Information Technology Impact, Vol.3, No.3, pp.143-157 
Blended Learning: the transformation of Higher Education Curriculum

Mrs Ismini Vasileiou

University of Sheffield EdD, Lecturer in Computing at the University of Plymouth e-mail:ismini.vasileiou@plymouth.ac.uk

\begin{abstract}
This paper is a review of literature and current information related to blended learning. It will deal with several primary research issues which will include the redefining of the role of student, the role of teacher/tutor, learning and the educational establishment. The paper will analyse and discuss the selection of strategies to increase interactivity and active learning, learner characteristics, learner support and operational issues.
\end{abstract}

\title{
Introduction
}

Every educational establishment and every learner/student in order to develop and improve should be able to adapt in the environment. In order to succeed in that in a way that will give results has to pick up a dynamic and flexible procedure in learning towards knowledge and skills in order to meet society's changes.

In parallel, the industrial society is developing so rapidly into the society of information. The introduction of information but also the telecommunication technologies, the high need and constant deployment of competition in the market but also the multiplication of professional skills consist a very important role in the lifelong learning of professionals. Lifelong learning of professionals has as a target the continuous improvement of knowledge and skills.

The classic approach in education shows a number of problems for both educational establishments and learners. Such problems can be the mobility of the learners, the waste of time that could be devoted to learning, the high direct and indirect cost is caused by the expenses of travelling and mainly the inability of the learner to accommodate his personal needs because they need to be informed at all times of the advancements around their profession. For these reasons the last years there is a new approach developed that seems to give some solutions in the above problems. This approach is based on the development of new technologies and more particularly bringing into play the use of multimedia and telecommunications. With regards to what technology is offering nowadays there has been a wide growth in educational approaches and there has been an adaptation of new educational standards that are based on educating with the use of educational applications through a computer and on the distance education approach. The use of a computer in education changes considerably the way in which education was conducted. The introduction of new educational material to the learners to gain a more knowledge and comprehend knowledge when and where they can. The communication between the learner and the computer creates appropriate ground for the active participation of the learner in learning. The learner stops being a viewer only in the educational process and participates with his/her own level of knowledge improving and increasing his/her experiences and understanding.

With the use of educational applications via a computer there is the option for the learners to be educated in their own pace, in their own environment and in many situations there is the possibility of adjusting the applications on their own needs. Additionally, technologies about distance education allow to the learners to observe teaching in places where they are far away from where they live. Hence, some examples the learner can have to support them in their 
studies can be the World Wide Web, White Boarding, E-Mail, Point to Point Conferencing Audio and Video, Video Conferencing, Audio Conferencing etc.

Nevertheless, because of this inactivity in the change of the traditional model of education with the existence of the tutor in a classroom, where the learner doesn't have to go in the educational establishment, it has been caused a big delay in the spread of this style of teaching and learning and at the same time it didn't succeed in its prospective results. Therefore, it has been adopted a new model of education that combines the advantages of both ways of teaching - traditional teaching and distance learning with the use of technologies - and at the same time crosses out the weaknesses of those ways/styles. This model is named Blended Learning. It basically rounds up the best teaching styles with the best technologies in order to transfer knowledge the time that the learner needs it.

As such, in this document I will discuss and analyse how the curriculum in Higher Education has changed and how this new model of teaching and learning - blended learning - can support the new needs of Higher Education. Additionally, it will conclude on how information and blended learning in more general can be used as a tool for democratic skills.

But the question here is why to refer to democracy when discussing about the curriculum and what it the link between the two. Referring to Carr's (1998) paper is obvious that the curriculum for democracy will assist society to develop in the democratic side. The primary aim of a democratic education is to develop in pupils the habit of intelligence, the habit of confronting and resolving problems through reflective enquiry, collective deliberation and rational debate (Carr, 1998). As such, schools need to provide a democratic culture. The curriculum in any contemporary democratic society always reflects the definition of democracy which the society has accepted as legitimate and true.

\section{The needs for transforming Higher Education}

Hooker (1997) claimed that "Higher Education is on the brick of a revolution". Even in 1995 Zemsky (in Hooker, 1997) stated that higher education's core values will be at risk if a larger share of the market for undergraduate education is secured by non traditional providers. Education is not a service for a customer but an ongoing process of transformation of the participant (Harvey 2002, in D'Andrea \& Gosling, 2005). As more students enter Higher Education than ever before traditional forms of teaching are under increasing pressure to change.

There is a change between the relationship of governments and Higher Education institutions and the stakeholder interaction play an important role. All around the world governments work towards including the use of IC|T in their curriculum from primary school to Higher Education (Tondeur \& Valcke, 2007). National policies identify ICT literacy as a set of competencies needed to participate in society. The findings of the eEurope 2002 committee are that all school leavers must be digitally literate in order to be prepared for a knowledge based economy (Commission of the European Communities, 2000). National government is setting goals for national Higher Education making strategic decisions and several national documents in many countries try to introduce and include ICT as a separate school subject to teach pupils a number of technical ICT skills with the view to prepare them for further studies. Such reports are the School of Education Action plan for the Information Society (EdNA School Advisory Group, 2001), the National Educational Technology Plan (US Department of Education, 2004), the Qualification and Curriculum Authority/Department for Education and Employment (1999) and the Alberta Learning (2000). 


\section{THE CURRICULUM}

What is curriculum? As with most things in education, there is no agreed definition of 'curriculum', although it is generally agreed that 'curriculum' is not the same as 'syllabus'. A syllabus is a statement of topics to be studied in the course. A 'curriculum' equally is not just a statement of intended outcomes, products, or competencies. A competent doctor, however, is one who recognises and works within the limits of their professional competence (GMC, 2006). Curriculum is much more than either of these. Theorists concern themselves with different types of curriculum (Coles and Gale, 1985). The curriculum on paper can be the statement of purpose, aims, content, experiences, materials etc. The curriculum in action is the way in which the curriculum in paper is put into practice. The curriculum learners experience is what learners do, how they study, what they believe they should be doing etc. Finally, the hidden curriculum (Snyder, 1971) includes the behaviours, knowledge and performances that the learner infers to be important.

In 1997 Hooker stated that "Higher education is on the brink of a revolution". It is true that educational institutions are microcosms of culture and the society that supports them. If the slogan in the $19^{\text {th }}$ century was "education for those who don't know and don't have", if the slogan in the $20^{\text {th }}$ century was “even more education for those who don't know and don't have" then in the $21^{\text {st }}$ century the slogan should be "education needs to be accessible and offer more quality" (Lionarakis, 2001). Bridges (2000) has also observed the radically changing nature of higher education in the last 20 years of the twentieth century. These changes are significant, not just because they provide a changing context for the higher education curriculum, but because in the broader sense of the term, which includes all that is learned by the students, not merely that which is planned by their teachers, they change the curriculum itself. Bridges (2000) examined the boundaries that gave the definition to the university and to students' experiences. These are the identity of time, the identity of place, the identity of the scholarly community and the identity of the student community. I will first look at these and then examine why these identities have changed nowadays.

\section{The identity of time}

The idea of a tightly contained academic year of intense interaction broken by long periods of separation, or even of a day in which teaching was largely confined to a period between 9.00 and 5.00, has been broken by demands for part-time evening courses, short courses, day seminars at the weekend and summer schools as well as the need in, for example, healthrelated subjects (Bridges, 2000) and teacher training for years which match the schedules of hospitals and schools and give time for extended practical experience.

\section{The identity of place}

The rapid development of the traditional universities of distance or distributed learning systems and also of franchising, validation and accreditation, enable a student to study for a degree of University $X$ at an FE college in the region, at a higher education institution overseas or at a computer at home has challenged the identity of the educational establishment. In the professional fields in more particular the development of work placements, work-based learning, school-based teacher education and clinical attachments (Bridges, 2000) have extended the Higher Education learning environment from the university into the working environment. Widespread access to email has rendered the face-to-face 
contact between student and tutor in the university and even visits to the library a rare rather than a routine part of the experience. 'The distinction between distance education and regular instruction is beginning to disappear' (Burbules \& Callister, 1999, p. 1).

\section{The identity of the scholarly community}

It has been extremely difficult to sustain as Higher Education institutions have grown exponentially and spread, to multiple sites, relied more heavily on part-time and short-term contract staff and entered into all sorts of partnerships in teaching with practitioners in the workplace (Bridges, 2000). There has been a shift from traditional collegial models towards a more managerial or corporate styles of management. As a result, faculty and staff have faced major changes to the environment in which teaching and learning takes place (D'Andrea \& Gosling, 2005).

\section{The identity of the student community}

It has similarly been rendered more diffuse as it has become larger and topographically more dispersed and as students arrive on campus (if they come at all) at different times of the day and year, are largely non-resident, represent a wider span of ages and cultural backgrounds than ever before and combine part-time work with study.

So what we understand from the above points is that the curriculum needs to be accustomed in the wide-ranging environment. The industrial society is progressing towards the information society. There is a constant multiplication of the professional skills and there is a high need of constant training of the people and the enhancement of knowledge and skills. This is also supported Sir Francis Bacon (in Dziuban et al, 2006) who claim that "knowledge is power". So the question that arises at that point is how we, as educators, transfer the knowledge in today's demanding society. 


\section{THE CREATION OF BLENDED LEARNING}

\section{Distance Learning/E-Learning}

It is a new model of education that combines the advantages of both ways of teaching traditional teaching and teaching with the use of technologies. The theoretical basis on which instructional models is based affects not only the way in which information is communicated to the student, but also the way in which the student makes sense and constructs new knowledge from the information which is presented. Currently, there are two opposing views which impact instructional design: symbol-processing and situated cognition (Bredo, 1994) but for the purpose of this document I will not go in depth in those types of design.

Until recently, the dominant view has been the traditional, information processing approach, based on the concept of a computer performing formal operations on symbols (Seamans, 1990). The key concept is that the teacher can transmit a fixed body of information to students via an external representation. She represents an abstract idea as a concrete image and then presents the image to the learner via a medium. The learner, in turn, perceives, decodes, and stores it. Horton (1994) modifies this approach by adding two additional factors: the student's context (environment, current situation, and other sensory input) and mind (memories, associations, emotions, inference and reasoning, curiosity and interest) to the representation. The learner then develops his own image and uses it to construct new knowledge, in context, based on his own prior knowledge and abilities.

The alternative approach is based on constructivist principles, in which a learner actively constructs an internal representation of knowledge by interacting with the material to be learned. This is the basis for both situated cognition (Streibel, 1991) and problem-based learning (Savery \& Duffy, 1995). According to this viewpoint, both social and physical interaction enters into both the definition of a problem and the construction of its solution. Neither the information to be learned, nor its symbolic description, is specified outside the process of inquiry and the conclusions that emerge from that process. Prawat and Floden (1994) state that, to implement constructivism in a lesson, one must shift one's focus away from the traditional transmission model to one which is much more complex, interactive, and evolving.

Though these two theories are totally different in nature, effective designers usually start with empirical knowledge: objects, events, and practices which mirror the everyday environment of their designated learners. Then, with a firm theoretical grounding, they develop a presentation which enables learners to construct appropriate new knowledge by interacting with the instruction. To quote the AI researcher, Herbert A. Simon, "Human beings are at their best when they interact with the real world and draw lessons from the bumps and bruises they get" (Simon, 1994).

Schlosser and Anderson (1994) refer to Desmond Keegan's theory of distance education, in which the distance learning system must artificially recreate the teaching-learning interaction and re-integrate it back into the instructional process. This is the basis of their Iowa Model: to offer to the distance learner an experience as much like traditional, face-to-face instruction, via intact classrooms and live, two-way audio-visual interaction. 
Perraton (1988) defines the role of the distance teacher. When, through the most effective choice of media, she meets the distance students face-to-face, she now becomes a facilitator of learning, rather than a communicator of a fixed body of information. The learning process proceeds as knowledge building among teacher and students. This is also supported by Lionarakis (2001) who states that the teacher becomes the educational material. The teacher basically supports the didactic material. The interaction between teacher and student becomes the main condition between the didactic material and the students.

Distance education systems now involve a high degree of interactivity between teacher and student, even in rural and isolated communities separated by perhaps thousands of miles. Moreover, virtual learning communities can be formed, in which students and researchers throughout the world who are part of the same class or study group can contact one another at any time of the day or night to share observations, information, and expertise with one another (VanderVen, 1994; Wolfe, 1994).

\section{Blended Learning}

Like many learning terms blended learning has the illusion of being a concrete concept. In practice it is a flexible term that means different things to different people. Shank 2006) gives a very interesting view on what blended learning is.

"Blended learning seems to mean that there will be some e-learning and some classroom learning. It is in vogue for a simple reason. No one wants to spend that much on e-learning and people in general what to preserve what they have so they made up this nice name for not changing much and called it blended learning."

Hence, blended learning is a new model that combines the advantages of both ways of teaching - traditional teaching in the classroom and distance learning with the use of ICT. In the traditional curriculum we have tutors and the curriculum supports his work (Lionarakis, 2001). In distance learning the tutor supports the curriculum (Lionarakis, 2001).

Nevertheless, the question that arises is if blended learning is something new or old. The six major waves of technological innovation in learning (EPIC, 2003) are:

1. Writing

2. Presenting

3. Broadcast media

4. Consumer storage media

5. PC and CD-ROM

6. Internet technology

Blended learning is a custom approach that applies a mix of teaching and learning delivery options to teach, support, and sustain the skills needed for top learning performance. With blended learning, the traditional learning methods are combined with new technology to create a synergistic, dynamic learning structure that can drive learning to new heights.

How does blended learning achieve this? To answer that, we have to go back to the question of what learning is, and how it achieves performance improvement-regardless of delivery mechanism. The learning model follows 4 stages 


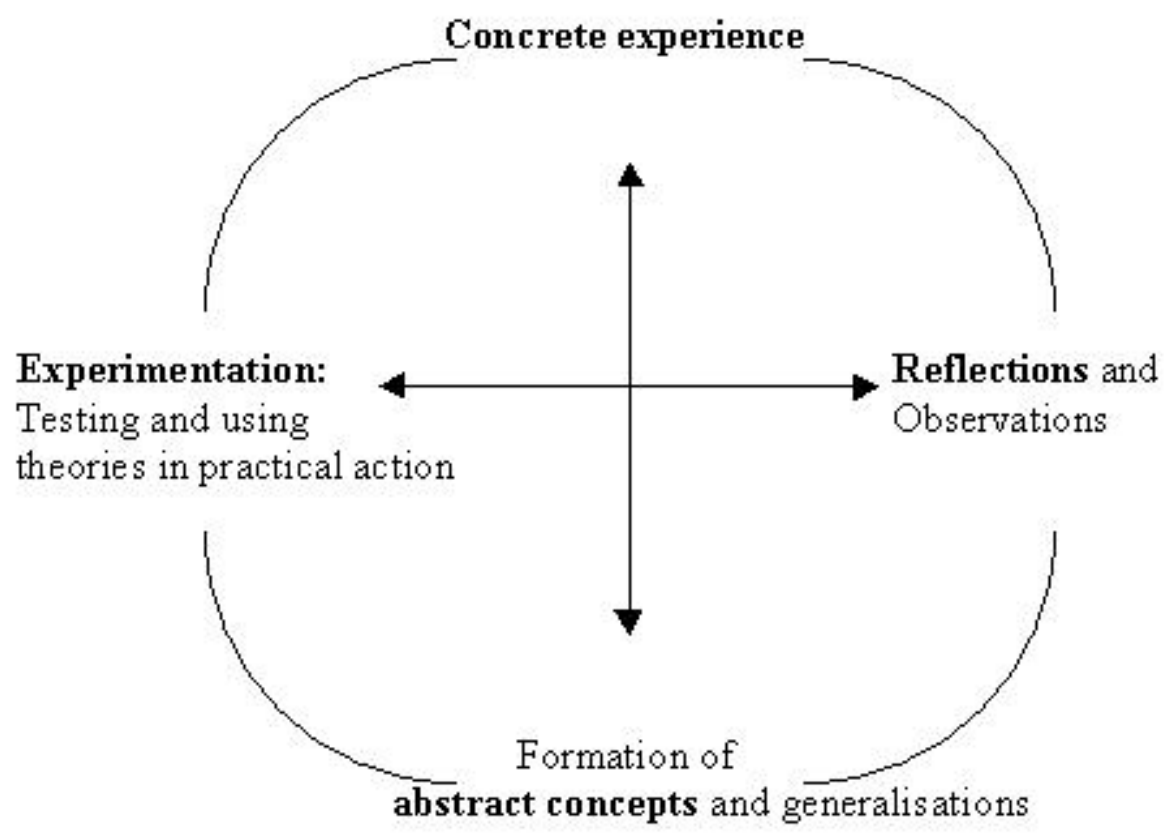

Figure 1. (Stephen Bostock, 1998)

Deep learning occurs over time. It is a process rather than an event. Only through complete processing of information, on the job practice opportunities, and feedback, will learners develop skills. Additional time is needed for the performance change to be sustained through reinforcement on the job and skill refreshers. Learner motivation and concentrated attention to learning are also key factors in the success of this learning continuum. This takes time. Therefore, it sounds as though effective learning must also be expensive. The good news, however, is that technology can compress the time spent on learning. E-learning eliminates travel time and provides individualised teaching.

The classroom provides the organisational framework and motivation, and enables people to learn through their peers' experiences. These aspects of learning can be simulated online, but classroom training will strengthen the learning experience and is the best place to deal with subtle organisational differences in practice, as well as exceptions to the rules.

Blended learning acknowledges that some stages of learning require the input and feedback of peers and the specifics of organisational approaches in order for behaviours to become embedded. Once knowledge is acquired, skills practiced, and a certain level of expertise achieved, classroom training can provide an added organisational experience to the learning process.

A blended learning approach is flexible, using the most effective delivery options for each stage of learning. It is more effective than any single form of learning at creating the results you want such as sustained behavioural change that increases the return on your training investment (EPIC, 2003). 


\section{OTHER VIEWS OF BLENDED LEARNING}

So far I have discussed how the traditional curriculum performs and what blended learning can bring into the learning in Higher Education. Consequently, this is a good place to say that there are some researchers/authors who believe that although it is widely used it can have some implications. Oliver and Trigwell (2005) argue that the term blended learning is "ill defined". They offer two arguments. In terms of philosophy blended learning relies on the idea of dichotomies which are suspect within the context of learning with the environment and becomes ineffective as a discriminating concept and it thus without purpose. The second position of the authors is that learning from the perspective of the learner is rarely the subject of blended learning. What is actually being addressed are forms of instruction, teaching and pedagogies. Both arguments have some implications as well. In the first argument there is the implication that using the term blended learning should either be abandoned or fundamentally reconceived. In the second argument, the term learning should be abandoned.

So what Oliver and Trigwell (2005) try to express through their research is that blended learning is not being used correctly. Although its popularity is expanding its clarity is not. Blended pedagogies could usefully be applied to situations where different intensities of interaction between tutors and students need to be considered. What they support is that this term lacks an analysis from the perspective of the learner. What is needed is to research and move away from manipulating the blend as seen by the teacher to an in depth analysis of the variation in the experience of the learning of the student in the blended learning context (Oliver \& Trigwell, 2005).

\section{Conclusion}

The information revolution is transforming Higher Education. Applications of digital technology are having stunning effects on the quality and quantity of pedagogical material available for the transmission of new knowledge. The traditional mode of delivery it has been that the lecturer stands in front of a group of students and talks. There two assumptions there according to Hooker (1997). The first one is that every student comes to class with the same level of background preparation. Nevertheless, no two students bring the same knowledge base to class, either in a specific field of study or in additional areas which provide a richer contextual understanding of the subject being studied. The second assumption is that all students have the same learning style and proceed at the same pace. It is true that two learning styles may be exactly the same ant that no two students learn at the same pace. Additionally, individual students will have varying levels of attention and different degrees of motivation from day to day. Hence it is clear and obvious that technology in combination with pedagogy offer us the opportunity to overcome the negative effects of both these misleading assumptions.

So why blended learning? As stated above "knowledge is power". Knowledge is a commodity and access to it is the key. The new or next generation of learners (Dziuban et al, 2006) uses sociological, cultural, economic and political perspectives rather than individual preferences. There is a fundamental difference in the way knowledge is approached today by the students. Today's students are increasingly more diverse than ever before (Dziuban et al, 2006). They are more technologically proficient and they are very often employed and more non-traditional. Therefore, students are approaching Higher Education with responsibilities above and beyond what they encounter in their classrooms. So the question that arises is if 
Higher Education can meet the needs of the present generation learner and the future one. How can Higher Education be transformed to rebuild the curriculum to meet the above needs? The solution to that is to use blended learning approaches, in other words a combination, a mixture of teaching and learning styles - a combination of web and face-to-face approaches. Some educators define blended learning approaches as "finding a harmonious balance between online access to knowledge and face-to-face human interaction" (Osguthorpe \& Graham, 2003) or the "thoughtful integration of classroom face-to-face learning experiences with online experiences" (Garrison \& Kanuka, 2004).

In conclusion, through education we transmit values and principles such as equality individual rights etc. The curriculum in Higher Education needs to transmit appropriate skills to encourage democracy. Democracy does not depend only on political conditions and processes in society. Participating persons or citizens are also important and of course dialog is necessary for democracy. Democracy in essence is a dialog between people. That means that people search for solutions to their problems by thinking together with others. The skills we need into the curriculum of Higher Education are self-critical thinking, internal dialog (systematic thinking), dialog with others and that will give us the democracy in education. In education programs we can teach the structure and processes of democracy and dialog. We can train people to participate in a meeting, to know how to make propositions and motions.

With blended learning we identify two major components for students satisfaction: learning engagement and perceived ability to communicate effectively. There is an inherent benefit in the use of educational technology for both children and teachers. This paper has critically evaluate how ICT can support the Higher Education curriculum and how it affects it. Apple (2003) noted that "ICT is part of the problem and part of the solution". Hardware alone will not enhance learning. Educators need to incorporate instructional changes, foster students' critical thinking skills and process constructivist pedagogies. Computers can engage and motivate students to learn more. Pedagogical principles are not necessarily irrelevant, but they are less sharply defined of the outset such that educators are more willing to modify their teaching strategies with different tools. Therefore there is a need to have Higher Education transformation and reform since students learn faster, better and most extensively with computers (Cuban, 2001). Referring again to Dziuban et al (2006), there is another metaphor that can be used, "knowledge is teamwork". Hence, the educator's challenge is to develop teaching and learning strategies for the blended learning environment and promote democratic skills including equal opportunities to knowledge.

The paper has tried to explore and critically analyse those areas of Higher Education that affect curriculum, how the Higher Education curriculum has been transformed because of the use of ICT and how to promote democratic skills through blended learning. By looking at the different identities involved in Higher Education - identity of time, identity of place, identity of the student community and the identity of scholarly community - I managed to distinguish what are the requirements of today's Higher Education and how Higher Education needs to meet those requirements.

Blended learning has changes significantly the Higher Education curriculum but also the needs of the society and the cultural dynamics (Apple, 2001)have affected Higher Education. So can Higher Education create a new social order? Apple (2002) is using Bernstein's notion "pedagogic device" to demonstrate the cultural configuration that enables is to uncover what exactly are the needs and when, how and why we should use blended learning techniques. 


\section{References}

Alberta Learning (2000), 'Information and communication technology: Rationale and philosophy', Alberta, Canada: Alberta Learning

Apple, M. (2002), 'Does Education have independent power? Bernstein and the question of relative autonomy', British Journal of Sociology of Education, 23(4), p. 607-616

Apple, M. (2003), 'Is the New Technology part of the solution or part of the problem in education?', in A. Darder, M. Baltodano and R.T. Torres (eds), 'The critical pedagogy reader', London: RoutledgeFalmer

Bostock, S. (1998), 'Learning Technology', $\quad$ on line, url: http://www.keele.ac.uk/depts/aa/landt/lt/docs/LearningStyles.htm, date accessed: 24/11/2007

Bredo, E. (1994), 'Reconstructing educational psychology: Situated Cognition and Deweyan Pragmatism.' Educational Psychologist, 29(1), 23-25.

Burbules, N.C. \& Callister, T.A. (1999) 'Universities in transition: the challenge of new technologies.' Paper presented to the Cambridge Philosophy of Education Conference, 18 September 1999.

Coles, C.R. and Gale Grant, Janet (1985) 'Curriculum evaluation in medical and health-care education.' Medical Education, 19(5), 405-422.

Commission of the European Communities (2000), The role of local authorities in the integration of ICT learning, Journal of Computer Assisted Learning, 18, p. 470-479

Cuban, L. (2001), Oversold and underused computers in the classroom, Cambridge: Harvard University Press

D'Andrea, V. \& Gosling, D. (2005), Improving Teaching and Learning in Higher Education, Berkshire: McGraw-Hill Education

Bridges, D. (2000), Back to the Future: the higher education curriculum in the 21 st century Cambridge Journal of Education, 30, (1)

Dziuban, C., Moskal, P., Hartman, J. (2006), Higher Education, Blended Learning and the Generations: Knowledge is power, on line, date accessed: 14/11/2007, url: http://www.blendedteaching.org/blended_and_generations

EdNA School Advisory Group (2001), Learning in an online world: The school education action plan for the information economy. Progress report 2001, on line, url: www.adna.edu.au, date accessed: 1/12/2007

EPIC (2003), 'Blended Learning', online, url: www.epic.co.uk, date accessed 15/11/2007

Garrison, D.R. \& Kanuka, H. (2004). 'Blended learning: Uncovering its transformative potential in higher education.' The Internet and Higher Education, 7, 95-105.

GMC Good Medical Practice(2006), on line, url: http://www.gmcuk.org/guidance/good_medical_practice/index.asp, date accessed 20/11/2007

Hooker, M. (1997). 'The transformation of higher education.' In Diane Oblinger and Sean C. Rush (Eds.) (1997). The Learning Revolution. Bolton, MA: Anker Publishing Company, Inc.

Horton, W. (1994). 'How we communicate. Paper presented at the meeting of the Rocky Mountain Chapter of the Society for Technical Communication.' Denver, CO, on line, url: ssi7.cs.tamu.edu/ssi/workshop\%5Cmarch06\%5Cws9\%5Cws9_2.ppt, date accessed: 24/11/2007

Lionarakis, A. (2001), 'Open and distance polymorphic education: Examination for a more qualitative approach'

Oliver, M. \& Trigwell, K. (2005) 'Can Blended Learning be redeemed?', E-Learning, 2(1), 17-26

Osguthorpe, R.T. \& Graham, C.R. (2003). 'Blended learning environments, definitions and directions.' The Quarterly Review of Distance Education, 4(3), 227-233.

Perraton, H. (1988) 'A theory for distance education.’ In D. Stewart, D. Keegan, \& B. Holmberg (Ed.), Distance education: International perspectives (pp. 34-45). New York: Routledge.

Prawat, R. and Floden, R.E. (1994) 'Philosophical perspectives on constructivist views of learning.' Educational Psychology, 29(1), 37-48.

Qualification and Curriculum Authority/Department for Education and Employment (1999), 'Information and Communication Technology: The national curriculum for England' on line, url: www.nc.uk.net, date accessed: $1 / 12 / 2007$

Savery, J.R., \& Duffy, T.M. (1995) 'Problem based learning: An instructional model and its constructivist framework.' Educational Technology, 35(5), 31-38.

Shank, R. (2006) 'Blended e-Learning', on line, date accessed 15/11/2007, http://www.celt.mmu.ac.uk/ebenchmarking/blog/?cat=2

Simon, H.A. (1994). 'Interview.' OMNI Magazine, 16(9), 71-89.

Snyder, B.R. (1971) 'The Hidden Curriculum.' Knopf, New York.

Streibel, M.J. (1991) 'Instructional plans and situated learning.' In G.J. Anglin, (ed.), Instructional technology, past, present, and future (pp. 117-132). Englewood, CO: Libraries Unlimited.

Tondeur, J. \& Valcke, M. (2007), 'Curricula and the use of ICT in education: Two worlds apart?', British Journal of Educational Technology, 38 (6), p. 962-976 
VanderVen, K. (1994, April). 'Viewpoint: The power and paradox of distance education. The On-line Chronicle of Distance Education and Communication' [On-line journal] 7(2). Online, url: http://library.georgetown.edu/newjour, date accessed 15/11/07

Wolfe, L. (1994). 'The digital co-op: Trends in the virtual community. 'Paper presented at the Writers Õ Retreat on Interactive Technology and Equipment. Vancouver, BC: The University of British Columbia Continuing Studies. 


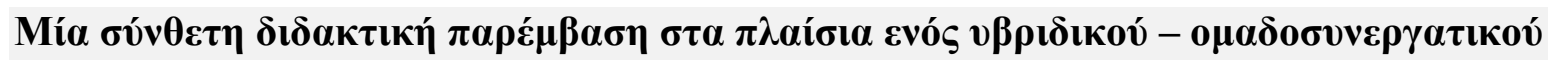

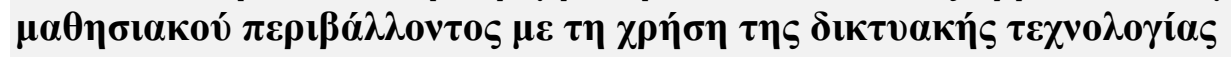

\title{
A pedagogical design of a complex instructional intervention with the creation of a hybrid collaborative learning environment
}

\author{
$\Delta \rho \mathrm{X} \alpha \mu \pi \iota \alpha o v ́ \rho\rceil \varsigma \omega ́ \sigma \tau \alpha \varsigma$

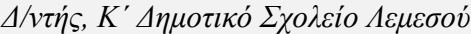 \\ e-mail:hambiaouris.c@cytanet.com.cy

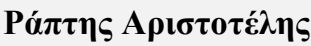

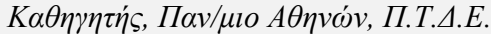 \\ e-mail:mail@raptis-telis.gr

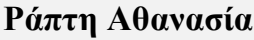

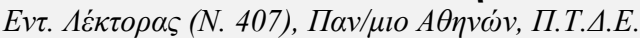 \\ e-mail: athana@raptis-telis.gr

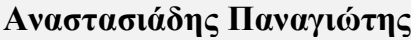

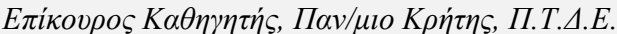 \\ e-mail:panas@edc.uoc.gr
}

\begin{abstract}
This paper refers to the pedagogical design of a complex instructional intervention with the creation of a hybrid collaborative learning environment involving distance teaching and distance collaboration among groups of students. Learning outcomes and processes have been investigated by qualitative research methods concerning: a) the psychosocial climate of the class b) the students' cognitive achievement and c) the participant teachers' critical reflection on their own educational experience. The students managed to get introduced normally to the new learning environment. This was achieved through the implementation of three phases: a) the virtual class, b) the distance collaboration and c) the distance learning communities and cooperative teaching. This innovative educational project has been based on a previous experience we gained as participants in the EU educational program called 'Odysseus' (Anastasiades, 2003). However, this particular intervention has been further developed, especially at the students' distance collaboration level through the initiation of some more open, student-centred and complex collaborative investigative learning activities. During this educational implementation the pedagogical principles of the cognitive and social constructivism have been adopted, together with some elements of the critical/transformative approach, while the method of a complex, exploratory project, with the use of ICT in a collaborative, learning environment (with or without teleconferencing) has been used as a main instructional approach.
\end{abstract}

\section{Пврі́ $\eta \psi \eta$}

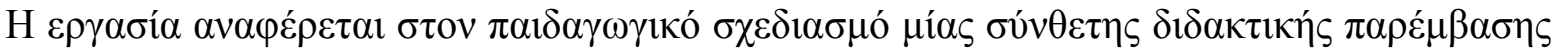

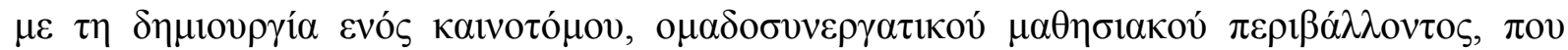

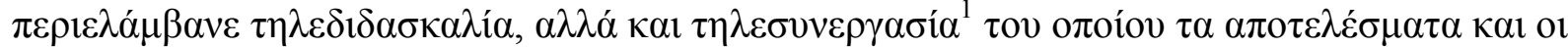




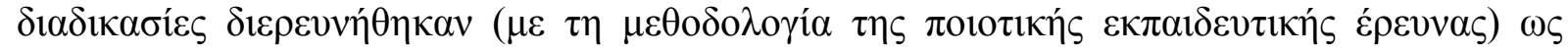

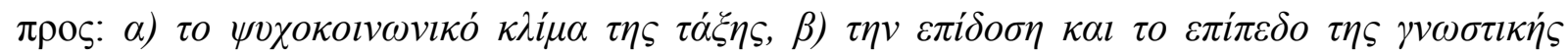

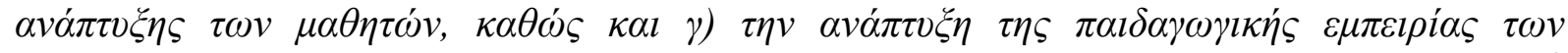

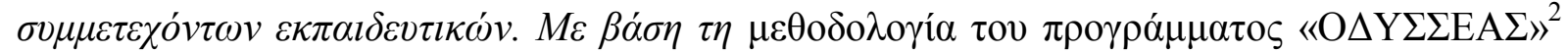

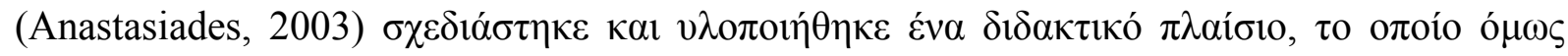

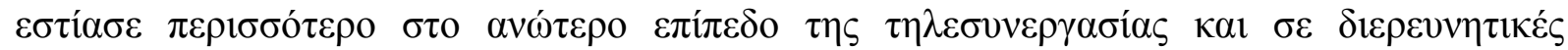

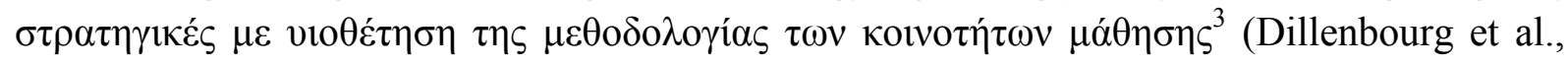

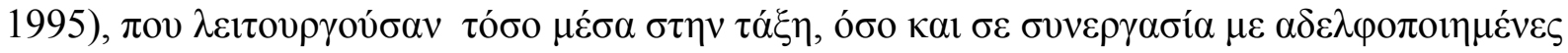

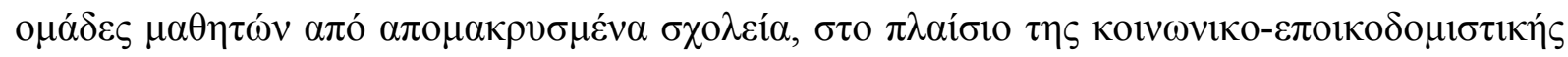

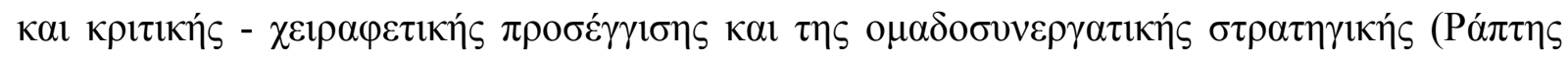

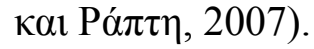

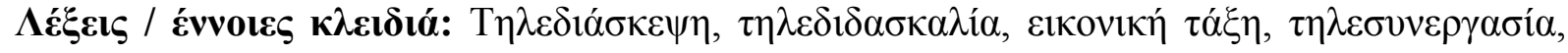

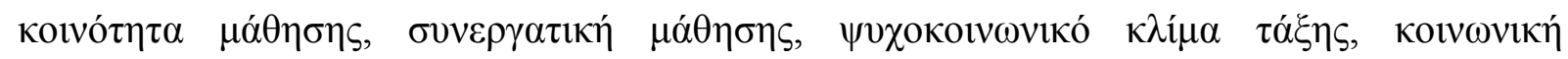
$\delta ı v \kappa o ́ \lambda v v \sigma \eta \tau \eta \varsigma \mu \alpha ́ \theta \eta \sigma \eta \varsigma$.

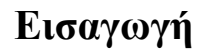

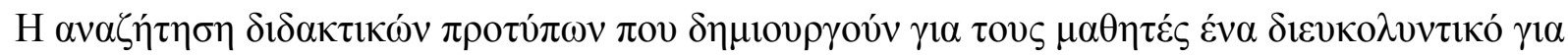

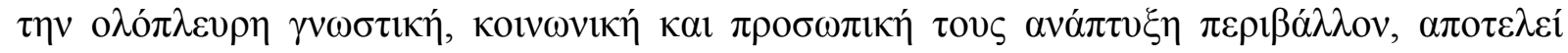

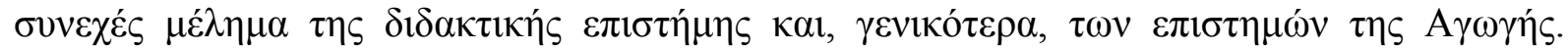

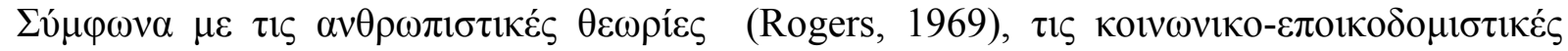

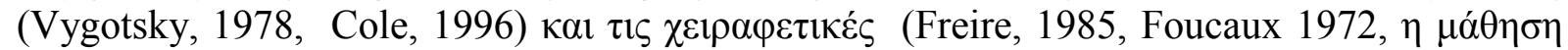

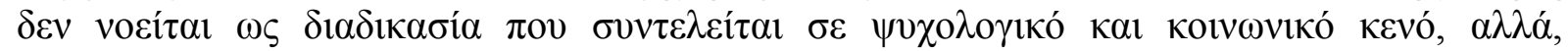

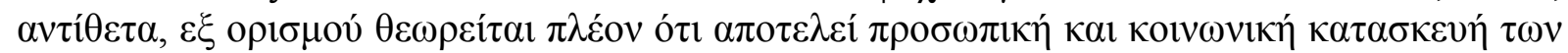

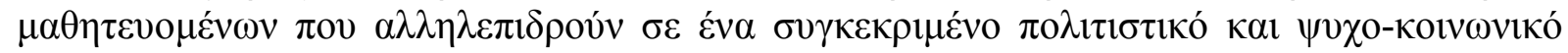

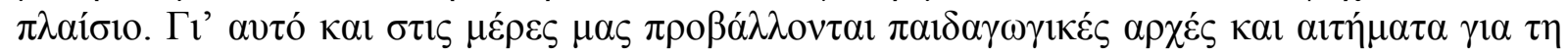

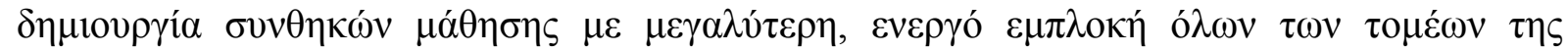

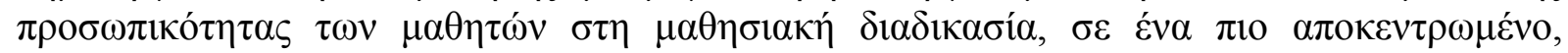

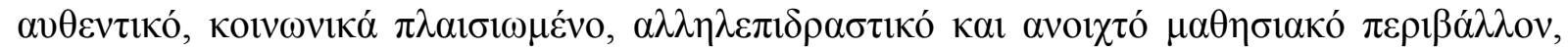

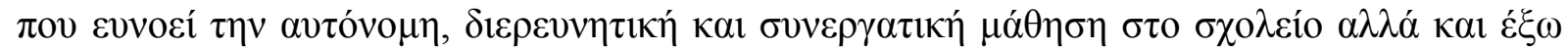

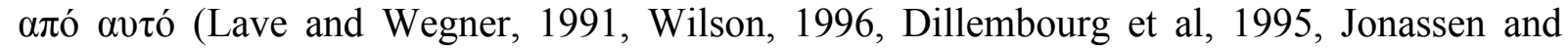
Land, 2000).

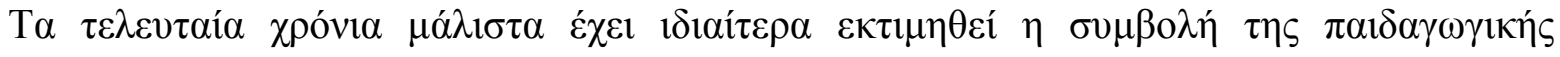

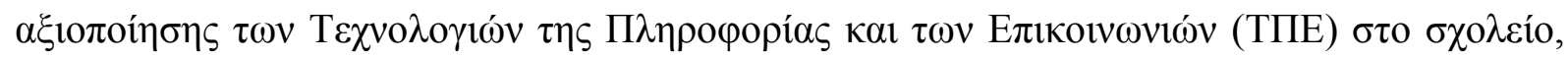

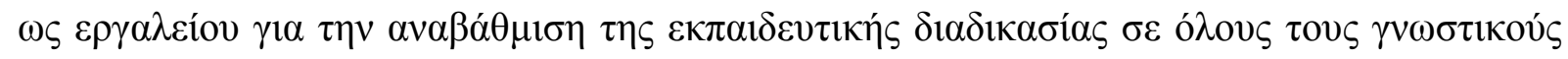

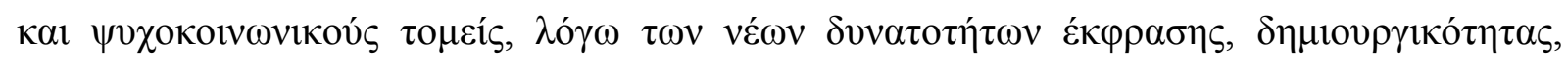

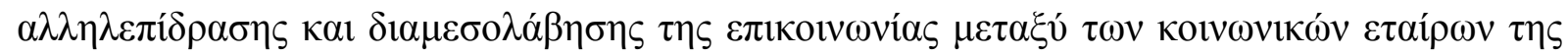

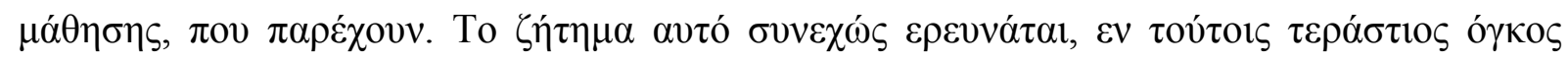

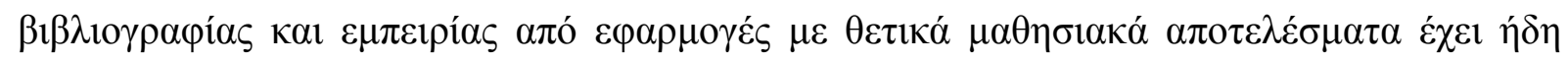

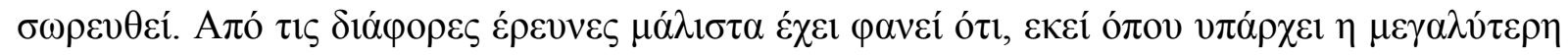

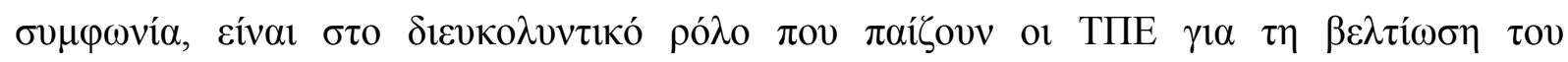

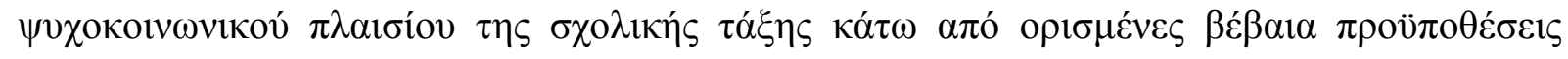

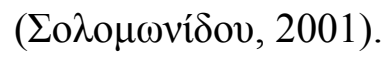




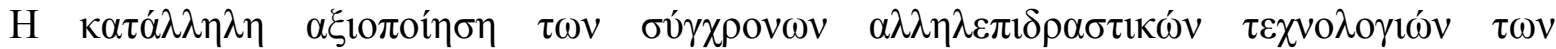

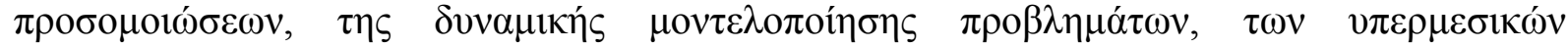

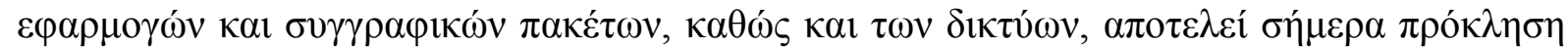

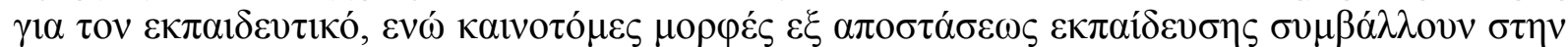

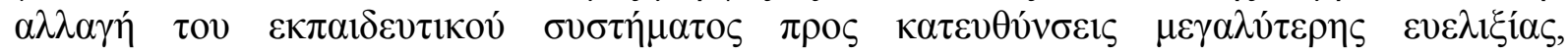

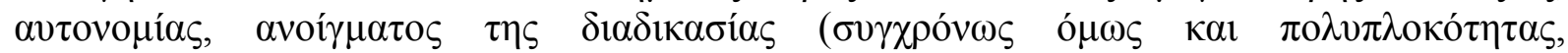

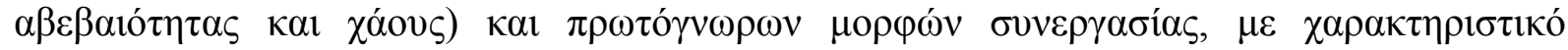

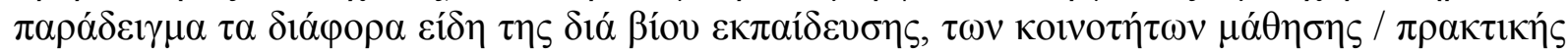
(Duffy et al, 1993, Kó $\mu \eta \varsigma, 2004$ ).

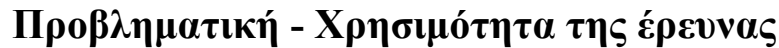

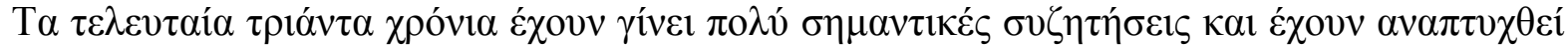

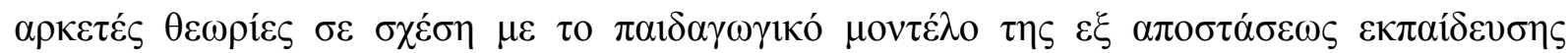

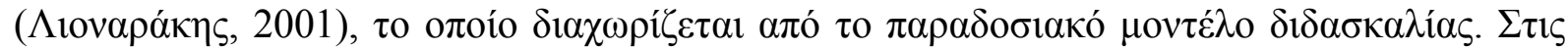

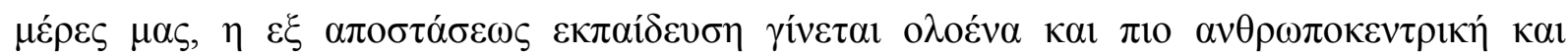

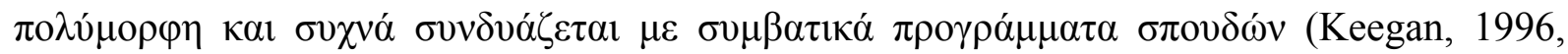

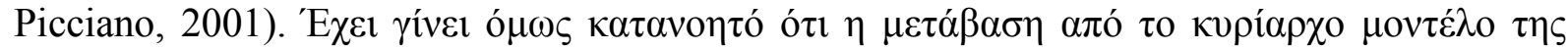

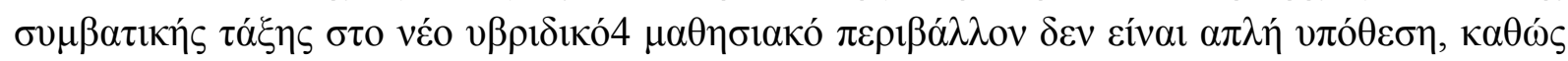

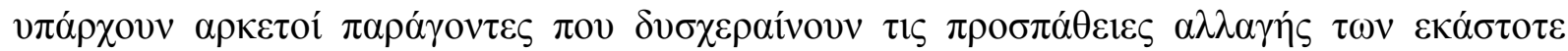

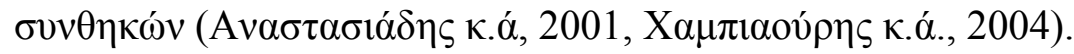

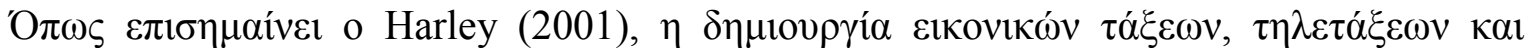

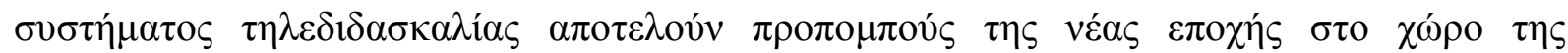

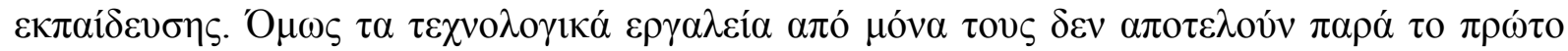

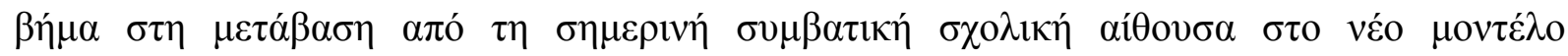

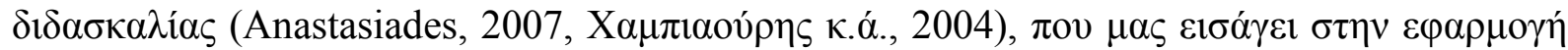

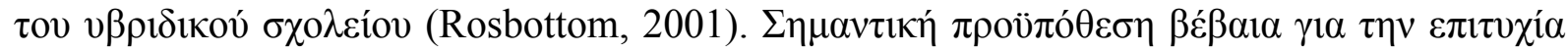

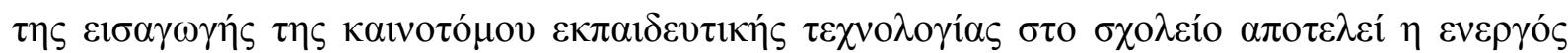

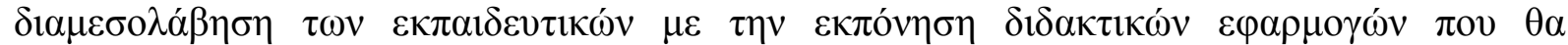

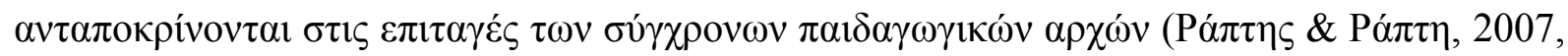

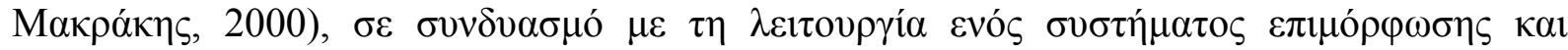

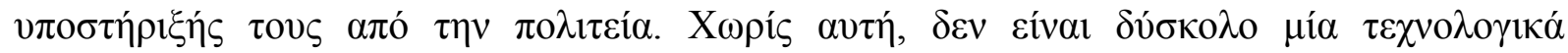

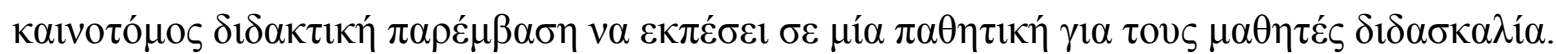

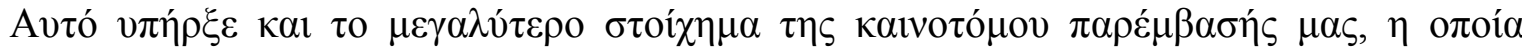

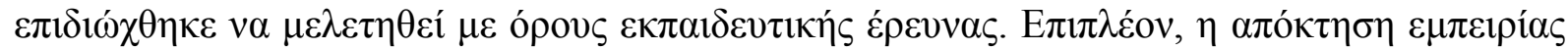

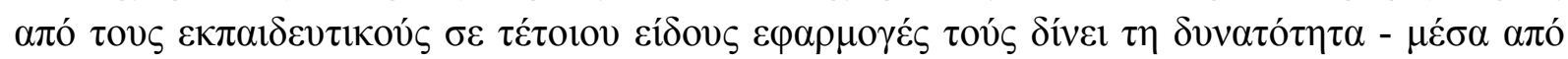

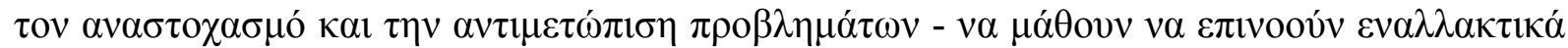

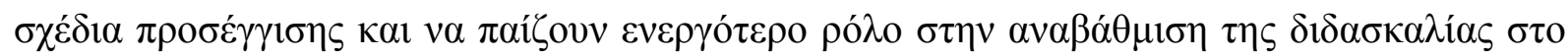
$\sigma \chi 0 \lambda \varepsilon i ́ o$.

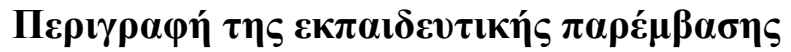

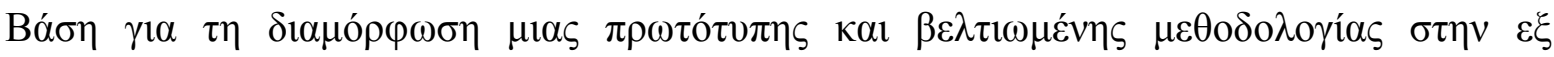

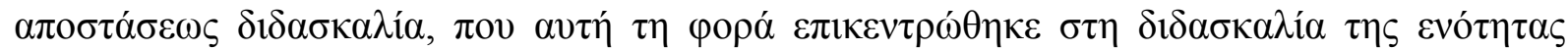

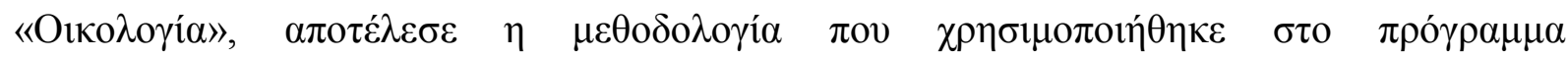

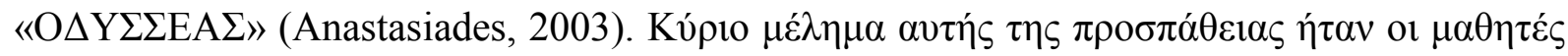




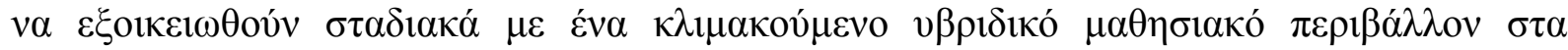

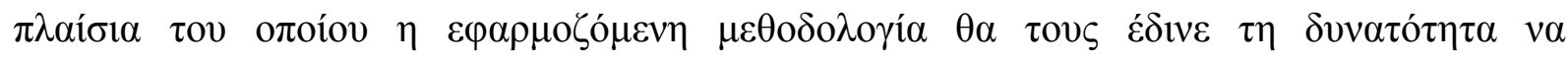

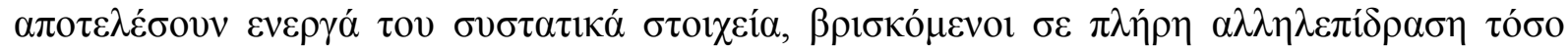

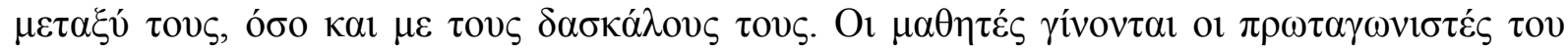

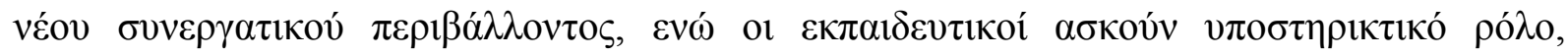

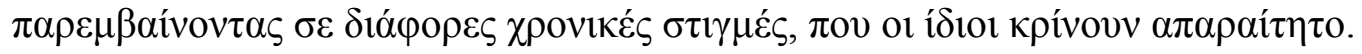

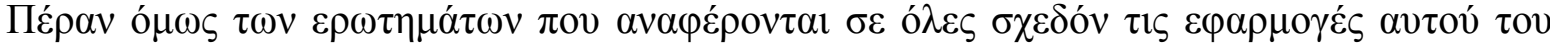

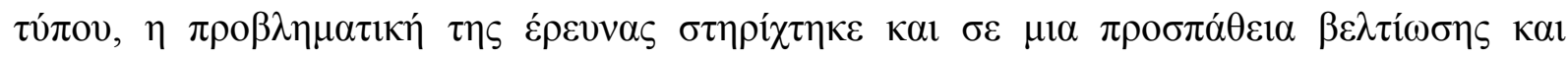

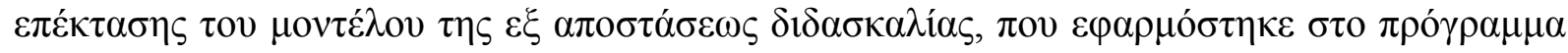

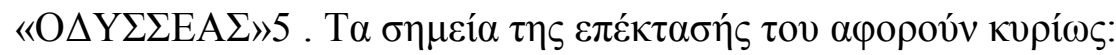

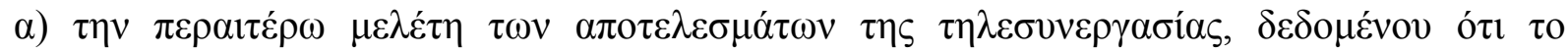

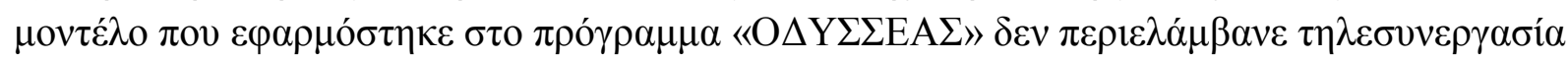

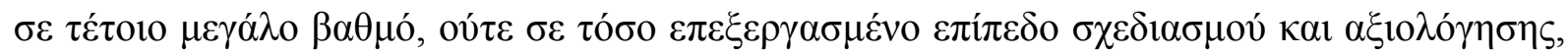

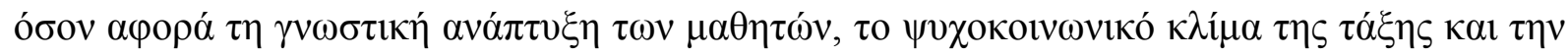

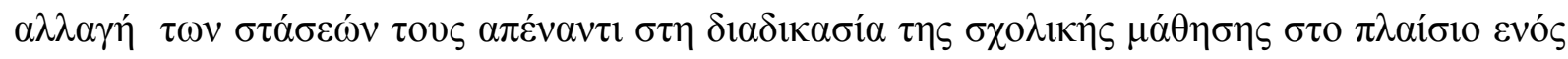

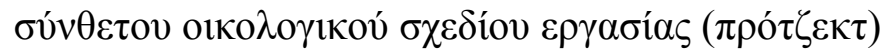

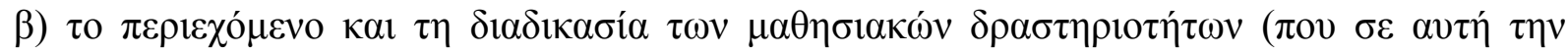

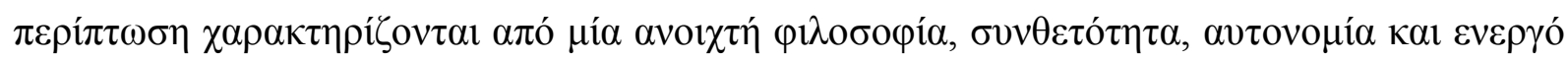

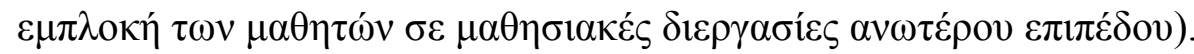

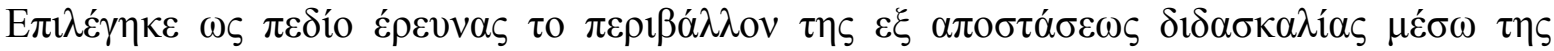

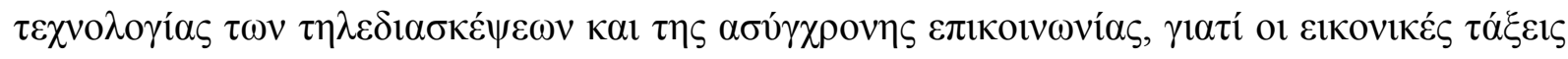

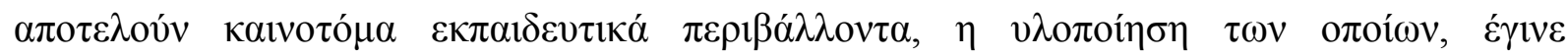

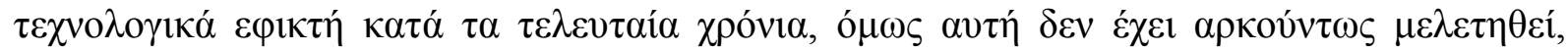

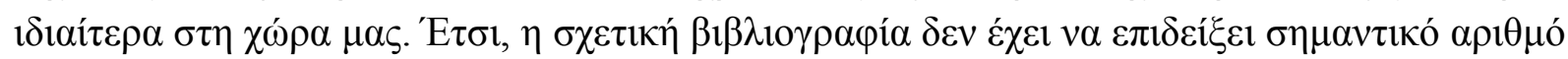

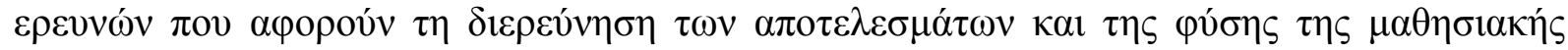

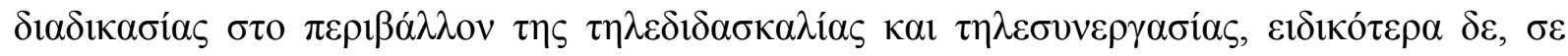

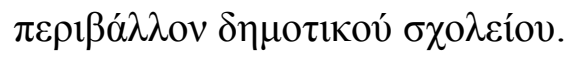

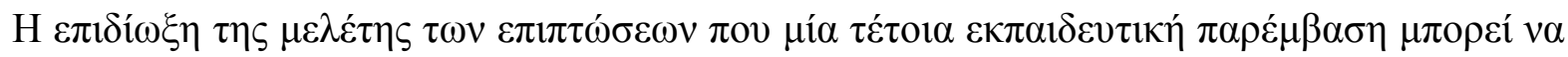

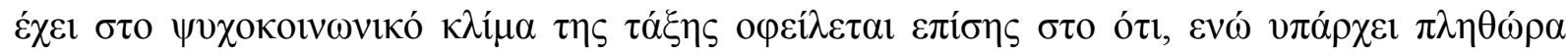

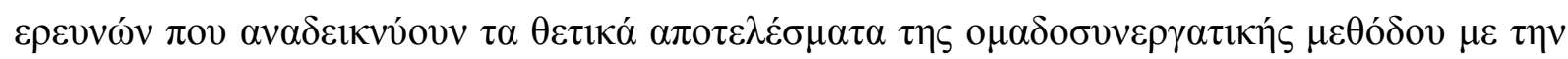

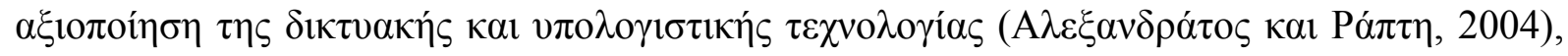

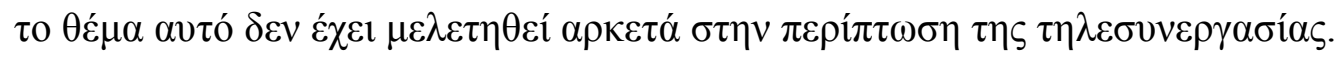

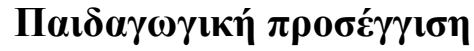

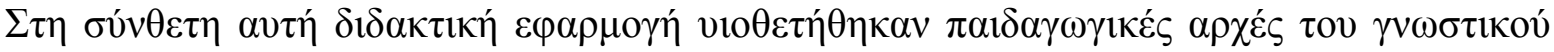

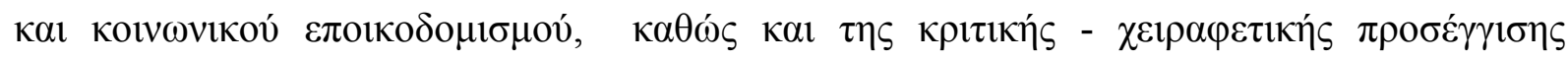

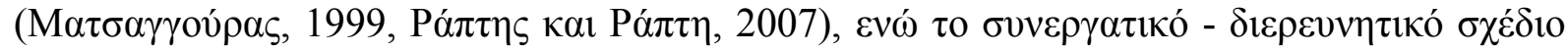

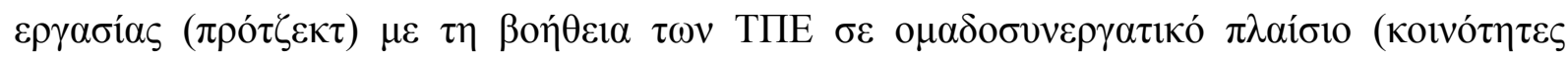

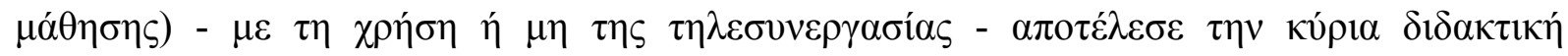
$\sigma \tau \rho \alpha \tau \eta \gamma \iota \kappa \dot{. ~}$ 


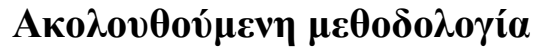

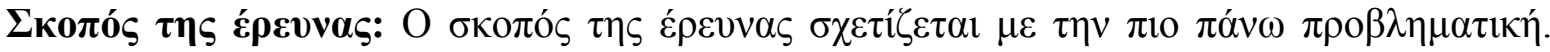

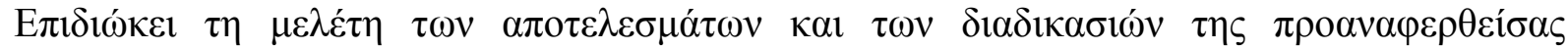

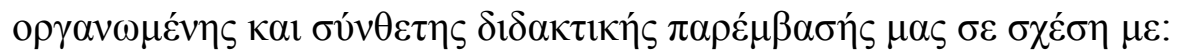

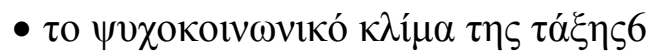

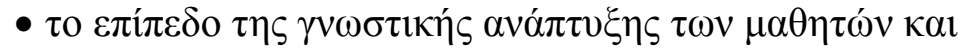

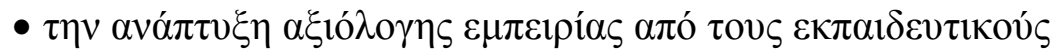

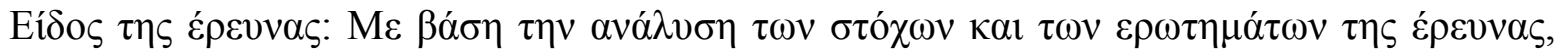

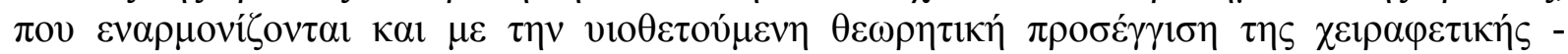

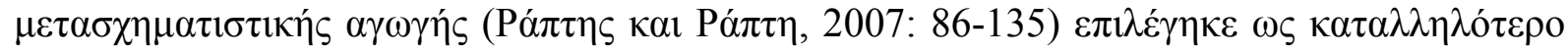

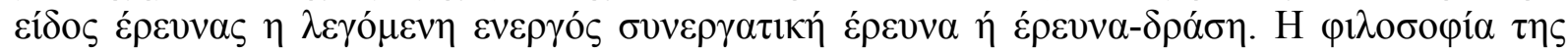

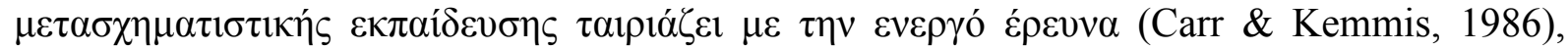

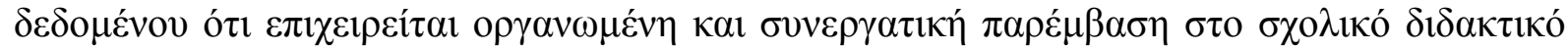

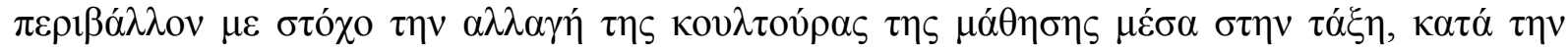

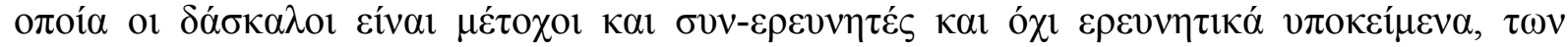

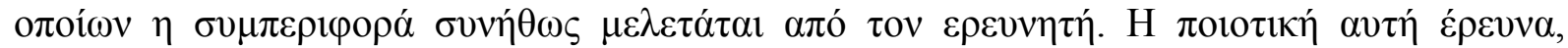

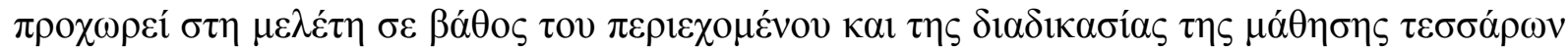

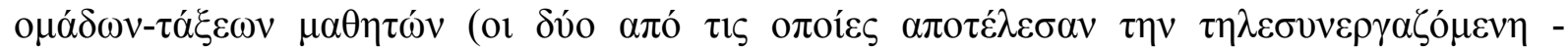

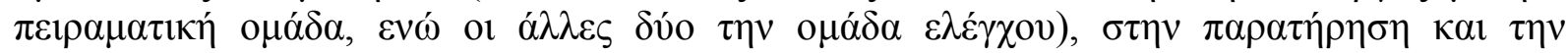

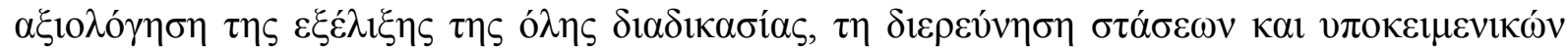

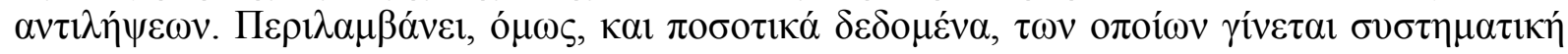

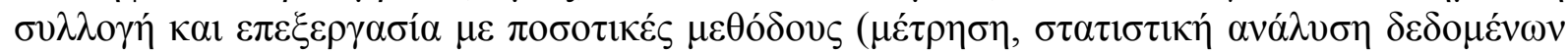

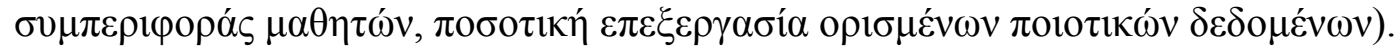

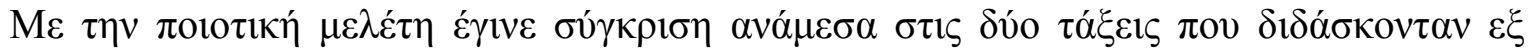

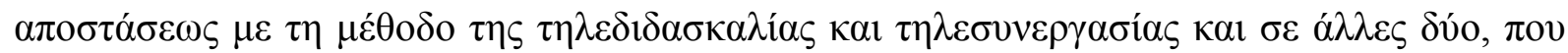

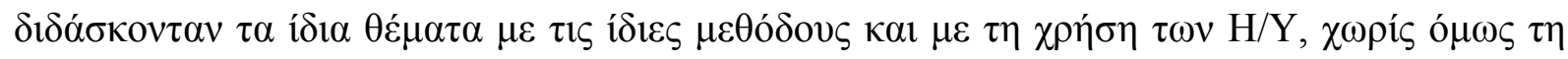

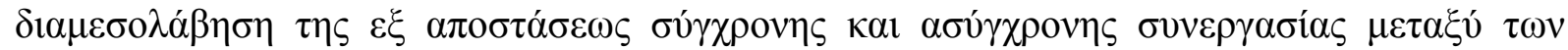

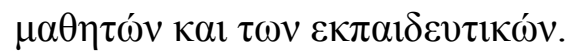

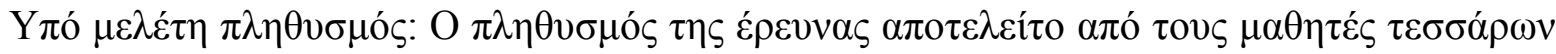

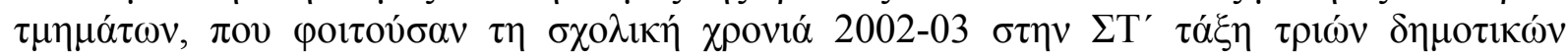

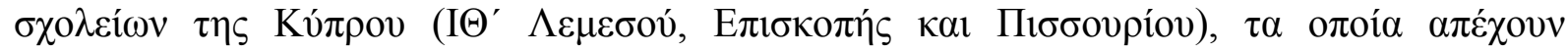

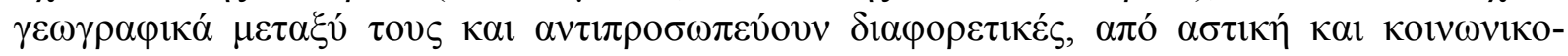

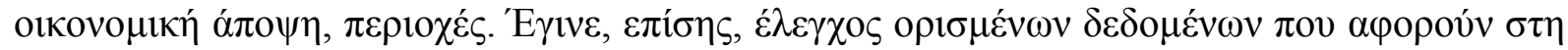

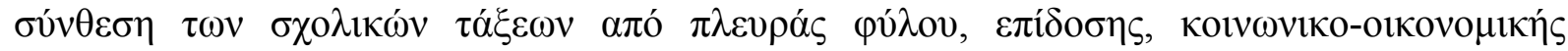

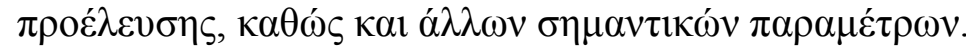

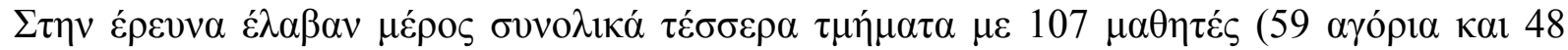

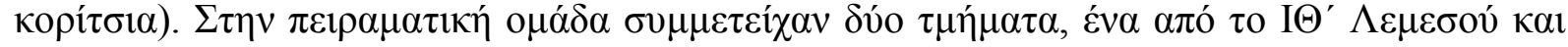

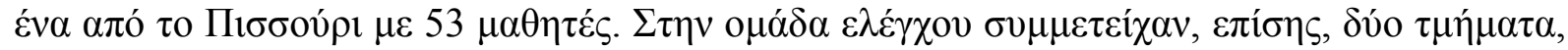

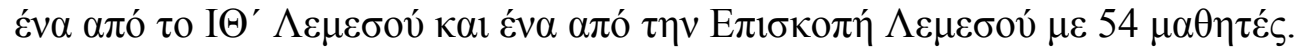




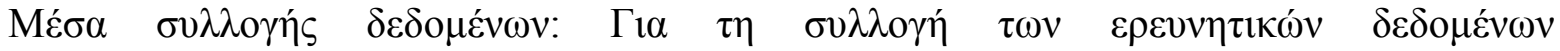

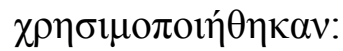

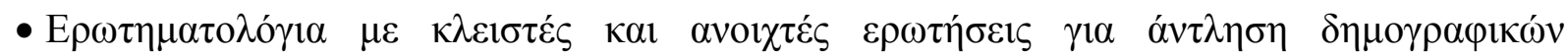

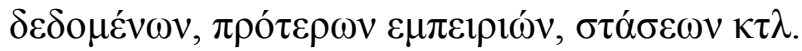

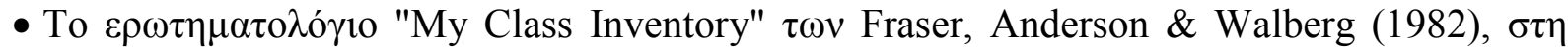

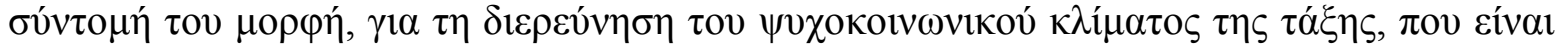

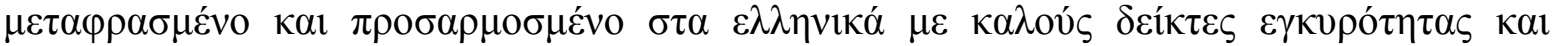

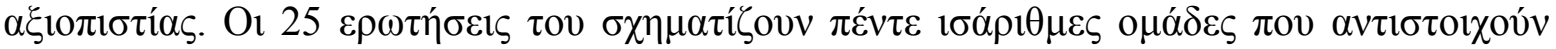

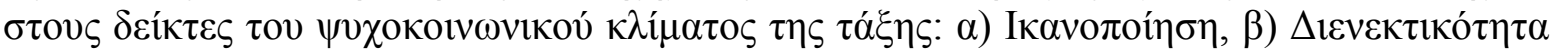

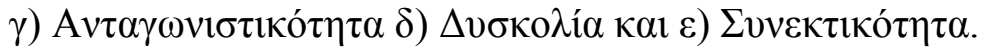

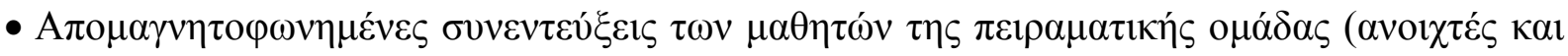
$\eta \mu t-\delta o \mu \eta \mu \varepsilon ́ v \varepsilon \varsigma)$.

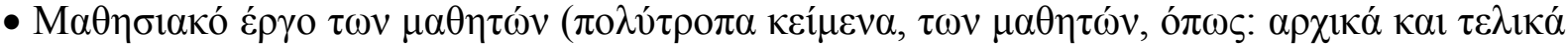

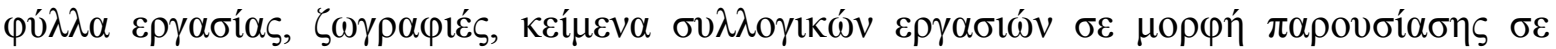

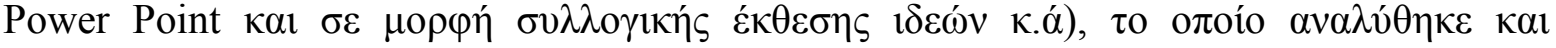

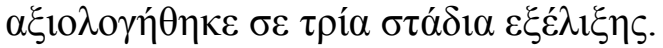

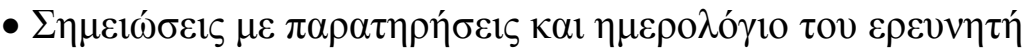

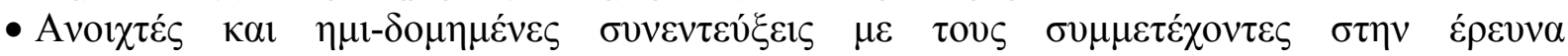

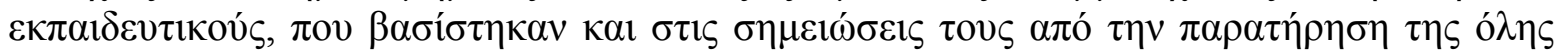

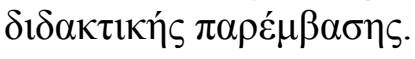

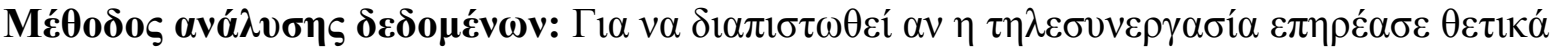

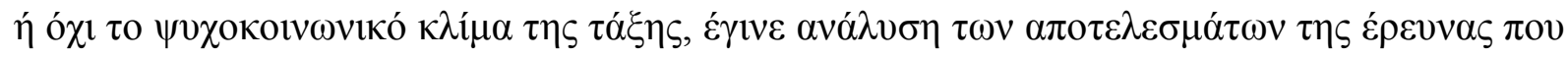

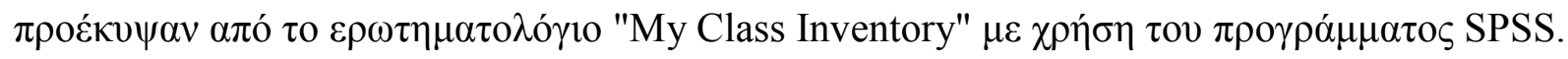

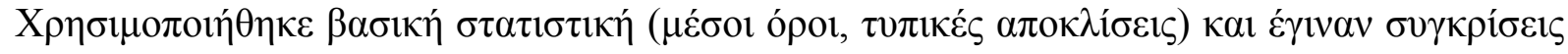

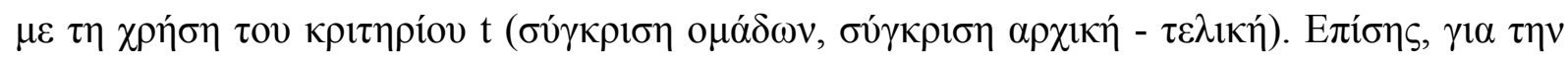

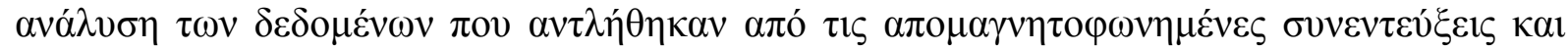

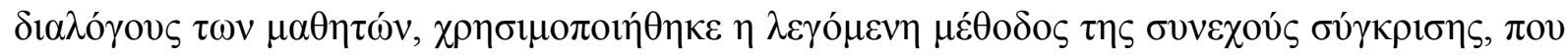

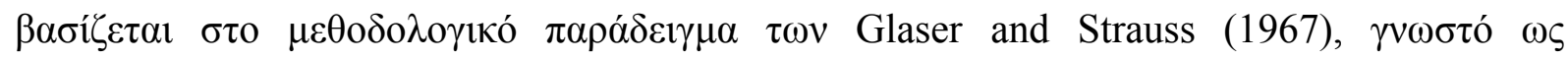

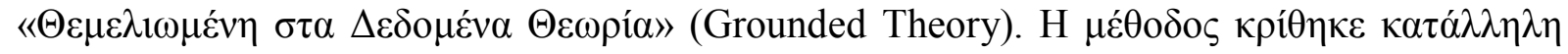

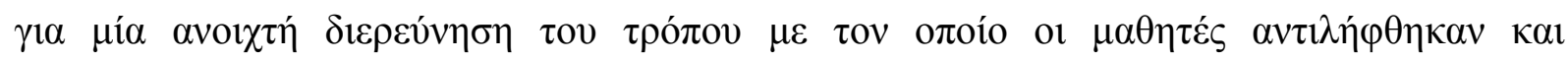

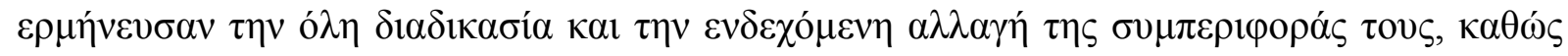

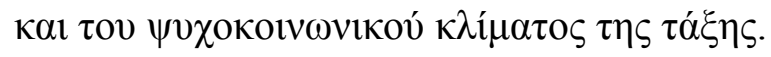

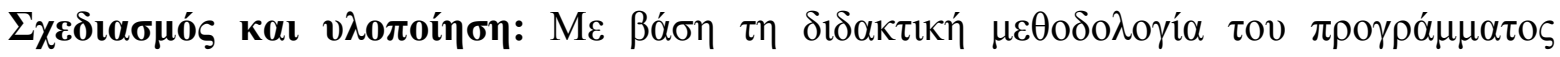

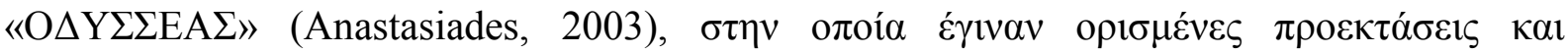

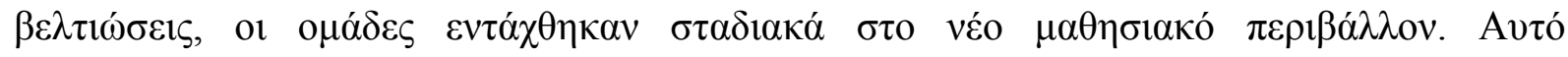

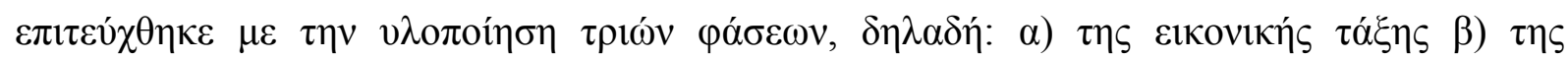

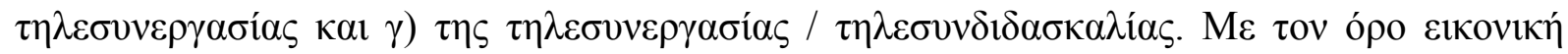

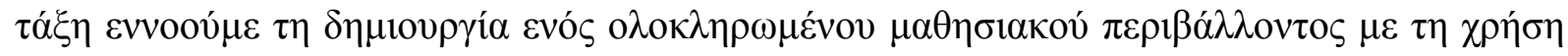

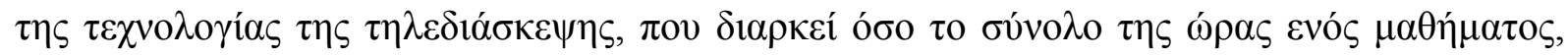

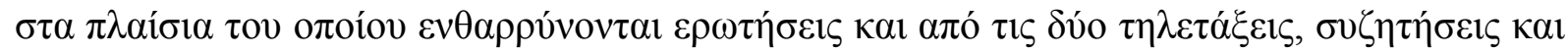

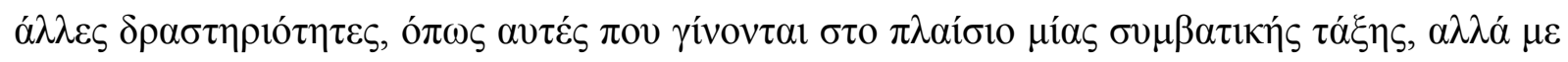

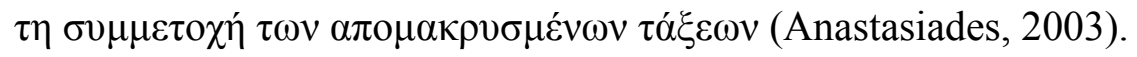




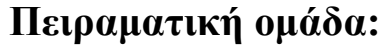

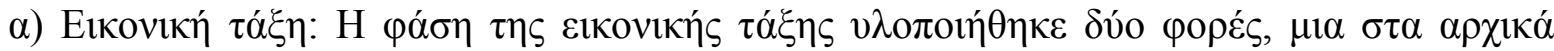

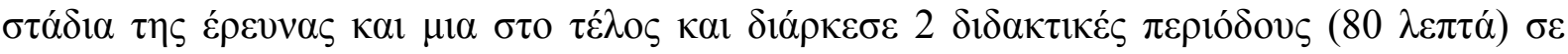

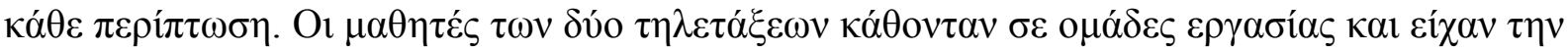

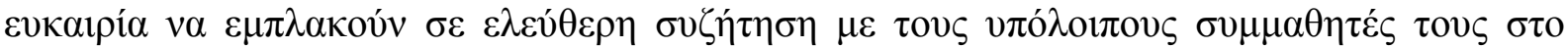

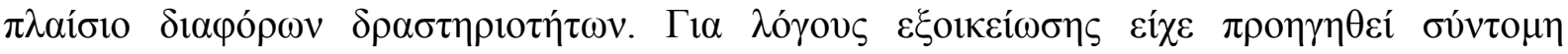

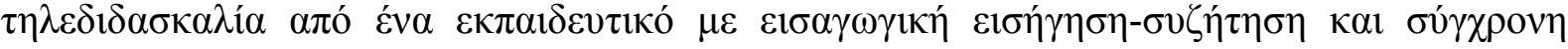

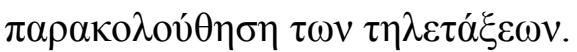

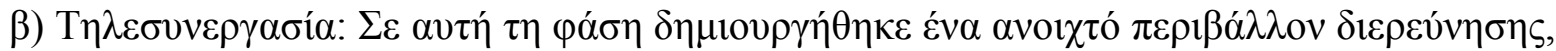

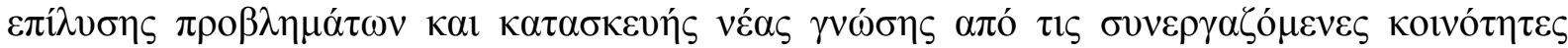

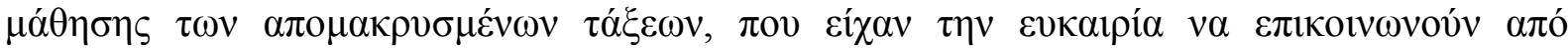

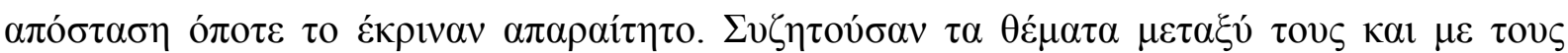

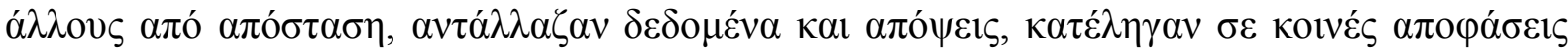

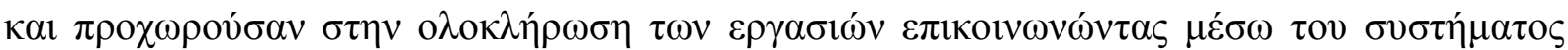

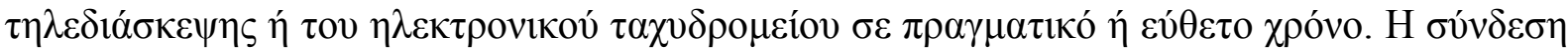

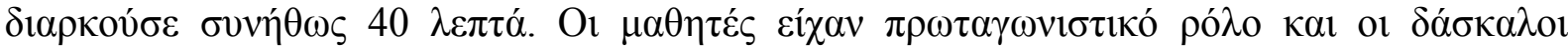

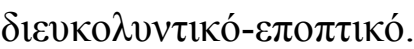

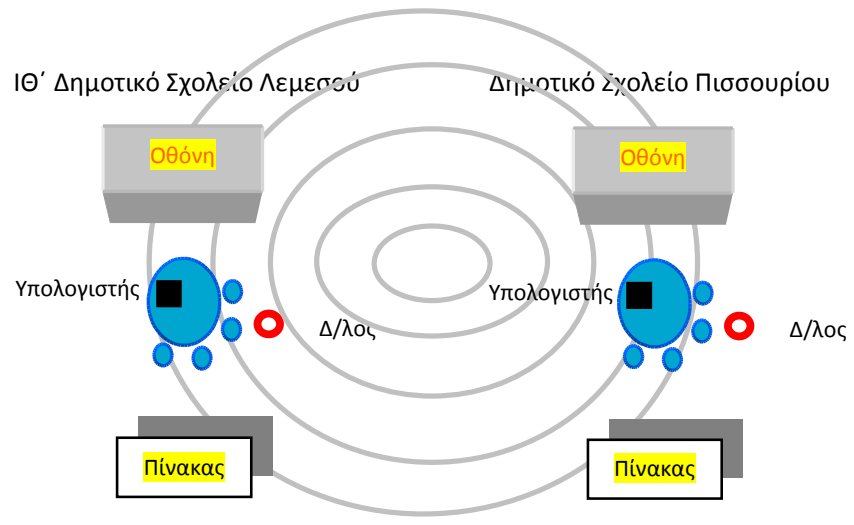

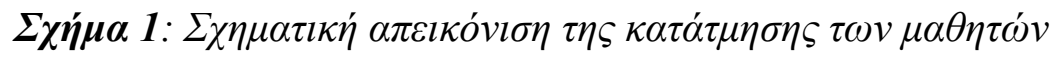




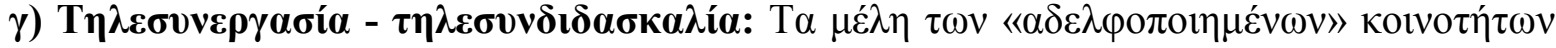

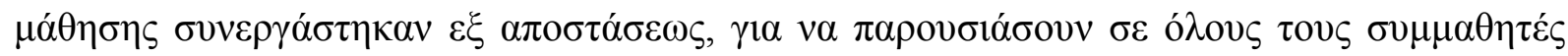

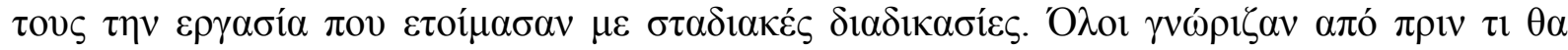

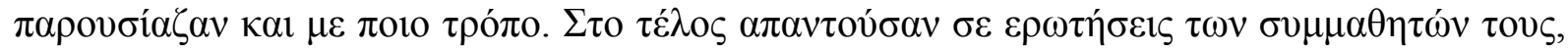

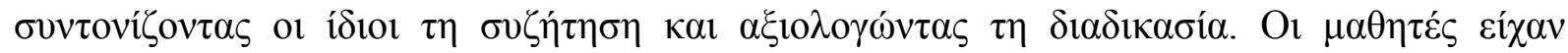

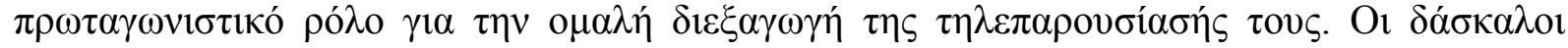

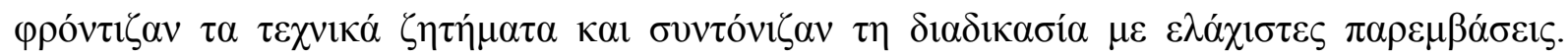

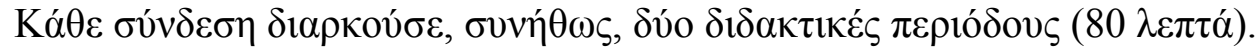

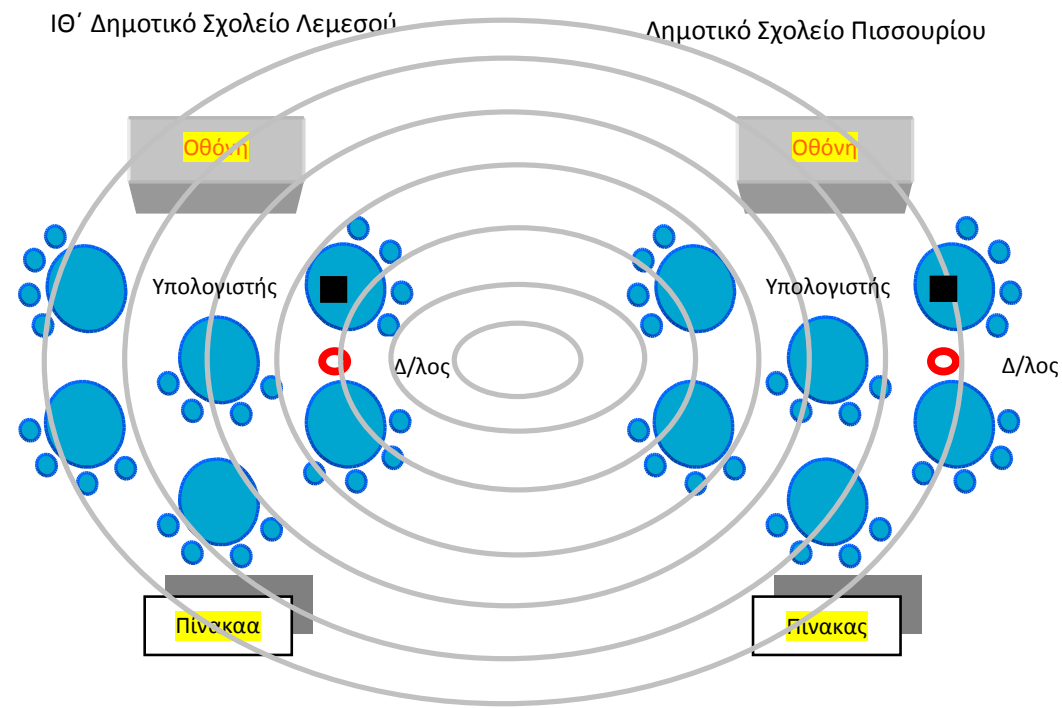

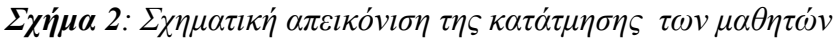
$\sigma \tau \iota \varsigma \tau \alpha^{\prime} \varepsilon l \subset$

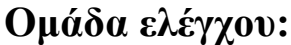

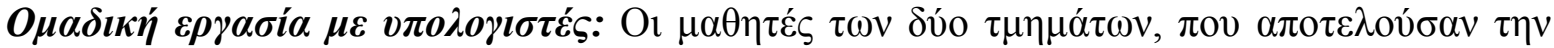

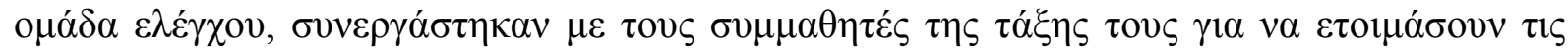

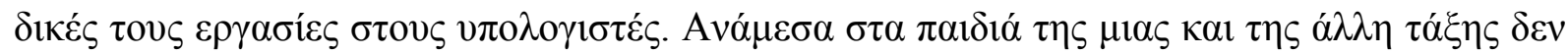

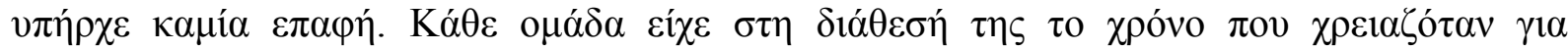

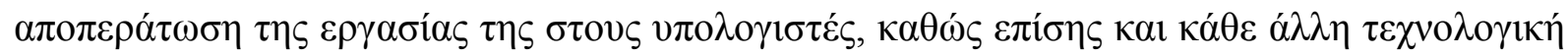

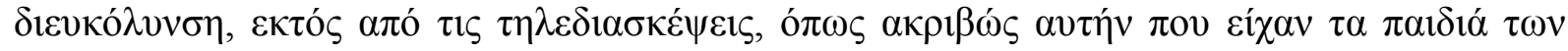

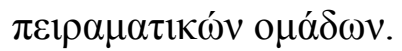

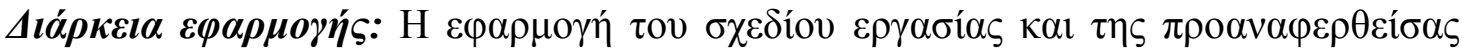

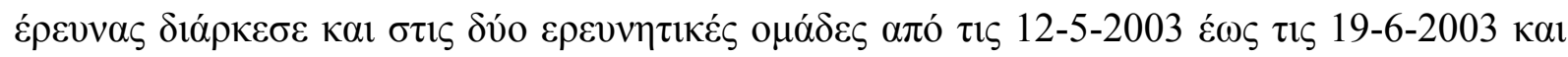
$\pi \varepsilon \rho \imath \lambda \alpha ́ \mu \beta \alpha v \varepsilon$ : 


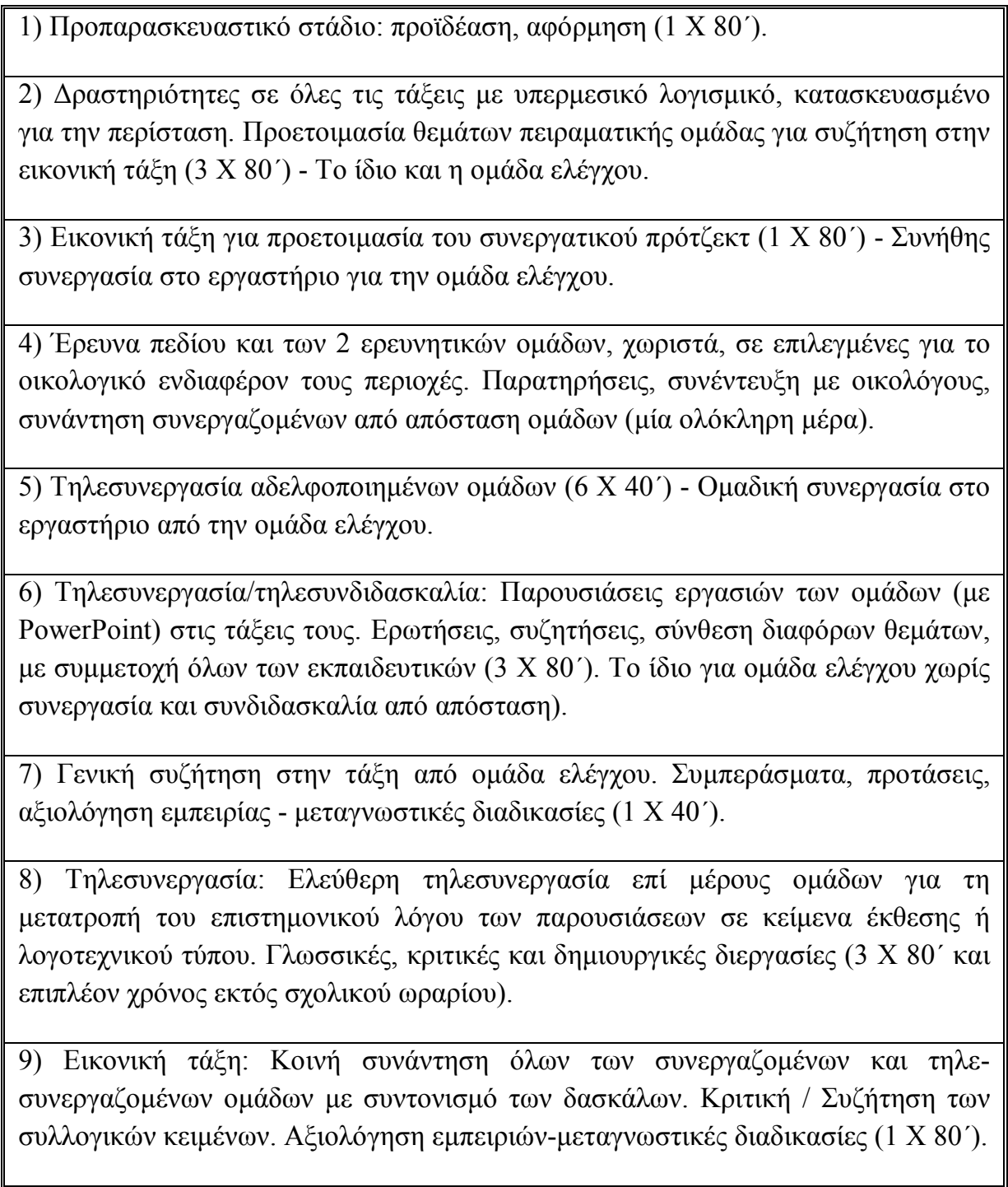

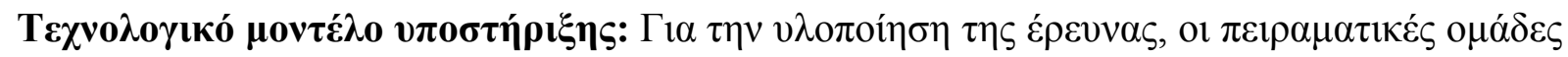

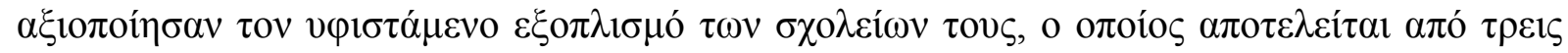

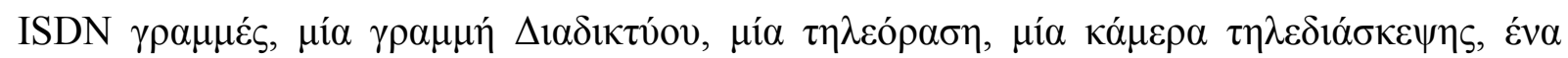

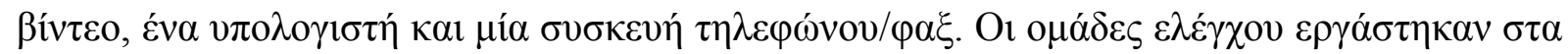

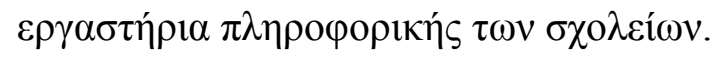

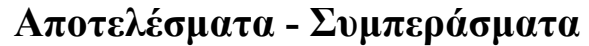

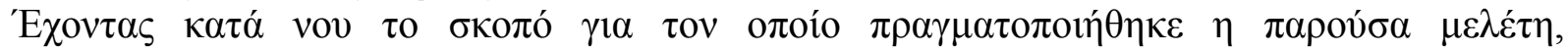

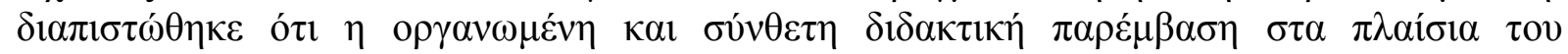

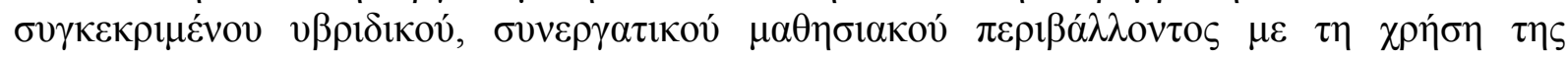

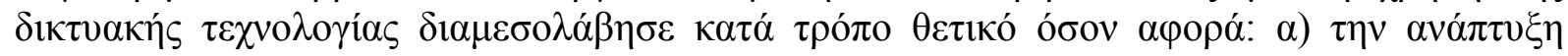

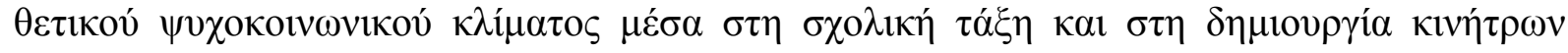

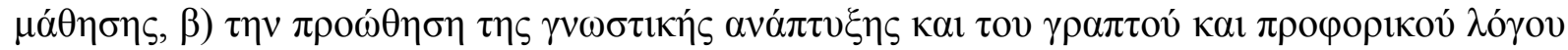

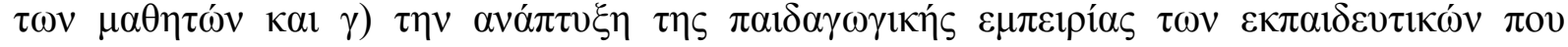




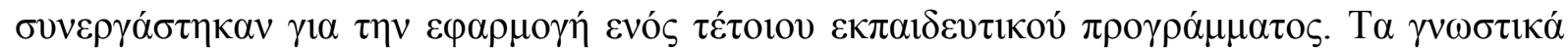

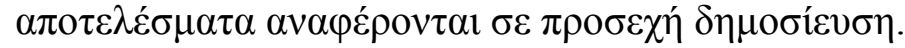

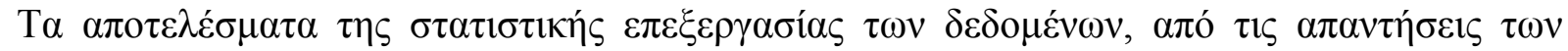

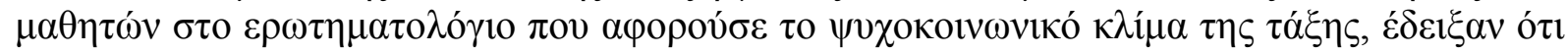

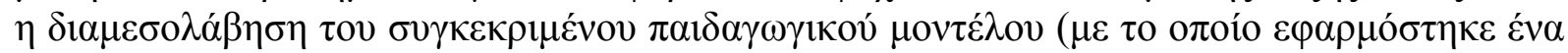

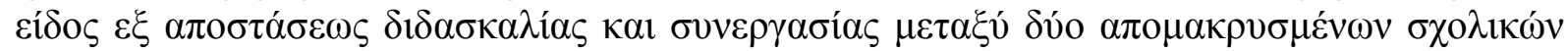

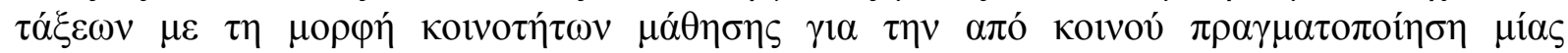

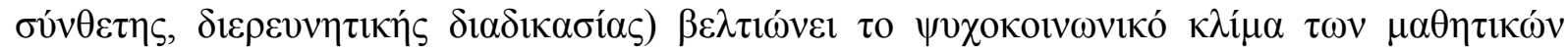

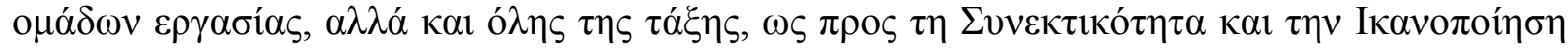

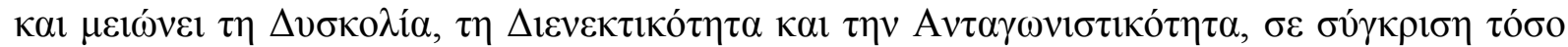

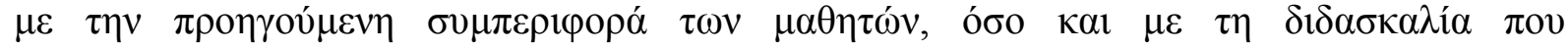

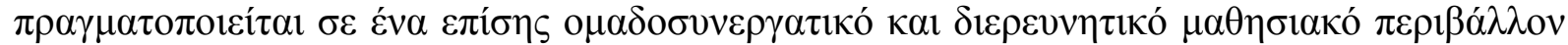

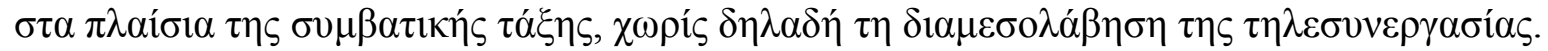

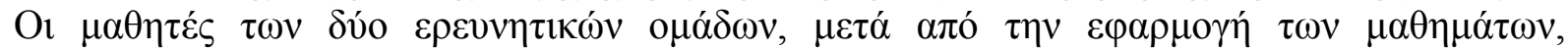

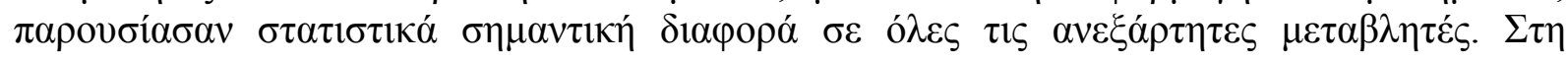

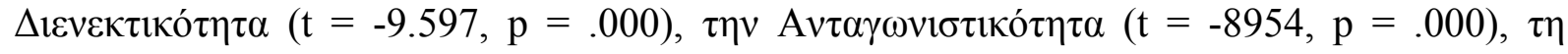

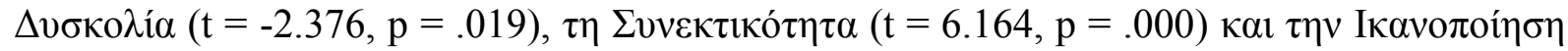
$(\mathrm{t}=10.703, \mathrm{p}=.000)$.

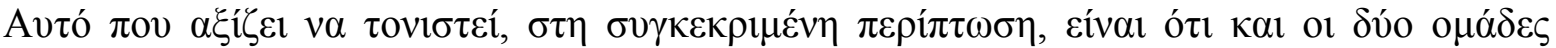

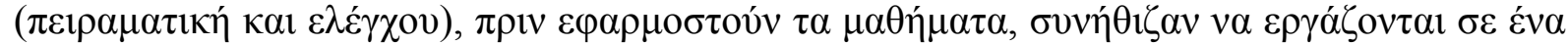

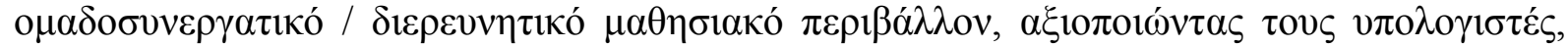

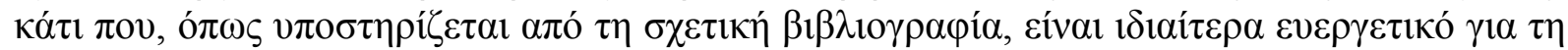

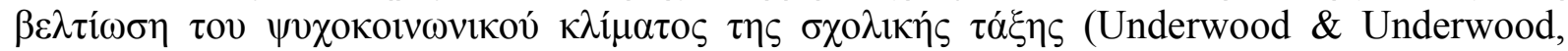

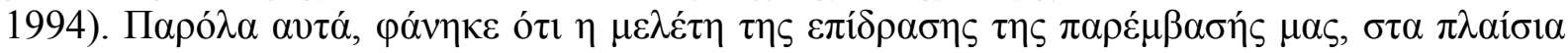

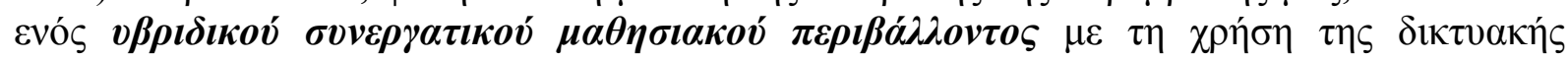

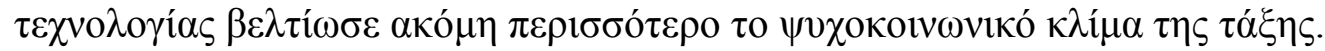

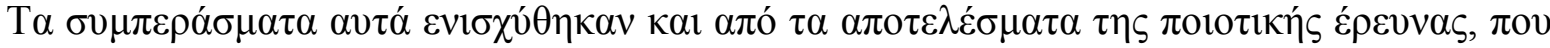

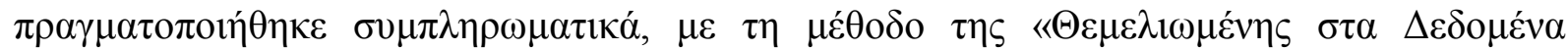

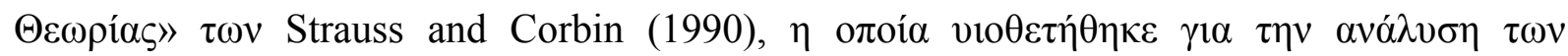

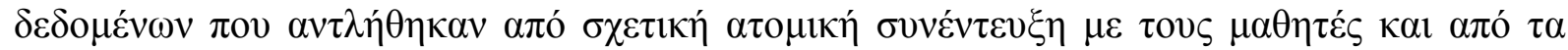

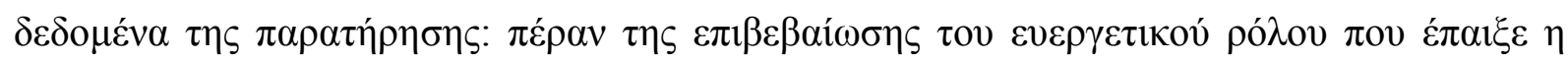

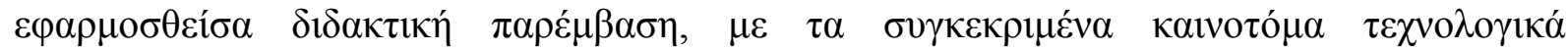

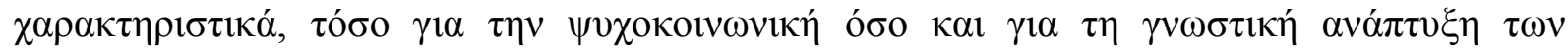

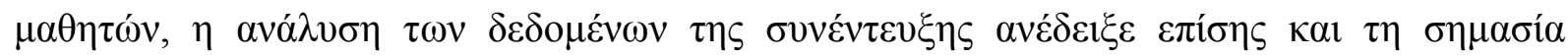

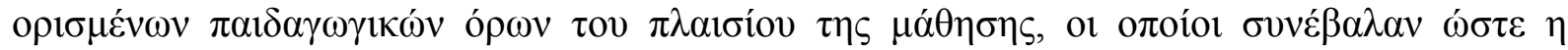

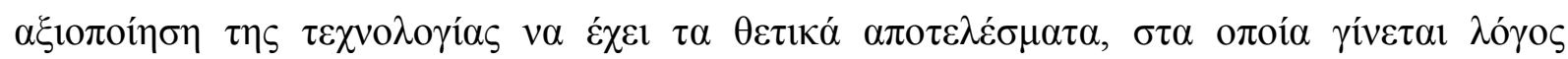
$\pi \alpha \rho \alpha \kappa \alpha ́ \tau \omega$.

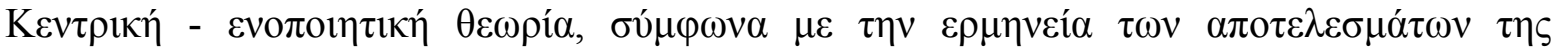

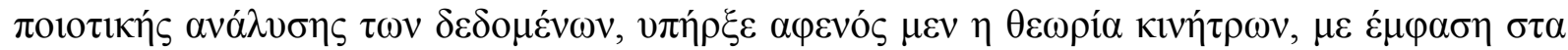

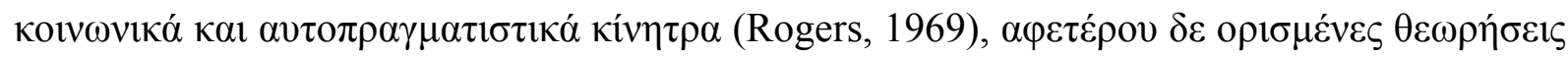

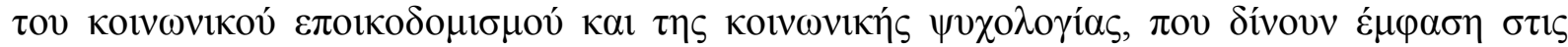

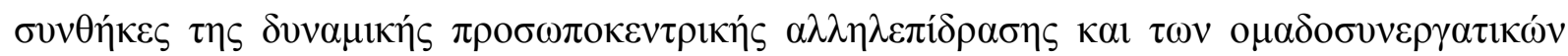

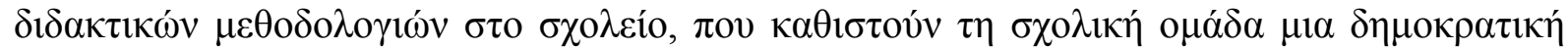

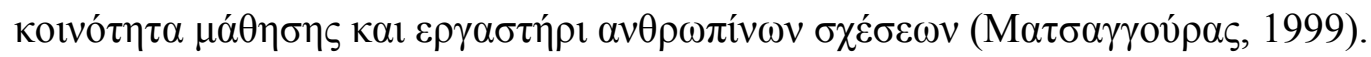




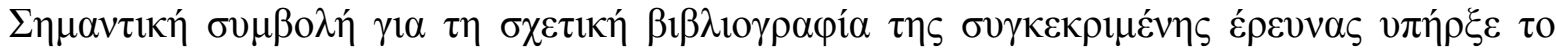

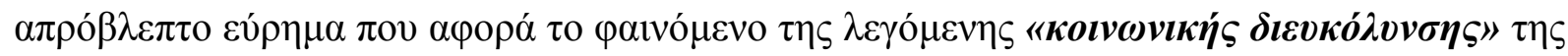

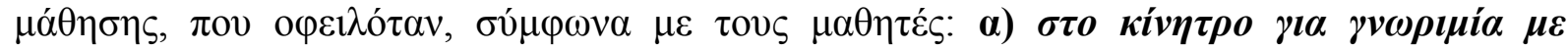

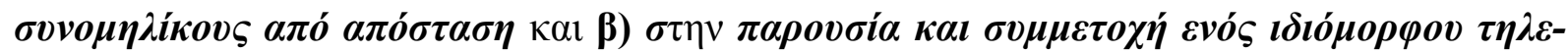

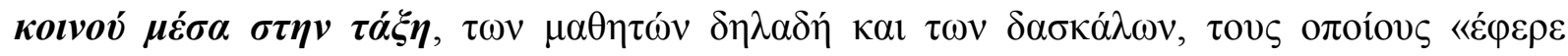

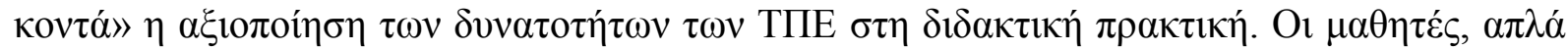

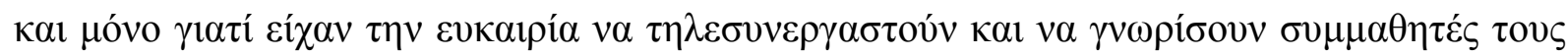

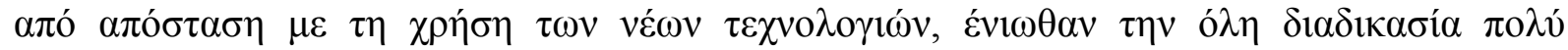

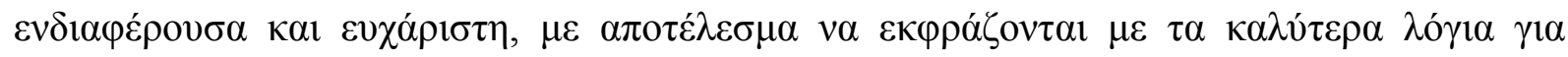

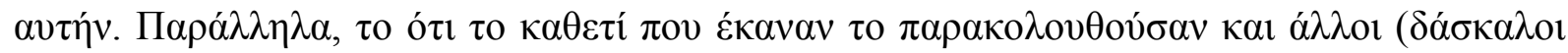

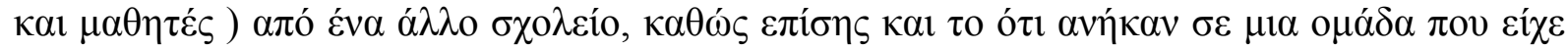

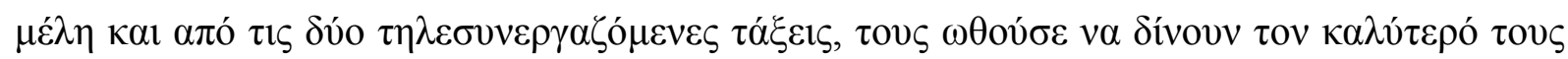

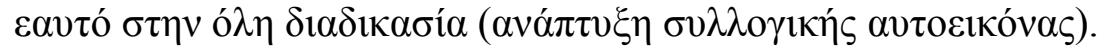

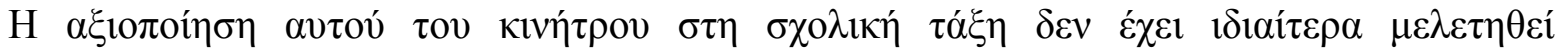

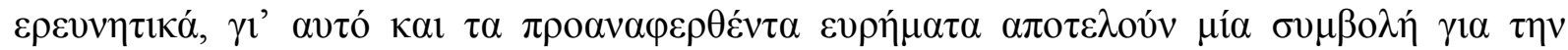

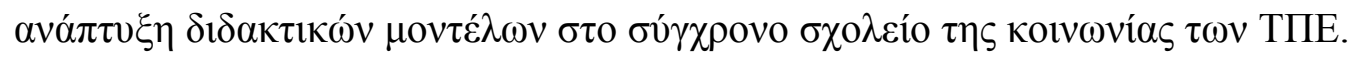

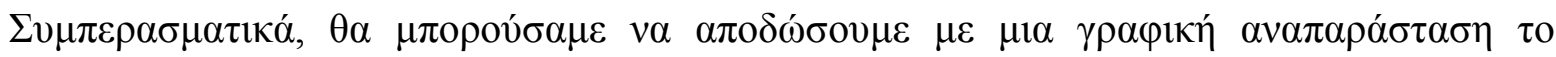

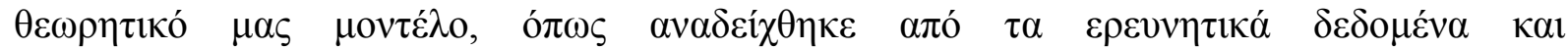

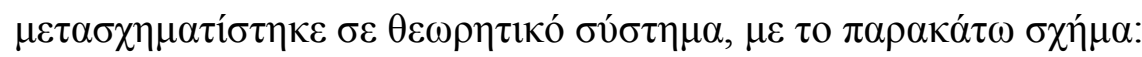

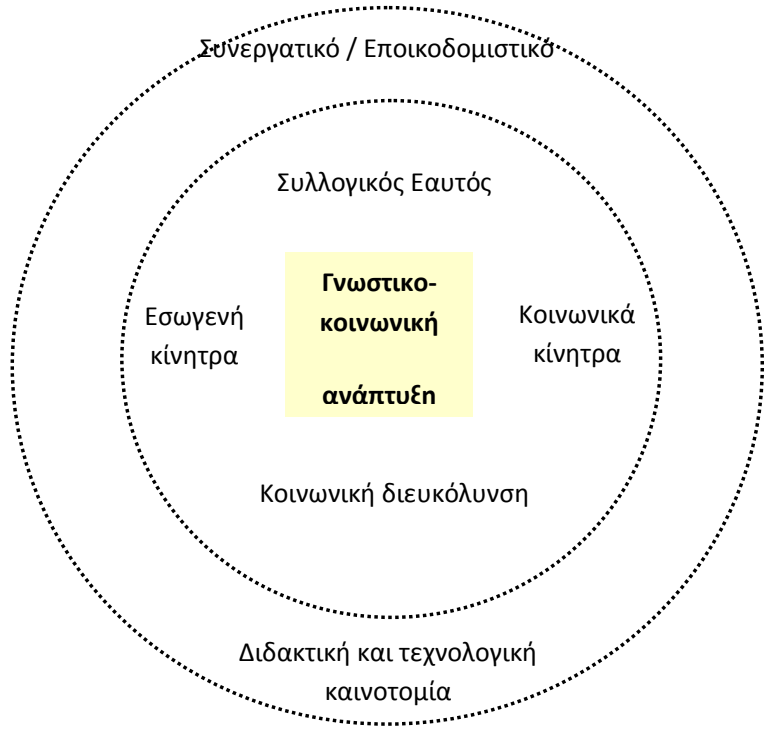




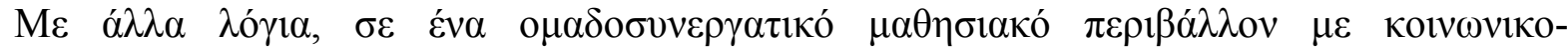

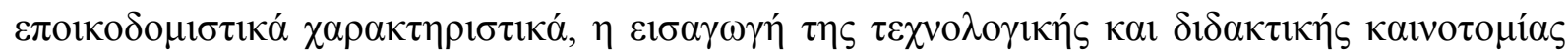

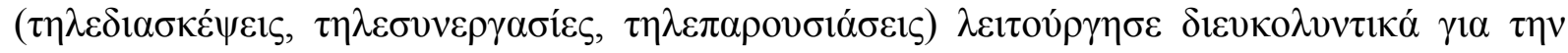

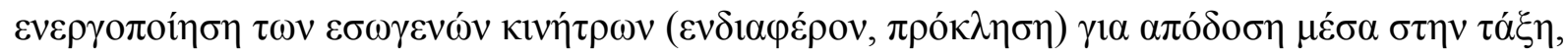

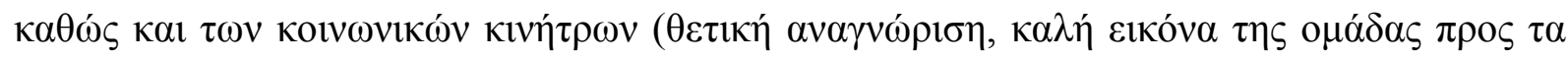

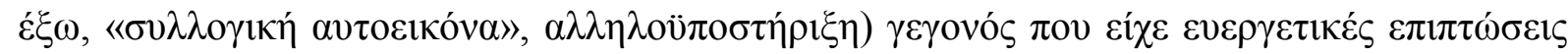

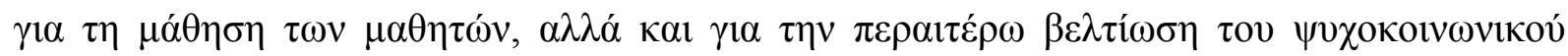

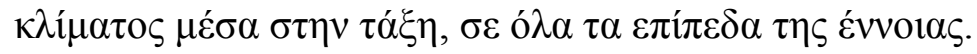

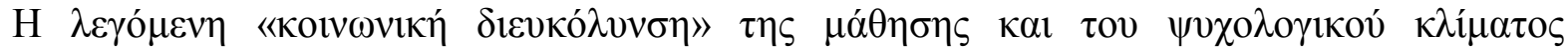

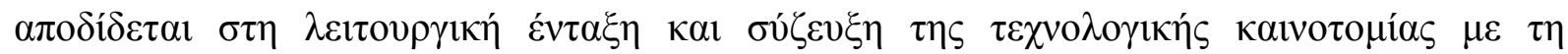

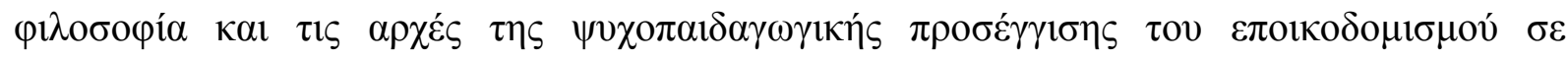

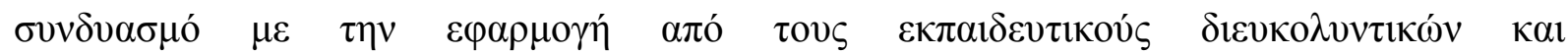

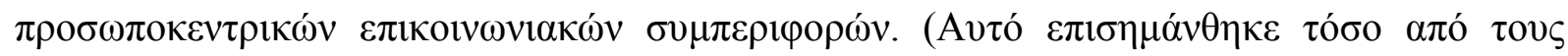

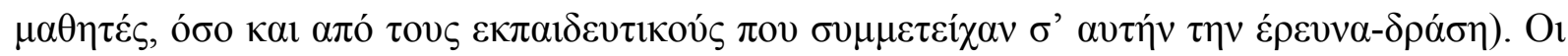

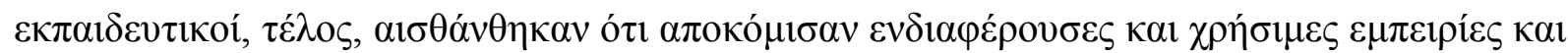

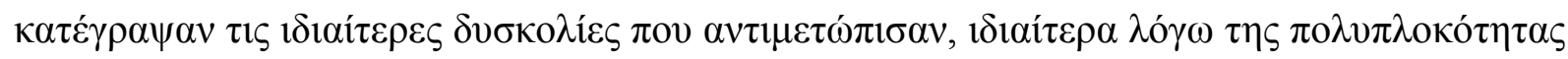

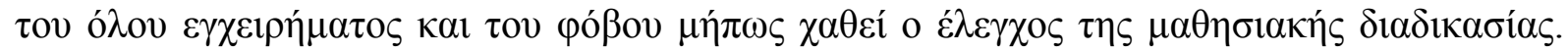

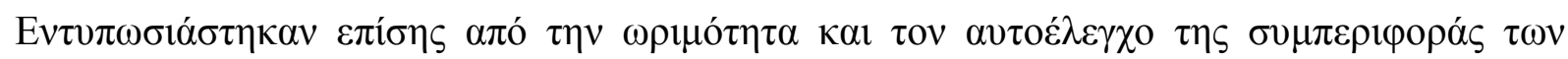

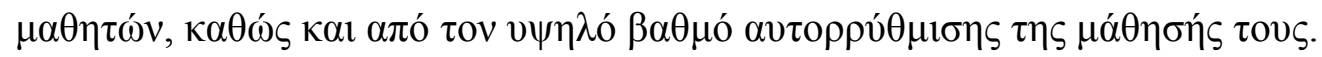

\section{$\Sigma \eta \mu \varepsilon เ \omega ́ \sigma \varepsilon เ \varsigma$}

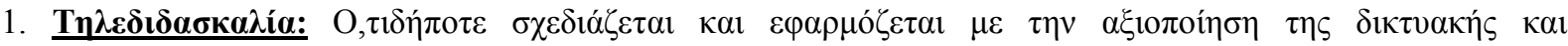

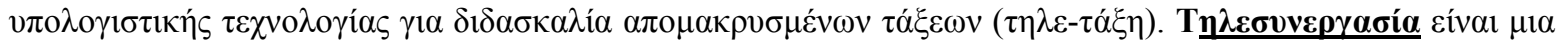

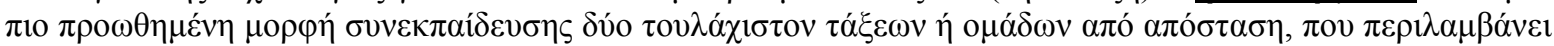

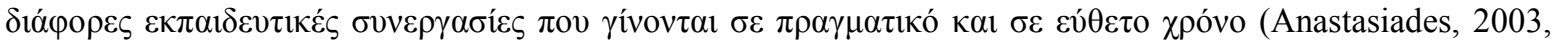

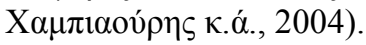

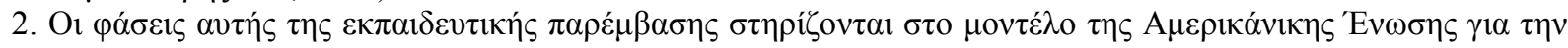

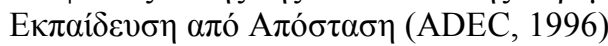

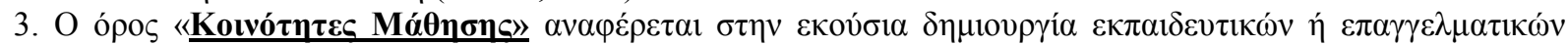

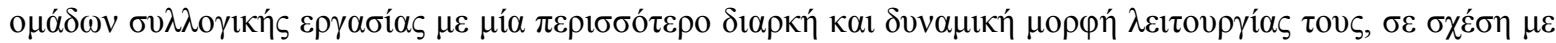

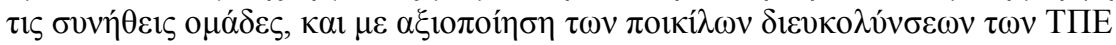

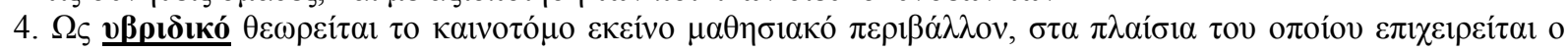

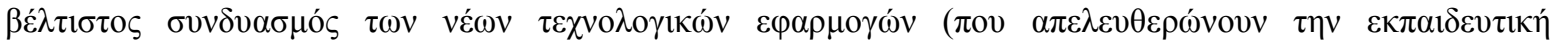

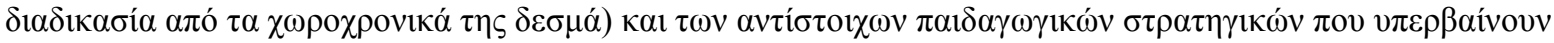

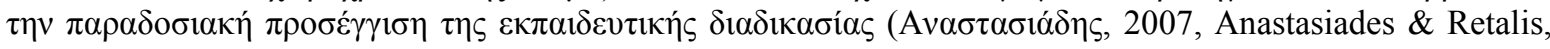
2002,).

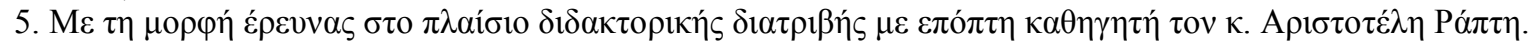

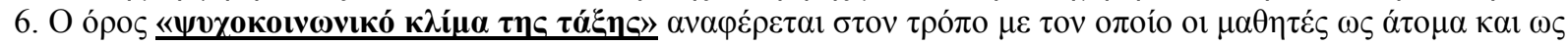

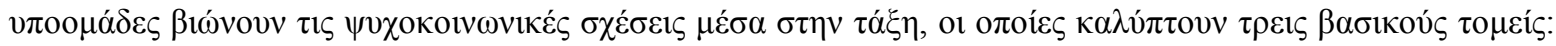

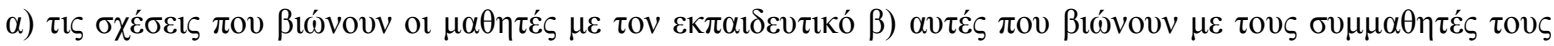

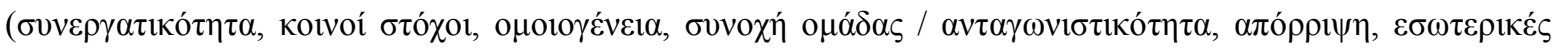

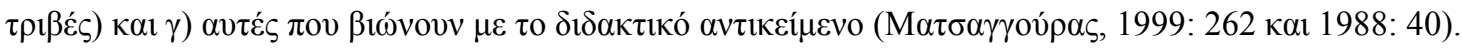

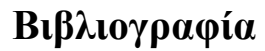




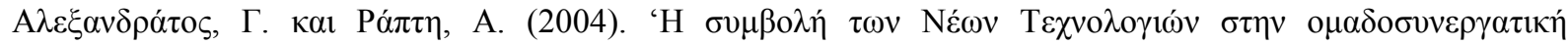

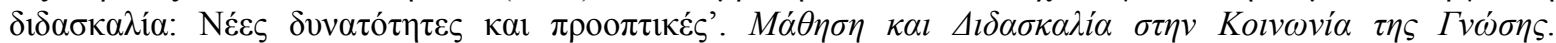

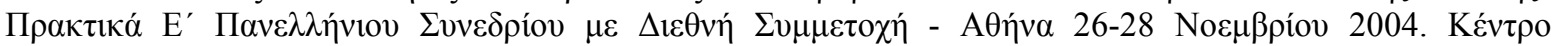

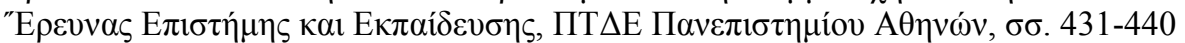

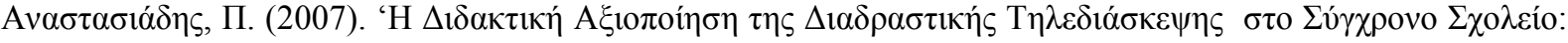

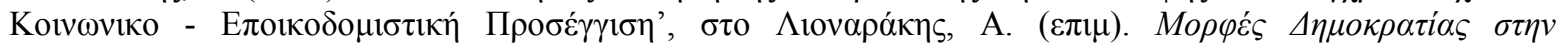

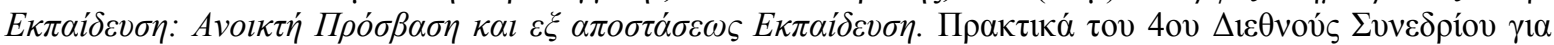

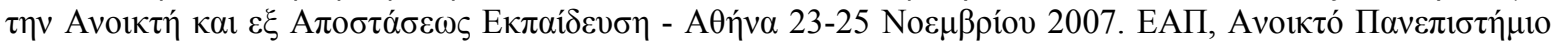

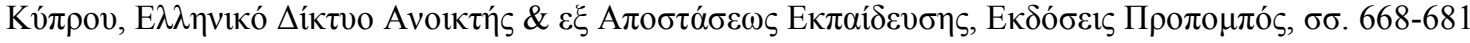

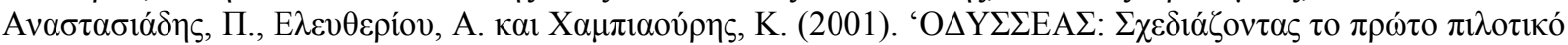

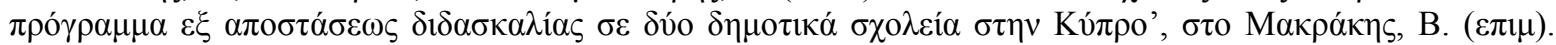

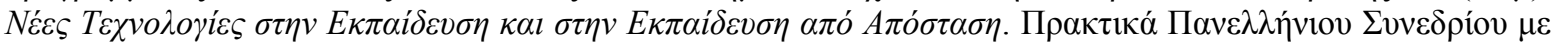

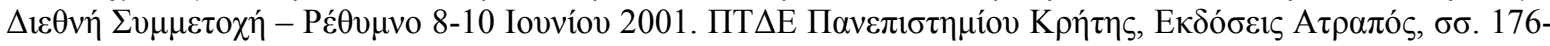
187

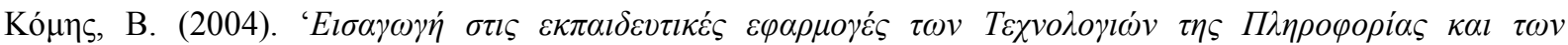

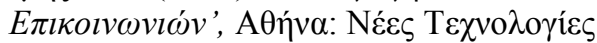

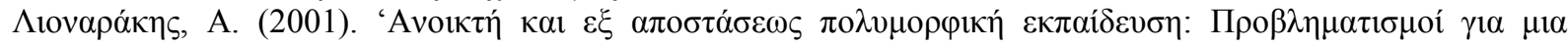

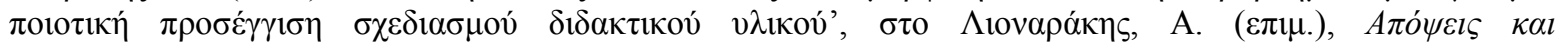

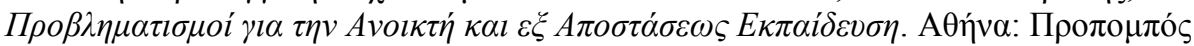

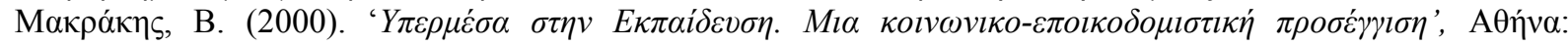

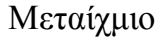

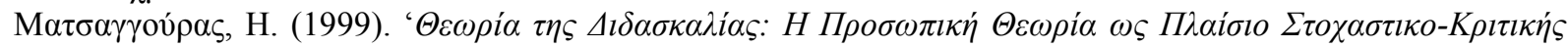

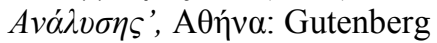

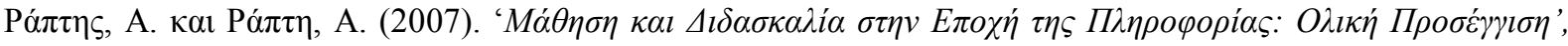
A $\theta$ ñva

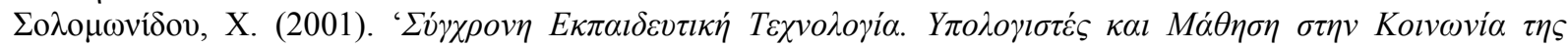

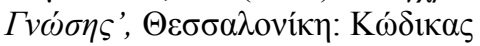

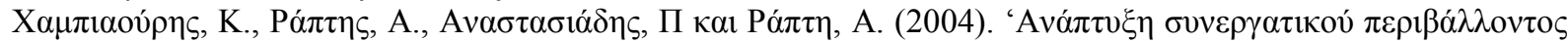

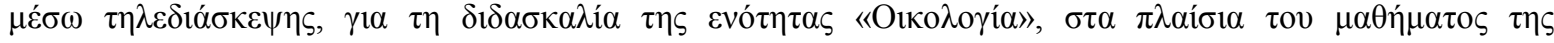

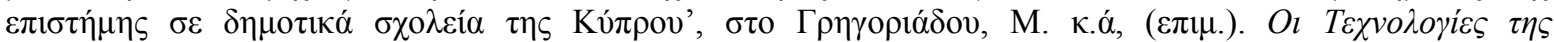

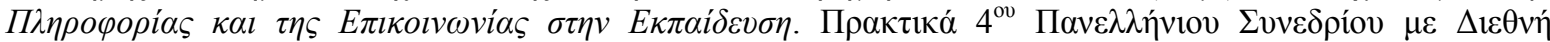

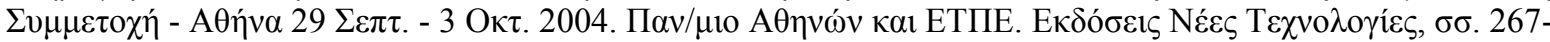
276

American Distance Education Consortium - ADEC (1999). (http://www.adec.edu/admin/papers/distanceteaching_principles.html)

Anastasiades, P. (2003). 'Distance Learning in Elementary Schools in Cyprus: The evaluation Methodology and Results'. Computers \& Education, 40 (1), pp. 17-40

Anastasiades, P. and Retalis, S. (2002). 'The Educational Process in the Emerging Information Society: Conditions for the Reversal of the Linear Model of Education and the Development of an Open Type Hubrid Learning Environment'. Computers in the Social Studies Journal, 10 (1)

Carr, W. and Kemmis, S. (1986). 'Becoming Critical', London: The Palmer Press

Cole, M. (1996). 'Culture in mind', Harvard University Press, Cambridge, M.A.

Dillenbourg, P., Baker, M., Blaye, A. and O’Malley, C. (1995). 'The evolution of research on collaborative learning', in P. Reismann and H. Spada (eds): Learning in Humans and Machines: Towards an Interdisciplinary Learning Science. Elsevier Science Ltd., Oxford New York, Tokyo

Duffy, T., Lowyck, J. and Jonassen, D. (Eds) (1993). 'The design of constructivistic learning environments: Implications for instructional design and the use of technology', Heidelburg, FRG: Springer-Verlag

Foucault, M. (1969/1972). 'The archaeology of knowledge', Random House, N.Y.

Fraser, B., Anderson, D. and Walberg, H. (1982). 'Assessment of Learning Environments', Australia: WAIT

Freire, P. (1985). 'Education for Critical Consciousness', Seabury, New York

Glaser, B. and Strauss, A. (1967). 'The discovery of grounded theory: Strategies for qualitative research', Chicago: Aldine

Harley, D. (2001). 'Distance Learning Technologies: Issues, Trends, and Opportunities'. Rehabilitation Education, 15 (1), pp. 111-113

Jonassen, D. and Land, S. (Eds) (2000). 'Theoretical Foundations of Learning Environments', Lawrence Earlbaum Associates

Keegan, D. (1996). 'Foundation of distance education', London and NY: Routledge

Lave, J. and Wenger, E. (1991). 'Situated learning: Legitimate peripheral participation', Cambridge, UK: Cambridge University Press

Picciano, A. (2001). 'Distance learning', Ohio, Merrill Prentice Hall 
Rogers, C. (1969). 'Freedom to Learn for the '80's', Charles Merrill Publishing Company, Columbus, Toronto, London, Sydney

Rosbottom, J. (2001). 'Hybrid learning - a safe route into web-based open and distance learning for the Computer Science teacher'. Sigcse Bulletin, 33 (3), pp. 89-92

Strauss, A. and Corbin, J. (1990). 'Basics of qualitative research: Grounded theory procedures and techniques', Newbury Park, CA: Sage

Underwood, J. and Underwood, G. (1994). 'Computers and Learning', Oxford: Black-well

Vygotsky, L. (1978). 'Mind in society: The development of higher psychological processes', Harvard University Press

Wilson, B. (1996). 'Designing constructivist learning environments', Englewood Cliffs, NJ: Educational Technology Publications 


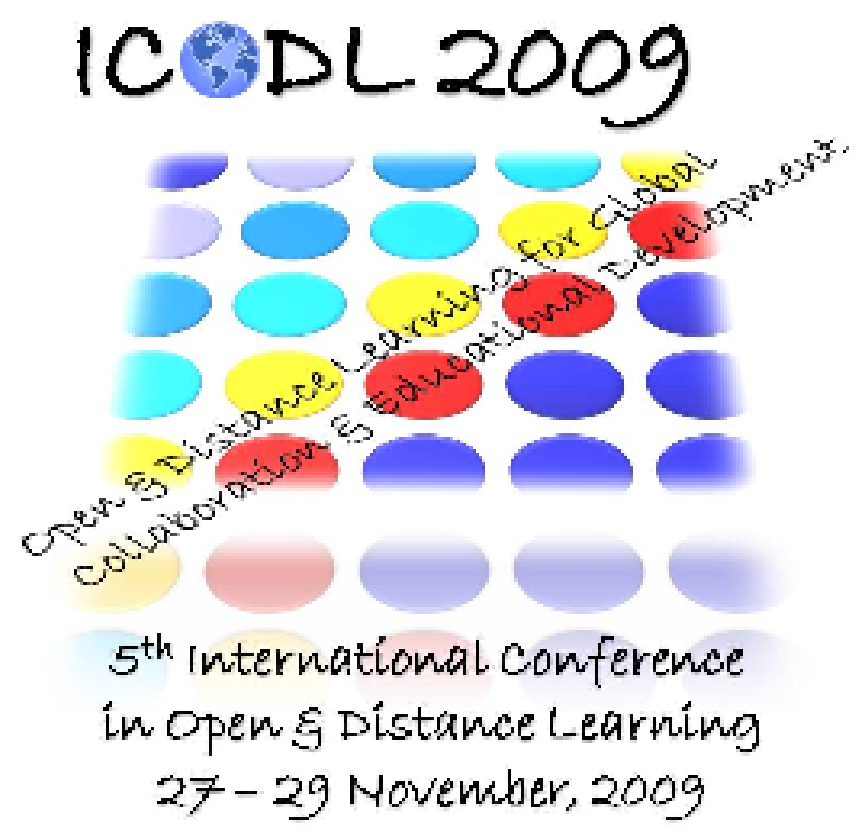

\section{ICODL 2009 \\ Open \& Distance Learning for Global Collaboration and Educational Development}

\section{$5^{\text {th }}$ International Conference in Open \& Distance Learning 27 - 29 November 2009, Athens, Greece}

\section{Organized by}

- The Hellenic Open University

- The Hellenic Network of Open \& Distance Education

- The OPEN EDUCATION International Journal (The Journal for Open and Distance Education and Educational Technology)

\section{IMPORTANT DATES}

\begin{tabular}{|ll|}
\hline Paper submission deadline & May, 31 \\
\hline Notification of Authors & June, 15 \\
\hline Submission of Final Papers & July, 15 \\
\hline Registration & September, 5 \\
\hline & \\
\hline Conference & November 27 -29, 2009 \\
\hline
\end{tabular}




\section{TOPICS:}

- Applications and Research in Open and Distance Education

- Innovations, new and Alternative Forms of Educational Applications in Conventional and Distance Institutions

- New Technology Applications in Communication, Teaching and Learning

- Conventional Teaching Material in Alternative Digital forms: E-learning, M-learning, D-learning, Online learning

- Open Access and Open Education

- Distance and Conventional Education Institutions: Autonomous and Mixed Models for Teaching and Learning

- National and Global Collaboration in Higher Education for Sustainable Development

- European Collaboration of Educational Institutions for Undergraduate and Postgraduate Study Programmes

- Development, Evaluation and Quality Assurance of Higher Education

- International and Cross - Border Higher Education Study Programmes: Thoughts for Development

- Models of Collaboration in Distance Learning for Primary and Secondary Education

- Lifelong and Continuing Education: Collaboration, Possibilities, Perspectives

\section{Information: http://artemis.eap.gr/ICODL2009/}

Past Conferences

$2001 / 1^{\text {st }}$ Conference, 600 participants, 1253 pages of proceedings

$2003 / 2^{\text {nd }}$ Conference, 500 participants, 767 pages of proceedings

$2005 / 3^{\text {rd }}$ Conference, 500 participants, 1278 pages of proceedings

$2007 / 4^{\text {th }}$ Conference, 450 participants, 1337 pages of proceedings 